\title{
Precursor Mediated Microcanonical Trapping Model of Activated Dissociative Chemisorption at Surfaces: Assessment of Dynamical Effects
}

\author{
Scott Bradshaw Donald \\ Lexington, Virginia \\ B.S., Virginia Military Institute, 2006 \\ Doctor of Philosophy \\ Department of Chemistry \\ University of Virginia \\ August 2013
}

A Dissertation presented to the Graduate Faculty of the University of Virginia in Candidacy for the Degree of 
Abstract

In an effort to help bridge the "nonequilibrium gap" between the fields of surface science and heterogeneous catalysis, a precursor mediated microcanonical trapping (PMMT) model developed and applied to several benchmark surface reactions of catalytic interest: $i$ ) the dissociative chemisorption and recombinative desorption of hydrogen on $\mathrm{Cu}(111)$, ii) the dissociative chemisorption of methane on $\operatorname{Pt}(111), \mathrm{Ru}(0001), \mathrm{Ni}(100), \mathrm{Ni}(111)$, and $\operatorname{Ir}(111)$, and iii) the dissociation of several small alkanes on $\mathrm{Pt}(111)$. Currently, the PMMT appears unique in being able to quantitatively compare disparate thermal equilibrium and nonequilibrium experiments concerning activated dissociative chemisorption at surfaces and to connect these experiments to transition state characteristics derived from electronic structure theory (EST) calculations. This thesis work introduced dynamical biases, tunneling, and transition state frequencies derived from EST calculations into PMMT modeling for the first time.

The effects of dynamics, surface temperature, and tunneling on the dissociative chemisorption of hydrogen on $\mathrm{Cu}(111)$ was explored using a dynamically-biased precursor mediated microcanonical trapping (d-PMMT) model. The d-PMMT model reproduces a diverse variety of dissociative chemisorption and associative desorption experimental results, and, importantly, largely captures the surface temperature dependence of quantum-state-resolved dissociative sticking coefficients. The efficacy of molecular rotational energy to promote dissociation, relative to normal translational energy, varied monotonically from $-45 \%$ to $33 \%$ as the rotational energy increased. The molecular vibrational and surface phonon efficacies were $60 \%$. Efficacies did not vary with isotope change from $\mathrm{H}_{2}$ to $\mathrm{D}_{2}$. Dynamical effects are significant and suppress $S(T)$ by $\sim 2$ orders of magnitude as compared to statistical expectations. For thermal dissociative chemisorption of $\mathrm{H}_{2} / \mathrm{Cu}(111)$ at $1000 \mathrm{~K}$, a temperature of catalytic interest, normal translational energy is calculated to provide $74 \%$ of the energy necessary to react, surface phonons $17 \%$, molecular rotation $5 \%$, and 
vibration $4 \%$. Tunneling is calculated to account for $13 \%$ of $S(T)$ at $1000 \mathrm{~K}$, but more than $50 \%$ at temperatures below $400 \mathrm{~K}$.

The reactivity of $\mathrm{CH}_{4}$ impinging on $\mathrm{Pt}(111), \mathrm{Ru}(0001), \mathrm{Ni}(111), \mathrm{Ni}(100)$, and $\operatorname{Ir}(111)$ surfaces was examined using a precursor-mediated microcanonical trapping model of dissociative chemisorption wherein the effects of rotational and vibrational energy could be explored. Dissociative sticking coefficients for a diverse range of non-equilibrium effusive beam, supersonic beam, and eigenstate-resolved experiments were simulated by treating molecular rotations and translation parallel to the surface as spectator degrees of freedom, and introducing a dynamically-biased vibrational efficacy. The efficacy of vibration was found to vary from 0.05 for $\operatorname{Ir}(111)$ to 0.72 for $\mathrm{Ni}(100)$. At catalytically relevant temperatures, surface phonons were found to play an important role in promoting reactivity on all surfaces. Relationships between the transition state properties calculated using electronic structure theory and experimentally derived d-PMMT parameters were explored.

A precursor mediated microcanonical trapping model of activated dissociative chemisorption was used to analyze effusive molecular beam measurements of dissociative sticking coefficients, $S_{n}\left(T_{g}, T_{s}\right)$, for light alkanes incident along the direction of the surface normal to Pt(111). Explicit accounting of the gas-surface energy transfer for non-equilibrium experiments became increasingly important as the alkane size was increased. A simple exponential down model of the molecule/phonon collision step size distribution with a mean energy down parameter of $\alpha=700$ $\mathrm{cm}^{-1}$ for ethane and $1400 \mathrm{~cm}^{-1}$ for propane sufficed to provide a good description of the $S_{n}\left(T_{g}, T_{s}\right)$ data. An Evans-Polanyi plot of $E_{a}$ for alkane dissociative chemisorption evaluated at $\mathrm{T}=600 \mathrm{~K}$ versus the alkane thermal desorption energy, $E_{d}$, is linear with a slope of -0.608 . Assuming that the alkane $E_{d}$ serves as a good approximation to the van der Waals stabilization of the chemisorbed products of dissociative chemisorption, the slope of the Evans-Polanyi plot indicates a slightly late 

state transition barrier for alkane dissociative chemisorption on $\operatorname{Pt}(111)$ with respect to van der Waals binding to the surface. 


\section{Table of Contents}

$\begin{array}{ll}\text { 1. } & \text { Research Motivation }\end{array}$

2. RRKM Simulation of Hydrogen Dissociation on $\mathrm{Cu}(111): \quad 6$

Addressing Dynamical Biases, Surface Temperature, and Tunneling

$\begin{array}{ll}\text { 2.1. Introduction } & 7\end{array}$

2.2. d-PMMT Model 11

$\begin{array}{ll}\text { 2.3. Results and Discussion } & 18\end{array}$

2.3.A. Associative Desorption at $925 \mathrm{~K} \quad 19$

2.3.A.I. $\quad$ Rovibrationally-Resolved Translational Energy Distributions 19

2.3.A.II. Rovibrational Product State Distributions 21

2.3.B. Associative Desorption: Angular Distributions 23

2.3.C. Associative Desorption: Surface Temperature Dependence of $P_{\mathrm{v}, \mathrm{J}}\left(E_{t} ; \vartheta=0^{\circ}\right)$

2.3.D. Non-equilibrium Dissociative Sticking: 26

Supersonic Molecular Beams with Surface at $T_{s}=120 \mathrm{~K}$.

2.3.E. Thermal Dissociative Sticking: Ambient Gas 26

2.3.F. Comparison to Other Work 29

2.3.F.I. $\quad$ Analysis with Error Function Sticking Model 29

$\begin{array}{lll}\text { 2.3.F.II. Dynamical Simulations } & 32\end{array}$

$\begin{array}{lll}\text { 2.4. Summary } & 33\end{array}$

$\begin{array}{lr}\text { Figures and Tables } & 34\end{array}$

$\begin{array}{ll}\text { References } & 54\end{array}$

3. $\quad$ RRKM Simulation of Methane Dissociation on $\operatorname{Pt}(111): \quad 58$

Addressing Vibrational Efficacy and Spectator Rotation

$\begin{array}{lll}3.1 & \text { Introduction } & 59\end{array}$

3.2. Precursor Mediated Microcanonical Trapping Theory 62 
$\begin{array}{lll}\text { 3.2.A } & \text { s-PMMT Model } & 62\end{array}$

3.2.B. d-PMMT Model 66

$\begin{array}{llr}\text { 3.2.C. } & \text { Tunneling }\end{array}$

3.3. Results and Discussion $\quad 71$

3.3.A. Vibrational Efficacy $\quad 71$

$\begin{array}{lll}\text { 3.3.B. } & \text { Rotation as a Spectator } & 74\end{array}$

$\begin{array}{lll}\text { 3.3.C. } & \text { Associative Desorption } & 78\end{array}$

3.3.D. Reactivity in Supersonic Beam Experiments $\quad 81$

3.3.E. $\quad$ Effective Activation Energies $\quad 84$

3.3.F. Reactivity Under Thermal Equilibrium Conditions $\quad 89$

3.3.G. $\quad$ Comparison to Other Work 92

3.3.G.1. Quantum/Classical Dynamics 93

3.3.G.2. Empirical Erf Model with Distributions of Dynamical Barriers 94

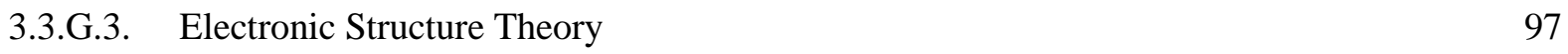

$\begin{array}{llr}\text { 3.3.G.4. Catalysis } & 98\end{array}$

$\begin{array}{ll}\text { 3.4. Summary } & 99\end{array}$

$\begin{array}{ll}\text { Figures and Tables } & 101\end{array}$

$\begin{array}{ll}\text { References } & 127\end{array}$

4. $\quad$ RRKM Simulation of Methane Dissociation on Ru(0001), Ni(111), Ni(100), 132 and Ir(111): Connecting d-PMMT Parameters to Electronic Structure Theory

4.1. Introduction 133

4.1.A. $\quad \mathrm{Ni}(100) \& \mathrm{Ni}(111) \quad 133$

4.1.B. $\quad \operatorname{Ru}(0001) \quad 135$

$\begin{array}{lll}\text { 4.1.C. } & \operatorname{Ir}(111) & 139\end{array}$

4.2. d-PMMT Model 139 
4.3. Results and Discussion - 143

4.3. 143

4.3.A. $\quad \operatorname{Ru}(0001) \quad 143$

$\begin{array}{lll}\text { 4.3.B. } & \mathrm{Ni}(111) & 148\end{array}$

$\begin{array}{ll}\text { 4.3.C. } \mathrm{Ni}(100) & 150\end{array}$

$\begin{array}{lll}\text { 4.3.D. } \operatorname{Ir}(111) & 154\end{array}$

4.3.E. Comparison Between Surfaces $\quad 155$

$\begin{array}{lll}\text { 4.3.E.1. Catalysis } & 155\end{array}$

4.3.F. Polanyi Relationship 158

$\begin{array}{lll}\text { 4.4. Summary } & 159\end{array}$

$\begin{array}{lr}\text { Figures and Tables } & 160\end{array}$

$\begin{array}{ll}\text { References } & 187\end{array}$

5. RRKM Simulation of Small Alkane Dissociation on Pt(111): 193

Addressing Energy Transfer with a Master Equation

$\begin{array}{ll}\text { 5.1. Introduction } & 194\end{array}$

5.2. ME-PMMT Model 196

5.3. Results and Dissussion 203

$\begin{array}{lll}\text { 5.3.A. } & \text { Methane } & 203\end{array}$

$\begin{array}{lll}\text { 5.3.B. Ethane } & 203\end{array}$

$\begin{array}{lll}\text { 5.3.C. Propane } & 204\end{array}$

5.3.D. Angle-Resolved Thermal Dissociative Sticking Coefficients 206

$\begin{array}{lll}\text { 5.3.E. } & \text { Evans-Polanyi Relation } & 207\end{array}$

5.3.F. Gas-Surface Energy Exchange $\quad 209$

5.3.D. Time Dependent Reactivity 211

$\begin{array}{ll}\text { 5.4. Summary } & 211\end{array}$

$\begin{array}{ll}\text { Figures and Tables } & 213\end{array}$ 


\section{References}


1.

\section{Research Motivation}

Catalysis impacts many aspects of our everyday lives. For example, catalysis in the catalytic converter of motor vehicles allows carbon monoxide, $\mathrm{NO}_{\mathrm{x}}$, and other toxic exhaust chemicals to be converted into safer and more stable gases such as carbon dioxide, oxygen, nitrogen, and water. Catalysis can aid in chemical transformations two ways, by lowering the energetic barrier to reaction and by selectively guiding the reactants to a desired product.

Arguably, the greatest invention of the $20^{\text {th }}$ century was the development of the HaberBosch process, a means of creating synthetic ammonia from hydrogen and nitrogen through the catalytic use of potassium doped iron nanoparticles. The carrying capacity of the world is only $\sim 3$ billion people if every bit of arable land is used and organic wastes are recycled, meaning the Haber process has allowed for the population to more than double past that natural limit. As the population of the world continues to expand, and demand on the world's natural resources increases, an important challenge facing mankind in the $21^{\text {st }}$ century will be to transition from an economy based on low-priced fossil fuels to one reliant on alternative and renewable sources of energy driving more energy efficient processes in ways that can curb or reduce climate changing $\mathrm{CO}_{2}$ emissions. To meet this challenge, it would be helpful to be able to scientifically design next generation catalysts and catalytic processes based on a microscopic level of understanding of reactivity at catalytic surfaces.

Steam reforming of natural gas (95\% methane plus other light alkanes) in which gas and water are combined over a metal catalyst to produce synthesis gas, a mixture of hydrogen and $\mathrm{CO}$, is a vital industrial process. Currently, the dissociation of the initial $\mathrm{C}-\mathrm{H}$ bond of methane is 
believed to be the rate-limiting step ${ }^{1-5}$ in the reforming process, one in which the $438 \mathrm{~kJ} / \mathrm{mol} \mathrm{C}-\mathrm{H}$ bond of gas-phase methane is lowered to $65 \mathrm{~kJ} / \mathrm{mol}$ during dissociative chemisorption on a nickel catalyst ${ }^{6}$. Synthesis gas can then be used to create a wide range of industrially important products through a variety of chemical reactions (e.g. Fischer Tropsch synthesis to liquid hydrocarbons fuels, Mobil 1 synthetic motor oil, etc). Additionally, steam reforming of natural gas provides the industrial supply of $\mathrm{H}_{2}$ at the lowest cost. Following steam reforming, the water gas shift can be used to produce an additional equivalent of hydrogen by combining the CO produced from steam reforming with additional water.

$$
\begin{array}{ll}
\mathrm{CH}_{4}+\mathrm{H}_{2} \mathrm{O} \stackrel{\mathrm{Ni}}{\longrightarrow} 3 \mathrm{H}_{2}+\mathrm{CO} & \left(\Delta_{r} \mathrm{H}_{298.15 \mathrm{~K}}^{o}=206 \mathrm{~kJ} / \mathrm{mol}\right) \\
\mathrm{CO}+\mathrm{H}_{2} \mathrm{O} \stackrel{\mathrm{Ni}}{\longrightarrow} \mathrm{H}_{2}+\mathrm{CO}_{2} & \left(\Delta_{r} \mathrm{H}_{298.15 \mathrm{~K}}^{o}=-41 \mathrm{~kJ} / \mathrm{mol}\right) \\
\mathrm{CH}_{4}+2 \mathrm{H}_{2} \mathrm{O} \stackrel{\mathrm{Ni}}{\longrightarrow} 4 \mathrm{H}_{2}+\mathrm{CO}_{2} & \left(\Delta_{r} \mathrm{H}_{298.15 \mathrm{~K}}^{o}=165 \mathrm{~kJ} / \mathrm{mol}\right)
\end{array}
$$

Particularly for mobile applications, there has been interest in autothermal catalytic partial oxidation of alkanes into desirable products using short contact time frit reactors typically employing a Pt or Rh catalyst,

$$
\begin{array}{ll}
\mathrm{CH}_{4}+\frac{1}{2} \mathrm{O}_{2} \longrightarrow 2 \mathrm{H}_{2}+\mathrm{CO} & \left(\Delta_{r} \mathrm{H}_{298.15 \mathrm{~K}}^{o}=-38 \mathrm{~kJ} / \mathrm{mol}\right)^{7} \\
\mathrm{C}_{2} \mathrm{H}_{6}+\mathrm{O}_{2} \longrightarrow 3 \mathrm{H}_{2}+2 \mathrm{CO} & \left(\Delta_{r} \mathrm{H}_{298.15 \mathrm{~K}}^{o}=-136 \mathrm{~kJ} / \mathrm{mol}\right) \\
\mathrm{C}_{2} \mathrm{H}_{6}+\frac{1}{2} \mathrm{O}_{2} \longrightarrow \mathrm{C}_{2} \mathrm{H}_{4}+\mathrm{H}_{2} \mathrm{O} & \left(\Delta_{r} \mathrm{H}_{298.15 \mathrm{~K}}^{o}=-105 \mathrm{~kJ} / \mathrm{mol}\right)^{8,9}
\end{array}
$$


With the current boom in natural gas availability in the United States through advances in fracking shale beds, methane and other light alkanes will be increasingly available to the chemical industry which will make optimizing their catalytic transformations economically rewarding.

The highly chaotic and multifaceted nature of industrial catalytic reactions makes it difficult to discern the properties that influence reactivity. In contrast to the hellish and difficult to probe conditions of typical industrial catalytic processes (e.g. $100 \mathrm{~atm}, 500{ }^{\circ} \mathrm{C}$ over metal nanoparticles on an oxide support), surface science studies investigate reactivity under highly characterizable, ultra-high vacuum (UHV) conditions, $\mathrm{p}<10^{-10}$ Torr, with well-defined gas fluences impinging on single crystal metal surfaces. Due to the disparities in pressure, energy, and material conditions between surface science and catalysis, difficulties arise in linking the information gained in surface science experiments to equivalent real world catalysis problems occurring at elevated temperatures and pressures. Bridging these condition gaps (e.g. the pressure gap spanning 15 orders of magnitude) has been a long standing goal, and it would be helpful to develop models that can quantitatively reproduce surface science and high pressure catalysis experiments in a unified and consistent manner.

One widespread technique used to guide catalyst discovery and optimization is electronic structure calculations, which tend to suffer either from prohibitive computation costs for convergent theories (ab initio coupled cluster theory) or from a lack of optimization for surface reactivity (GGA-DFT), such that conflicting results often arise depending on the basis set and method used. Instead, we have developed a precursor mediated microcanonical trapping (PMMT) kinetics model ${ }^{10-13}$ of gas-surface reactivity based on microcanonical transition state theory, which can be used to predict a diverse range of phenomena once several parameters have been determined based on analysis of some experimental data. It has been possible to replicate 
dissociative chemisorption results from varied surface science experiments using supersonic beams, effusive beams, laser-pumping of single eigenstates, thermal bulb, and laser induced associative desorption, while also providing insight into the relative importance of different gas and surface degrees of freedom in promoting reactivity. The PMMT is a statistical model into which dynamical biases can be built related to the efficiency by which some kinds of energy (e.g. vibrational) promote reactivity. The PMMT model is able to extract important characteristics of the transition state (e.g. reaction threshold energy) from analysis of experiments that can provide benchmarks for electronic theory calculations and can be used to make predictions about reactivity under varied other conditions. In this dissertation, the PMMT model has been applied to the dissociative chemisorption and associative desorption of $\mathrm{H}_{2}$ on $\mathrm{Cu}(111), \mathrm{CH}_{4}$ on $\mathrm{Pt}(111)^{13}$, ${ }^{14}, \mathrm{Ru}(0001), \mathrm{Ni}(111), \mathrm{Ni}(100), \operatorname{Ir}(111)$, and small alkanes $\mathrm{C}_{\mathrm{n}} \mathrm{H}_{2 \mathrm{n}+2}$ on $\operatorname{Pt}(111)^{15}, \mathrm{n}=1-3$. The introduction of dynamical biases and tunneling to the PMMT model was accomplished during this thesis work. Attention has been focused on the effect of dynamics on gas-surface reactivity and on how to relate the d-PMMT parameters to transition state characteristics calculated by electronic structure theory. At least for methane reactivity on transition metal surfaces, the Polanyi rules ${ }^{16,17}$ for dynamically shifting translational /vibrational energy reactive preferences with early/late transition state barrier position seem to apply. 


\section{References:}

1. Wei, J. M.; Iglesia, E., Mechanism and site requirements for activation and chemical conversion of methane on supported Pt clusters and turnover rate comparisons among noble metals. J. Phys. Chem. B 2004, 108, (13), 4094-4103.

2. Wei, J. M.; Iglesia, E., Reaction Pathways and Site Requirements for the Activation and Chemical Conversion of Methane on Ru-Based Catalysts. J. Phys. Chem. B 2004, 108, 72537262.

3. Wei, J. M.; Iglesia, E., Isotopic and kinetic assessment of the mechanism of reactions of $\mathrm{CH} 4$ with $\mathrm{CO} 2$ and $\mathrm{H} 2 \mathrm{O}$ to form synthesis gas and carbon on nickel catalysts. J. Catal. 2004, 224, 370-383.

4. Wei, J. M.; Iglesia, E., Structural requirements and reaction pathways in methane activation and chemical conversion catalyzed by rhodium. J. Catal. 2004, 225, 116-127.

5. Wei, J. M.; Iglesia, E., Structural and Mechanistic Requirements for Methane Activation and Chemical Conversion on Supported Iridium Clusters. Angew. Chem. Int. Ed. 2004, 43, 36853688 .

6. Nielsen, B. O.; Luntz, A. C.; Holmblad, P. M.; Chorkendorff, I., Activated Dissociative Chemisorption of Methane on $\mathrm{Ni}(100)$ - a Direct Mechanism under Thermal Conditions. Catal. Lett. 1995, 32, (1-2), 15-30.

7. Hickman, D. A.; Schmidt, L. D., Production of Syngas by Direct Catalytic Oxidation of Methane. Science 1993, 259, (5093), 343-346.

8. Henning, D. A.; Schmidt, L. D., Oxidative dehydrogenation of ethane at short contact times: species and temperature profiles within and after the catalyst. Chem. Eng. Sci. 2002, 57, (14), 2615-2625.

9. Goetsch, D. A.; Schmidt, L. D., Microsecond catalytic partial oxidation of alkanes. Science 1996, 271, (5255), 1560-1562.

10. Bukoski, A.; Abbott, H. L.; Harrison, I., Microcanonical unimolecular rate theory at surfaces. III. Thermal dissociative chemisorption of methane on $\operatorname{Pt}(111)$ and detailed balance. $J$. Chem. Phys. 2005, 123, (9), 094707.

11. Abbott, H. L.; Bukoski, A.; Harrison, I., Microcanonical unimolecular rate theory at surfaces. II. Vibrational state resolved dissociative chemisorption of methane on $\mathrm{Ni}(100) . J$. Chem. Phys. 2004, 121, (8), 3792-3810.

12. Bukoski, A.; Blumling, D.; Harrison, I., Microcanonical unimolecular rate theory at surfaces. I. Dissociative chemisorption of methane on $\operatorname{Pt}(111)$. J. Chem. Phys. 2003, 118, (2), 843-871.

13. Donald, S. B.; Harrison, I., Dynamically biased RRKM model of activated gas-surface reactivity: vibrational efficacy and rotation as a spectator in the dissociative chemisorption of $\mathrm{CH}_{4}$ on Pt(111). Phys. Chem. Chem. Phys. 2012, 14, (5), 1784-1796.

14. Navin, J. K.; Donald, S. B.; Tinney, D. G.; Cushing, G. W.; Harrison, I., Communication: Angle-resolved thermal dissociative sticking of $\mathrm{CH}_{4}$ on $\mathrm{Pt}(111)$ : Further indication that rotation is a spectator to the gas-surface reaction dynamics. J. Chem. Phys. 2012, 136, 061101.

15. Cushing, G. W.; Navin, J. K.; Donald, S. B.; Valadez, L.; Johanek, V.; Harrison, I., C-H Bond Activation of Light Alkanes on Pt(111): Dissociative Sticking Coefficients, Evans-Polanyi Relation, and Gas-Surface Energy Transfer. J. Phys. Chem. C 2010, 114, (40), 17222-17232.

16. Polanyi, J. C., Some concepts in reaction dynamics. Acc. Chem. Res. 1972, 5, (5), 161168.

17. Polanyi, J. C.; Wong, W. H., Location of Energy Barriers. I. Effect on the Dynamics of Reactions A + BC. J. Chem. Phys. 1969, 51, 1439-1450. 


\title{
RRKM Simulation of Hydrogen Dissociation on $\mathrm{Cu}(111)$ :
} Addressing Dynamical Biases, Surface Temperature, and

\section{Tunneling}

\begin{abstract}
The effects of dynamics, surface temperature, and tunneling on the dissociative chemisorption of hydrogen on $\mathrm{Cu}(111)$ are explored using a dynamically-biased precursor mediated microcanonical trapping (d-PMMT) model. Transition state vibrational frequencies were taken from recent GGA-DFT electronic structure calculations and the model's few remaining parameters were fixed by optimizing simulations to a limited number of quantum-state-resolved associative desorption experiments. The d-PMMT model reproduces a diverse variety of dissociative chemisorption and associative desorption experimental results, and, importantly, largely captures the surface temperature dependence of quantum-state-resolved dissociative sticking coefficients. Molecular translational energy parallel to the surface was treated as a spectator degree of freedom. The efficacy of molecular rotational energy to promote dissociation, relative to normal translational energy, varied monotonically from $-45 \%$ to $33 \%$ as the rotational energy increased. The molecular vibrational and surface phonon efficacies were $60 \%$. Efficacies did not vary with isotope change from $\mathrm{H}_{2}$ to $\mathrm{D}_{2}$. The thermal dissociative sticking coefficient for $\mathrm{H}_{2} / \mathrm{Cu}(111)$ is predicted to vary as $S(T)=S_{0} \exp \left(-E_{a} / R T\right)$ where $S_{0}=0.075$ and $E_{a}=49.2 \mathrm{~kJ} / \mathrm{mol}$ over the $300 \mathrm{~K} \leq T \leq 1000 \mathrm{~K}$ temperature range. Dynamical effects are significant and suppress $S(T)$ by $\sim 2$ orders of magnitude as compared to statistical expectations. For thermal dissociative chemisorption of $\mathrm{H}_{2} / \mathrm{Cu}(111)$ at $1000 \mathrm{~K}$, a temperature of catalytic interest, normal translational energy is calculated to provide $74 \%$ of the energy necessary to react, surface phonons $17 \%$, molecular rotation $5 \%$, and vibration $4 \%$. Tunneling is calculated to account for $13 \%$ of $S(T)$ at $1000 \mathrm{~K}$, but more than $50 \%$ at temperatures below $400 \mathrm{~K}$.
\end{abstract}


2.1. Introduction

The dissociative chemisorption of hydrogen on $\mathrm{Cu}(111)$ is an important model system for gas-surface reactivity. Due to its relative simplicity, $\mathrm{H}_{2}$ dissociative chemisorption and recombinative desorption on $\mathrm{Cu}(111)$ is often considered the quintessential system for both experimental and theoretical surface scientists to study. ${ }^{1-3}$ It is perhaps the easiest catalytic system to model theoretically since it involves the smallest diatomic molecule and a closed shell, first row, transition metal surface. It has also been a target system for theorists because there is a wealth of experimental data available for comparison. ${ }^{3-17}$ With the recent push toward alternative energy sources (e.g., $\mathrm{H}_{2}$ fuel cells), understanding the reactivity of the $\mathrm{H}-\mathrm{H}$ bond has become an increasingly important problem. There is still no consensus among experimentalists and theorists on the activation energy of $\mathrm{H}_{2}$ dissociation on $\mathrm{Cu}(111)$ and the role of the surface has largely been neglected even in the most recent, high level, quantum dynamical calculations. ${ }^{18-27}$

Early experiments by Rendulic and coworkers measured the dissociative sticking coefficient of hydrogen on $\mathrm{Cu}(111)$ as a function of molecular translational energy by seeding the molecular beam at fixed internal energy, i.e. at constant molecular beam nozzle temperature. . $^{28}$ Molecular beam experiments by Auerbach and coworkers have shown that both vibrational and normal translational energy promote the dissociative sticking of $\mathrm{H}_{2}$ and $\mathrm{D}_{2}$ on $\mathrm{Cu}(111){ }^{6,7,9,10}$ Interestingly, the kinetic isotope effect in these experiments is very small. Detailed balance holds at thermal equilibrium between the flux of associatively desorbing molecules and the flux of molecules that successfully dissociatively chemisorb when they impinge on the surface. Consequently, by detailed balance, the desorption product state and energy distributions must be the same as those distributions of the successfully reacting molecules that are culled from the thermal distribution of all molecules striking the surface at thermal equilibrium. Thermallydriven associative desorption (the time reversal of dissociative chemisorption) experiments, 
interpreted through the principle of detailed balance, have shown that molecular vibrational and rotational energies are not as efficacious in promoting dissociative chemisorption as molecular translational energy directed along the surface normal. ${ }^{6,7}$ Recombinative desorption experiments allowed quantum state resolved reactivity to be explored by measuring ro-vibrationally stateresolved translational energy distributions of desorbing molecules. ${ }^{6,7,11}$ Auerbach proposed that molecules approaching the surface in different ro-vibrational states sampled different distributions of dissociation barriers. ${ }^{6,7}$ More recent associative desorption experiments have shown that rotational alignment of the desorbing molecules decreases with increasing translational energy and increases with increasing rotational energy, indicative of a greater importance of dynamical steering at low energies in dissociative sticking. ${ }^{14,}{ }^{15}$ Murphy and Hodgson derived eigenstate-resolved, surface temperature dependent sticking coefficients and effective activation energies " $E_{a}\left(T_{s} ; E_{n}, v, J\right)$ " based on associative desorption experiments for $\mathrm{H}_{2}$ and $\mathrm{D}_{2}$ from $\mathrm{Cu}(111) .{ }^{16}$ These effective activation energies were derived by performing Arrhenius fits to relative sticking coefficients, $S_{r e l}\left(T_{s} ; E_{n}, v, J\right)$, versus surface temperature. Plotting $\left\langle E_{t}(\vartheta)\right\rangle$ versus $E_{n}$ revealed a slope of approximately -1 . On this basis, Hodgson concluded the efficacy of surface energy to promote reactivity was very similar to that of normal translational energy.

Theoretical investigations of $\mathrm{H}_{2}$ dissociative chemisorption on $\mathrm{Cu}(111)$ are plentiful, but rarely have direct quantitative comparisons been made to experimental results. Gro $\beta$ et al. calculated dissociative sticking coefficients based on 2D and 5D generalized gradient approximation (GGA) - density function theory (DFT) calculations and compared them to relative sticking coefficients measured by associative desorption of $\mathrm{H}_{2} \cdot{ }^{18}$ These calculated sticking coefficients underestimated the experimental sticking by a $\sim 19 \mathrm{~kJ} / \mathrm{mol}$ shift in the translational energy axis which led authors to suggest a barrier height of $E_{0} \approx 48 \mathrm{~kJ} / \mathrm{mol}$ instead 
of the DFT value of $E_{0}=70 \mathrm{~kJ} / \mathrm{mol}$ on which the dynamical results had been calculated. Direct comparison was also made to the mean translational energy, $\left\langle E_{t}(\vartheta)\right\rangle$, and angular yield distributions measured by Comsa and David using a quadrupole mass spectrometer and a pseudorandom chopper to obtain time-of-flight spectra. ${ }^{17}$ Reasonable agreement was obtained for the mean translational energies of the desorbing molecules, but the theoretical $\left\langle E_{t}(\vartheta)\right\rangle$ increased rather than decreased with $\vartheta$. The calculated angular desorption yield distribution was considerably sharper than the experimental $\cos ^{\mathrm{n}} \theta$ fit [i.e., $\mathrm{n}_{\text {theory }}=25$ versus $\mathrm{n}_{\text {expt }}=8$ at $T_{s}=1000$ $\mathrm{K}$ for $\left.\mathrm{D}_{2} / \mathrm{Cu}(100)\right]$.

DFT calculations yielding a 6-D potential energy surface performed by Norskøv and coworkers found a classical barrier height of $E_{c l} \approx 48 \mathrm{~kJ} / \mathrm{mol} .{ }^{20}$ Later calculations by Sakong and Gro $\beta$, found a similar classical barrier height for dissociative adsorption of $E_{c l}=48.5 \mathrm{~kJ} / \mathrm{mol} .{ }^{19}$ They found the transition state occurred when $\mathrm{H}_{2}$ is parallel to the surface and centered over a bridge site. More recent $6 \mathrm{D}$ wave packet dynamical calculations, which included all the molecular degrees of freedom, examined the behavior of eigenstate-resolved $\mathrm{H}_{2}$ and $\mathrm{D}_{2}$ scattering from $\mathrm{Cu}(111)$, using a potential with $E_{0}$ varying from 52 to $69 \mathrm{~kJ} / \mathrm{mol} .{ }^{22,23}$ Dai and Light explored the role of steric effects on the dissociation of hydrogen and determined that "helicopter" rotational motion (i.e., angular momentum vector $\vec{J}$ along the surface normal) promotes reaction more than "cartwheel" rotational motion (i.e., $\vec{J}$ in the surface plane). ${ }^{26,27}$

Although most theoreticians have neglected the role of the surface degrees of freedom in the reactivity of hydrogen on $\mathrm{Cu}$, Wang et al. have studied the effects of surface temperature. ${ }^{24,25}$ They attempted to replicate the recombinative desorption experimental data reported by Murphy and Hodgson ${ }^{16}$ using 4-D wave packet calculations and a single Einstein surface oscillator to treat the surface. ${ }^{24}$ The 4 -D potential energy surface (PES) employed by this method had a reaction 
threshold energy of $E_{c l}=67.5 \mathrm{~kJ} / \mathrm{mol}$ for broadside approach. Holloway's analysis gave a slope $\partial " E_{a}\left(T_{s}\right) " / \partial E_{t}=-0.86$ for $\mathrm{D}_{2}(v=1, J=0)$ for translational energies between 30.1 to 35.2 $\mathrm{kJ} / \mathrm{mol}$ changing to a slope of $\partial^{\prime \prime} E_{a}\left(T_{s}\right) " / \partial E_{t}=-0.218$ from 35.2 to $43.4 \mathrm{~kJ} / \mathrm{mol}$ (c.f. experimental slopes of -0.92 and -0.97 for $\mathrm{H}_{2}$ and $\mathrm{D}_{2}$ in $v=0$ for normal translational energies from 5 to $60 \mathrm{~kJ} / \mathrm{mol}$. Holloway and coworkers ultimately qualitatively argued that any system with large coupling between the surface and the adsorbate should obey Arrhenius behavior with a slope " $E_{a}\left(T_{s}\right)$ " versus $E_{\mathrm{n}}$ of approximately $-1 .^{24}$

The effect of dynamical steering or orientational hindering has been explored for $\mathrm{H}_{2}$ on $\mathrm{Cu}(111)$ using a variety of quantum and classical dynamics techniques. ${ }^{29}, 30$ This mechanism proposes that molecules rotating quickly (i.e., in high $J$ states) will not remain in the preferred broadside geometry as they approach the surface and, therefore, will have lower sticking coefficients. Calculations including only the cartwheel rotational motion were said to give qualitative agreement with the behavior of the experimental $\left\langle E_{t}(J)\right\rangle$ of desorbing molecules (discussed above). ${ }^{30}$ Since quantum and classical dynamics simulations gave similar results for the dissociation as a function of energy, quantum effects, such as tunneling, do not seem to play a large role in the reactivity of hydrogen on copper. ${ }^{29}$

In a recent theoretical advance, a specific reaction parameter, density functional theory, potential energy surface (SRP-DFT PES) for $\mathrm{H}_{2} / \mathrm{Cu}(111)$ was optimized to give chemically accurate agreement with some experimental data based on six-dimensional (6D) dynamical calculations that addressed all molecular degrees of freedom but assumed a static $\mathrm{Cu}$ surface. ${ }^{31-33}$ Although several dynamical approaches towards treating the effects of surface thermal energy on reactivity are available, ${ }^{34-36}$ none $^{37}$ have quantitatively accounted for Hodgson's surface 
temperature dependent quantum-state-resolved $\mathrm{H}_{2} \quad \& \quad \mathrm{D}_{2} / \mathrm{Cu}(111)$ associative desorption observations ${ }^{16}$ that have been used to argue that surface thermal energy and normal translational energy have similar efficacies in promoting dissociative chemisorption. Here, we explore an alternative, high dimensional approach for analyzing and predicting experimental data based on a dynamically-biased precursor mediated microcanonical trapping ${ }^{38-40}$ (d-PMMT) model. This model employs transition state properties derived from the SRP-DFT PES and is largely consistent with Hodgson's observations. Its few parameters, optimized to a limited set of experiments, predominantly describe dynamical deviations from statistical reactive behavior. The importance of dynamics to the gas-surface reactivity could be readily assessed by setting the dPMMT model's dynamical parameters to limiting statistical values that recover a statistical (s-) PMMT model of the reactivity. In this paper, the d-PMMT model is used to estimate the impact of dynamics, surface phonons, and tunneling on hydrogen/ $\mathrm{Cu}(111)$ dissociative chemisorption, in advance of more sophisticated, higher dimensional, dynamical calculations in at least 7 dimensions.

\section{2. d-PMMT Model}

The d-PMMT model has been described elsewhere in the context of the activated dissociative chemisorption of $\mathrm{CH}_{4}$ on $\mathrm{Pt}(111) \cdot{ }^{38-40}$ Figure 1 provides a schematic depiction of the PMMT model for $\mathrm{H}_{2} / \mathrm{Cu}(111)$. Briefly, gas-surface collision complexes comprised of an incident molecule and a few local surface oscillators are assumed to have their exchangeable energy microcanonically randomized if their pooled energy is sufficient to react such that access to the strongly state-mixing regions of the reactive PES near the transition state is guaranteed. These precursor complexes (PCs) are effectively trapped in the vicinity of the physisorption well located between the transition states for desorption and dissociative chemisorption (reaction). The PCs 
formed are assumed to go on to desorb or react with Rice-Ramsperger-Kassel-Marcus (RRKM) ${ }^{41}$ rate constants,

$$
k_{i}\left(E^{*}\right)=\frac{W_{i}\left(E^{*}\right)}{h \rho\left(E^{*}\right)}
$$

where $E^{*}$ is the active exchangeable energy whose zero occurs for the reactants at rest at infinite separation, $W_{i}\left(E^{*}\right)$ is the sum of states for transition state $i=D, R$ with threshold energies $E_{D}^{*}=0$ and $E_{R}^{*}=E_{0}$ (see Fig. 1 ), $\rho\left(E^{*}\right)$ is the PC density of states, and $h$ is Planck's constant.

Applying the steady state approximation to the $\mathrm{H}_{2(\mathrm{p})}$ coverage of the Fig. 1 kinetics scheme,

$$
H_{2(g)} \underset{k_{D}\left(E^{*}\right)}{\stackrel{F_{0} f\left(E^{*}\right)}{\rightleftarrows}} H_{2(p)} \stackrel{k_{R}\left(E^{*}\right)}{\longrightarrow} 2 H_{(c)}
$$

yields an expression for the experimental dissociative sticking coefficient,

$$
S=\int_{0}^{\infty} S\left(E^{*}\right) f\left(E^{*}\right) d E^{*}
$$

where $S\left(E^{*}\right)$ is the microcanonical sticking coefficient,

$$
S\left(E^{*}\right)=\frac{k_{R}\left(E^{*}\right)}{k_{R}\left(E^{*}\right)+k_{D}\left(E^{*}\right)}=\frac{W_{R}\left(E^{*}\right)}{W_{R}\left(E^{*}\right)+W_{D}\left(E^{*}\right)}
$$

and $f\left(E^{*}\right)$ is the probability distribution for forming a PC with exchangeable energy $E^{*}$, which is calculated by convolution over the molecular and surface energy distributions describing the particular experimental conditions of interest. The experimental sticking coefficient is the average of the microcanonical sticking coefficient over the experimental probability of forming a PC with 
energy $E^{*}$. The microcanonical sticking coefficient is the ratio of the number of open channels to react to the total number of open channels to either react or desorb. $S\left(E^{*}\right)$ is a statistical quantity derived solely from the quantum structure of the transition states.

Experimental dissociative sticking coefficients for hydrogen on $\mathrm{Cu}(111)$ are found to scale with the translational energy directed along the surface normal, ${ }^{10} E_{n}=E_{t} \cos ^{2} \vartheta$, and are independent of the translational energy directed parallel to the surface, $E_{t} \sin ^{2} \vartheta$. Consequently, translational motion parallel to the surface is taken to be a spectator degree of freedom.

Dynamical biases are introduced ${ }^{38}$ only during formulation of the PC exchangeable energy available to surmount the reaction barrier,

$$
E^{*}=E_{n}+\eta_{v} E_{v}+\eta_{r} E_{r}+\eta_{s} E_{s}
$$

where $E_{n}, E_{v}, E_{r}, E_{s}$ are the normal translational energy, vibrational energy, rotational energy, and surface energy, respectively, and the $\eta_{i}$ are efficacies for different kinds of energy to promote reaction relative to $E_{n}$. For example, the vibrational efficacy is,

$$
\left.\left.\eta_{v} \equiv \frac{\partial S}{\partial E_{v}}\right|_{E_{j \neq v}} \frac{\partial S}{\partial E_{n}}\right|_{E_{j \neq n}} ^{-1}
$$

which may be measured experimentally in quantum-state-resolved dissociative sticking coefficient experiments as, ${ }^{42}$

$$
\eta_{v}=\left.\frac{\Delta E_{n}}{\Delta E_{v}}\right|_{\Delta S_{v}}
$$


where $\Delta S_{v}$ is the change in $S$ accompanying a $\Delta E_{v}$ increase in vibrational energy at some particular $E_{n}$, and $\Delta E_{n}$ is the change in $E_{n}$ required to gain the same $\Delta S_{v}$ change in $S$ for molecules maintained in the lower energy vibrational state. The rotational efficacy may be measured similarly, ${ }^{43}$ but the surface efficacy cannot. For hydrogen dissociative chemisorption on $\mathrm{Cu}(111)$, efficacy values have been determined from thermal associative desorption experiments interpreted on the basis of detailed balance arguments. ${ }^{6,7}$

The flux distribution for creation of PCs with exchangeable energy $E^{*}$ is calculated by convolution over the active energy distributions of the incident molecules and the surface energy distribution of the local surface oscillators describing the particular experiment,

$$
\begin{aligned}
f\left(E^{*}\right)=\int_{0}^{E^{*}} f_{n}\left(E_{n}\right) \int_{0}^{\left(E^{*}-E_{n}\right) / \eta_{v}} f_{v}\left(E_{v}\right) \int_{0}^{\left(E^{*}-E_{n}-\eta_{v} E_{v}\right) / \eta_{r}} f_{r}\left(E_{r}\right) \\
\quad \times f_{s}\left(\eta_{s}^{-1}\left(E^{*}-E_{n}-\eta_{v} E_{v}-\eta_{r} E_{r}\right)\right) d E_{r} d E_{v} d E_{n}
\end{aligned}
$$

The surface energy distribution, $f_{s}\left(E_{s}\right)$, is calculated for the $s$ surface oscillators making up each PC under the assumption the oscillators all vibrate at the mean phonon frequency of $\mathrm{Cu}$, $v_{s}=\frac{3}{4} k_{B} \theta_{\text {Debye }} / h c=175 \mathrm{~cm}^{-1}$. Hydrogen vibrational and rotational energy levels are calculated as $\quad E_{v}(\mathrm{v})=h c\left[\mathrm{v} \omega_{e}-\mathrm{v}(\mathrm{v}+1) \omega_{e} \chi_{e}\right] \quad$ and $\quad E_{r}(J)=h c\left[B_{e} J(J+1)-D_{e} J^{2}(J+1)^{2}\right] \mathrm{using}$ Herzberg's spectroscopic constants including anharmonic and centrifugal corrections. ${ }^{44}$ The Beyer-Swinehart-Stern-Rabinovitch ${ }^{45}$ algorithm is used to calculate sums and densities of states as necessary for Eqs. (2.4) \& (2.8).

Over the ultrafast desorption lifetimes of PCs formed at reactive energies, nuclear spin flips are assumed not to occur and so the manifold of rotational states available for desorption depends on the ortho or para spin statistics of the incident molecules. For experiments involving 
molecules with thermally populated rotational states, dissociative sticking coefficients for the ortho and para components ${ }^{44}$ of the incident gas flux are calculated separately (e.g, with paraspecific $f_{p}\left(E^{*}\right)$ and $S_{p}\left(E^{*}\right)$ in Eq.(3)) and then averaged using the "high temperature" statistical ratios, appropriate to room temperature cylinders of gas, of 3:1 for $\mathrm{H}_{2}$ and 2:1 for $\mathrm{D}_{2}$ to calculate the experimental $S$. Internal state distribution for hydrogen incident from supersonic molecular beams are modeled assuming the beam nozzle temperature, $T_{N}$, sets the molecular vibrational and rotational temperatures as $T_{v}=T_{N}$ and $T_{r}=0.9 T_{N}$, respectively. ${ }^{6}$

The microcanonical sticking coefficient, $S\left(E^{*}\right)$ of Eq.(2.4), depends on the properties of the transition states for desorption and reaction and is formulated statistically with no dynamical biasing (i.e., as appropriate for a s-PMMT model with $\left\{\eta_{i}\right\}=1$ ). The transition state for PC desorption is taken to occur with the hydrogen molecule freely rotating and vibrating in the gasphase far from the $s$ surface oscillators left behind on the surface. The reaction co-ordinate for desorption is translational motion along the surface normal and so this degree of freedom, along with the two spectator translations parallel to the surface, are missing from $W_{D}^{\dagger}\left(E^{*}\right)$. Transition state properties for hydrogen dissociative chemisorption on $\mathrm{Cu}(111)$ were generously derived from the recent SRP-DFT-PES ${ }^{31}$ by Krishnamohan, Díaz, Olsen, and Kroes and these properties appear in tabled form for reaction with $\mathrm{H}_{2}, \mathrm{D}_{2}$, and $\mathrm{HD}$ within the electronic supplementary information (ESI). The reaction co-ordinate for hydrogen dissociative chemisorption is a mixture of molecular bond extension and motion towards the surface ${ }^{31}$ and its vibrational mode frequency is imaginary. This reaction co-ordinate mode, along with the two spectator translational modes in the surface plane, are missing from $W_{R}\left(E^{*}\right)$. Calculation of $S\left(E^{*}\right)$ requires specification of $\left\{E_{0}, s\right\}$. Typically, $\left\{E_{0}, s\right\}$ are free variables of a PMMT model and chosen by optimization of PMMT simulations to experiments. The apparent threshold energy for reaction, 
$E_{0}=E_{c}+\left(Z_{T S}-Z_{R e}\right)$, is the classical barrier, $E_{c}$, plus the difference in vibrational zero-point energies between the transition state and reactants. For the SRP-DFT-PES, $E_{c}=60.6 \mathrm{~kJ} / \mathrm{mol}$ which yields $E_{0}=56.5 \mathrm{~kJ} / \mathrm{mol}, 56.8 \mathrm{~kJ} / \mathrm{mol}$, and $57.7 \mathrm{~kJ} / \mathrm{mol}$ for $\mathrm{H}_{2}, \mathrm{HD}$, and $\mathrm{D}_{2}$ on $\mathrm{Cu}(111)$, respectively, based on the vibrational frequencies listed in the ESI. Here, we treat $E_{0}$ for $\mathrm{H}_{2} / \mathrm{Cu}(111)$ as a parameter and consistently list only its value below even when discussing results for other isotopes. Threshold energies for other isotopes were calculated from the $\mathrm{H}_{2} / \mathrm{Cu}(111)$ value using the SRP-DFT-PES transition state vibrational frequencies and Herzberg's expressions $^{44}$ for the experimentally observed vibrational frequencies of gas-phase hydrogen. The $\mathrm{H}_{2} / \mathrm{Cu}(111)$ dissociative chemisorption SRP-DFT-PES ${ }^{31,} 32$ is classically exothermic by 31.4 $\mathrm{kJ} / \mathrm{mol}$ (i.e., with no zero-point corrections).

To account for tunneling through the barrier to dissociative chemisorption, the RRKM rate constant for dissociative chemisorption was written in its generalized form as, ${ }^{46}$

$$
\begin{aligned}
k_{R}(E) & =\frac{W_{R}(E)}{h \rho(E)}=\int_{0}^{E} p\left(\varepsilon_{t}\right) k_{R}\left(E, \varepsilon_{t}\right) d \varepsilon_{t} \\
& =\frac{1}{h \rho(E)} \int_{0}^{E} p\left(\varepsilon_{t}\right) \rho_{R}\left(E-\varepsilon_{t}\right) d \varepsilon_{t}
\end{aligned}
$$

where $E=E^{*}+Z_{R e}$ is the classical energy above the electronic potential energy surface whose zero is set by the well-separated reactants at $\mathrm{T}=0 \mathrm{~K}, \varepsilon_{t}$ is the translational energy along the reaction coordinate leading to separated products, $p\left(\varepsilon_{t}\right)$ is the tunneling probability, and $\rho_{R}\left(E-\varepsilon_{t}\right)$ is the density of states, excluding the reaction coordinate mode, of the reactive transition state evaluated at the energy available to populate vibrational states of the transition state complex when tunneling occurs at $\varepsilon_{t} \cdot{ }^{41,47}$ The barrier to chemisorption was approximated 
by a $1 \mathrm{D}$ Eckart potential ${ }^{48,49}$ whose height was $E_{c}=E_{0}-\left(Z_{T S}-Z_{R e}\right)$ while the curvature and exothermicity were fixed by the SRP-DFT-PES (see Fig. 2.2(a)). The analytic expression ${ }^{48}$ for the tunneling probability through a $1 \mathrm{D}$ Eckart barrier relates to $\varepsilon_{t}$ above the classical electronic potential evaluated for the well-separated reactants, without regard to zero-point energies, and so the sum of states for the reactive transition state referenced to the $E^{*}$ energy scale can be written as,

$$
W_{R}\left(E^{*}\right)=\int_{0}^{E^{*}+Z_{R e}} p\left(\varepsilon_{t}\right) \rho_{R}\left(E^{*}+Z_{R e}-\varepsilon_{t}\right) d \varepsilon_{t}
$$

where the argument of $\rho_{R}$ is the energy available to populate vibrational states of the transition state complex, taking in to account their vibrational zero-point energy. In the absence of tunneling,

$$
p\left(\varepsilon_{t}\right) \begin{cases}0, & E<E_{c l}+Z_{T S} \\ 1, & E \geq E_{c l}+Z_{T S}\end{cases}
$$

and given $\varepsilon_{t}=\varepsilon_{t}^{*}+Z_{R e}$, Eq.(2.10) reduces to the conventional non-tunneling expression,

$$
\begin{aligned}
W_{R}\left(E^{*}\right) & =\int_{E_{c l}+Z_{T S}}^{E^{*}+Z_{R e}} \rho_{R}\left(E^{*}+Z_{R e}-\varepsilon_{t}\right) d \varepsilon_{t} \\
& =\int_{E_{0}}^{E^{*}} \rho_{R}\left(E^{*}-\varepsilon_{t}^{*}\right) d \varepsilon_{t}^{*}=\int_{0}^{E^{*}-E_{0}} \rho_{R}\left(E^{*}-E_{0}-\varepsilon_{t}^{*}\right) d \varepsilon_{t}^{*} \\
& =W_{R}^{\dagger}\left(E^{*}-E_{0}\right)=\int_{0}^{E^{\dagger}} \rho_{R}^{\dagger}\left(E^{\dagger}\right) d E^{\dagger}
\end{aligned}
$$

where the arguments of the daggered quantities are referenced to the energy of the transition state above its zero-point energy, e.g., $E^{\dagger}=E^{*}-E_{0}=E-E_{c}-Z_{T S}$. To account for tunneling, it was operationally convenient to calculate the convolution of Eq. (2.10) as, 


$$
W_{R}\left(E^{*}\right)=\int_{0}^{E^{*}+Z_{R e}} p\left(\varepsilon_{t}\right) \rho_{R}^{\dagger}\left(E^{*}+Z_{R e}-Z_{T S}-\varepsilon_{t}\right) d \varepsilon_{t}
$$

using the Beyer-Swinehart algorithm for $\rho_{R}^{\dagger}\left(E^{\dagger}\right)$. Tunneling was not relevant to desorption because the desorption barrier is infinitely thick and approached asymptotically, so that $W_{D}\left(E^{*}\right)=W_{D}^{\dagger}\left(E^{*}\right)$. With sums of states calculated in these ways, $S\left(E^{*}\right)$ was evaluated. In particular simulations of experimental dissociative sticking coefficients where it was interesting to exclude tunneling pathways (i.e., by implementing the Eq.(11) Heaviside conditions on $\left.p\left(\varepsilon_{t}\right)\right), S\left(E^{*}\right)$ was reduced to $W_{R}^{\dagger}\left(E^{*}-E_{0}\right) /\left[W_{R}^{\dagger}\left(E^{*}-E_{0}\right)+W_{D}^{\dagger}\left(E^{*}\right)\right]$. Fig. 2.2(b) compares $S\left(E^{*}\right)$ evaluated with and without tunneling for $\mathrm{H}_{2} / \mathrm{Cu}(111)$.

An average relative discrepancy, ARD, between theoretical simulations of quantities and their experimental values for a particular set of experiments was defined, for example with regards to dissociative sticking coefficients, as,

$$
A R D=\left\langle\frac{\left|S_{\text {theory }}-S_{\text {expt }}\right|}{\min \left(S_{\text {theory }}, S_{\text {expt }}\right)}\right\rangle
$$

ARDs were a useful measure for evaluating how well simulations reproduced experiments.

A statistical microcanonical transition state model is recovered in the limit that $\left\{\eta_{i}\right\} \rightarrow 1$. Earlier s-PMMT models for $\mathrm{H}_{2} / \mathrm{Cu}(111)$ have been qualitatively successful in reproducing the surface temperature dependence of the reactivity, ${ }^{50,51}$ and a 3-parameter model treating rotation as a spectator ${ }^{51}$ gave fairly close quantitative agreement with other aspects of the reactivity (e.g., associative desorption angular distributions, product state distributions, isotope effects, etc.).

\subsection{Results and Discussion}


The d-PMMT model parameters $\left\{E_{0}, s, \eta_{v}, \eta_{s}, \eta_{r}\right\}$ were fixed by minimizing the ARD between simulations and experiments for the $925 \mathrm{~K}$ hydrogen/ $\mathrm{Cu}(111)$ associative desorption data of Figs $2.3 \& 2.4$, that comprise a limited subset of the available experimental data. The simulations assumed the efficacies are independent of isotope, and threshold energies for dissociative chemisorption vary only with changes in zero-point energies, $E_{0}=E_{c}+\left(Z_{T S}-Z_{R e}\right)$. The $\mathrm{H}_{2} / \mathrm{Cu}(111)$ parameters are $\left\{E_{0}=43.4 \mathrm{~kJ} / \mathrm{mol}, s=1, \eta_{v}=0.60\right.$, $\left.\eta_{s}=0.60, \quad \eta_{r}=-0.45+0.78 \operatorname{Erf}\left[0.026 E_{r}\right]\right\}$ where the rotational efficacy $\eta_{r}\left(E_{r}\right)$ is a function of the rotational energy expressed in $\mathrm{kJ} / \mathrm{mol}$ and $\mathrm{Erf}$ is the error function. The $\mathrm{H}_{2}$ threshold energy for dissociative chemisorption fixes both $E_{c}=47.5 \mathrm{~kJ} / \mathrm{mol}$ and $E_{0}\left(D_{2}\right)=44.6$ $\mathrm{kJ} / \mathrm{mol}$. All the d-PMMT calculations in the paper employ this single set of parameters and we generally list only the $\mathrm{H}_{2} / \mathrm{Cu}(111)$ values even when discussing experiments involving other isotopes.

\subsection{A. Associative Desorption at $925 \mathrm{~K}$}

\subsection{A.I. Rovibrationally-Resolved Translational Energy Distributions}

The thermally driven associative desorption of chemisorbed hydrogen atoms to form desorbing hydrogen molecules was studied experimentally at IBM's Almaden laboratories ${ }^{6,7}$ by diffusing hydrogen gas through a heated $\mathrm{Cu}(111)$ single crystal that formed the end of a pipe such that a continuous flow of reaction products could be maintained under ultrahigh vacuum conditions. For hydrogen associatively desorbing from the $\mathrm{Cu}(111)$ surface at $925 \mathrm{~K}$, Figure 2.3 provides some examples of state-resolved translational energy distributions derived from time-offlight (TOF) spectra following state-selective resonance enhanced multi-photon ionization. Figure 2.4 summarizes how the mean translational energy, $\left\langle E_{t}\right\rangle$, of the desorbing molecules varied with 
rovibrational state for the measured TOF spectra. The principle of detailed balance applied to hydrogen gas and a $\mathrm{Cu}(111)$ surface at thermal equilibrium with one another requires that the associatively desorbing flux should exactly balance the dissociatively sticking flux, even when the fluxes are specified at quantum state resolved levels of detail. ${ }^{6,52}$ With this principle, and assuming the absence of an ambient thermal gas over the experimental $\mathrm{Cu}(111)$ surface did not alter the thermal associative desorption dynamics, the d-PMMT model of dissociative chemisorption was used to calculate the associative desorption flux distributions. The model's parameters were fixed by minimizing the ARD of simulations to the experimental data of Figs. $2.3 \&$ 2.4. The state-resolved $\left\langle E_{t}\right\rangle$ data of Fig. 2.4 are reproduced by the d-PMMT model with an ARD of $2.4 \%$ and the full TOF spectra of Fig. 2.3 are also simulated quite well. Figure 2.4 provides evidence for translational activation of the dissociative chemisorption because the stateresolved $\left\langle E_{t}\right\rangle$ are considerably greater than $2 k_{B} T_{s}$. Under thermal equilibrium conditions, if the dissociative sticking coefficient was independent of $E_{t}$ then the all molecules striking the surface, including the successfully chemisorbing molecules, would have a flux-weighted Maxwell-Boltzmann distribution with $\left\langle E_{t}\right\rangle=2 k_{B} T_{s}$ and by detailed balance so too must the associatively desorbing molecules. Indeed, by detailed balance, the Fig. 4 data provide the mean translational energies required to successfully react for molecules impinging on to the surface with a Maxwell-Boltzmann distribution at temperature $T_{s}$. The reduction in the $\left\langle E_{t}\right\rangle$ required to react as the vibrational energy increases indicates the dissociative chemisorption is vibrationally activated. However, vibrational energy is not as efficacious as normal translational energy in promoting dissociative chemisorption ${ }^{6,7}$ and the d-PMMT modeling indicates a vibrational efficacy of $\eta_{v}=0.60$ 
Fig 2.4 shows that increasing the rotational energy of thermal molecules incident on the surface initially increases and then decreases the $\left\langle E_{t}\right\rangle$ required to overcome the activation barrier to dissociatively chemisorb. Consequently, the rotational efficacy is a function of the rotational energy $^{6,7}$ and the d-PMMT model finds $\eta_{r}\left(E_{r}\right)$ varies from -0.45 to 0.33 with increasing rotational energy as depicted in Fig. 2.5. The effect of $\eta_{r}\left(E_{r}\right)$ on d-PMMT simulations of translational energy distributions for associatively desorbing (or dissociatively sticking) $\mathrm{H}_{2}(\mathrm{v}=0$ ) over an extended range of rotational quantum number $J$ is shown in Fig. 2.6.

The efficacy of surface vibrational (phonon) energy to promote reactivity influences the leading and trailing edges of the state-resolved translational energy distributions (c..f., Fig. 3 with Fig. 5 of Ref. $51^{51}$ ) and the d-PMMT finds $\eta_{s}=0.60$. Interestingly, although the molecular and surface vibrational quanta differ substantially in energy (i.e., $4159 \mathrm{~cm}^{-1}\left(\mathrm{H}_{2}\right), 2992 \mathrm{~cm}^{-1}\left(\mathrm{D}_{2}\right)$ vs. $175 \mathrm{~cm}^{-1}$ (phonon)), the molecular and surface vibrational efficacies share the same value of 0.60 .

\subsection{A.II. Rovibrational Product State Distributions}

Vibrationally-resolved rotational Boltzmann plots for hydrogen associatively desorbing from $\mathrm{Cu}(111)$ at a surface temperature of $925 \mathrm{~K}$ are shown in Figure 2.7. The experimental rovibrational product state probabilities, $P_{v, J}$, derive from analysis of the REMPI TOF spectra and are normalized to 1 when summed over the range of measured $v, J$ states. ${ }^{6,7}$ Theoretical simulations of $P_{v, J}$ were made over a wider range of vibrational $(v=0-5)$ and rotational ( $J=0-26$ ) states and were normalized accordingly. Agreement between theory and experiment is quite good $(\mathrm{ARD}=47.3 \%)$, especially when considering that no product population information is embedded within the Figs 2.3-2.4 experimental data from which the d-PMMT parameters were 
fixed. An effective rotational temperature, $T_{r, \text { eff }}$, for the associatively desorbing molecules defined as,

$$
T_{r, e f f}=-k_{B}\left(\frac{\partial \ln \left[P_{v, J} / g_{n}(2 J+1)\right]}{\partial E_{r}}\right)^{-1}
$$

where $g_{n}$ is the nuclear spin degeneracy, depends on the slope of the Boltzmann plots. Fig. 2.7 shows that the effective rotational temperature varies with rotational energy and vibrational state. For vibrational ground state molecules, the calculated $T_{r, \text { eff }}$ initially increases with $E_{r}$, beginning at $650 \mathrm{~K}$ and rising to a peak of $1750 \mathrm{~K}$ near $E_{r}=40 \mathrm{~kJ} / \mathrm{mol}$, whereupon $T_{r, \text { eff }}$ monotonically diminishes with $E_{r}$ towards a limiting value of $T_{r, \text { eff }}=T_{s}=925 \mathrm{~K}$. The modulation of $T_{r, e f f}\left(E_{r}\right)$ diminishes for molecules with increasing vibrational excitation.

Vibrational product state distributions for associative desorption are provided in Table 2.I. The experimental geometry led to a polar angle of acceptance range for state-resolved molecular detection of $|\vartheta| \leq 20^{\circ}$ measured away from the direction of the surface normal. Given the desorption angular yield distribution $\propto \cos ^{9} \vartheta$ at $T_{s}=925 \mathrm{~K},{ }^{8} 46 \%$ of the desorbing molecules would have passed through the $|\vartheta| \leq 20^{\circ}$ range for state-resolved detection. Agreement between theory and experiment ${ }^{6,7}$ for the angle-constrained vibrational product state distribution is excellent save for the populations in the lowest two vibrational states of $\mathrm{D}_{2}$. The calculated product state distribution integrated over all desorption exit angles has slightly higher levels of vibrational excitation, as might be expected by detailed balance with a dissociative sticking coefficient that scales with only the normal component of the molecular translational energy. 


\subsection{B. Associative Desorption: Angular Distributions}

Angular yield distributions for thermally desorbing hydrogen and deuterium are presented in Figure 2.8. The d-PMMT simulations reproduce the experimental distributions ${ }^{8}$ fairly well with an overall ARD of $32.7 \%$. The simulations are narrower than the experimental distributions, particularly at low surface temperatures. At $925 \mathrm{~K}$, the $\mathrm{H}_{2} / \mathrm{Cu}(111)$ associative desorption angular yield distribution varies experimentally as $\cos ^{9} \vartheta$ which compares to predictions of $\cos ^{10} \vartheta$ by d-PMMT calculation and $\cos ^{25} \vartheta$ by 5-D quantum scattering calculations. ${ }^{53}$

For associatively desorbing $\mathrm{D}_{2}$ at $T_{s}=1000 \mathrm{~K}$, Fig. 2.9 compares experimental mean translational energies ${ }^{54}$ as a function of desorption angle, to detailed balance simulations of the mean energies of the successfully reacting precursor complexes (e.g., $\left\langle E_{j}(\vartheta)\right\rangle_{R}$ for degree of freedom j) formed in thermal dissociative chemisorption at this temperature. The d-PMMT $\left\langle E_{t}(\vartheta)\right\rangle_{R}$ underpredicts the experimental values across nearly all angles, yielding an ARD = $5.8 \%$. Some underprediction is expected because the experimental $\left\langle E_{t}(\vartheta)\right\rangle_{R}$ were calculated from only the fast component of the desorbing $\mathrm{D}_{2}$ time-of-flight spectra. Nevertheless, the dPMMT prediction that $\left\langle E_{t}(\vartheta)\right\rangle_{R}$ should increase slowly with $\vartheta$ to reach a broad maximum near $30^{\circ}$ before decreasing sharply at higher angles towards an asymptotic limit of $\left\langle E_{t}\left(\vartheta \rightarrow 90^{\circ}\right)\right\rangle_{R} \rightarrow 2 k_{B} T_{s}$, seems in good qualitative agreement with the experiments. Other theoretical models, such as the $1 \mathrm{D}$ Van Willigen model ${ }^{55,56}$ or $5 \mathrm{D}$ quantum scattering model ${ }^{53}$ predict that $\left\langle E_{t}(\vartheta)\right\rangle_{R}$ should monotonically increase with $\vartheta$. For a dissociative chemisorption system obeying normal energy scaling, the $\left\langle E_{t}\left(\vartheta \rightarrow 90^{\circ}\right)\right\rangle_{R} \rightarrow 2 k_{B} T_{s}$ limit should rigorously 
apply because at $\vartheta=90^{\circ}$ there is no influence of $E_{t}$ on the dissociative sticking coefficient and so the successfully reacting molecules at that angle must have the same translational energy distribution and mean as the incident molecules. The d-PMMT calculations of Fig. 2.9 indicate the surface and molecular internal energies become increasingly important to the gas-surface reactivity as the molecular angle of incidence increases away from the surface normal.

\subsection{Associative Desorption: Surface Temperature Dependence of $\boldsymbol{P}_{\mathrm{v}, \mathrm{J}}\left(E_{t} ; \vartheta=0^{\circ}\right)$}

Rovibrational eigenstate-resolved, translational energy distributions, $P\left(E_{t}, \vartheta=0^{\circ} ; \nu, J, T_{s}\right)$, for hydrogen associative desorption from $\mathrm{Cu}(111)$ were measured by Murphy and Hodgson ${ }^{16}$ at several surface temperatures and are shown in Figure 2.10 along with d-PMMT simulations. Hodgson used detailed balance to calculate temperature-dependent, relative dissociative sticking coefficients according to,

$$
S\left(E_{t}, \vartheta=0^{\circ} ; v, J, T_{s}\right) \propto \frac{P\left(E_{t}, \vartheta=0^{\circ} ; v, J, T_{s}\right)}{f_{M B}\left(E_{t}, T_{s}\right)}
$$

where $f_{M B}\left(E_{t}, T_{s}\right) \propto E_{t} \exp \left(-E_{t} / k_{b} T_{s}\right)$ is the flux weighted Maxwell-Boltzmann distribution for molecular translational energy at temperature $T_{s}$. These relative dissociative sticking coefficients were normalized to a common absolute scale by assuming the high translational energy limit reached was always 1 (c.f., 0.25 by Rettner and co-workers ${ }^{6}$ ). Experimentally derived, state-resolved dissociative sticking coefficients calculated in this manner ${ }^{16}$ are shown in Figure 2.11(a) and where they are compared against direct d-PMMT calculations. The d-PMMT model underpredicts these experimentally derived dissociative sticking coefficients $(A R D=$ 4911\%), particularly at the lowest translational energies where the signal-to-noise ratio and 
subtraction of background gas contribution in the $P\left(E_{t}, \vartheta=0^{\circ} ; v, J, T_{s}\right)$ distributions become increasing concerns in experimental determination of $S\left(E_{t}, \vartheta=0^{\circ} ; v, J, T_{s}\right)$.

Effective activations energies $" E_{a}\left(T_{s}\right) "=-k_{B} \partial \ln S / \partial T_{s}^{-1}$, calculated from the slopes of the best fit lines in the Arrhenius plots of Fig. 2.11(a), are plotted as a function of normal translational energy in Figure 2.11(b). Interestingly, the effective activation energies fall as the normal translational energy increases. The experimentally determined " $E_{a}\left(T_{s}\right)$ " fall linearly with constant slope $\partial " E_{a}\left(T_{s}\right) " / \partial E_{n}=-0.9$. The d-PMMT predicts $\partial " E_{a}\left(T_{s}\right) " / \partial E_{n}=-\eta_{s}^{-1}=-1.7$ initially and a falling off of the slope towards zero in the neighborhood of $E_{n}=E_{0}$. This theoretical behavior can be explained fairly readily. The non-equilibrium Tolman expression for the effective activation energy ${ }^{57}$ is $" E_{a}\left(T_{s}\right) "=-k_{B} \partial \ln S / \partial T_{s}^{-1}=\left\langle E_{s}\right\rangle_{R}-\left\langle E_{s}\right\rangle$, where $\left\langle E_{s}\right\rangle_{R}$ and $\left\langle E_{s}\right\rangle$ are the mean surface energies for those PCs that successfully react and for all the PCs formed, respectively. Furthermore, in order for a PC to successfully react the active exchangeable energy must be greater than the reaction threshold energy such that for eigenstate-resolved dissociative sticking experiments, $\left\langle E_{s}\right\rangle_{R} \geq\left[E_{o}-\left(E_{n}+\eta_{v} E_{v}+\eta_{r} E_{r}\right)\right] / \eta_{s}$. When molecular energy alone is insufficient to surmount the reaction barrier and $\left\langle E_{s}\right\rangle_{R}$ must be much greater than $\left\langle E_{s}\right\rangle$ to allow for reaction, the last expression effectively becomes an equality plus a $T_{s}-$ dependent constant and $\partial " E_{a}\left(T_{s}\right) " / \partial E_{n}=\partial\left\langle E_{s}\right\rangle_{R} / \partial E_{n}=-\eta_{s}^{-1}$. With increasing molecular energy (increasing $E_{n}$ in these experiments), the inequality constraint on $\left\langle E_{s}\right\rangle_{R}$ eventually becomes negligible such that $\left\langle E_{s}\right\rangle_{R} \rightarrow\left\langle E_{s}\right\rangle, " E_{a}\left(T_{s}\right) " \rightarrow 0$, and $\partial " E_{a}\left(T_{s}\right) " / \partial E_{n} \rightarrow 0$. 
2.3.D. Non-equilibrium Dissociative Sticking: Supersonic Molecular Beams with Surface at $T_{s}=120 \mathrm{~K}$.

Experimental dissociative sticking coefficients ${ }^{6}$ performed using a seeded supersonic beam of $\mathrm{H}_{2}$ with a heated nozzle impinging on $\mathrm{Cu}(111)$ surface at $T_{s}=120 \mathrm{~K}$ beam are reported as a function of normal translational energy in Fig. 2.12 along with predictions of the d-PMMT model and those from $6 \mathrm{D}$ quantum dynamics ${ }^{31,32}$ calculations. Both theoretical models make similar predictions for these experiments and the ARD with respect to experiments for the dPMMT model is $50 \%$. Unfortunately, experimental beam velocity and temperature parameters required for theoretical simulations are only available in the literature ${ }^{32}$ for this subset of an extended range of supersonic beam measurements made by Rettner, Michelsen, and Auerbach (RMA). ${ }^{6}$ The experimentalists found they could reproduce all their supersonic beam experimental results for the $T_{s}=120 \mathrm{~K}$ surface adequately well using detailed balance and the parameters derived from erf curve fits to state-resolved associative desorption experiments made at $T_{s}=925$ $\mathrm{K}$, given some assumptions about the surface temperature dependence of the erf parameters for $\mathrm{v}$ $=0,1$, free fitting of erf parameters for $\mathrm{v}=2$ to the $T_{s}=120 \mathrm{~K}$ experiments, and normalization of all the state-resolved dissociative sticking coefficients to a common limiting high value of $0.25 .^{6}$

\subsection{E. Thermal Dissociative Sticking: Ambient Gas}

In Fig. 2.13(a) the thermal dissociative sticking coefficient, $S(T)$, for ambient $\mathrm{H}_{2}$ gas above an isothermal $\mathrm{Cu}(111)$ surface calculated by the d-PMMT model is compared to RMA's extrapolation $^{6}$ based on their Erf analysis of thermal associative desorption at $T_{s}=925 \mathrm{~K}$, and the non-equilibrium dissociative sticking of supersonic molecular beams at $T_{s}=120 \mathrm{~K}$ discussed above. Also shown for comparison are direct experimental measurements of $S(T)$ for the 
$\mathrm{H}_{2} / \mathrm{Cu}(110)$ system $^{58}$ because there have been no modern surface science measurements of $S(T)$ for $\mathrm{H}_{2} / \mathrm{Cu}(111){ }^{59}$ Agreement across these estimates for the $\mathrm{H}_{2} / \mathrm{Cu}(111) S(T)$ is reasonably good. Over the $300 \mathrm{~K} \leq T \leq 1000 \mathrm{~K}$ temperature range, the best Arrhenius fit to the d-PMMT model calculations is $S(T)=S_{0} \exp \left(-E_{a} / R T\right)$ where $S_{0}=0.075$ and $E_{a}=49.2 \mathrm{~kJ} / \mathrm{mol}$. The activation energy at $925 \mathrm{~K}$ according to the RMA extrapolation ${ }^{6}$ is $48.4 \mathrm{~kJ} / \mathrm{mol}$. To assess the impact of dynamics on $S(T)$ an additional calculation was made for a statistical(s) -PMMT model in which all the efficacies of the d-PMMT model were set to their limiting statistical values of one (i.e., $\eta_{i} \rightarrow 1$ ). Fig. 2.13(a) indicates that over the temperature range of catalytic interest, dynamical effects are substantial and serve to reduce the thermal gas-surface reactivity by two orders of magnitude as compared to statistical expectations.

Figure 2.13(b) extends d-PMMT calculations of $S(T)$ for $\mathrm{H}_{2}$ and $\mathrm{D}_{2}$ to lower temperatures where tunneling through the reaction barrier is signaled by exaggerated curvature and a kneeing of the Arrhenius plots. To make the effects of tunneling explicitly apparent, dPMMT calculations of $S(T)$ without inclusion of tunneling pathways are also shown. Tunneling clearly dominates the reactivity at the lowest temperatures where it can increase the tunnelingexcluded reactivity by many orders of magnitude. Interestingly, even at the high temperatures of catalytic interest, tunneling pathways remain responsible for a significant fraction of the observed reactivity. The d-PMMT calculations of Fig. 2.14 indicate that tunneling pathways account for $13 \%$ of $S(T)$ at $1000 \mathrm{~K}$, increasing to greater than $50 \%$ as the temperature falls below $400 \mathrm{~K}$.

Fractional (exchangeable) energy uptakes defined as $f_{i}=\left\langle\eta_{i} E_{i}\right\rangle_{R} /\left\langle E^{*}\right\rangle_{R}$, where $\left\langle\eta_{i} E_{i}\right\rangle_{R}$ is the mean exchangeable energy derived from the $i^{\text {th }}$ degree of freedom for the successfully reacting PCs formed and $\left\langle E^{*}\right\rangle_{R}$ is the mean exchangeable energy for the 
successfully reacting PCs formed, respectively, are useful for assessment of which degrees of freedom are most important in supplying the energy required to surmount the activation barrier to dissociative chemisorption. ${ }^{38}$ Fig. 2.15 reports fractional energy uptakes calculated for thermal dissociative chemisorption over a range of temperatures of catalytic interest, and also as a function of molecular angle of incidence for reaction at $1000 \mathrm{~K}$. The angle-integrated $f_{i}$ of Fig. 2.15 (a) show that normal translational energy typically supplies more than $65 \%$ of the energy required to surmount the activation barrier, the surface contributes $10-20 \%$, rotation about $5 \%$, and vibration less still. The angle-resolved $f_{i}$ of Fig. 2.15(b) for reaction at $1000 \mathrm{~K}$ show that when normal translational energy $\left(E_{n}=E_{t} \cos ^{2} \vartheta\right)$ is readily available near $\vartheta=0^{\circ} f_{t}$ is particularly high but as $\vartheta$ increases and $E_{n}$ falls off then it is increasingly energy from the surface and molecular vibration that is used to surmount the activation barrier. Indeed, for $\vartheta>60^{\circ}$ the preponderance of the energy used to surmount the activation barrier derives from the surface. An important point to note from Fig. 2.15 is that surface vibrational energy (phonons) always plays a substantial role in the activated thermal dissociative chemisorption, and, apparently, a more substantial role than that for molecular internal energy.

Given that the fractional energy uptake from rotation is always small and relatively constant at $f_{r} \sim 5 \%$ in Fig. 2.15 , it may be reasonable to simply treat rotation as a spectator degree of freedom. d-PMMT simulations performed with rotation as a spectator Figure 2.16-2.19 were found capable of reproducing the majority of the experimental data available with comparable ARDs to those demonstrated here. In that case, optimal parameters for $\mathrm{H}_{2} / \mathrm{Cu}(111)$ were $\left\{E_{0}=\right.$ $\left.49.2 \mathrm{~kJ} / \mathrm{mol}, s=1, \eta_{v}=0.50, \eta_{s}=0.60\right\}$. The finding that rotation plays a relatively insignificant role in the activated dissociative chemisorption of this benchmark $\mathrm{H}_{2} / \mathrm{Cu}(111)$ system with fully state-resolved experimental data is interesting because the same rotation as a 
spectator approximation has also met with some success in simulations of the dissociative chemisorption of larger molecules, such as $\mathrm{CO}_{2} / \mathrm{Rh}(111)^{60}$ and $\mathrm{CH}_{4} / \mathrm{Pt}(111) .{ }^{38}$ If rotation could generally be approximated as a spectator degree of freedom in activated dissociative chemisorption it would simplify modeling of these important gas-surface reactions.

\subsection{F. Comparison to Other Work}

\subsection{F.I. Analysis with Error Function Sticking Model}

Rettner and coworkers ${ }^{6,7}$ and also Murphy and Hodgson ${ }^{16}$ modeled their state-resolved associative desorption and molecular beam dissociative sticking experiments for hydrogen on $\mathrm{Cu}(111)$ by assuming the molecular eigenstate-resolved dissociative sticking coefficient obeyed a semi-empirically defined error function "Erf" form,

$$
S\left(E_{t}, \vartheta ; v, J, T_{s}\right)=\frac{A(v, J)}{2}\left[1+E r f\left(\frac{E_{e}-\bar{E}_{d}(v, J)}{W\left(v, J ; T_{s}\right)}\right)\right]
$$

in which $A(v, J), \bar{E}_{d}(v, J)$, and $W\left(v, J ; T_{s}\right)$ are adjustable parameters. An effective translational energy is defined as,

$$
E_{e}=E_{t} \cos ^{n} \vartheta
$$

where $n$ is an adjustable parameter. Sticking coefficients of the Eq. (2.17) form have a sigmoid shape as a function of $E_{e}$. Rettner and coworkers assumed normal translational energy scaling of $S$ with $n=2$ was applicable, ${ }^{6}$ but Murphy and Hodgson ${ }^{16}$ argued that $n=1.8$ or $n=n\left(E_{t}\right)$ could improve agreement with experiments. The $A(v, J)$ parameter is the limiting value of the sticking coefficient at high $E_{e}, \bar{E}_{d}(v, J)$ is the value of $E_{e}$ when the sticking has reached half its limiting value, and $W\left(v, J ; T_{s}\right)$ is a width parameter. There is sufficient flexibility in the Erf functionality 
that state-resolved experimental data can be fit very well. The success of the Erf functionality is typically ascribed to the idea that a Gaussian distribution of reaction barriers is sampled by the ensemble of incident molecules colliding with the surface. ${ }^{13,61-63}$ The particular barrier an individual molecule encounters is presumed to be a function of the molecule's state, and its experimentally-uncontrolled orientation, vibrational phase, and impact parameter across the surface unit cell. Under the premises of the Erf model, $\bar{E}_{d}(v, J)$ is the mean dynamical barrier to reaction encountered for a molecule incident in the $(v, J)$ rovibrational state.

Rettner and coworkers ${ }^{6,7}$ experimentally determined a vibrational efficacy for reaction defined as,

$$
\eta_{v, \mathrm{e}}=\frac{\bar{E}_{d}(v-1, J)-\bar{E}_{d}(v, J)}{E_{v}(v-1, J)-E_{v}(v, J)}=\frac{\Delta \bar{E}_{d}}{\Delta E_{v}}
$$

to characterize how efficacious vibrational energy was in lowering the translational energy requirement to surmount the mean dynamical barrier [c.f., Eqs (2.6-2.7)]. The experimentally derived $\bar{E}_{d}(v, J)$ values are numerically very similar to the rovibrationally resolved $\left\langle E_{t}\right\rangle$ values of Fig. 2.4. For $\mathrm{H}_{2}$ and $\mathrm{D}_{2}$ on $\mathrm{Cu}(111),{ }^{6}$ experiments found that $\eta_{v, e}=0.51 \pm 0.02$ for $v=1$ and 2, independent of $J$ state and isotope. The rotational efficacy, $\eta_{r, e}$, defined similarly to Eq. (2.19), was initially negative, beginning near -1 , because $\bar{E}_{d}(v, J)$ initially increases slightly with $J$ (c.f., $\left\langle E_{t}\right\rangle$ in Fig. 2.4). With increasing $J$, the rotational efficacy steadily increases before leveling off to a constant value within the range $0.25-0.48$, depending weakly on vibrational state and isotope. Rettner and coworkers' efficacy findings ${ }^{6,7}$ based on Erf model analysis of their experiments are essentially recapitulated by the d-PMMT model analysis which finds $\eta_{v}=0.60$ and $\eta_{r}=-0.45+0.78 \operatorname{Erf}\left[0.026 E_{r}\right]$, independent of vibrational state and 
isotope, such that $\eta_{r}$ varies from -0.45 to a limiting value of 0.33 as $J$ increases. However, the d-PMMT model extracts an additional efficacy value for surface vibrational energy of $\eta_{s}=0.60$. The Erf model does not allow for calculation of a surface efficacy but does find that the width parameter $W\left(v, J ; T_{s}\right)$, which determines the broadness of the transition region of the sigmoid shaped sticking curve [i.e., $S\left(E_{t}, \vartheta ; v, J, T_{s}\right)$ is sigmoidal when plotted on a linear scale versus $E_{e}$ ], increases with $T_{s}$.

There is some economy of parameterization gained when fitting the experimental data to the d-PMMT model as compared to the Erf model. Seven d-PMMT parameters were fixed by fitting the experimental state-resolved associative desorption data of Figs 2.3-2.4 alone. Subsequently, a range of other desorption phenomena and dissociative sticking coefficients at different surface temperatures could be at least semi-quantitatively reproduced (i.e., Figs 2.62.13). The Erf model requires fixing 3 parameters per quantum state at a given surface temperature and so to optimally describe the sticking of the $19 \mathrm{H}_{2}$ and $36 \mathrm{D}_{2}$ rovibrationally resolved states of Fig. 2.4 requires specification of 165 parameters. Additional dissociative sticking coefficients experiments are required to fix absolute values for the $A(v, J)$ and some experiments at different temperatures are needed to fix further parameters to describe the surface temperature dependence of the $W\left(v, J ; T_{s}\right)$ parameters.

The d-PMMT model can be considered in the context of an information theory/maximal entropy formalism ${ }^{64,65}$ to be one for which the prior distribution is a s-PMMT (maximal entropy) model and the efficacy values constitute the dynamical constraints necessary to recover the experimental results. To the extent that the 5 efficacy parameters of the d-PMMT model allow for adequate reproduction of the experimental data, the dynamical information content of the experiments can be summarized by 5 or less independent parameters. Outside these dynamical 
constraints, the $\mathrm{H}_{2}\left(\mathrm{D}_{2}\right) / \mathrm{Cu}(111)$ system apparently behaves according to the precepts of conventional microcanonical transition state theory. By comparison, it seems that the Erf model is over-parameterized and there is no compelling necessity to invoke $\left(v, J, T_{s}\right)$ state-specific Gaussian distributions of dynamical barriers to explain the variation of $S\left(E_{t}, \vartheta ; v, J, T_{s}\right)$ with $E_{t}$, despite its fairly wide-spread practice within the gas-surface reaction dynamics community.

\subsection{F.II. Dynamical Simulations}

Dynamical simulations of increasing dimensionality ${ }^{32,53,66}$ have been evolving to treat the $\mathrm{H}_{2} / \mathrm{Cu}(111)$ dissociative chemisorption. Most recently, Kroes and coworkers ${ }^{31-33}$ used sixdimensional (6D) dynamical calculations, addressing all molecular degrees of freedom but assuming a static $\mathrm{Cu}$ surface, to optimize the specific reaction parameter of a SRP-DFT PES for $\mathrm{H}_{2} / \mathrm{Cu}(111)$ such that some experimental data (e.g., Fig. 2.12 and another similar to Fig. 2.4) could be reproduced sufficiently well to claim chemical accuracy (to within $4.2 \mathrm{~kJ} / \mathrm{mol}$ ) for the SRP-DFT PES. The threshold energy for dissociative chemisorption of $\mathrm{H}_{2} / \mathrm{Cu}(111)$ for the SRPDFT PES is $E_{0}=56.5 \mathrm{~kJ} / \mathrm{mol}$, somewhat higher than the $E_{0}=43.4 \mathrm{~kJ} / \mathrm{mol}$ and $E_{a}=49.2 \mathrm{~kJ} / \mathrm{mol}$ values of the d-PMMT model, or the RMA estimation of the thermal activation energy at $925 \mathrm{~K}$ of $E_{a}=48.4 \mathrm{~kJ} / \mathrm{mol}$. On the other hand, higher dimensional dynamical calculations including surface degrees of freedom might lead to a lower $E_{0}$ in a SRP-DFT PES optimization procedure because new non-reactive energy transfer processes involving the surface might more successfully compete with new reactive channels such that $E_{0}$ would need to be lowered to match the experimental reactivity. Certainly, Hodgson's experimental data and analysis (Figs. $2.10 \& 2.11$ ) dramatically demonstrate that surface energy can play a significant role in the gassurface reaction dynamics. The d-PMMT model predicts fractional energy uptakes of $f_{t}=75.4 \%$, 
$f_{s}=17 \%, f_{r}=4.3 \%$, and $f_{v}=3.3 \%$ (Fig. 15) for the thermal dissociative chemisorption at $925 \mathrm{~K}$ that is relevant to the current SRP-DFT PES optimization based, at least in part, on 6D dynamical calculations reproducing state-resolved $\bar{E}_{d}(v, J)$ [similar to the $\left\langle E_{t}(v, J)\right\rangle$ of Fig. 2.4] derived from $925 \mathrm{~K}$ thermal associative desorption experiments. The predicted fractional energy uptake from surface phonons, $f_{s}=17 \%$, in the thermal dissociative chemisorption seems too large to ignore and should encourage the development of higher dimensional dynamical calculations for the $\mathrm{H}_{2} / \mathrm{Cu}(111)$ reactive system.

\subsection{Summary}

A minimally parameterized, d-PMMT model of the dissociative chemisorption of hydrogen on $\mathrm{Cu}(111)$ was developed and shown capable of, at least semi-quantitatively, reproducing the diverse range of experimental results available for this benchmark system for gas-surface reaction dynamics. At temperatures of catalytic interest, dynamical biases serve to reduce the $\mathrm{H}_{2} / \mathrm{Cu}(111)$ thermal reactivity by several orders of magnitude as compared to the expectations of statistical transition state theory. The d-PMMT model indicated that although the energy necessary to surmount the activation barrier in thermal dissociative chemisorption was supplied primarily by molecular translational energy directed along the surface normal, second in importance was surface vibrational energy which was vital to include in the theoretical modeling. Tunneling was calculated to contribute only modestly to the thermal reactivity at temperatures of catalytic interest. 


\section{Tables}

\begin{tabular}{|c|c|c|c|c|c|}
\hline & $\mathrm{v}$ & $\mathrm{J}$ sum & $\begin{array}{l}\mathrm{P}_{\mathrm{v}}-\text { Expt. }^{6,7} \\
|\vartheta| \leq 20^{\circ}\end{array}$ & $\begin{array}{l}\mathrm{P}_{\mathrm{v}}-\mathrm{d}-\mathrm{PMMT} \\
|\vartheta| \leq 20^{\circ}\end{array}$ & $\begin{array}{l}\mathrm{P}_{\mathrm{v}}-\mathrm{d}-\mathrm{PMMT} \\
\text { Angle integrated }\end{array}$ \\
\hline \multirow[t]{3}{*}{$\mathrm{H}_{2}$} & 0 & $0-10$ & $96.7 \%$ & $96 \%$ & $93.7 \%$ \\
\hline & 1 & $0-7$ & $3.3 \%$ & $3.9 \%$ & $6.1 \%$ \\
\hline & \multicolumn{2}{|c|}{$\sum \mathrm{P}_{\mathrm{v}, \mathrm{J}}$} & $100 \%$ & $99.9 \%$ & $99.8 \%$ \\
\hline \multirow[t]{4}{*}{$\overline{D_{2}}$} & 0 & $0-14$ & $82.4 \%$ & $90.2 \%$ & $86.3 \%$ \\
\hline & 1 & $0-11$ & $16.8 \%$ & $9.1 \%$ & $13.0 \%$ \\
\hline & 2 & $0-8$ & $0.8 \%$ & $0.6 \%$ & $0.7 \%$ \\
\hline & \multicolumn{2}{|c|}{$\Sigma \mathrm{P}_{\mathrm{v}, \mathrm{J}}$} & $100 \%$ & $99.9 \%$ & $100 \%$ \\
\hline
\end{tabular}

Table 2.1. Vibrational product state distributions for thermal associative desorption of $\mathrm{H}_{2}$ and $\mathrm{D}_{2}$ from $\mathrm{Cu}(111)$ at $925 \mathrm{~K}$. 
Figures

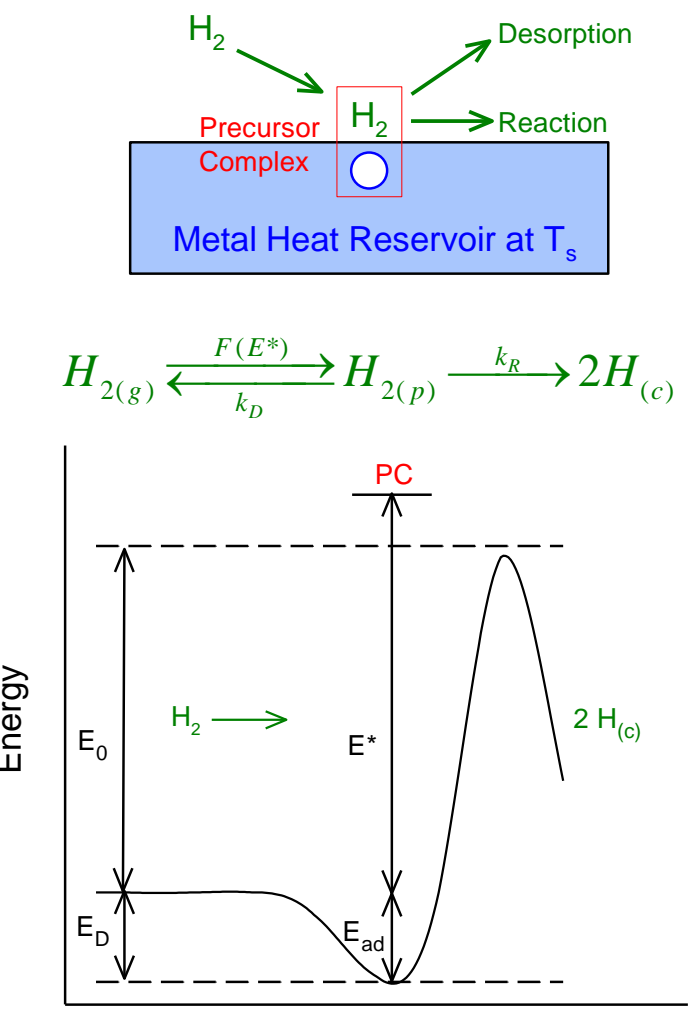

Reaction Coordinate

Figure 2.1: Schematic depiction of the kinetics and energetics of hydrogen dissociative chemisorption via precursor-mediated microcanonical trapping (PMMT). At energies sufficient to react, collisionally formed precursor complexes, PCs, comprised of a hydrogen molecule interacting with $s$ surface oscillators in the spatial vicinity of the physisorption well, are presumed to become transiently trapped between the transition states for desorption and reaction. Zero-point energies are implicitly included within the potential energy curve along the reaction coordinate. 

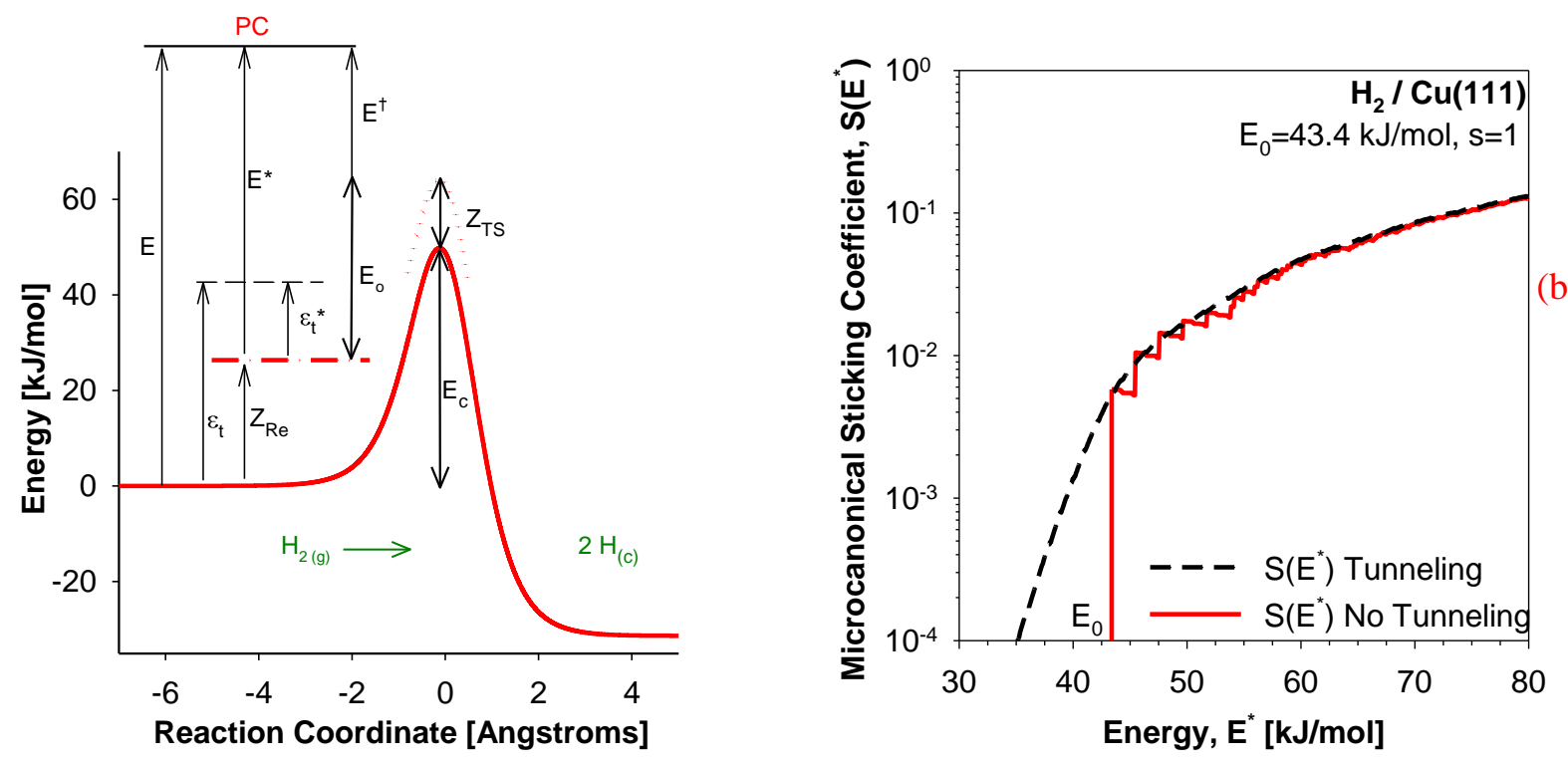

Figure 2.2: (a) The 1D Eckart potential representing the barrier for $\mathrm{H}_{2} / \mathrm{Cu}(111)$ dissociative chemisorption derived from the Kroes' 6D SRP-DFT-PES with the d-PMMT optimized threshold energy of $E_{0}=43.4 \mathrm{~kJ} / \mathrm{mol}$ (c.f., SRP-DFT-PES value of $E_{0}=56.5 \mathrm{~kJ} / \mathrm{mol}$ ). The zero-point energies of the separated reactants and the transition state, $Z_{R e}$ and $Z_{T S}$, as well as some other energies relevant to the RRKM and tunneling Eqs. (9-13) are also labeled. (b) Microcanonical dissociative sticking coefficient, $S\left(E^{*}\right)$, evaluated with and without tunneling. 


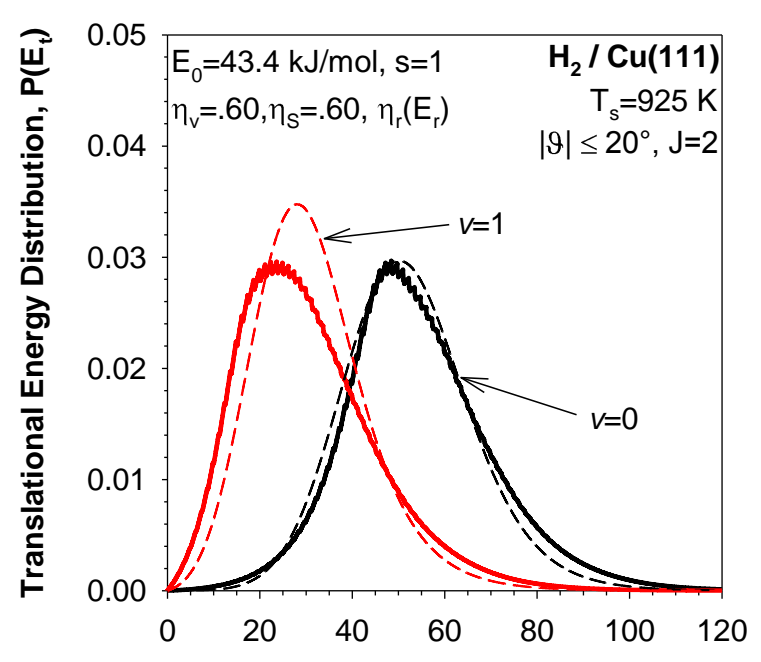

(a)

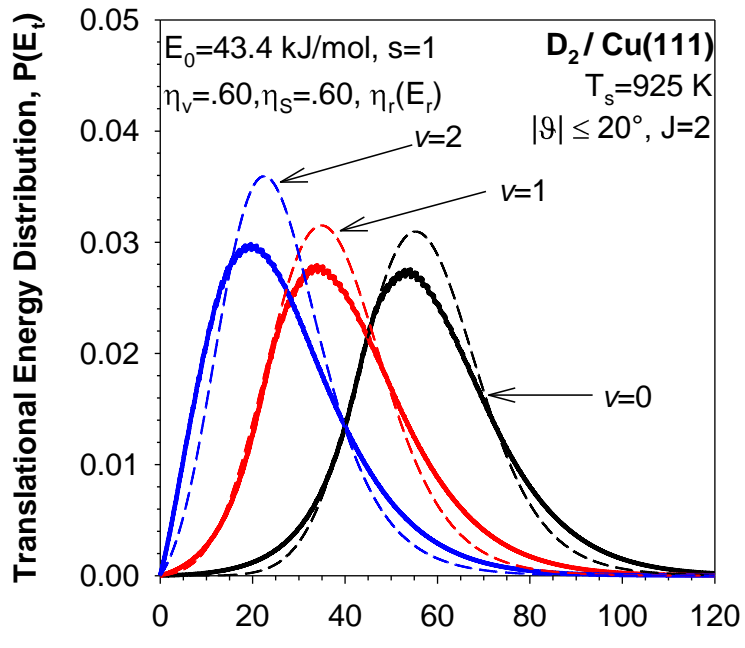

(b)

Translational Energy, $E_{t}[\mathrm{~kJ} / \mathrm{mol}]$

Figure 2.3: Vibrational state dependence of experimentally-derived translational energy distributions (dashed lines) ${ }^{6,7}$ for the associative desorption of (a) $\mathrm{H}_{2}(v, J=2)$ and (b) $\mathrm{D}_{2}(v$, $J=2$ ) are compared to d-PMMT simulations (lines). 


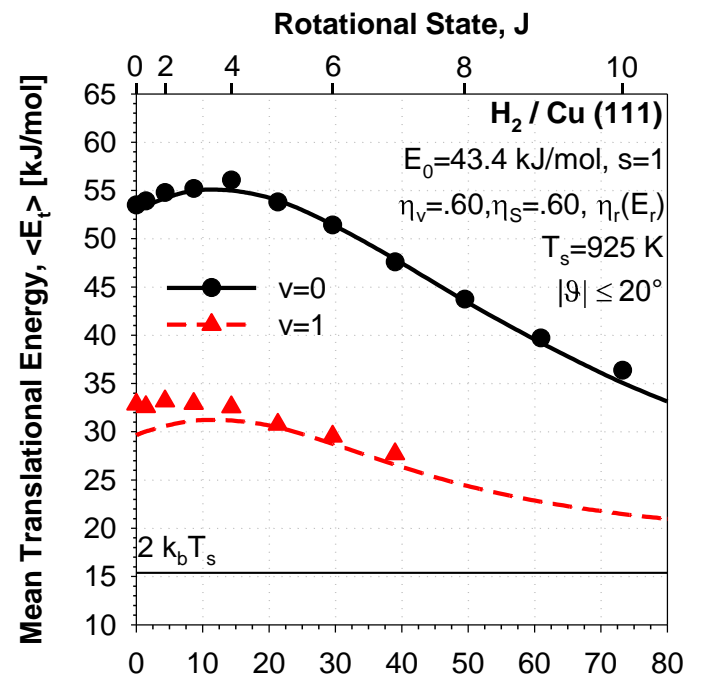

(a)

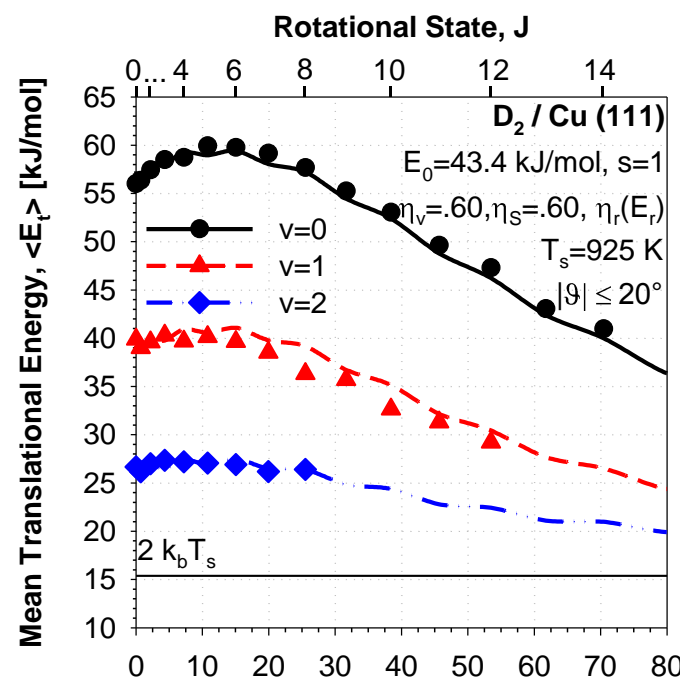

(b)

Rotational Energy, $E_{r}[\mathrm{~kJ} / \mathrm{mol}]$

Figure 2.4: Measured rovibrational state resolved mean translational energies (points) ${ }^{6,7}$ for recombinatively desorbing (a) $\mathrm{H}_{2}$ and (b) $\mathrm{D}_{2}$ are compared to d-PMMT simulations (lines). A reference line demarking the $\left\langle E_{t}\right\rangle=2 k_{B} T_{s}$ expectation for a system exhibiting a dissociative sticking coefficient independent of $E_{t}$ is also provided. 


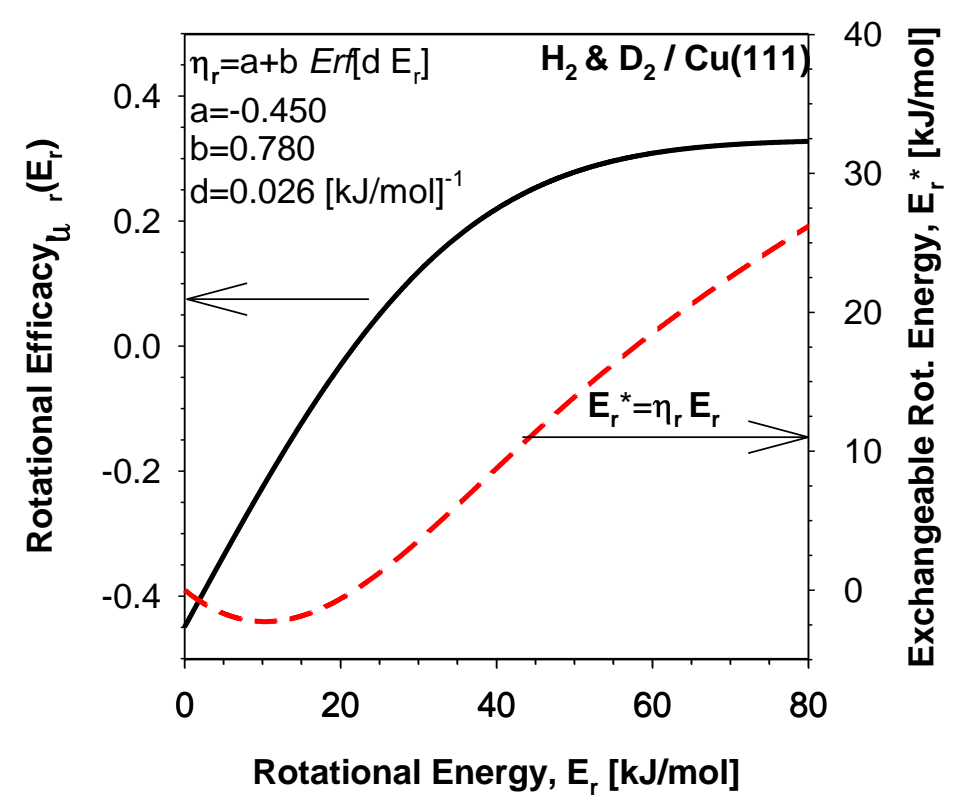

Figure 2.5: The efficacy of rotation (solid line) and exchangeable rotational energy (dashed line) used in d-PMMT simulations are plotted as a function of the rotational energy of the incident molecules for hydrogen dissociation on $\mathrm{Cu}(111)$. 


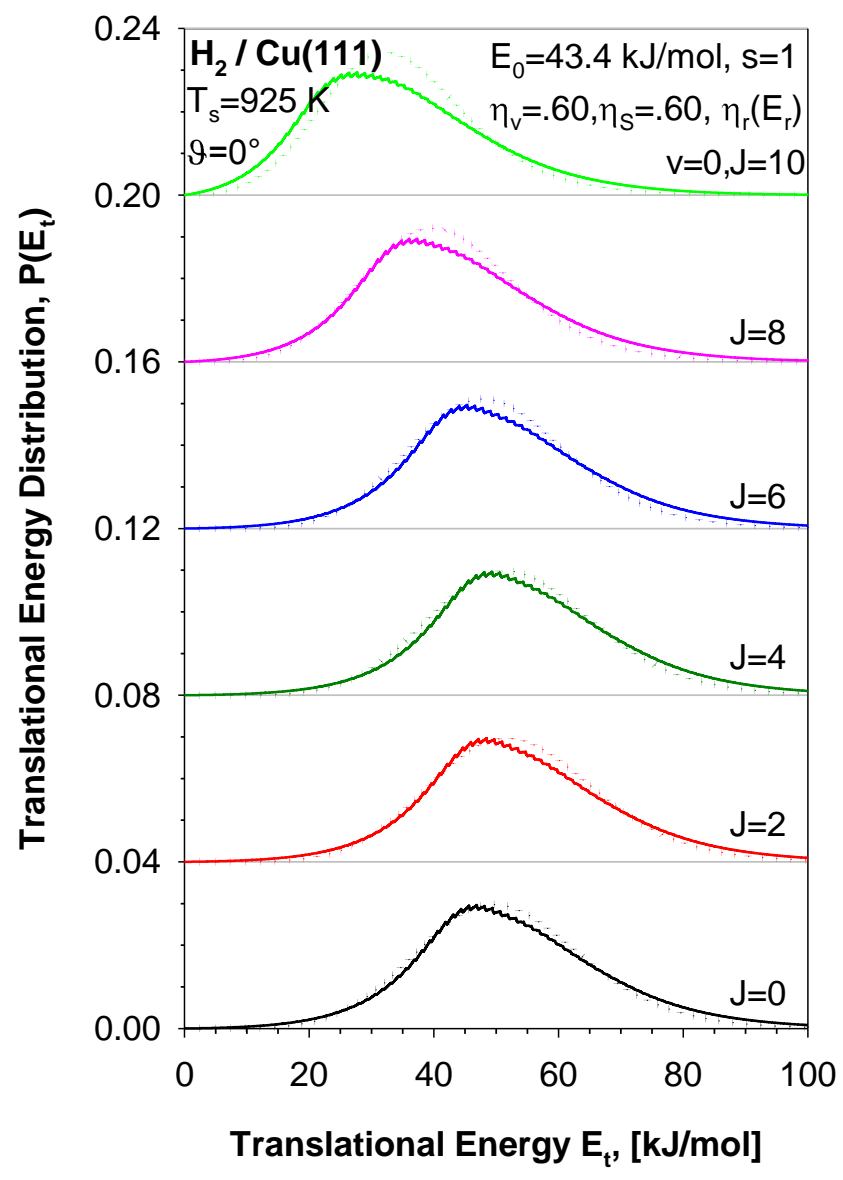

Figure 2.6: Rotational state dependence of measured translational energy distributions (points) ${ }^{6}$ for the associative desorption of $\mathrm{H}_{2}(v=0, J)$ are compared to d-PMMT simulations (lines). 

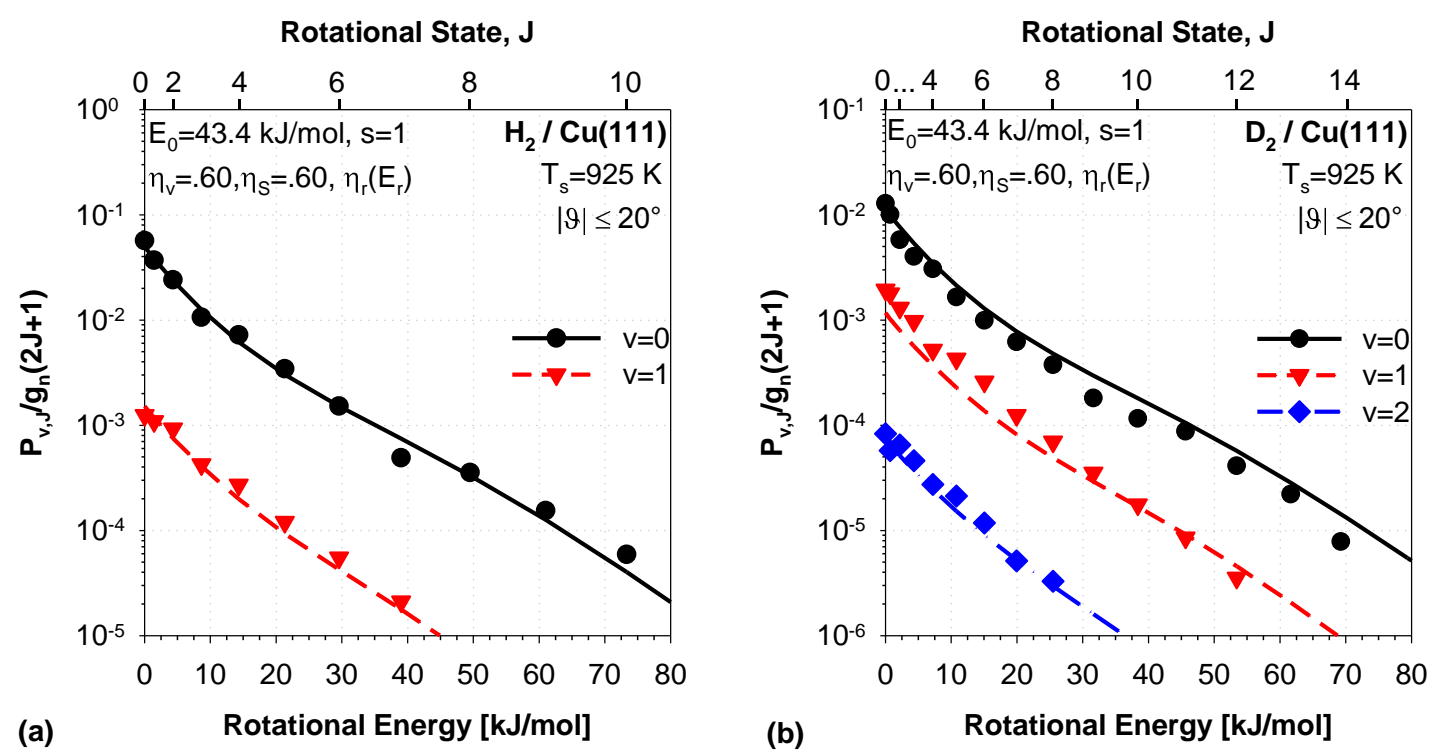

Figure 2.7: Boltzmann plots compare the measured vibrationally-resolved rotational energy distributions (points) ${ }^{6,7}$ for associative desorption of (a) $\mathrm{H}_{2}$ and (b) $\mathrm{D}_{2}$ to d-PMMT simulations (lines). 


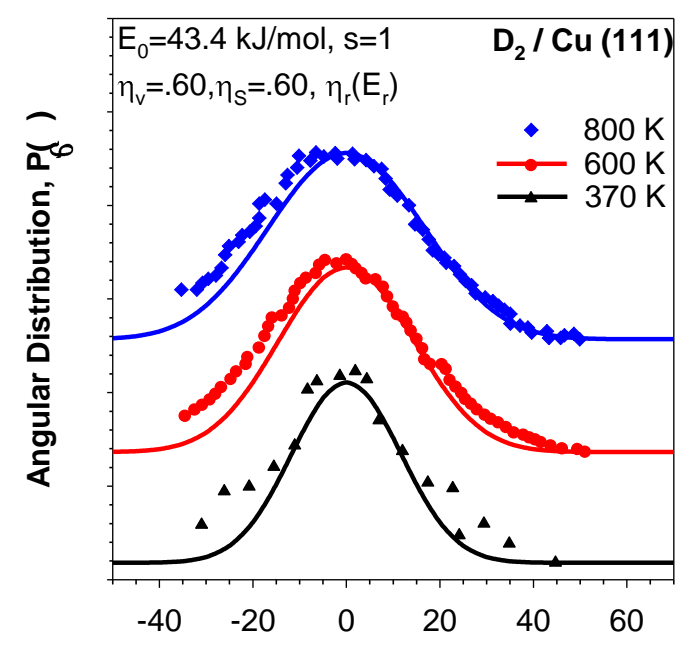

(a)

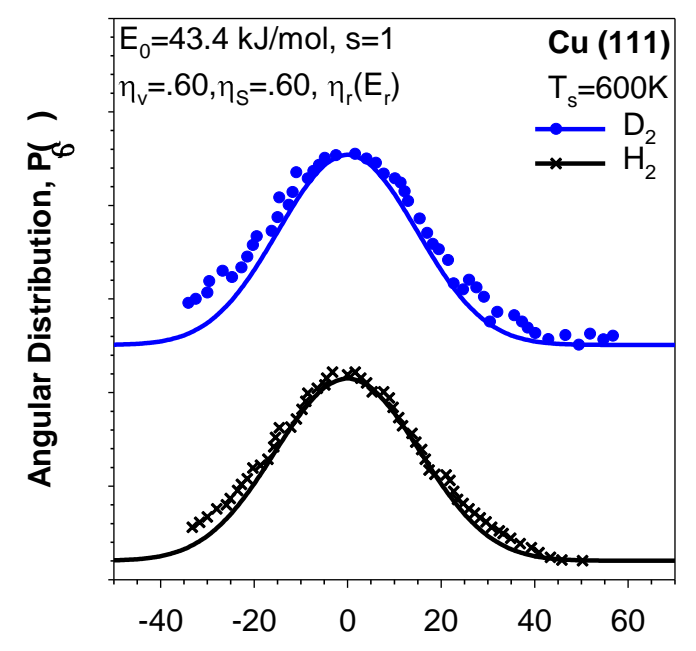

(b)

Desorption Angle, $\vartheta$ [Degree]

Figure 2.8: Measured angular distributions (points) ${ }^{8}$ for $\mathrm{H}_{2}$ and $\mathrm{D}_{2}$ recombinative desorption from $\mathrm{Cu}(111)$ at several surface temperatures are compared to d-PMMT simulations (lines). 


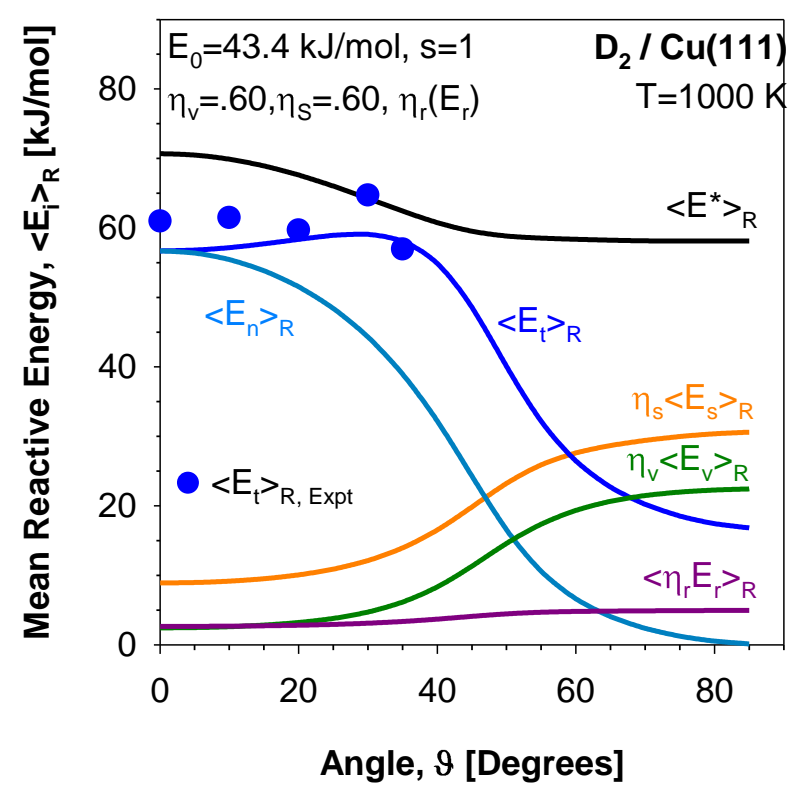

Figure 2.9: Measured mean translational energies (points) ${ }^{54}$ for the fast component of $D_{2}$ recombinative desorption as a function of angle are compared to d-PMMT predictions (lines) of the mean energies derived from different degrees of freedom for the successfully reacting precursor complexes formed in thermal dissociative chemisorption of $\mathrm{D}_{2}$ at $1000 \mathrm{~K}$. 

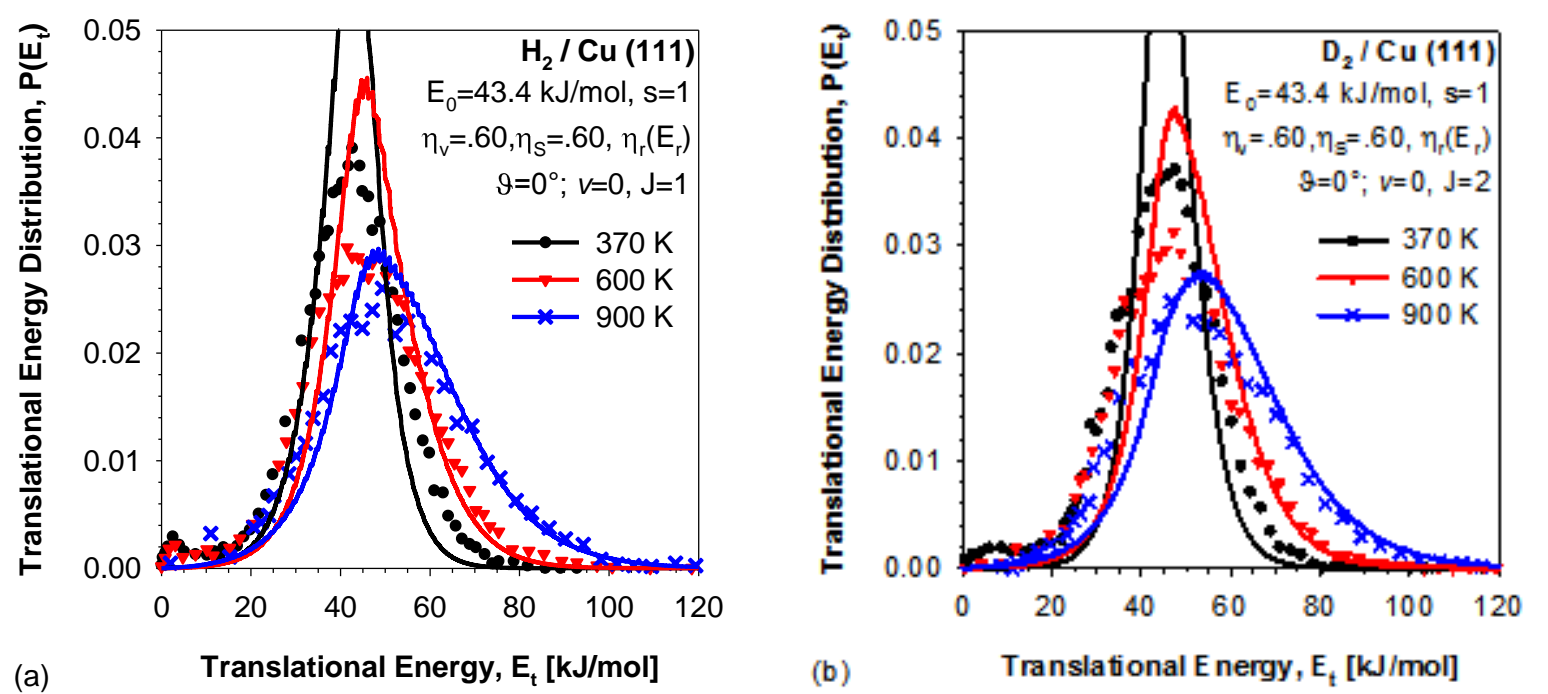

Figure 2.10: Measured rovibrational state resolved translational energy distributions (points) ${ }^{16}$ for the associative desorption of (a) $\mathrm{H}_{2}(v, J=1)$ and (b) $\mathrm{D}_{2}(v, J=2)$ at several surface temperatures are compared to d-PMMT simulations (lines). 

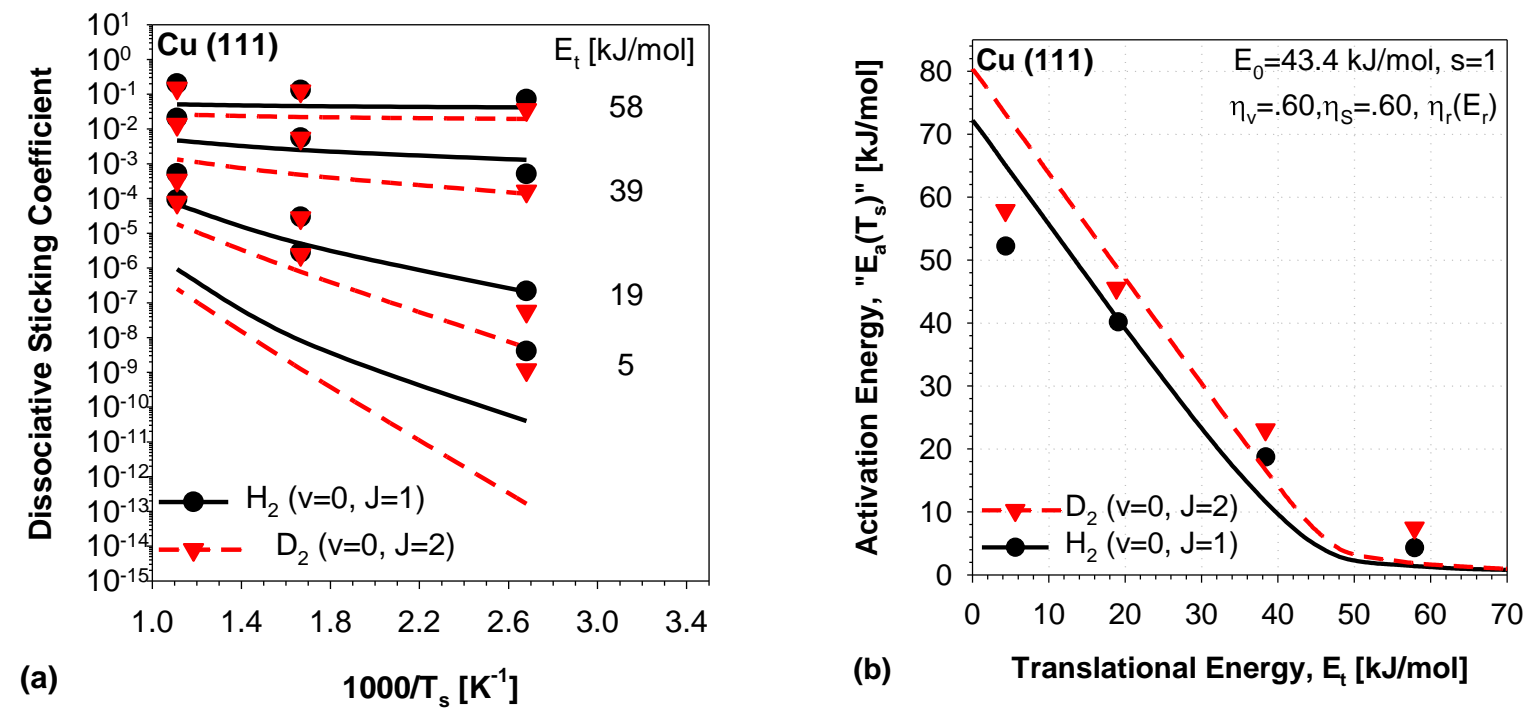

Figure 2.11: (a) Surface temperature dependent, rovibrational state resolved, relative dissociative sticking coefficients (points) ${ }^{16}$ derived from the measured associative desorption energy distributions of Fig. 2.9 are compared to d-PMMT simulations of absolute dissociative sticking coefficients (lines). (b) Effective activation energies derived from Arrhenius plots of the dissociative sticking coefficients shown in (a) are plotted for experiments (solid points) and dPMMT simulations (lines). 


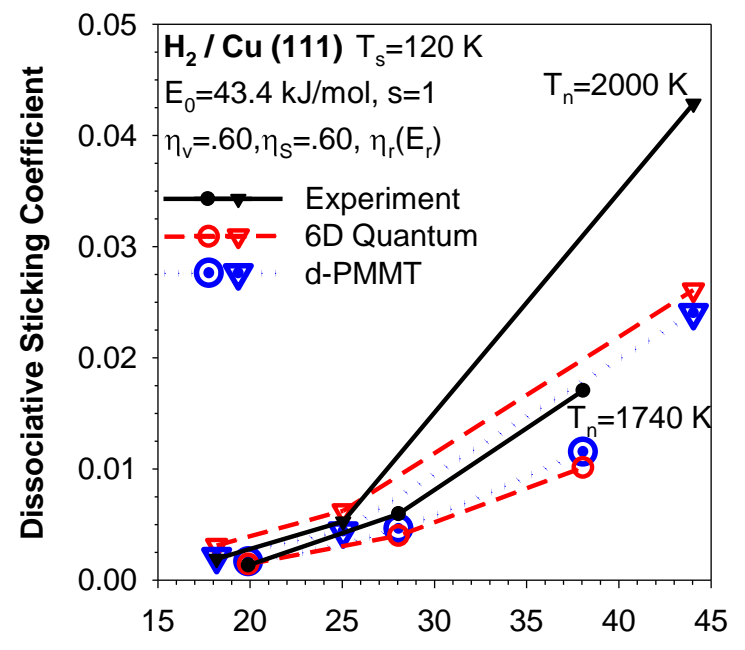

Normal Translational Energy, $E_{n}[\mathrm{~kJ} / \mathrm{mol}]$

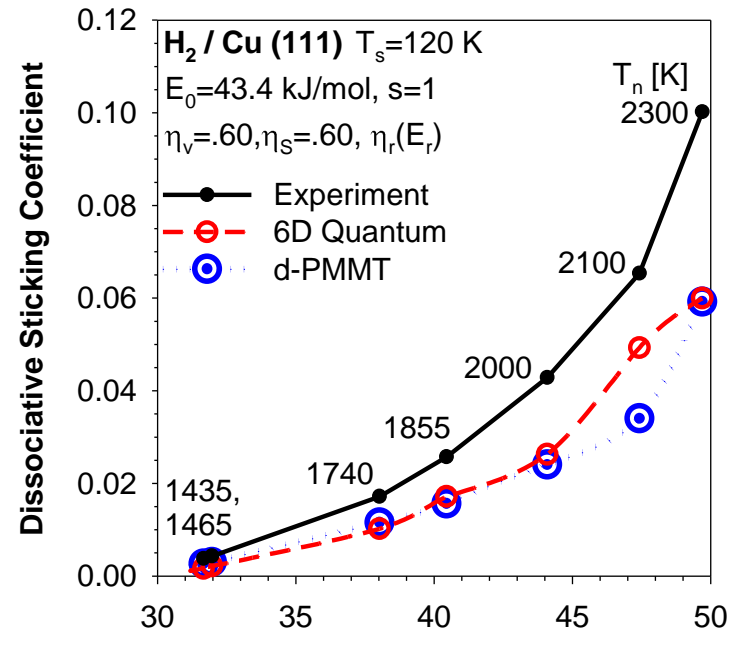

Normal Translational Energy, $E_{n}[\mathrm{~kJ} / \mathrm{mol}]$

Figure 2.12: Non-equilibrium dissociative sticking coefficients for supersonic molecular beams of hydrogen impinging on $\mathrm{Cu}(111)$ (filled points) ${ }^{6}$ are compared to $6 \mathrm{D}$ quantum dynamics ${ }^{31,32}$ and d-PMMT simulations (open points). 
$\mathrm{T}[\mathrm{K}]$

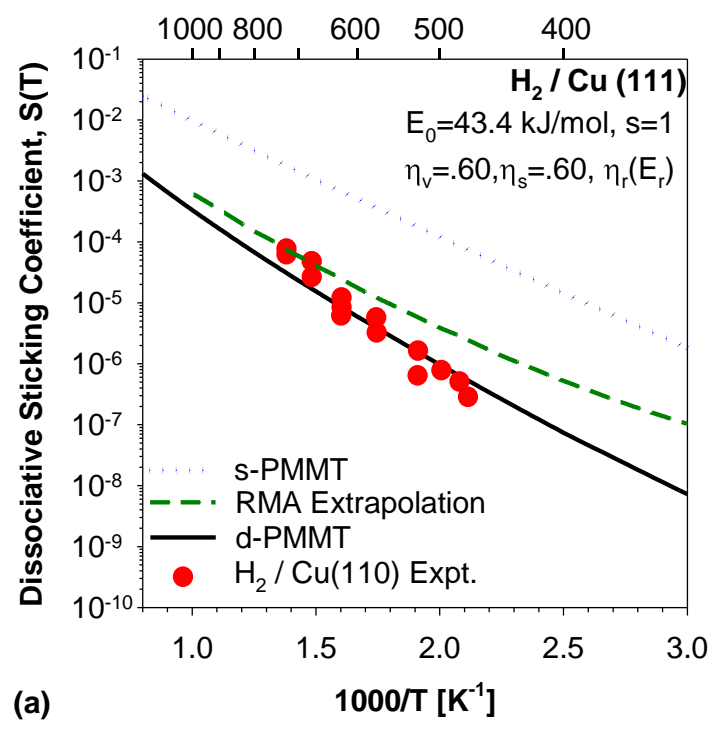

$\mathbf{T}[\mathrm{K}]$

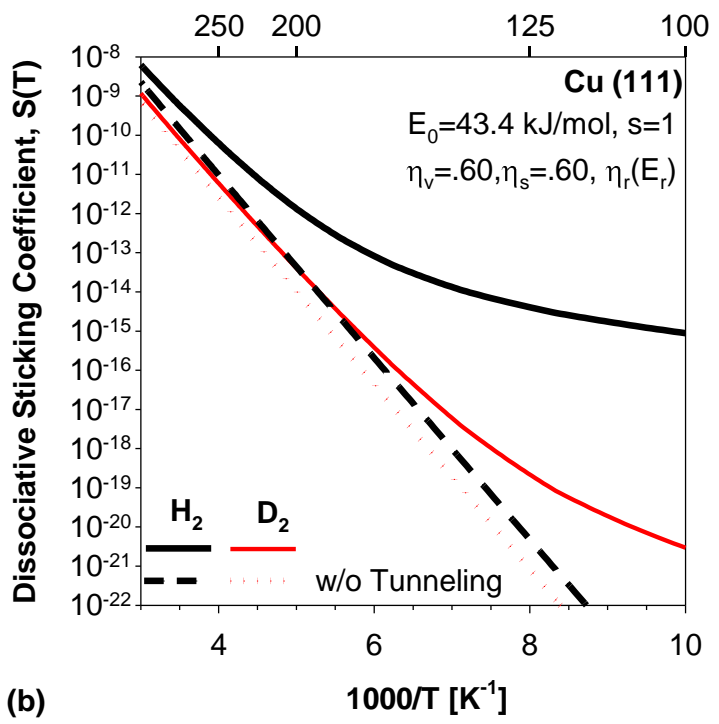

$\begin{array}{lll}\mathrm{Cu}(111) \mathrm{d}-\mathrm{PMMT}\left(\mathrm{H}_{2}\right): & \mathrm{Cu}(111) \mathrm{RMA}\left(\mathrm{H}_{2}\right): & \mathrm{Cu}(110) \text { Experiment: } \\ \mathrm{E}_{\mathrm{a}}=49.2 \mathrm{~kJ} / \mathrm{mol} & \mathrm{E}_{\mathrm{a}}=37.6 \mathrm{~kJ} / \mathrm{mol} & \mathrm{E}_{\mathrm{a}}=59.6 \mathrm{~kJ} / \mathrm{mol} \\ \mathrm{S}_{0}=.075 & \mathrm{~S}_{0}=.04 & \mathrm{~S}_{0}=1.10944\end{array}$

Figure 2.13: (a) Thermal dissociative sticking coefficient for $\mathrm{H}_{2} / \mathrm{Cu}(111)$ calculated by several models and experimental values ${ }^{58}$ for $\mathrm{H}_{2} / \mathrm{Cu}(100)$. (b) d-PMMT thermal dissociative sticking coefficients for $\mathrm{H}_{2}$ and $\mathrm{D}_{2}$ on $\mathrm{Cu}(111)$ are compared at lower temperatures where the effects of tunneling through the reaction barrier can be discerned (solid lines). Dashed lines give equivalent d-PMMT calculations without tunneling. 


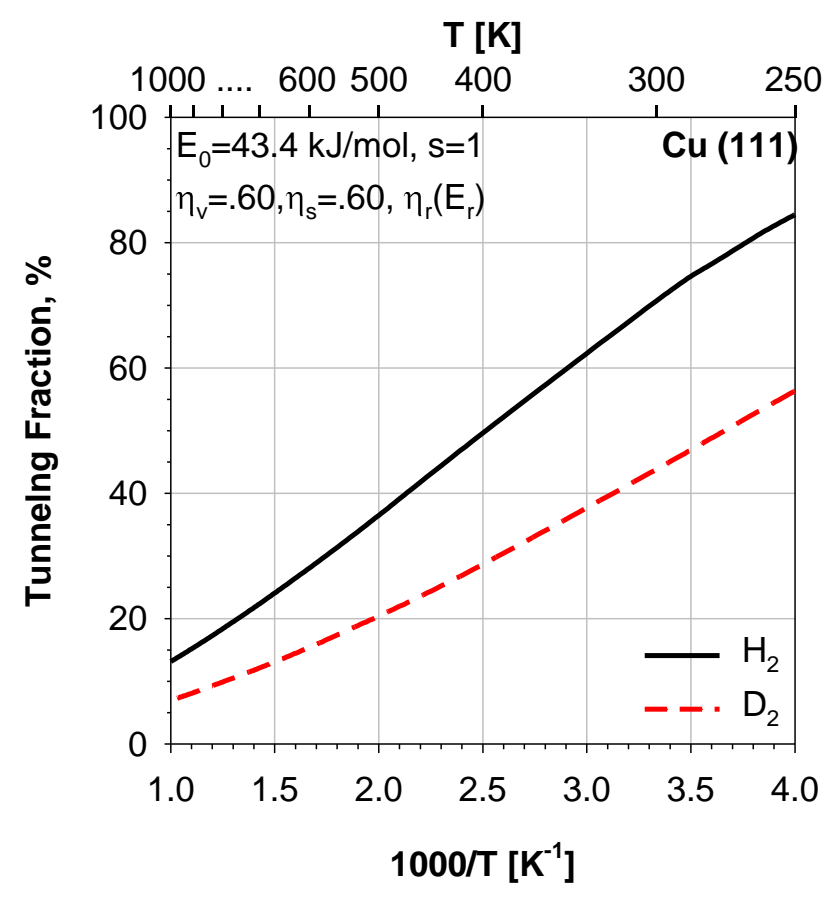

Figure 2.14: d-PMMT calculation of the fraction of the thermal dissociative sticking coefficient that derives from trajectories that tunnel through the energetic barrier to chemisorption. 

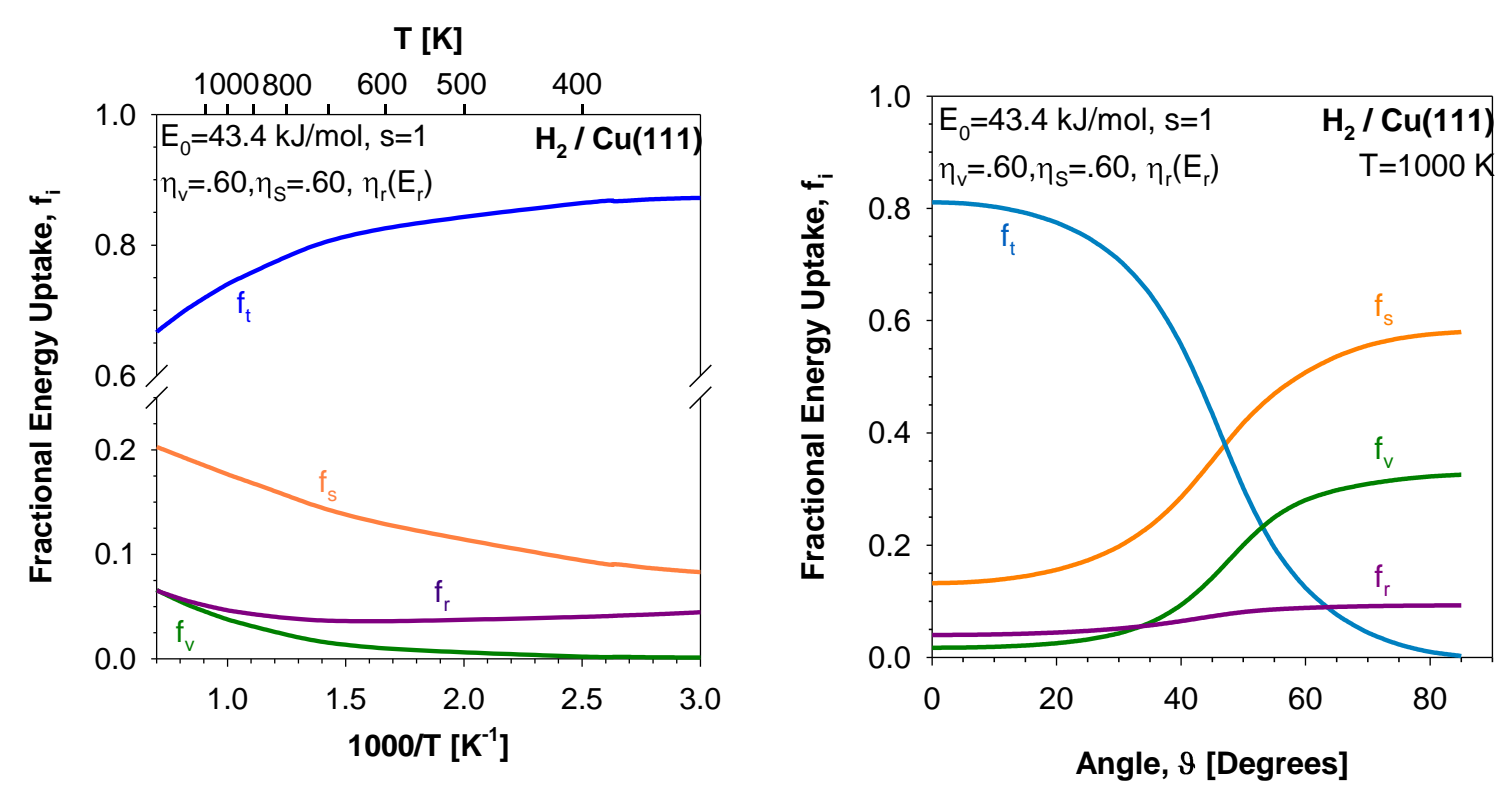

Figure 2.15: d-PMMT predictions of the fractional energy uptakes from the different degrees of freedom (e.g., $f_{i}=\left\langle\eta_{i} E_{i}\right\rangle_{R} /\left\langle E^{*}\right\rangle_{R}$ ) for thermal dissociative sticking (a) over the temperature range of interest to catalysis, and (b) as a function of angle of incidence at $1000 \mathrm{~K}$. 


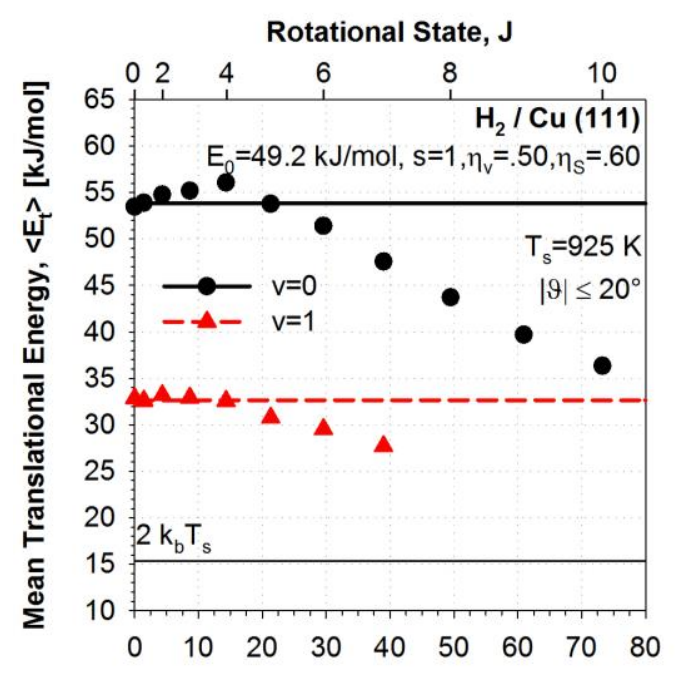

(a)

Rotational Energy, $E_{r}[\mathrm{~kJ} / \mathrm{mol}]$

Rotational State, J

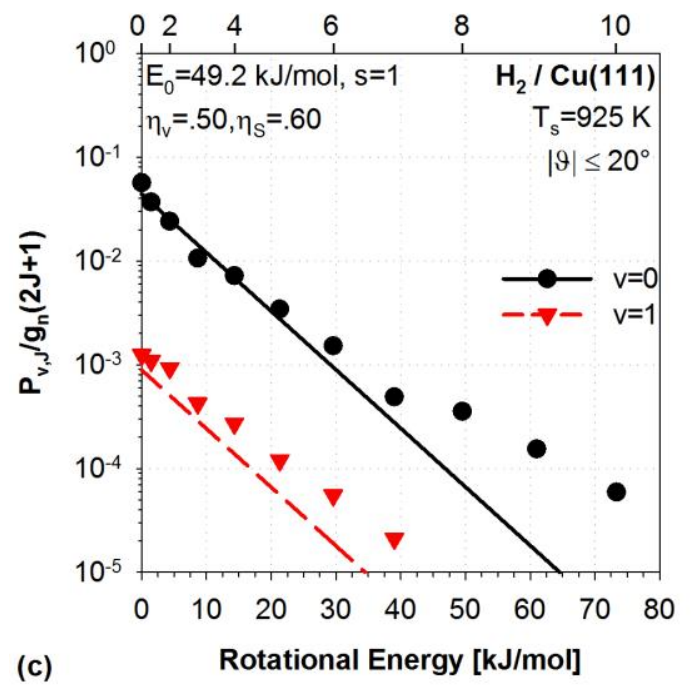

Rotational State, J

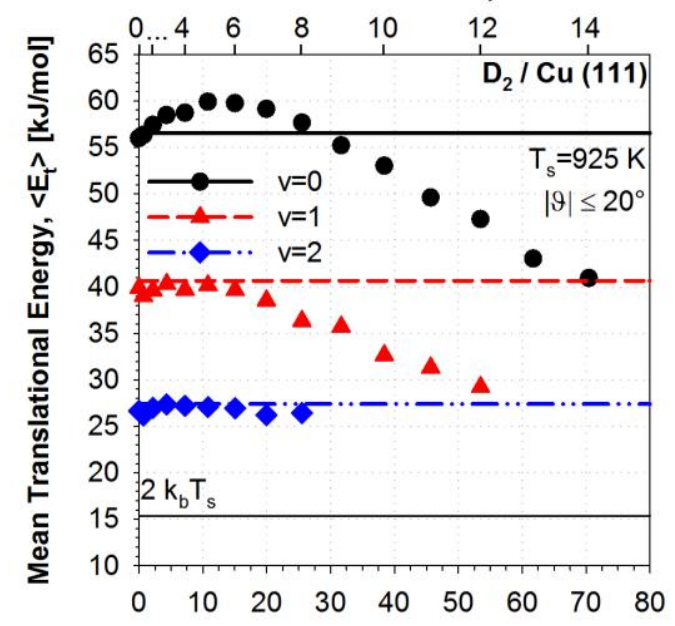

(b)

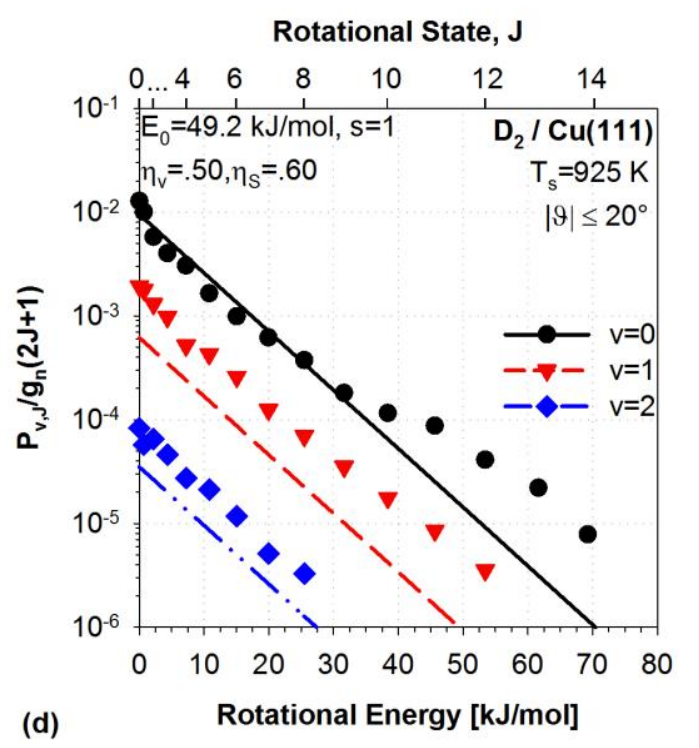

(d)

Rotational Energy [kJ/mol]

Figure 2.16: Measured rovibrational state resolved mean translational energies (points) ${ }^{6,7}$ for recombinatively desorbing (a) $\mathrm{H}_{2}$ and (b) $\mathrm{D}_{2}$ are compared to d-PMMT simulations (lines). A reference line demarking the $\left\langle E_{t}\right\rangle=2 k_{B} T_{s}$ expectation for a system exhibiting a dissociative sticking coefficient independent of $E_{t}$ is also provided. Boltzmann plots compare the measured vibrationally-resolved rotational energy distributions (points) ${ }^{6,7}$ for associative desorption of (c) $\mathrm{H}_{2}$ and (d) $\mathrm{D}_{2}$ to d-PMMT simulations (lines). 

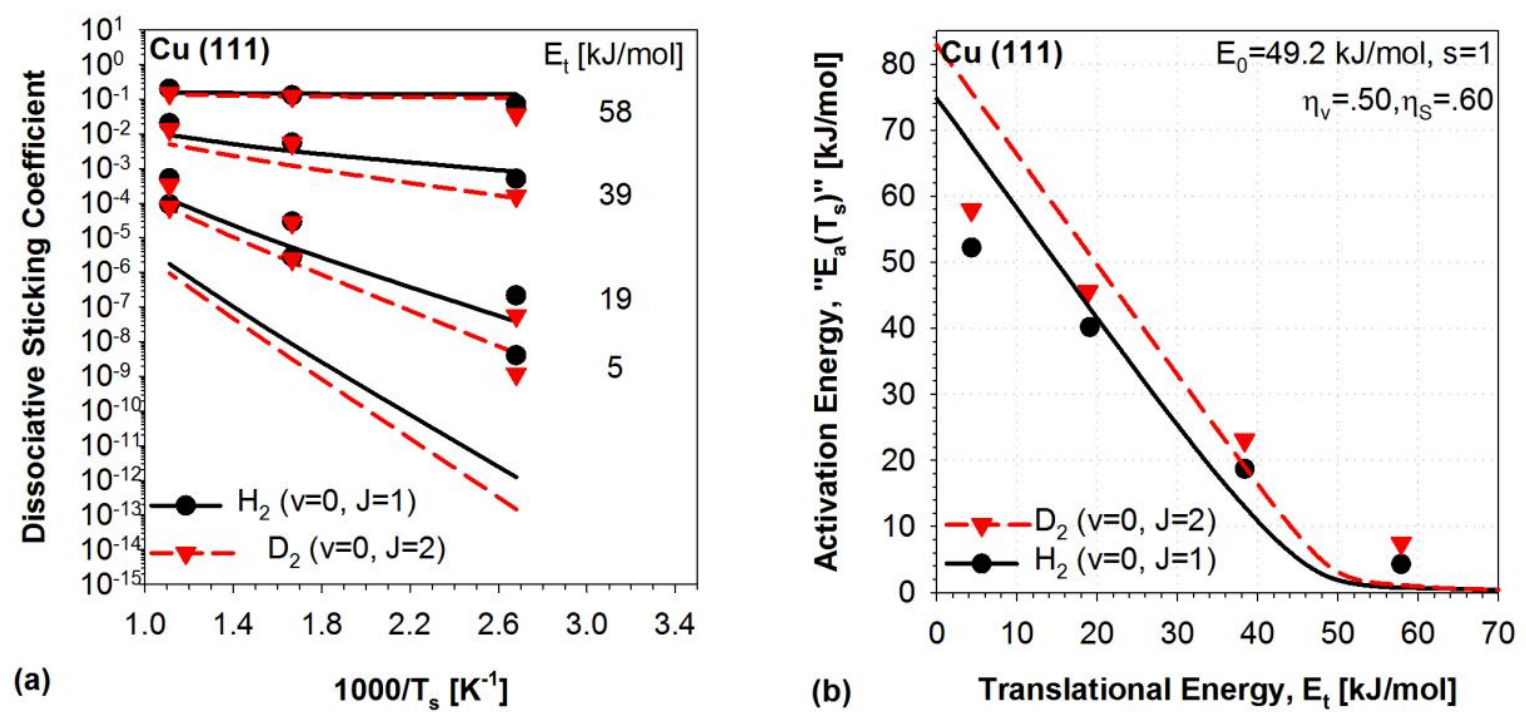

Figure 2.17: (a) Surface temperature dependent, rovibrational state resolved, relative dissociative sticking coefficients (points) ${ }^{16}$ derived from the measured associative desorption energy distributions of Fig. 2.9 are compared to d-PMMT simulations of absolute dissociative sticking coefficients (lines). (b) Effective activation energies derived from Arrhenius plots of the dissociative sticking coefficients shown in (a) are plotted for experiments (solid points) and dPMMT simulations (lines). 


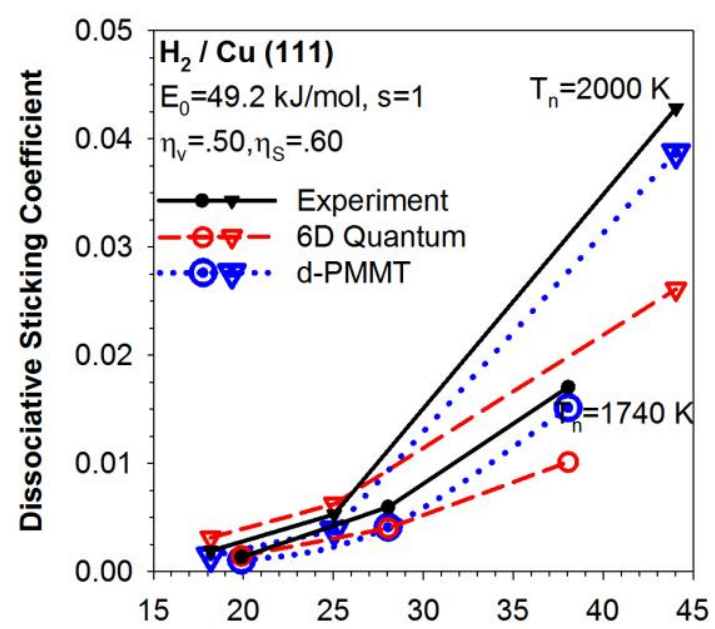

(a) Normal Translational Energy, $E_{n}[\mathrm{~kJ} / \mathrm{mol}]$

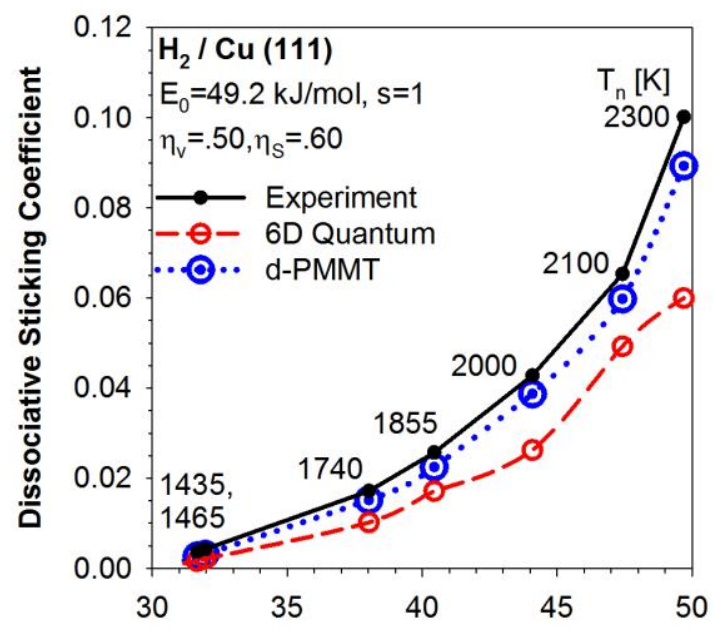

(b) Normal Translational Energy, $E_{n}[\mathrm{~kJ} / \mathrm{mol}]$

Figure 2.18: Non-equilibrium dissociative sticking coefficients for supersonic molecular beams of hydrogen impinging on $\mathrm{Cu}(111)$ (filled points) ${ }^{6}$ are compared to $6 \mathrm{D}$ quantum dynamics ${ }^{31,32}$ and d-PMMT simulations (open points). 

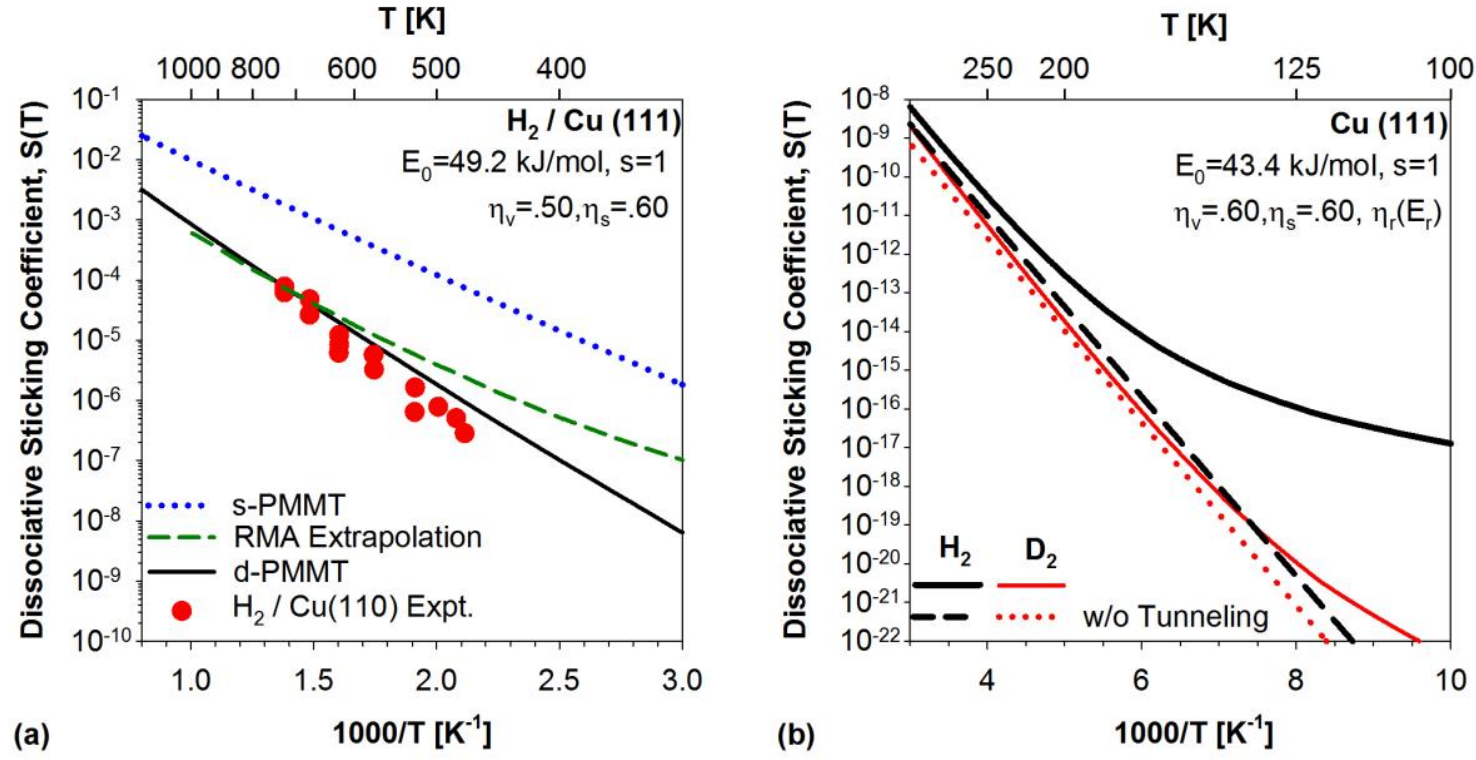

Figure 2.19: (a) Thermal dissociative sticking coefficient for $\mathrm{H}_{2} / \mathrm{Cu}(111)$ calculated by several models and experimental values ${ }^{58}$ for $\mathrm{H}_{2} / \mathrm{Cu}(100)$. (b) d-PMMT thermal dissociative sticking coefficients for $\mathrm{H}_{2}$ and $\mathrm{D}_{2}$ on $\mathrm{Cu}(111)$ are compared at lower temperatures where the effects of tunneling through the reaction barrier can be discerned (solid lines). Dashed lines give equivalent d-PMMT calculations without tunneling. 
References:

1. Bent, S. F.; Michelsen, H. A.; Zare, R. N., Hydrogen Recombinative Desorption Dynamics. In Laser Spectroscopy and Photochemistry on Metal Surfaces, Dai, H.-L.; Ho, W., Eds. World Scientific: Singapore, 1995.

2. Kroes, G. J., Six-dimensional quantum dynamics of dissociative chemisorption of H-2 on metal surfaces. Progress in Surface Science 1999, 60, (1-4), 1-85.

3. Michelsen, H. A.; Rettner, C. T.; Auerbach, D. J., The Adsorption of Hydrogen at Copper Surfaces: a Model System for the Study of Activated Adsorption. In Surface Reactions, Madix, R. J., Ed. Springer-Verlag: Berlin, 1994; Vol. 34, pp 185-237.

4. Berger, H. F.; Leisch, M.; Winkler, A.; Rendulic, K. D., A search for vibrational contributions to the activated adsorption of $\mathrm{H}_{2}$ on copper. Chemical Physics Letters 1990, 175, 425-428.

5. Anger, G.; Winkler, A.; Rendulic, K. D., Adsorption and Desorption-Kinetics in the Systems $\mathrm{H}-2 / \mathrm{Cu}(111), \mathrm{H}-2 / \mathrm{Cu}(110)$ and $\mathrm{H}-2 / \mathrm{Cu}(100)$. Surface Science 1989, 220, (1), 1-17.

6. Rettner, C. T.; Michelsen, H. A.; Auerbach, D. J., Quantum-State-Specific Dynamics of the Dissociative Adsorption and Associative Desorption of $\mathrm{H}_{2}$ at a $\mathrm{Cu}(111)$ Surface. J. Chem. Phys. 1995, 102, (11), 4625-4641.

7. Michelsen, H. A.; Rettner, C. T.; Auerbach, D. J.; Zare, R. N., Effect of Rotation on the Translational and Vibrational-Energy Dependence of the Dissociative Adsorption of $\mathrm{D}_{2}$ on $\mathrm{Cu}(111)$. J. Chem. Phys. 1993, 98, (10), 8294-8307.

8. Rettner, C. T.; Michelsen, H. A.; Auerbach, D. J.; Mullins, C. B., Dynamics of Recombinative Desorption - Angular-Distributions of $\mathrm{H}_{2}, \mathrm{HD}$ and $\mathrm{D}_{2}$ Desorbing from $\mathrm{Cu}(111) . J$. Chem. Phys. 1991, 94, (11), 7499-7501.

9. Auerbach, D. J.; Rettner, C. T.; Michelsen, H. A., Interaction Dynamics of Hydrogen at a $\mathrm{Cu}(111)$ Surface. Surf. Sci. 1993, 283, (1-3), 1-8.

10. Rettner, C. T.; Auerbach, D. J.; Michelsen, H. A., Role of Vibrational and Translational Energy in the Activated Dissociative Adsorption of D2 on Cu(111). Phys. Rev. Lett. 1992, 68, (8), 1164-1167.

11. Michelsen, H. A.; Rettner, C. T.; Auerbach, D. J., State-Specific Dynamics of D(2) Desorption from $\mathrm{Cu}(111)$ - the Role of Molecular Rotational Motion in Activated AdsorptionDesorption Dynamics. Phys. Rev. Lett. 1992, 69, (18), 2678-2681.

12. Rettner, C. T.; Michelsen, H. A.; Auerbach, D. J., Determination of Quantum-StateSpecific Gas-Surface Energy-Transfer and Adsorption Probabilities as a Function of KineticEnergy. Chem. Phys. 1993, 175, (1), 157-169.

13. Michelsen, H. A.; Auerbach, D. J., A Critical-Examination of Data on the Dissociative Adsorption and Associative Desorption of Hydrogen at Copper Surfaces. J. Chem. Phys. 1991, 94, (11), 7502-7520.

14. Hou, H.; Gulding, S. J.; Rettner, C. T.; Wodtke, A. M.; Auerbach, D. J., The stereodynamics of a gas-surface reaction. Science 1997, 277, (5322), 80-82.

15. Gulding, S. J.; Wodtke, A. M.; Hou, H.; Rettner, C. T.; Michelsen, H. A.; Auerbach, D. J., Alignment of $\mathrm{D}_{2}(\mathrm{v}, \mathrm{J})$ desorbed from $\mathrm{Cu}(111)$ : Low sensitivity of activated dissociative chemisorption to approach geometry. J. Chem. Phys. 1996, 105, (21), 9702-9705.

16. Murphy, M. J.; Hodgson, A., Adsorption and desorption dynamics of $\mathrm{H}_{2}$ and $\mathrm{D}_{2}$ on $\mathrm{Cu}(111)$ : The role of surface temperature and evidence for corrugation of the dissociation barrier. J. Chem. Phys. 1998, 108, (10), 4199-4211.

17. Comsa, G.; David, R., The Purely Fast Distribution of H-2 and D2 Molecules Desorbing from $\mathrm{Cu}(100)$ and $\mathrm{Cu}(111)$ Surfaces. Surface Science 1982, 117, (1-3), 77-84.

18. Gross, A.; Hammer, B.; Scheffler, M.; Brenig, W., High-dimensional quantum dynamics of adsorption and desorption of $\mathrm{H}_{2}$ at $\mathrm{Cu}(111)$. Physical Review Letters 1994, 73, 3121-3123. 
19. Sakong, S.; Gross, A., Dissociative adsorption of hydrogen on strained $\mathrm{Cu}$ surfaces. Surface Science 2003, 525, (1-3), 107-118.

20. Hammer, B.; Scheffler, M.; Jacobsen, K. W.; Norskov, J. K., Multidimensional Potential Energy Surface for $\mathrm{H}_{2}$ Dissociation over $\mathrm{Cu}(111)$. Physical Review Letters 1994, 73, 1400.

21. Kratzer, P.; Hammer, B.; Norskov, J. K., Geometric and electronic factors determining the differences in reactivity of $\mathrm{H}_{2}$ on $\mathrm{Cu}(100)$ and $\mathrm{Cu}(111)$. Surface Science 1996, 359, 45-53.

22. Nave, S.; Lemoine, D.; Somers, M. F.; Kingma, S. M.; Kroes, G. J., Six-dimensional quantum dynamics of $(\mathrm{v}=0, \mathrm{j}=0) \mathrm{D}-2$ and of $(\mathrm{v}=1, \mathrm{j}=0) \mathrm{H}-2$ scattering from $\mathrm{Cu}(111)$. J. Chem. Phys. 2005, 122, (21), -.

23. Somers, M. F.; Kingma, S. M.; Pijper, E.; Kroes, G. J.; Lemoine, D., Six-dimensional quantum dynamics of scattering of $(\mathrm{v}=0, \mathrm{j}=0) \mathrm{H}-2$ and $\mathrm{D}-2$ from $\mathrm{Cu}(111)$ : test of two LEPS potential energy surfaces. Chemical Physics Letters 2002, 360, (3-4), 390-399.

24. Wang, Z. S.; Darling, G. R.; Holloway, S., The surface temperature dependence of the inelastic scattering and dissociation of hydrogen molecules from metal surfaces. J. Chem. Phys. 2004, 120, (6), 2923-2933.

25. Wang, Z. S.; Darling, G. R.; Jackson, B.; Holloway, S., Test of approximations to surface motion in gas-surface dynamics: Linear versus quadratic coupling for T-s=0 K. J. Phys. Chem. B 2002, 106, (33), 8422-8428.

26. Dai, J. Q.; Light, J. C., The steric effect in a full dimensional quantum dynamics simulation for the dissociative adsorption of $\mathrm{H}-2$ on $\mathrm{Cu}(111)$. J. Chem. Phys. 1998, 108, (18), 7816-7820.

27. Dai, J. Q.; Light, J. C., Six dimensional quantum dynamics study for dissociative adsorption of H-2 on $\mathrm{Cu}(111)$ surface. J. Chem. Phys. 1997, 107, (5), 1676-1679.

28. Berger, H. F.; Leisch, M.; Winkler, A.; Rendulic, K. D., A Search for Vibrational Contributions to the Activated Adsorption of H-2 on Copper. Chemical Physics Letters 1990, $175,(5), 425-428$.

29. Kinnersley, A. D.; Darling, G. R.; Holloway, S., A comparison of quantum and classical dynamics of H-2 scattering from Cu(111). Surf. Sci. 1997, 377, (1-3), 563-566.

30. Darling, G. R.; Holloway, S., Rotational Motion and the Dissociation of H-2 on $\mathrm{Cu}(111)$. J. Chem. Phys. 1994, 101, (4), 3268-3281.

31. Diaz, C.; Pijper, E.; Olsen, R. A.; Busnengo, H. F.; Auerbach, D. J.; Kroes, G. J., Chemically Accurate Simulation of a Prototypical Surface Reaction: H(2) Dissociation on $\mathrm{Cu}(111)$. Science 2009, 326, (5954), 832-834.

32. Diaz, C.; Olsen, R. A.; Auerbach, D. J.; Kroes, G. J., Six-dimensional dynamics study of reactive and non reactive scattering of $\mathrm{H}-2$ from $\mathrm{Cu}(111)$ using a chemically accurate potential energy surface. Phys. Chem. Chem. Phys. 2010, 12, (24), 6499-6519.

33. Diaz, C.; Olsen, R. A.; Busnengo, H. F.; Kroes, G. J., Dynamics on Six-Dimensional Potential Energy Surfaces for $\mathrm{H}-2 / \mathrm{Cu}(111)$ : Corrugation Reducing Procedure versus Modified Shepard Interpolation Method and PW91 versus RPBE. J. Phys. Chem. C 2010, 114, (25), 1119211201.

34. Hand, M.; Harris, J., Recoil effects in surface dissociation. J. Chem. Phys. 1990, 92, (12), 7610-7617.

35. Dohle, M.; Saalfrank, P., Surface oscillator models for dissociative sticking of molecular hydrogen at non-rigid surfaces. Surf. Sci. 1997, 373, (1), 95-108.

36. Wang, Z. S.; Darling, G. R.; Holloway, S., Surface temperature dependence of the inelastic scattering of hydrogen molecules from metal surfaces. Phys. Rev. Lett. 2001, 87, (22), 226102.

37. Bonfanti, M.; Diaz, C.; Somers, M. F.; Kroes, G. J., Hydrogen dissociation on $\mathrm{Cu}(111)$ : the influence of lattice motion. Part I. Phys. Chem. Chem. Phys. 2011, 13, (10), 4552-4561. 
38. Donald, S. B.; Harrison, I., Dynamically biased RRKM model of activated gas-surface reactivity: vibrational efficacy and rotation as a spectator in the dissociative chemisorption of $\mathrm{CH}_{4}$ on Pt(111). Phys. Chem. Chem. Phys. 2012, 14, (5), 1784-1796.

39. Navin, J. K.; Donald, S. B.; Tinney, D. G.; Cushing, G. W.; Harrison, I., Communication: Angle-resolved thermal dissociative sticking of $\mathrm{CH}_{4}$ on $\mathrm{Pt}(111)$ : Further indication that rotation is a spectator to the gas-surface reaction dynamics. J. Chem. Phys. 2012, 136, 061101.

40. Donald, S. B.; Harrison, I., Methane Dissociative Chemisorption Dynamics and Detailed Balance on Pt(111) and Ru(0001). J. Chem. Phys. 2012, to be submitted.

41. Forst, W., Unimolecular Reactions: A Concise Introduction. 1st ed.; Cambridge University Press: Cambridge UK, 2003.

42. Juurlink, L. B. F.; Killelea, D. R.; Utz, A. L., State-resolved probes of methane dissociation dynamics. Prog. Surf. Sci. 2009, 84, (3-4), 69-134.

43. Juurlink, L. B. F.; Smith, R. R.; Utz, A. L., The role of rotational excitation in the activated dissociative chemisorption of vibrationally excited methane on $\mathrm{Ni}(100)$. Faraday Discuss. 2000, 117, 147-160.

44. Herzberg, G., Molecular Spectra and Molecular Structure I. Spectra of Diatomic Molecules. $2^{\text {nd }}$ ed.; van Nostrand Reinhold Company: New York, 1950.

45. Stein, S. E.; Rabinovitch, B. S., Accurate evaluation of internal energy level sums and densities including anharmonic oscillators and hindered rotors. J. Chem. Phys. 1973, 58, (6), 2438 .

46. Miller, W. H., Tunneling corrections to unimolecular rate constants, with application to formaldehyde. J. Am. Chem. Soc. 1979, 101, (23), 6810-6814.

47. Baer, T.; Hase, W. L., Unimolecular Reaction Dynamics. Oxford University Press: New York, NY, 1996.

48. Johnston, H. S.; Heicklen, J., Tunnelling corrections for unsymmetrical Eckart potential energy barriers. J. Phys. Chem. 1962, 66, (3), 532-533.

49. Booze, J. A.; Weitzel, K. M.; Baer, T., The rates of $\mathrm{HCl}$ loss from energy-selected ethylchloride ions - a case of tunneling through an H-atom transfer barrier. J. Chem. Phys. 1991, 94, (5), 3649-3656.

50. Abbott, H. L.; Harrison, I., Seven-dimensional microcanonical treatment of hydrogen dissociation dynamics on $\mathrm{Cu}(111)$ : Clarifying the essential role of surface phonons. J. Chem. Phys. 2006, 125, (2), 024704.

51. Abbott, H. L.; Harrison, I., Microcanonical transition state theory for activated gassurface reaction dynamics: Application to $\mathrm{H}_{2} / \mathrm{Cu}(111)$ with rotation as a spectator. J. Phys. Chem. A 2007, 111, (39), 9871-9883.

52. Cardillo, M. J.; Balooch, M.; Stickney, R. E., Detailed Balancing and Quasi-equilibrium in the Adsorption of Hydrogen on Copper. Surf. Sci. 1975, 50, 263-278.

53. Gross, A.; Hammer, B.; Scheffler, M.; Brenig, W., High-Dimensional Quantum Dynamics Of Adsorption And Desorption $\mathrm{Of}_{2} \mathrm{At} \mathrm{Cu}(111)$. Phys. Rev. Lett. 1994, 73, (23), 3121-3124.

54. Comsa, G.; David, R., The Purely Fast Distribution of $\mathrm{H}_{2}$ and $\mathrm{D}_{2}$ Molecules Desorbing from $\mathrm{Cu}(100)$ and $\mathrm{Cu}(111)$ Surfaces. Surf. Sci. 1982, 117, (1-3), 77-84.

55. Van Willigen, W., Angular distribution of hydrogen molecules desorbed from metal surfaces. Phys. Lett. A 1968, 28, (2), 80-81.

56. Hodgson, A., State resolved desorption measurements as a probe of surface reactions. Prog. Surf. Sci. 2000, 63, (1-2), 1-61.

57. Bukoski, A.; Blumling, D.; Harrison, I., Microcanonical unimolecular rate theory at surfaces. I. Dissociative chemisorption of methane on Pt(111). J. Chem. Phys. 2003, 118, (2), 843-871. 
58. Campbell, J. M.; Campbell, C. T., The dissociative adsorption of $\mathrm{H}_{2}$ and $\mathrm{D}_{2}$ on $\mathrm{Cu}(110)$ activation barriers and dynamics. Surf. Sci. 1991, 259, (1-2), 1-17.

59. Rettner, C. T.; Michelsen, H. A.; Auerbach, D. J., From Quantum-State-Specific Dynamics to Reaction-Rates - the Dominant Role of Translational Energy in Promoting the Dissociation of $\mathrm{D}_{2}$ on $\mathrm{Cu}(111)$ under Equilibrium Conditions. Faraday Discuss. 1993, 96, 17-31.

60. Abbott, H. L.; Harrison, I., Activated dissociation of $\mathrm{CO}_{2}$ on $\mathrm{Rh}(111)$ and $\mathrm{CO}$ oxidation dynamics. J. Phys. Chem. C 2007, 111, (35), 13137-13148.

61. Balooch, M.; Cardillo, M. J.; Miller, D. R.; Stickney, R. E., Molecular beam study of the apparent activation barrier associated with adsorption and desorption of hydrogen on copper. Surf. Sci. 1974, 46, (2), 358-392.

62. Rettner, C. T.; Delouise, L. A.; Auerbach, D. J., Effect Of Incidence Kinetic-Energy And Surface Coverage On The Dissociative Chemisorption Of Oxygen On W(110). J. Chem. Phys. 1986, 85, (2), 1131-1149.

63. Luntz, A. C., A simple model for associative desorption and dissociative chemisorption. J. Chem. Phys. 2000, 113, (16), 6901-6905.

64. Levine, R. D.; Bernstein, R. B., Energy disposal and energy consumption in elementary chemical-reactions - information theoretic approach. Acc. Chem. Res. 1974, 7, (12), 393-400.

65. Steinfeld, J. I.; Francisco, J. S.; Hase, W. L., Chemical kinetics and dynamics. 2nd ed.; Prentice-Hall: Upper Saddle River, N.J., 1998.

66. Halstead, D.; Holloway, S., The Influence of Potential-Energy Surface Topologies on the Dissociation of $\mathrm{H}_{2}$. J. Chem. Phys. 1990, 93, (4), 2859-2870. 
3.

\title{
RRKM Simulation of Methane Dissociation on $\operatorname{Pt}(111)$ :
}

\section{Addressing Vibrational Efficacy and Spectator Rotation}

\begin{abstract}
The reactivity of $\mathrm{CH}_{4}$ impinging on a $\mathrm{Pt}(111)$ surface was examined using a precursormediated microcanonical trapping model of dissociative chemisorption wherein the effects of rotational and vibrational energy could be explored. Dissociative sticking coefficients for a diverse range of non-equilibrium effusive beam, supersonic beam, and eigenstate-resolved experiments were simulated and an average relative discrepancy between theory and experiment of better than $50 \%$ was achieved by treating molecular rotations and translation parallel to the surface as spectator degrees of freedom, and introducing a dynamically-biased vibrational efficacy. The model parameters are $\left\{E_{0}=58.9 \mathrm{~kJ} / \mathrm{mol}, s=2, \eta_{v}=0.40\right\}$ where $E_{0}$ is the apparent threshold energy for reaction, $s$ is the number of surface oscillators participating in energy exchange within each gas-surface collision complex formed, and $\eta_{v}$ is the mean vibrational efficacy for reaction relative to normal translational energy which figures in the assembly of the active exchangeable energy which is available to surmount the activation barrier to dissociative chemisorption. GGA-DFT electronic structure calculations provided vibrational frequencies for the transition state for dissociative chemisorption. The asymmetry of the rotational state populations in supersonic and effusive molecular beam experiments allowed kinetic analysis to establish that taking rotation as a spectator degree of freedom is a good approximation. Surface phonons, rather than the incident molecules, are calculated to play the dominant role in supplying the energy required to overcome the activation barrier for dissociative chemisorption under the thermal equilibrium conditions relevant to high pressure catalysis. Over the temperature range $300 \mathrm{~K} \leq T \leq 1000 \mathrm{~K}$, the thermal dissociative sticking coefficient is predicted to be well described by $S(T)=S_{0} \exp \left(-E_{a} / R T\right)$ where $S_{0}=0.62$ and $E_{a}=64.7 \mathrm{~kJ} / \mathrm{mol}$.
\end{abstract}




\subsection{Introduction}

Dissociative chemisorption can be the rate-limiting step in some of society's most important industrial activities relating to energy, commodity chemicals, and advanced materials. ${ }^{1-}$

${ }^{5}$ In this chapter, a dynamically-biased transition state theory model of activated dissociative chemisorption is described that provides one approach to bridge the divide between electronic structure theory calculations and accurate prediction of non-equilibrium and thermal dissociative sticking coefficients at surfaces. The fundamental questions of whether molecular rotation can be approximated as a spectator degree of freedom and which gas-surface degrees of freedom are most important in providing the energy required to overcome the activation barrier for dissociative chemisorption of methane on Pt(111) are addressed.

Activated dissociative chemisorption is a fundamental reaction that can be the ratedetermining step in catalytic ${ }^{6}$ and chemical vapor deposition ${ }^{7}$ processes of industrial importance. Moreover, this kind of gas-surface reaction provides unusually diverse experimental opportunities to test theoretical models of reactivity at surfaces because the gas-phase reactants can be prepared in specific initial states prior to their encounter with the surface. In the process of activated dissociative chemisorption a molecule approaching a surface typically encounters a precursor molecular adsorption well based on van der Waals and/or chemical forces exerted between the intact molecule and the surface before it is able to gain access to the transition state region for activated molecular dissociation. ${ }^{8}$ A precursor-mediated microcanonical trapping model $^{9-11}$ (PMMT) positing that energy becomes microcanonically randomized in the local gas-surface collision complexes formed in the configurational vicinity of the precursor potential well (i.e., trapped between the transition states to dissociative chemisorption and molecular desorption) has proven useful in describing the reaction dynamics and kinetics for the activated dissociative chemisorption of $\mathrm{H}_{2} / \mathrm{Cu}(111),{ }^{12} \mathrm{CO}_{2} / \mathrm{Rh}(111),{ }^{13} \mathrm{SiH}_{4}$ on $\mathrm{Si}(100),{ }^{14}$ light alkanes on $\mathrm{Pt}(111),{ }^{15}, 16$ etc. The microcanonical unimolecular rate theory (MURT) approach provides statistical baseline 
predictions for gas-surface reactivity against which dynamical effects can be experimentally recognized. Certainly, some dynamical (non-statistical) behavior has been experimentally observed in the activated dissociative chemisorption of some small molecule/surface systems, for example, $\mathrm{H}_{2} / \mathrm{Cu}(111),{ }^{17}$ and methane ${ }^{18}$ on $\mathrm{Ni}(100),{ }^{19} \mathrm{Ni}(111),{ }^{20}$ and $\mathrm{Pt}(111) .{ }^{16,}{ }^{21}$ Here, we begin to address several open questions for the $\mathrm{CH}_{4} / \mathrm{Pt}(111)$ system. What is the information content of the dynamical findings and how do they impact the ability to predict the outcomes of other experiments? Can the experimental behavior be largely summarized by a statistical model subject to a few dynamical constraints? What dynamical constraints must be known in order to use the findings of high signal-to-noise nonequilibrium experiments to predict other experiments, and, in particular, the thermal equilibrium dissociative sticking coefficients relevant to high pressure catalysis? Given that direct dynamical simulations ${ }^{22}$ of dissociative chemisorption of polyatomic molecules on metal surfaces have thus far been unable to quantitatively reproduce or predict experimental results due to the high dimensionality of the reactions and the typically $\pm 30 \mathrm{~kJ} / \mathrm{mol}$ uncertainties $^{23}$ in potential energy surfaces calculated by electronic structure theory methods (vide infra), it is important to explore alternative theoretical means to predict and analyze experimental outcomes.

In favorable cases, the state distributions of the gaseous products of thermally-driven associative desorption (the time reversal of dissociative chemisorption) can provide information about dissociative sticking through the principle of detailed balance. ${ }^{24,} 25$ For hydrogen dissociative chemisorption on $\mathrm{Cu}(111)$, detailed balance applied to associative desorption has shown that molecular vibrational energy and rotational energy are not as efficacious in promoting dissociative chemisorption as either molecular translational energy directed along the surface normal, ${ }^{17,26}$ or surface thermal energy. ${ }^{27}$ Studies of methane dissociative chemisorption on metals have also shown that energy from both the incident molecules ${ }^{28,29}$ and the surface ${ }^{30}$ can promote reactivity. Laser-pumped, supersonic molecular beam experiments measuring state-resolved 
dissociative sticking coefficients have demonstrated vibrational mode-specific reactivity for methane on several metal surfaces. ${ }^{19-21}$ More difficult has been to determine the influence of rotational energy on the reactivity of polyatomic gas-surface reactions, in part because sufficiently sensitive state-resolved detection schemes to probe associatively desorbing polyatomic molecules have been lacking. ${ }^{25}$ For direct dissociative sticking experiments, laser pumping of supersonic molecular beams cannot easily prepare molecules in states with high, or broadly tunable, rotational quantum numbers, $J$, because rotational cooling in the supersonic expansion leaves most molecules with $J$ values near 0 and typical optical selection rules are only $\Delta J= \pm 1$ or $\pm 2 .{ }^{31}$ An alternative approach to assess the role of rotational energy in polyatomic gas-surface reactivity is to compare the reactivity of molecules prepared in effusive and supersonic molecular beams with comparable vibrational temperatures but very different rotational temperatures. ${ }^{32}$ Molecules prepared in an effusive beam have a flux-weighted Maxwell Boltzmann energy distribution whose gas temperature, $T_{g}=T_{t}=T_{v}=T_{r}$, is shared by all molecular degrees of freedom and is set by the beam's nozzle temperature. ${ }^{33}$ Methane molecules prepared in a supersonic beam with nozzle temperature $T_{N}$ suffer negligible vibrational cooling, $T_{v}=T_{N}$, but substantial rotational cooling, $T_{r}=0.1 T_{N}$, due to inter-molecular collisions during the supersonic expansion. ${ }^{30}$

In this chapter, a dynamical biasing constraint is incorporated into the statistical (s-) PMMT model of dissociative chemisorption in the spirit of information-theoretic/maximum entropy approaches ${ }^{34,35}$ more commonly used in gas-phase reaction dynamics in which dynamical constraints are imposed on otherwise statistical models. A dynamically-biased (d-) PMMT model of the $\mathrm{CH}_{4} / \mathrm{Pt}(111)$ reactivity, incorporating the experimentally derived biasing constraint that vibrational energy is less efficacious than normal translational energy in promoting reaction, as well as the assertion that molecular rotations and translation parallel to the surface are spectator 
degrees of freedom, is shown to quantitatively reproduce a diverse range of experimental results with an average relative discrepancy (ARD) of $43 \%$. For the first time, a single theoretical model is able to reproduce dissociative sticking coefficients for both supersonic and effusive molecular beam experiments - experiments for which the rotational distributions of the incident molecules differ dramatically because of collisionally-mediated rotational cooling in the expansion of the supersonic beam. PMMT modeling of these complimentary kinds of beam experiments with asymmetric rotational populations makes it possible to assert that, to a good approximation, rotation can be treated as a spectator to the reaction dynamics. This is an important conceptual finding that decreases calculated thermal dissociative sticking coefficients based on the results of non-equilibrium molecular beam experiments by roughly an order of magnitude. Over the temperature range $300 \mathrm{~K} \leq T \leq 1000 \mathrm{~K}$, the thermal dissociative sticking coefficient calculated by the d-PMMT model is well described by $S(T)=S_{0} \exp \left(-E_{a} / R T\right)$ where $S_{0}=0.62$ and $E_{a}=62.6 \mathrm{~kJ} / \mathrm{mol}$. The surface phonons, and not the incident molecules, are calculated to play the dominant role in supplying the energy required to overcome the activation barrier for $\mathrm{CH}_{4} / \mathrm{Pt}(111)$ dissociative chemisorption under the thermal equilibrium conditions relevant to high pressure catalysis. These energy supply roles can be reversed in non-equilibrium dissociative chemisorption experiments if sufficiently hyperthermal molecular beams are made incident on the surface. The d-PMMT model is shown to provide a useful kinetic and conceptual framework, grounded in transition state theory, to analyze and predict dissociative sticking coefficients for activated dissociative chemisorption over a diverse range of experimental conditions.

\subsection{Precursor Mediated Microcanonical Trapping Theory}

\subsection{A s-PMMT Model}


A schematic summary of the s-PMMT model ${ }^{9-11}$ for activated dissociative chemisorption is presented in Figure 3.1. Briefly, gas-surface collision complexes formed from an incoming molecule and a few local surface oscillators with a pooled energy sufficient to react are assumed to have their energy microcanonically randomized through state-mixing collisions with the reactive potential ${ }^{36}$ and/or intramolecular vibrational energy redistribution (IVR) ${ }^{9}$ such that the molecules become trapped in the precursor physisorption well located between the transition states for reaction (dissociative chemisorption) and desorption. These precursor complexes (PCs) go on to react or desorb with Rice-Ramsperger-Kassel-Marcus (RRKM) rate constants,

$$
k_{i}(E)=\frac{W_{i}^{\dagger}\left(E-E_{i}\right)}{h \rho(E)}
$$

where $E$ is the active exchangeable energy, $W_{i}^{\dagger}\left(E-E_{i}\right)$ is the sum of states for transition state $i=D, R$ with threshold energy for reaction $E_{i}$ (e.g., $E_{R}=E_{D}+E_{0}$ in Fig. 3.1), $\rho(E)$ is the PC density of states, and $h$ is Planck's constant. On the oftentimes convenient $E^{*}=E-E_{D}$ energy scale, whose zero occurs for the reactants at infinite separation, the apparent threshold energy for reaction is $E_{0}$. Lifetimes for molecular desorption at reactive energies $\left(E^{*} \geq E_{0}\right)$ are ultrafast for methane on $\operatorname{Pt}(111)$ which has a relatively shallow physisorption well, $E_{D}=16 \mathrm{~kJ} / \mathrm{mol},{ }^{37}$ and a high threshold energy for dissociative chemisorption, $E_{0} \sim 58 \mathrm{~kJ} / \mathrm{mol}$. In this instance, ignoring energy exchange between the PCs and the surrounding metal bulk is a good approximation ${ }^{9,11,15}$ and yields what has often been called the physisorbed complex-microcanonical unimolecular rate theory (PC-MURT) model of dissociative chemisorption which we will use here as our s-PMMT model.

Applying the steady state approximation to the simplified Fig. 3.1 kinetics scheme, ${ }^{9,36}$ 


$$
\mathrm{CH}_{4(g)} \underset{k_{D}\left(E^{*}\right)}{\stackrel{F_{0} f\left(E^{*}\right)}{\rightleftarrows}} \mathrm{CH}_{4(p)} \stackrel{k_{R}\left(E^{*}\right)}{\longrightarrow} \mathrm{CH}_{3(c)}+H_{(c)}
$$

yields the following expression for the experimental dissociative sticking coefficient,

$$
S=\int_{0}^{\infty} S\left(E^{*}\right) f\left(E^{*}\right) d E^{*}
$$

where $S\left(E^{*}\right)$ is the microcanonical sticking coefficient,

$$
S\left(E^{*}\right)=\frac{k_{R}\left(E^{*}\right)}{k_{R}\left(E^{*}\right)+k_{D}\left(E^{*}\right)}=\frac{W_{R}^{\dagger}\left(E^{*}-E_{0}\right)}{W_{R}^{\dagger}\left(E^{*}-E_{0}\right)+W_{D}^{\dagger}\left(E^{*}\right)}
$$

and $f\left(E^{*}\right)$ is the probability distribution,

$f\left(E^{*}\right)=\int_{0}^{E^{*}} f_{r}\left(E_{r}\right) \int_{0}^{E^{*}-E_{r}} f_{v}\left(E_{v}\right) \int_{0}^{E^{*}-E_{v}-E_{r}} f_{n}\left(E_{n}\right) \times f_{s}\left(E^{*}-E_{n}-E_{v}-E_{r}\right) d E_{n} d E_{v} d E_{r}$

for forming a PC with energy $E^{*}$ which is calculated by convolution over the molecular and surface energy distributions describing the particular experimental conditions of interest. The experimental sticking coefficient is the average of the microcanonical sticking coefficient over the experimental probability of forming a PC with energy $E^{*}$. Worth noting is that the microcanonical sticking coefficient of Eq.(3.4) is simply the ratio of the number of open channels to react to the total number of open channels to either react or desorb. This is a completely statistical result and is consistent with the "prior" expectation of information-theoretic/maximum entropy modeling approaches. ${ }^{34,35}$

Dissociative sticking coefficients for $\mathrm{CH}_{4} / \mathrm{Pt}(111)$ are experimentally ${ }^{30,38}$ found to scale with the normal component of the molecular translational energy, or normal translational energy, $E_{n}=E_{t} \cos ^{2} \vartheta$, where $\vartheta$ is the molecular angle of incidence to the surface measured away from 
the surface normal. In consequence, molecular translational energy parallel to the surface is taken to be a spectator degree of freedom. Energy associated with all other molecular degrees of freedom and the energy of $s$ surface oscillators is assumed to be active exchangeable energy within the PCs formed. Dissociative sticking calculations require specification of the transition states for desorption and reaction. The desorption transition state was taken to occur when the alkane is freely rotating and vibrating in the gas-phase, far from the $s$ surface oscillators of the united PC. The surface oscillators are assumed to vibrate at the mean phonon frequency of the metal $\left[v_{s}=\left(\frac{3}{4}\right) k_{B} T_{\text {Debye }} / h\right.$ yielding $122 \mathrm{~cm}^{-1}$ for $\left.\mathrm{Pt}\right]$ with only the component of the lattice vibrations normal to the surface contributing to the PC exchangeable energy (symmetric with the experimental finding that the dissociative sticking coefficient scales with only the normal component of the molecular translational energy). The desorption coordinate was taken to be the vibrational motion that ultimately becomes free molecular translation along the surface normal, and so that degree of freedom is missing from the desorption transition state (n.b., also missing are the spectator translational motions parallel to the surface). The transition state for dissociative chemisorption was defined by the vibrational frequencies calculated by Nave, Tiwari, and Jackson $^{39}$ using generalized gradient approximation - density functional theory (GGA-DFT) electronic structure theory but the apparent threshold energy for reaction was treated as a free variable. In this way, only 2 parameters are required for s-PMMT calculations, $\left\{E_{0}, s\right\}$, where $E_{0}$ is the apparent threshold energy for dissociative chemisorption (see Fig. 3.1) and $s$ is the number of surface oscillators. These parameters were fixed by minimizing the average relative discrepancy, ARD, between simulated theoretical and experimental dissociative sticking coefficients,

$$
A R D=\left\langle\frac{\left|S_{\text {theory }}-S_{\text {expt }}\right|}{\min \left(S_{\text {theory }}, S_{\text {exp } t}\right)}\right\rangle
$$


for a limited set of nonequilibrium supersonic molecular beam experiments performed by Luntz and Bethune $\mathrm{e}^{30}$ at two different nozzle temperatures. Once the model parameters were defined it was possible to simulate dissociative sticking coefficients for any experiment for which the initial energy distributions are known. For supersonic molecular beam experiments involving a thermal nozzle at temperature, $T_{N}$, it was assumed that the vibrational temperature of the beam molecules was fixed by the nozzle temperature, $T_{v}=T_{N}$, and that rotational cooling in the supersonic expansion left the beam molecules with a rotational temperature of $T_{r}=0.1 T_{N}$.

\subsection{B. d-PMMT Model}

Early $\mathrm{CH}_{4} / \mathrm{Pt}(111)$ supersonic beam experiments ${ }^{30}$ with varying $T_{N}$ provided some indication that molecular vibrational energy, $E_{v}$, was not as efficacious in promoting reaction as normal translational energy. Recent $\mathrm{CH}_{4} / \mathrm{Pt}(111)$ laser-pumped supersonic molecular beam experiments by Beck and co-workers ${ }^{21}$ measured eigenstate-resolved dissociative sticking coefficients and determined a vibrational efficacy for promoting reaction relative to $E_{n}$ of $\eta_{v}=$ 0.38 when $2 v_{3}$ quanta of asymmetric $\mathrm{C}-\mathrm{H}$ stretch vibrational energy, amounting to $\Delta E_{v}=72$ $\mathrm{kJ} / \mathrm{mol}$, was introduced to the incident molecular beam. Eigenstate-resolved experiments for methane dissociative chemisorption on several metal surfaces have determined a range of relative vibrational efficacies, $0.4 \leq \eta_{v} \leq 1.4$, that vary with the vibrational mode and metal surface. ${ }^{18}$ The value of $\eta_{v}$ appropriate to purely statistical transition state theories of chemical reactivity is $\eta_{v}=1$ and here we work to implement a d-PMMT that is constrained to uphold a single value of $\eta_{v}$ for a given reactive system (i.e., $\eta_{v}$ will be an average value). 
In the gas-phase, most polyatomic molecule reactions are believed to occur according to statistical transition state theory. ${ }^{40}$ Our understanding of state-selective polyatomic reactions ${ }^{41}$ is much less developed than for atom + diatomic molecule reactions for which the Polanyi rules ${ }^{42}$ apply. Polanyi found that translational energy was more helpful in surmounting an early barrier on the reactive potential energy surface (PES) and vibrational energy was more helpful in surmounting a late barrier. For classical trajectories on an elbow plot, especially for a late barrier, Polanyi also found that the phase of the vibrational excitation was an important additional variable in determining whether or not a trajectory would result in a successful atom + diatomic molecule reaction or rather in inelastic scattering. Presumably for some multidimensional polyatomic molecule + surface reaction dynamics, similar early/late barrier energy preferences hold true. The key issue is the efficiency of conversion of the different forms of reactant energy into energy that can be used to surmount the potential energy barrier to reaction.

Given that vibrational energy is not a spectator degree of freedom to the gas-surface reaction dynamics and the open channels of the microcanonical sticking coefficient in Eq. (3.4) are fixed by the modal structure of the transition states, it is only in the assembly of the active exchangeable energy that we can easily impose a dynamical constraint on the initial s-PMMT model. For the sake of notational simplicity in the equations below, we assume that rotation is a spectator to the chemisorption dynamics in addition to translational energy parallel to the surface. Let us first define the active exchangeable energy as,

$$
E^{*}=\varepsilon_{n} E_{n}+\varepsilon_{v} E_{v}+\varepsilon_{s} E_{s}
$$

where the $\varepsilon_{i} \leq 1$ are efficiencies for conversion of energy $E_{i}$ to active exchangeable energy $E^{*}$ capable of surmounting the activation barrier to reaction. According to Eq. (3), the incremental 
change in the experimental dissociative sticking coefficient due to the sticking at energy $E^{*}$ in increment $d E^{*}$ is,

$$
\begin{aligned}
d S & =S\left(E^{*}\right) f\left(E^{*}\right) d E^{*} \\
& =\left.S\left(E^{*}\right) f\left(E^{*}\right) \sum_{i} \frac{\partial E^{*}}{\partial E_{i}}\right|_{E_{j \neq i}} d E_{i}
\end{aligned}
$$

Consequently, the vibrational efficacy of reaction relative to normal translational energy is,

$$
\begin{aligned}
\eta_{v} & \left.\left.\equiv \frac{\partial S}{\partial E_{v}}\right|_{E_{j \neq v}} \frac{\partial S}{\partial E_{n}}\right|_{E_{j \neq n}} ^{-1}=\left.\left.\frac{\partial E^{*}}{\partial E_{v}}\right|_{E_{j \neq v}} \frac{\partial E^{*}}{\partial E_{n}}\right|_{E_{j \neq n}} ^{-1} \\
& =\frac{\varepsilon_{v}}{\varepsilon_{n}}
\end{aligned}
$$

If the Polanyi rules are applicable to relatively direct ${ }^{43}$ gas-surface reactions, we might expect $\varepsilon_{n} \sim 1$ and $\varepsilon_{v}<1$ for an early barrier such that $\eta_{v}<1$, whereas complementary efficiency values should apply for a late barrier leading to $\eta_{v}>1 .^{44}$ Although the gas-surface collisions are generally presumed to be efficiently state-mixing at energies sufficient to access the transition state region of the reactive potential energy surface, it could be that some gas-phase molecular vibrational modes project out, during collision with the surface, entirely or partially on to spectator degrees of freedom for dissociative chemisorption (e.g., parallel translations, rotations, or, perhaps, some specific vibrational modes ${ }^{45}$ ). In the absence of experimental information to the contrary the statistical assumption is to assume that all the $\varepsilon_{i}=1$. For the $\mathrm{CH}_{4} / \mathrm{Pt}(111)$ system there is experimental evidence that $\eta_{v}<1$ and assuming $\varepsilon_{n}=\varepsilon_{s}=1$ we can rewrite Eq.(3.7) with a single parameter,

$$
E^{*}=E_{n}+\eta_{v} E_{v}+E_{s}
$$


The probability distribution, $f\left(E^{*}\right)$, for forming a PC with this exchangeable energy $E^{*}$ is calculated by convolution over the active molecular and surface energy distributions for the experiment of interest,

$$
f\left(E^{*}\right)=\int_{0}^{E^{*}} f_{n}\left(E_{n}\right) \int_{0}^{E^{*}-E_{n}} f_{s}\left(E_{s}\right) f_{v}\left(\eta_{v}^{-1}\left(E^{*}-E_{n}-E_{s}\right)\right) d E_{s} d E_{n}
$$

Ultimately, 3 parameters are required for the d-PMMT model, $\left\{E_{0}, s, \eta_{v}\right\}$, and these were determined by fitting to a limited set of nonequilibrium supersonic molecular beam experiments performed by Luntz and Bethune $^{30}$ at two different nozzle temperatures. Experimental sticking coefficients are calculated as the average of $S\left(E^{*}\right)$ over $f\left(E^{*}\right)$ as in Eq. (3). Figure 3.2 provides an example of $S\left(E^{*}\right)$ and shows how an $f\left(E^{*}\right)$ is assembled from its constituent experimental distributions ${ }^{10}$ within the d-PMMT model. The overall PC energy distributions for a non-equilibrium eigenstate-resolved supersonic molecular beam experiment and for a thermal dissociative sticking experiment at the same surface temperature are also contrasted in Fig. 3.2. The breadths of the $f\left(E^{*}\right)$ s for both kinds of experiment are similar and dominated by the breadth of the surface oscillator (phonon) distribution.

\subsection{Tunneling}

To account for tunneling through the barrier to dissociative chemisorption, the RRKM rate constant for dissociative chemisorption was written in its generalized form as, ${ }^{46}$

$$
\begin{aligned}
k_{R}(E) & =\frac{W_{R}(E)}{h \rho(E)}=\int_{0}^{E} p\left(\varepsilon_{t}\right) k_{R}\left(E, \varepsilon_{t}\right) d \varepsilon_{t} \\
& =\frac{1}{h \rho(E)} \int_{0}^{E} p\left(\varepsilon_{t}\right) \rho_{R}\left(E-\varepsilon_{t}\right) d \varepsilon_{t}
\end{aligned}
$$


where $E=E^{*}+Z_{R e}$ is the classical energy above the electronic potential energy surface whose zero is set by the well-separated reactants at $\mathrm{T}=0 \mathrm{~K}, \varepsilon_{t}$ is the translational energy along the reaction coordinate leading to separated products, $p\left(\varepsilon_{t}\right)$ is the tunneling probability, and $\rho_{R}\left(E-\varepsilon_{t}\right)$ is the density of states, excluding the reaction coordinate mode, of the reactive transition state evaluated at the energy available to populate vibrational states of the transition state complex when tunneling occurs at $\varepsilon_{t} \cdot{ }^{40,47}$ The barrier to chemisorption was approximated by a $1 \mathrm{D}$ Eckart potential ${ }^{48,49}$ whose height was $E_{c}=E_{0}-\left(Z_{T S}-Z_{R e}\right)$ while the curvature, $v_{r x n}=953 i \mathrm{~cm}^{-1}$, was fixed by GGA-DFT ${ }^{39}$ and the activation barrier for associative desorption of methane, ${ }^{50-52} E_{a}=71 \mathrm{~kJ} / \mathrm{mol}$, was ultimately use to help define the exothermicity, $\Delta_{r x n} H=E_{a}\left(\mathrm{CH}_{4} \rightarrow \mathrm{CH}_{3}+\mathrm{H}\right)-E_{a}\left(\mathrm{CH}_{3}+\mathrm{H} \rightarrow \mathrm{CH}_{4}\right)=-6 \mathrm{~kJ} / \mathrm{mol}$. For the range of $E_{o}$ values explored, the overall reaction was always considered to be exothermic. The analytic expression $^{48}$ for the tunneling probability through a 1D Eckart barrier relates to $\varepsilon_{t}$ above the classical electronic potential evaluated for the well-separated reactants, without regard to zeropoint energies, and so the sum of states for the reactive transition state referenced to the $E^{*}$ energy scale can be written as,

$$
W_{R}\left(E^{*}\right)=\int_{0}^{E^{*}+Z_{R e}} p\left(\varepsilon_{t}\right) \rho_{R}\left(E^{*}+Z_{R e}-\varepsilon_{t}\right) d \varepsilon_{t}
$$

where the argument of $\rho_{R}$ is the energy available to populate vibrational states of the transition state complex, taking in to account their vibrational zero-point energy. In the absence of tunneling, 


$$
p\left(\varepsilon_{t}\right) \begin{cases}0, & E<E_{c l}+Z_{T S} \\ 1, & E \geq E_{c l}+Z_{T S}\end{cases}
$$

and given $\varepsilon_{t}=\varepsilon_{t}^{*}+Z_{R e}$, Eq.(3.13) reduces to the conventional non-tunneling expression,

$$
\begin{aligned}
W_{R}\left(E^{*}\right) & =\int_{E_{c l}+Z_{T S}}^{E^{*}+Z_{R e}} \rho_{R}\left(E^{*}+Z_{R e}-\varepsilon_{t}\right) d \varepsilon_{t} \\
& =\int_{E_{0}}^{E^{*}} \rho_{R}\left(E^{*}-\varepsilon_{t}^{*}\right) d \varepsilon_{t}^{*}=\int_{0}^{E^{*}-E_{0}} \rho_{R}\left(E^{*}-E_{0}-\varepsilon_{t}^{*}\right) d \varepsilon_{t}^{*} \\
& =W_{R}^{\dagger}\left(E^{*}-E_{0}\right)=\int_{0}^{E^{\dagger}} \rho_{R}^{\dagger}\left(E^{\dagger}\right) d E^{\dagger}
\end{aligned}
$$

where the arguments of the daggered quantities are referenced to the energy of the transition state above its zero-point energy, e.g., $E^{\dagger}=E^{*}-E_{0}=E-E_{c}-Z_{T S}$. To account for tunneling, it was operationally convenient to calculate the convolution of Eq. (3.13) as,

$$
W_{R}\left(E^{*}\right)=\int_{0}^{E^{*}+Z_{R e}} p\left(\varepsilon_{t}\right) \rho_{R}^{\dagger}\left(E^{*}+Z_{R e}-Z_{T S}-\varepsilon_{t}\right) d \varepsilon_{t}
$$

using the Beyer-Swinehart algorithm for $\rho_{R}^{\dagger}\left(E^{\dagger}\right)$. Tunneling was not relevant to desorption because the desorption barrier is infinitely thick and approached asymptotically, so that $W_{D}\left(E^{*}\right)=W_{D}^{\dagger}\left(E^{*}\right)$. With sums of states calculated in these ways, $S\left(E^{*}\right)$ was evaluated. In particular simulations of experimental dissociative sticking coefficients where it was interesting to exclude tunneling pathways (i.e., by implementing the Eq.(3.14) Heaviside conditions on $\left.p\left(\varepsilon_{t}\right)\right), S\left(E^{*}\right)$ was reduced to $W_{R}^{\dagger}\left(E^{*}-E_{0}\right) /\left[W_{R}^{\dagger}\left(E^{*}-E_{0}\right)+W_{D}^{\dagger}\left(E^{*}\right)\right]$.

\subsection{Results and Discussion}

\subsection{A. Vibrational Efficacy}


Figure 3.3 compares the dissociative sticking coefficients measured in supersonic molecular beam experiments ${ }^{21,30,38,53}$ over a range of experimental conditions with the values simulated using the s-PPMT and d-PMMT models. Versions of each kind of model were examined under the assumption that rotation was a spectator to dissociative chemisorption and also under the assumption that rotational energy was fully active in dissociative chemisorption. In Figs. 3.3-3.5 we present the model results that provided the best agreement between theory and experiments within their class: the s-PMMT model with active rotations and the d-PMMT model with rotation as a spectator. The parameters for all these models were determined by minimizing the ARD to simulations of the limited subset of the Luntz ${ }^{30}$ supersonic molecular beam data that appears in Figs 3.3(a) and (b): experiments at $T_{s}=800 \mathrm{~K}$ that scanned the $\left\langle E_{n}\right\rangle$ of the incident molecules at the two different nozzle temperatures of $T_{N}=680 \mathrm{~K}$ and $300 \mathrm{~K}$. In consequence, the single $\eta_{v}$ parameter of the d-PMMT model was fixed by a thermal averaging over the likely mode-specific ${ }^{18} \eta_{v}$ s of the active vibrational modes at these two $T_{N}$ values.

The s-PMMT model with $\left\{E_{0}=47.3 \mathrm{~kJ} / \mathrm{mol}, s=3\right\}$ and active rotations has the most difficulty in reproducing the Luntz ${ }^{30,53}$ data at $T_{N}=680 \mathrm{~K}$ in Figs $3.3(\mathrm{a})$, (c) at low $E_{n}$ where it consistently overpredicts the dissociative sticking coefficients. For the Fig. 3.3(a) Luntz data, the s-PMMT model also overpredicts the drop in dissociative sticking coefficient when the nozzle temperature was reduced from $T_{N}=680 \mathrm{~K}$ to $300 \mathrm{~K}$. This behavior is consistent with the model's vibrational efficacy relative to $E_{n}$ being too high at $\eta_{v}=1$. The model does reasonably well at predicting the $\mathrm{Beck}^{21}$ thermal nozzle experiments but consistently underpredicts the Madix ${ }^{38}$ experiments. 
The d-PMMT model with $\left\{E_{0}=58.9 \mathrm{~kJ} / \mathrm{mol}, s=2, \eta_{v}=0.40\right\}$ and spectator rotations quantitatively reproduces the Luntz and Beck experiments of Figure 3.3 but the Madix experiments are again underpredicted. It's not exactly clear why the Madix determined sticking coefficients are higher than the predictions but those experiments are believed to have been performed on a $\mathrm{Pt}(111)$ surface with a relatively high surface defect density ${ }^{54}$ and because $\mathrm{CH}_{4}$ dissociative chemisorption is a structure sensitive reaction the defects might have contributed to higher measured sticking coefficients.

Beck's experimental measurements of $\mathrm{CH}_{4} / \mathrm{Pt}(111)$ dissociative sticking coefficients for a thermal nozzle and $\left(2 v_{3}, J=2\right)$ state-resolved supersonic molecular beams are presented in Figure 3.4 along with theoretical simulations of the experiments. The s-PMMT model grossly overpredicts the $\left(2 v_{3}, J=2\right)$ state-resolved sticking coefficients whereas the d-PMMTmodel reproduces the experimental measurements extraordinarily well. Beck used his experimental data to directly measure the mode-specific vibrational efficacy relative to $E_{n}$ according to,

$$
\left.\eta_{v} \equiv \frac{\Delta E_{n}}{\Delta E_{v}}\right|_{\Delta S_{v}}
$$

where $\Delta S_{v}$ is the change in sticking coefficient in going from the ground state beam to the vibrationally excited beam at some particular $E_{n}, \Delta E_{v}$ is the vibrational energy difference between the ground state and vibrationally excited beams, and $\Delta E_{n}$ is the change in $E_{n}$ required to gain the same $\Delta S_{v}$ change along the ground state dissociative sticking coefficient curve. Beck found that $\Delta E_{v}=72 \mathrm{~kJ} / \mathrm{mol}$ of $2 v_{3}$ vibrational excitation produces a change in dissociative sticking coefficient that is equivalent to a change of only $\Delta E_{n}=27.4 \mathrm{~kJ} / \mathrm{mol}$ for the ground state 
molecules and so $\eta_{2 v_{3}}=0.38$. Given that $\eta_{v}$ values for methane dissociative chemisorption on other surfaces can be vibrational mode-specific, ${ }^{18}$ the remarkable agreement of the d-PMMT model with the Fig. 3.4 experiments could be fortuitous. On the other hand, the d-PMMT parameters were fixed by ARD minimization to Luntz's Fig. 3(b) beam experiments at $T_{N}=680$ $\mathrm{K}$ and $300 \mathrm{~K}$ and so the resulting $\eta_{v}=0.40$ parameter reflects a thermal average over the vibrational modes important to the dissociative sticking in those heated nozzle experiments. It is not so surprising that Beck's state-resolved $\eta_{2 v_{3}}=0.38$ value is close to the thermally stateaveraged $\eta_{v}$ value. Unfortunately, the only state-resolved experimental measurements of $\eta_{v}$ for the $\mathrm{CH}_{4} / \mathrm{Pt}(111)$ system are for the $2 v_{3}$ mode. $^{21} 55$

\subsection{B. Rotation as a Spectator}

The asymmetry of the rotational state populations in supersonic and effusive ${ }^{15,} 16$ molecular beam experiments measuring $\mathrm{CH}_{4} / \mathrm{Pt}(111)$ dissociative sticking coefficients provides an opportunity to investigate the role of rotation in the gas-surface reaction dynamics. Rotational cooling in $\mathrm{CH}_{4}$ supersonic expansions leaves supersonic beam molecules with a rotational temperature estimated as $T_{r}=0.1 T_{N}$ whereas in an effusive beam the molecular rotational temperature is fixed by the nozzle temperature, $T_{r}=T_{N}\left(=T_{g}\right)$, which also fixes the gas temperature. Vibrational cooling of $\mathrm{CH}_{4}$ in supersonic expansions is inefficient and so for both supersonic and effusive beams the molecular vibrational temperature should be given by the nozzle temperature, $T_{v}=T_{N}$.

Figure 3.5 compares the dissociative sticking coefficients measured in effusive molecular beam experiments ${ }^{15,16}$ with the values simulated using the s-PPMT and d-PMMT models. The effusive beam was incident along the surface normal in these experiments and the Fig. 3.5 legend 
notes the gas temperatures of the beam. The " $T_{g}=T_{s}$ ambient" entry in the legend is for simulations of the thermal equilibrium dissociative sticking coefficient appropriate to an ambient thermal gas above the surface.

The s-PMMT model overpredicts the effusive beam dissociative sticking coefficients by a factor of $\sim 6$ and the average relative discrepancy, ARD, between theory and experiment is $648 \%$. The d-PMMT model simulates the effusive beam data much better and achieves an ARD of $47 \%$. Not all of the overprediction of the s-PMMT model can be attributed to having active rotations because the model's vibrational efficacy at the statistical value of $\eta_{v}=1$ is high in comparison to experiments. To isolate the effect of rotation, we should compare the effusive beam predictions of d-PMMT models with active rotation and with the rotation as a spectator. As for the other models, the $\left\{E_{0}=42.9 \mathrm{~kJ} / \mathrm{mol}, s=2, \eta_{v}=0.40\right\}$ parameters of the d-PMMT model with active rotation were optimized to the Luntz supersonic molecular beam data of Fig. 3.3(a),(b). It turns out that the d-PMMT model with active rotations overpredicts the effusive beam data and yields an ARD of 338\%. Consequently, it seems that taking rotation as a spectator to $\mathrm{CH}_{4} / \mathrm{Pt}(111)$ dissociative chemisorption is a good approximation and provides the only way to reconcile the observed supersonic and effusive beam reactivity amongst the kinetic models considered.

Fractional energy uptakes for dissociative chemisorption are defined as the mean energy derived from a particular degree of freedom for those PCs which successfully react divided by the total mean energy for all PCs that successfully react, $f_{i}=\left\langle E_{i}\right\rangle_{R} /\left\langle E^{*}\right\rangle_{R}$. The fractional energy uptakes provide a useful means to identify from which degrees of freedom energy is drawn to overcome the activation barrier for methane dissociative chemisorption. Fig. 3.6 shows the statistical (a \& c) and dynamical (b \& d) PMMT fractional energy uptakes as a functional of 
incident normal translational energy for successfully reacting PCs under supersonic beam (a \& b) and eigenstate resolved ( $c \& d$ ) experimental conditions. In the ground state, both s- and dPMMT calculations found that at low translational energy, $E_{n}=10 \mathrm{~kJ} / \mathrm{mol}$, the surface provides nearly all of the energy to those PCs that successfully react. The relative contribution of the surface was found to decrease in a fairly linear manner such that for the high energy molecules at high translational energy the role of the surface has shrunk from $\sim 80 \%$ to $20 \%$. At all translational energies, rotation and vibration combined supply less than 5\% of the total exchangeable energy used to surmount the barrier to dissociation. For the excited, $2 v_{3}, J=2$, case, vibration was found to be of primary importance in reactivity for the s-PMMT fractional energy uptakes with normal translational energies less than $65 \mathrm{~kJ} / \mathrm{mol}$, but only supplies $30-40 \%$ of the exchangeable energy for uptakes calculated from d-PMMT modeling.

Figure 3.7 presents a compilation of the ARDs for the 3 theoretical models discussed above against the different kinds of experimental data. Given that all the models were optimized to the same Luntz supersonic molecular beam data there is not too much dispersion in ARD values for the Beck and Luntz supersonic beam experiments. All the models agree that the Madix data is high compared to the Luntz and Beck thermal nozzle experiments. Only the d-PMMT models fare well with the Beck $2 v_{3}$ experiments. Cushing's effusive beam experiments resolve with good dispersion that the d-PMMT model with rotation as a spectator is best. The overall ARDs across all the experiments were 451\%, 95\%, and 43\% for the s-PMMT model with active rotations, d-PMMT model with active rotation, and d-PMMT model with rotation as a spectator, respectively. The exponential nature of the variability in d-PMMT calculated dissociative sticking measurements, and thus exponential difference in the ARD, as a function of efficacy of vibration and threshold barrier height are shown in Figure 3.8(a) and 3.8(b) respectively, speaking to the strength of the values found. 
The angular variation of amplitude scaled d-PMMT calculations is compared to experimental thermal dissociative sticking coefficient ${ }^{56}, S(700 \mathrm{~K}, \vartheta)$, in Figure 3.9. Error bars of one standard deviation are indicated for a few representative experimental data points. Table 3.1 catalogs the different $S_{0}$ and $n$ values used to fit both the d-PMMT and experimental predictions for $S(700 \mathrm{~K}, \vartheta)$ using $S_{0} \cos ^{n} \vartheta$. Rotational efficacy, $\eta_{r}$, values of 1 and 0 in Table 3.1 indicate where molecular rotations were considered to be fully active or spectator degree of respectfully. The dr-PMMT model is dynamically biased vibrationally but treats rotation as a fully active degree of freedom. The sharpness of the angular variation of $S(T, \vartheta)$ increases with $E_{0}$ but also depends largely on the relative importance of $E_{n}$ in dictating whether the condition $E^{*} \geq E_{0}$ can be met at the different incidence angles, $\vartheta$. For example, the less probable assembly of PCs with $E^{*} \geq E_{0}$ under the dynamical restrictions of the d-PMMT model (i.e., $\eta_{v}=0.4, \eta_{r}=0$ ) as compared to assembly for the s-PMMT model, enhances the relative importance of $E_{n}$ in surmounting the activation barrier for reaction under thermal equilibrium conditions. Generally, the more dynamically constrained PMMT model will always calculate the sharper angular distribution when $s$ and $E_{0}$, are held constant across different models. The amplitude scaled d-PMMT prediction varies as $\cos ^{12.1} \vartheta$, with a scaled ARD of $12.1 \%$, and stays within the limits of the experimental error bars. The dr-PMMT and s-PMMT angular sticking variations are much broader than the experimental sticking and yield considerably higher scaled ARDs. Table 3.1 shows that the d-PMMT model performs considerably better than the other two models in its prediction of the absolute sticking. The optimal scaling divisors noted in Table 3.1 indicate that the d-PMMT model underpredicts the experimental sticking by $37 \%$ whereas the drPMMT and s-PMMT models overpredict by $340 \%$ and $710 \%$, respectively. A one-dimensional van Willigen model $^{57}$ of the activated dissociative chemisorption (requiring $E_{n} \geq E_{0}$ to react) 
with $E_{0}=43 \mathrm{~kJ} / \mathrm{mol}$ yields $S(700 \mathrm{~K}, \vartheta)=5.2 \times 10^{-3} \cos ^{14.5} \vartheta$ which recovers the angular variation of the sticking but severely overestimates its magnitude by $6412 \%$. Overall, the agreement between the d-PMMT predictions and experimental points based on parameters derived from the translationally hyperthemal, but rotationally cold, supersonic molecular beam experiments is reasonably good, bolstering the argument for approximating rotation to be a spectator degree of freedom in the activated dissociative sticking of methane on $\operatorname{Pt}(111)$. Accordingly, the other two PMMT models with $\eta_{r}=1$ overpredict the dissociative sticking coefficient because the enhanced rotational energy in the effusive, as compared to supersonic, molecular beam experiments is incorrectly assigned as active exchangeable energy capable of helping to surmount the activation barrier to dissociative chemisorption.

\subsection{Associative Desorption}

Figure 3.10 shows the PMMT detailed balance predictions for the associative desorption of $\mathrm{CH}_{3}+\mathrm{H} \rightarrow \mathrm{CH}_{4}$ from $\mathrm{Pt}(111)$ compared to the Harrison ${ }^{58}$ and Watanabe ${ }^{59}$ experimental results. The d-PMMT predicts a temperature dependent angular distribution in associative desorption that broadens as the temperature increases. At the $240 \mathrm{~K}$ surface temperature of the Harrison experiment the d-PMMT predicts a $\cos ^{20} \vartheta$ angular distribution in associative desorption, broader than the experimentally observed $\cos ^{37} \vartheta$ distribution. The d-PMMT distribution broadens to $\cos ^{17.7} \vartheta$ at the $395 \mathrm{~K}$ surface temperature of the Watanabe experiment, still remaining broader than the $\cos ^{31} \vartheta$ distribution observed experimentally. The difference between the d-PMMT predictions and the experimental distributions may be attributable to the

close to saturation coverage of hydrogen atoms and methyl radicals, as well as bromine atoms in the Harrison experiment. 
The issue of reactant decomposition may degrade the applicability of detailed balance for dissociative chemisorption systems with polyatomic chemisorbed product species, for the purposes of d-PMMT simulations we simply assume that detailed balance is applicable to the $\mathrm{CH}_{3(c)}+\mathrm{H}_{(c)} \rightarrow \mathrm{CH}_{4(g)}$ equilibrium on $\mathrm{Pt}(111)$. If methyl decomposition is not negligible, then the manner in which detailed balance is used here cannot relate dissociative chemisorption to associative desorption. Campbell's recent studies on methyl iodide decomposition found the reaction pathway from adsorbed methyl to methylidyne and coadsorbed hydrogen atoms, $\mathrm{CH}_{3(c)} \rightleftarrows \mathrm{CH}_{(c)}+2 \mathrm{H}_{(c)}$, at temperatures up to $320 \mathrm{~K}$ and in the low coverage limit, $\vartheta<0.1 M L$, has an equilibrium constant shifted far to the left thereby stabilizing methyl coverages and bolstering the applicability of detailed balance applied to methyl radical hydrogenation. ${ }^{60}$

Figure 3.11 compares experimental $\mathrm{CH}_{4}$ associative desorption translational energy distributions with PMMT simulations with increasing dynamical biasing. As the energy available in rotation and vibration diminishes, the mean translational energy of the molecules which successfully react increases from 17.4 to $33.8 \mathrm{~kJ} / \mathrm{mol}$, tending towards to the experimental value of $40.5 \mathrm{~kJ} / \mathrm{mol}$. While the d-PMMT model best replicates the experimental $\mathrm{P}\left(\mathrm{E}_{\mathrm{t}}\right)$, the model overpredicts the molecular probability at low $\mathrm{E}_{\mathrm{t}}$ and underpredicts the tail at high $\mathrm{E}_{\mathrm{t}}$. In the time of flight spectra, the low $\mathrm{E}_{\mathrm{t}}$ tail of the $\mathrm{P}\left(\mathrm{E}_{\mathrm{t}}\right)$ distribution is harder to detect using the number density detecting mass spectrometer than are more energetic molecules (i.e. $n(t) \propto\left(1 / t^{4}\right) P\left(E_{t}\right)$ where $E_{t}=\frac{1}{2} m\left(\frac{s}{t}\right)^{2}$ is the translational energy of the desorbing molecules, s is the distance between the mass spectrometer ionizer and crystal, and $\mathrm{t}$ is the time required to reach the ionizer). 
A useful method of investigating the impact of normal translational energy on the dissociative sticking of a thermal system is to divide the associative desorption product energy distribution by a Maxwell Boltzmann distribution to recover a relative dissociative sticking coefficient. Detailed balance requires the associative desorption flux, $D_{0}$, to balance the dissociative chemisorption flux, $S F_{o}$, at thermal equilibrium where $S$ and $F_{o}$ are the thermal dissociative sticking coefficient and molecular flux, respectively.

$$
D_{o}=S F_{o}
$$

and the microscopically detailed version of Eq. 3.18 is,

$$
D\left(E_{t}, \vartheta ; T\right)=S\left(E_{t}, \vartheta ; T\right) f_{M B}\left(E_{t}, \vartheta ; T\right)
$$

where $f_{M B}$ is flux-weighted Maxwell-Boltzmann thermal translational energy distribution

$$
f_{M B}\left(E_{t}, \vartheta ; T\right)=\frac{\cos (\vartheta)}{\pi} \frac{E_{t}}{\left(k_{b} T\right)^{2}} e^{-E_{t} / k_{b} T}
$$

By detailed balance, the translational energy-resolved reactive flux, desorbing or dissociatively chemisorbing, is proportional to the associative desorption product translational energy distribution, $\mathrm{P}\left(\mathrm{E}_{\mathrm{t}}\right)$ measured experimentally,

$$
D\left(E_{t}, \vartheta ; T\right)=S\left(E_{t}, \vartheta ; T\right) f_{M B}\left(E_{t}, \vartheta ; T\right) \propto P_{t}\left(E_{t}\right)
$$

such that the relative thermal dissociative sticking as a function of translational energy can be determined as

$$
S_{r e l}\left(E_{t}\right)=\frac{P_{t}\left(E_{t}\right)}{f_{M B}\left(E_{t}, \vartheta ; T\right)} \propto S\left(E_{t}, \vartheta ; T\right)
$$


Figure 3.12 compares d-PMMT thermal dissociative sticking coefficients for methane on $\mathrm{Pt}(111)$ to points derived from time of flight experiments as a function of normal translational energy. The relative dissociative sticking curves derived in this manner have been normalized such that the normal translational energy integrated sticking, $S\left(\vartheta=0^{\circ} ; T\right)$, across all translational energies is equivalent to d-PMMT predictions. Experimental translational energies above $100 \mathrm{~kJ} / \mathrm{mol}$ were considered to be at the level of noise and were not included in the normalization.

Figure 3.13 compares mean energies of successfully reacting molecules at $395 \mathrm{~K}$ to experimental mean desorbing energies calculated from associative desorption of methane on $\mathrm{Pt}(111)$. The mean energies are calculated as,

$$
\left\langle E_{i}(\vartheta)\right\rangle=\frac{\int_{0}^{\infty} E_{i} \xi\left(E_{i}, \vartheta\right) d E_{i}}{\int_{0}^{\infty} \xi\left(E_{i}, \vartheta\right) d E_{i}}
$$

where $i=v, t, s$. The reactive mean normal translational energy is simply $\left\langle E_{n}(\vartheta)\right\rangle=\left\langle E_{t}(\vartheta)\right\rangle \cos ^{2} \vartheta$. Vibration was found to supply a negligible amount of energy across all angles. The surface and normal translation initially supply energy equally at zero degrees, with the surface providing an increasing contribution of the total energy as the incident angle increases. Experimentally derived mean translational energies show negligible variation up to the limit of detection, $\vartheta \approx 25^{\circ}$, similar to those derived from d-PMMT calculations, which drop by $\sim 10 \mathrm{~kJ} / \mathrm{mol}$, equivalent to a $28 \%$ change, over the same range.

\subsection{Reactivity in Supersonic Beam Experiments}


It is worth revisiting the Luntz supersonic molecular beam data of Fig. 3.3 to construct Arrhenius plots of the dissociative sticking coefficients versus $1000 / T_{n}$ at fixed values of $\left\langle E_{n}\right\rangle$ and $T_{s}$. Figure 3.14 plots the Luntz data in this manner along with simulations from the usual sPMMT and d-PMMT models. Given that molecular rotational energy is effectively frozen out in the supersonic expansion, the Fig. 3.14 data reports simply on the effect of the incident molecules' vibrational temperature on the gas-surface reactivity. Noteworthy is the very shallow response of the dissociative sticking coefficient to variation in $1000 / T_{n}$ according to experiment and the d-PMMT model. The s-PMMT model shows a much more pronounced response similar to that observed experimentally in Chorkendorrf's laboratory for dissociative chemisorption of $\mathrm{CH}_{4} / \mathrm{Ni}(100) .{ }^{61}$ The PC-MURT/s-PMMT model ${ }^{10,62}$ was able to simulate the Chorkendorrf $\mathrm{CH}_{4} / \mathrm{Ni}(100)$ supersonic molecular beam experiments quite well and so for that system $\eta_{v}$ should be closer to 1 . The characterization of the $\mathrm{CH}_{4} / \mathrm{Pt}(111)$ transition state as early $\left(\eta_{v}=0.40\right)$ and the $\mathrm{CH}_{4} / \mathrm{Ni}(100)$ transition state as later $\left(\eta_{v} \sim 1\right)$ is in good accord with expectations based on Jackson's GGA-DFT calculations which found the $\mathrm{CH}_{4} / \mathrm{Ni}(100)$ transition state occurs closer to the surface plane, $\Delta Z_{C}^{\dagger}=0.19 \AA$, and with larger $\mathrm{H}-\mathrm{CH}_{3}$ bond extension, $\Delta r^{\dagger}=0.12 \AA$, than for the $\mathrm{CH}_{4} / \mathrm{Pt}(111)$ transition state. Important to point out is that thermal nozzle supersonic molecular beam data of the kind plotted in Figure 3.14 are especially valuable for determination of thermally-averaged values of the vibrational efficacy and a range of $\eta_{v}$-dependent experimental behavior ${ }^{30,53,61}$ has already been observed.

Figure 3.15 provides an analysis of Beck's thermal nozzle and $\left(2 v_{3}, J=2\right)$ stateresolved supersonic molecular beam experiments in the context of the d-PMMT model (see also Fig. 3.4). The mean normal translational energy, $\left\langle E_{n}\right\rangle$, for the thermal nozzle beam experiments 
is shown along the lower horizontal-axis whereas the $\Delta E_{n}=28.8 \mathrm{~kJ} / \mathrm{mol}$ offset, upper horizontalaxis gives $\left\langle E_{n, 2 v_{3}}\right\rangle$ for the state-resolved experiments. Plotted in this way, the mean exchangeable energy, $\left\langle E^{*}=E_{n}+\eta_{v} E_{v}+E_{s}\right\rangle$, of the PCs formed is seen to increase in lock step for the two kinds of experiments because the vibrational excitation of $\Delta E_{v}=72 \mathrm{~kJ} / \mathrm{mol}$ is equivalent to a shift of $\Delta E_{n}=\eta_{v} \Delta E_{v}=28.8 \mathrm{~kJ} / \mathrm{mol}$ for the molecules in the thermal nozzle beam which are overwhelmingly in their ground vibrational state. With the offset $\left\langle E_{n, 2 v_{3}}\right\rangle$ scale, both kinds of experiments follow the same dissociative sticking coefficient curve. To assess what kinds of energy are being used to overcome the activation barrier for reaction in these experiments it is useful to calculate ${ }^{9}$ the mean exchangeable energy of the PCs formed that successfully react, $\left\langle E^{*}\right\rangle_{R}$, and the mean energies, $\left\langle E_{j}\right\rangle_{R}$, that derived from the $j^{\text {th }}$ degrees of freedom for the PCs that successfully react. Given that for a particular experiment, $\left\langle E^{*}\right\rangle_{R}=\left\langle E_{n}\right\rangle_{R}+\eta_{v}\left\langle E_{v}\right\rangle_{R}+\left\langle E_{s}\right\rangle_{R}$ it is convenient to define fractional exchangeable energy uptakes from the $j^{\text {th }}$ degrees of freedom as $f_{j}=\left\langle E_{j}\right\rangle_{R} /\left\langle E^{*}\right\rangle_{R}$ for $j \neq v$ and $f_{v}=\eta_{v}\left\langle E_{j}\right\rangle_{R} /\left\langle E^{*}\right\rangle_{R}$. Figure 14 shows that at low normal translational energies the energy derived from surface phonons, $\left\langle E_{s}\right\rangle_{R}$, can play the key role in facilitating reaction. For the thermal nozzle supersonic beam experiments the fractional exchangeable energy uptake from the surface, $f_{s}$, is calculated to be greater than $50 \%$ for $\left\langle E_{n}\right\rangle \leq 35 \mathrm{~kJ} / \mathrm{mol}$. Comparatively, for the $\left(2 v_{3}, J=2\right)$ state-resolved supersonic molecular beam experiments with $\left\langle E_{n, 2 v_{3}}\right\rangle \leq 15 \mathrm{~kJ} / \mathrm{mol}$, the surface phonons still provide the largest fraction of the exchangeable energy required to overcome the activation barrier with $f_{s}>38 \%$. 


\subsection{E. Effective Activation Energies}

For nonequilibrium molecular beam experiments, such as those of Figs. 3.3, 3.4, and 3.5, it is often possible to determine "effective activation energies" of a generalized ${ }^{9} \operatorname{Tolman}^{63}$ form,

$$
\begin{aligned}
\text { "E } E_{a}\left(T_{j}\right) " & =-k_{B} \partial \ln S\left(T_{j}\right) / \partial T_{j}^{-1}=k_{B} T_{j}^{2} \partial \ln S\left(T_{j}\right) / \partial T_{j} \\
& =\left\langle E_{j}\right\rangle_{R}-\left\langle E_{j}\right\rangle
\end{aligned}
$$

where $\left\langle E_{j}\right\rangle_{R}$ and $\left\langle E_{j}\right\rangle$ are the mean energies derived from the $j^{\text {th }}$ degrees of freedom for the successfully reacting gas-surface collision complexes (i.e., PCs) formed, and for all the gassurface collision complexes formed, respectively, and $T_{j}$ is the temperature of the energy distribution for the $j^{\text {th }}$ degrees of freedom. Following Tolman, ${ }^{9,63}$ this relation requires only that it be possible to write the dissociative sticking coefficient as an average of state-specific dissociative sticking coefficients over the incident gas, and surface, state distribution functions defining the experimental conditions. For example,

$$
S=\sum_{E_{v}, E_{s}}\left\{\prod_{i=v, s} f_{i}\left(E_{i}, T_{i}\right)\right\} \int_{0}^{\infty} S\left(E_{t}, E_{v}, E_{s} ; \vartheta, \phi\right) f_{t}\left(E_{t}, T_{t} ; \vartheta, \phi\right) d E_{t}
$$

where $f_{t}\left(E_{t}, T_{t} ; \vartheta, \phi\right)$ is the flux weighted translational energy distribution for the molecules incident on the surface at angle $(\vartheta, \phi)$ and the other distribution functions have the form,

$$
f_{i}\left(E_{i}, T_{i}\right)=\frac{g_{i}}{q\left(T_{i}\right)} \exp \left(-\frac{E_{i}}{k_{B} T_{i}}\right)
$$

where $g_{i}$ is the degeneracy of energy level $E_{i}$ and $q\left(T_{i}\right)$ is the partition function for the $i^{\text {th }}$ degrees of freedom prepared at temperature $T_{i}$. 
For an experiment prepared with a well-defined vibrational temperature, the active exchangeable energy available in vibration, $E_{v}^{*}=\eta_{v} E_{v}$, is governed by the energy distribution

$$
f_{v}\left(E_{v}^{*}\right)=\frac{\rho_{v}\left(E_{v}^{*} / \eta_{v}\right)}{\eta_{v} Q_{v}\left(T_{v}\right)} \exp \left(-\frac{E_{v}^{*}}{\eta_{v} k_{b} T_{v}}\right)
$$

The vibrational temperature dependent experimental sticking is formed as the average microcanonical sticking coefficient over the probability distribution of forming a precursor complex with exchangeable energy, $E^{*}$, for the particular experimental conditions at hand,

$$
\begin{aligned}
& S=\int_{0}^{E^{*}} S\left(E^{*}\right) f\left(E^{*}\right) d E^{*} \\
& =\frac{1}{\eta_{v} Q_{v}\left(T_{v}\right)} \int_{0}^{\infty} S\left(E^{*}\right) \int_{0}^{E^{*}} f_{s t}\left(E^{*}-\frac{E_{v}^{*}}{\eta_{v}}\right) \times \rho_{v}\left(E_{v}^{*} / \eta_{v}\right) \exp \left(-\frac{E_{v}^{*}}{\eta_{v} k_{b} T_{v}}\right) d E_{v}^{*} d E^{*}
\end{aligned}
$$

where $f_{s t}$ is the convolution of the surface and normal translation degrees of freedom,

$$
f_{s t}\left(E^{*}\right)=\int_{0}^{E^{*}} f_{t}\left(E_{t}\right) \times f_{s}\left(E^{*}-E_{t}\right) d E_{t}
$$

Differentiating Eq. 3.28 according to Eq 3.24 with respect to $T_{v}$ yields,

$$
" E_{a}\left(T_{v}\right) "=k_{b} T_{v}^{2} \frac{\partial\left(\ln S\left(T_{v}\right)\right)}{\partial T_{v}}
$$




$$
" E_{a}\left(T_{v}\right) "=k_{b} T_{v}^{2}\left(\begin{array}{l}
\frac{1}{\eta_{v} k_{b} T_{v}^{2}} \frac{\left.\int_{0}^{\infty} S\left(E^{*}\right) \int_{0}^{E^{*}} f_{s t}\left(E^{*}-\frac{E_{v}^{*}}{\eta_{v}}\right) \times \rho_{v}\left(E_{v}^{*} / \eta_{v}\right) E_{v}^{*} \exp \left(-\frac{E_{v}^{*}}{\eta_{v} k_{b} T_{v}}\right) d E_{v}^{*} d E^{*}\right) \int_{0}^{E^{*}} f_{s t}\left(E^{*}-\frac{E_{v}^{*}}{\eta_{v}}\right) \times \rho_{v}\left(E_{v}^{*} / \eta_{v}\right) \exp \left(-\frac{E_{v}^{*}}{\eta_{v} k_{b} T_{v}}\right) d E_{v}^{*} d E^{*}}{-\frac{\partial\left(\ln Q_{v}\left(T_{v}\right)\right)}{\partial T_{v}}}
\end{array}\right)
$$

Noting the statistical mechanics relation $\left\langle E_{j}\right\rangle=k_{B} T_{j}^{2} \partial \ln q\left(T_{j}\right) / \partial T_{j}{ }^{9}$, and

$$
\begin{gathered}
\left\langle E_{v}\right\rangle=\frac{\left\langle E_{v}^{*}\right\rangle}{\eta_{v}} \\
" E_{a}\left(T_{v}\right) "=\left(\frac{1}{\eta_{v}} \frac{\left.\int_{0}^{\infty} S\left(E^{*}\right) \int_{0}^{E^{*}} f_{s t}\left(E^{*}-\frac{E_{v}^{*}}{\eta_{v}}\right) \times \rho_{v}\left(E_{v}^{*} / \eta_{v}\right) E_{v}^{*} \exp \left(-\frac{E_{v}^{*}}{\eta_{v} k_{b} T_{v}}\right) d E_{v}^{*} d E^{*}\right)}{\left.\int_{0}^{E^{*}}\right) \int_{0}^{E_{s t}}\left(E^{*}-\frac{E_{v}^{*}}{\eta_{v}}\right) \times \rho_{v}\left(E_{v}^{*} / \eta_{v}\right) \exp \left(-\frac{E_{v}^{*}}{\eta_{v} k_{b} T_{v}}\right) d E_{v}^{*} d E^{*}}-\frac{E_{v}}{\eta_{v}}\right)
\end{gathered}
$$

The first term may be recognized as $1 / \eta_{\mathrm{v}}$ times the average of the exchangeable vibrational energy over the probability distribution for those species that successfully react such that,

$$
" E_{a}\left(T_{v}\right) "=\frac{\left\langle E_{v}^{*}\left(T_{v}\right)\right\rangle_{R}-\left\langle E_{v}^{*}\left(T_{v}\right)\right\rangle}{\eta_{v}}
$$

Eq. 3.30 is broadly applicable, in the limit that the efficacy of vibration goes to 1 , the generalized ${ }^{9}$ Tolman relation of Eq. 3.24 that applies to statistically reacting systems is recovered. Eq. 3.30 can be derived similarly for experiments prepared with well-defined molecular translational, rotational, or surface temperatures (n.b., only the single temperature of interest, $T_{j}$, need be well defined to calculate " $E_{a}\left(T_{j}\right)$ "). Eq. 3.30 applies to molecular translation for effusive molecular beams but not to translation for supersonic molecular beams which have an unusual, non- 
equilibrium, and relatively narrow $f_{t}\left(E_{t}, T_{t} ; \vartheta, \phi\right)$ distribution. These results are quite general and are not constrained by any particular model, except the reactivity should depend on the precursor complex energy and not particular quantum states. The surface degrees of freedom and associated energies can be taken as any that influence the gas-surface reactivity.

Effective activation energies determined directly from the Luntz supersonic molecular beam experiments of Figure 3.3 are plotted in Figure 3.16, along with d-PMMT predictions. The $S\left(T_{s}\right)$ experimental data of Fig. 3.3(d) yield effective activation energies " $E_{a}\left(T_{s}\right)$ " that increase from $3.3 \mathrm{~kJ} / \mathrm{mol}$ to $29.4 \mathrm{~kJ} / \mathrm{mol}$ as the normal translational energy decreases from $122.5 \mathrm{~kJ} / \mathrm{mol}$ to $40.5 \mathrm{~kJ} / \mathrm{mol}$. Effective activation energies derived from varying the nozzle temperature are $" E_{a}\left(T_{N}\right) "=" E_{a}\left(T_{v}\right) "$ given that molecular rotations are frozen out by the supersonic expansion and the incident molecules' vibrational temperature is given by the nozzle temperature. Consequently, the Fig. $3.14 S\left(T_{N}\right)$ experimental data yields " $E_{a}\left(T_{v}\right)$ " values that increase from $0.83 \mathrm{~kJ} / \mathrm{mol}$ to $3.0 \mathrm{~kJ} / \mathrm{mol}$ as $\left\langle E_{n}\right\rangle$ decreases from $50 \mathrm{~kJ} / \mathrm{mol}$ to $30 \mathrm{~kJ} / \mathrm{mol}$. The relative size of these wholly experimentally derived effective activation energies establish that surface degrees of freedom are much more important than vibrational or rotational degrees of freedom in promoting gas-surface reactivity in these supersonic molecular beam experiments. Based on Eq. (3.19), $\left\langle E_{j}\right\rangle_{R}=" E_{a}\left(T_{j}\right) "+\left\langle E_{j}\right\rangle \geq " E_{a}\left(T_{j}\right) "$, so the effective activation energies provide lower bounds on the mean energies derived from the $j^{\text {th }}$ degrees of freedom for the successfully reacting gassurface collision complexes. Fig. 3.16 shows that $\mathrm{CH}_{4} / \mathrm{Pt}(111)$ supersonic beam experiments at $T_{N}=680 \mathrm{~K}$ and $T_{s}=800 \mathrm{~K}$, with $\left\langle E_{n}\right\rangle=40.5 \mathrm{~kJ} / \mathrm{mol}$, have $\left\langle E_{s}\right\rangle_{R} \geq " E_{a}\left(T_{s}\right) "=29.5 \mathrm{~kJ} / \mathrm{mol}$. Even for this markedly hyperthermal experiment, the mean energy drawn from the surface by the successfully reacting gas-surface collision complexes is at least a sizeable fraction $(\geq 73 \%)$ of 
their mean normal translational energy (n.b., $\left\langle E_{n}\right\rangle_{R} \approx\left\langle E_{n}\right\rangle$ because of the narrow $f_{t}\left(E_{t}\right)$ of the supersonic molecular beam).

The d-PMMT model reproduces the Luntz experimental observations of Fig. 3.16 quite well and predicts " $E_{a}\left(T_{s}\right) " \rightarrow 62 \mathrm{~kJ} / \mathrm{mol}$ and $" E_{a}\left(T_{N}\right) " \rightarrow 3.9 \mathrm{~kJ} / \mathrm{mol}$ as $\left\langle E_{n}\right\rangle \rightarrow 0 \mathrm{~kJ} / \mathrm{mol}$. Similar d-PMMT analysis of the Beck supersonic beam experiments in Figs. 3.6, 3.13, and 3.18 further illustrate that it is the relative availability of energy from the different degrees of freedom under the particular experimental conditions that dictates the fractional energy uptakes employed to surmount the activation barrier. Accordingly, the derivation of Eq. (3.19) from Eq. (3.20) confirms that the "E $E_{a}\left(T_{j}\right) "$, and hence the $\left\langle E_{j}\right\rangle_{R}=" E_{a}\left(T_{j}\right) "+\left\langle E_{j}\right\rangle \geq " E_{a}\left(T_{j}\right) "$, should be strongly influenced by the particular experimental conditions (i.e., the variable $\left\{T_{i}\right\}$ whereas the $S\left(E_{t}, E_{v}, E_{r}, E_{s} ; \vartheta, \phi\right)$ are fixed). Unfortunately, there are relatively few measurements of $" E_{a}\left(T_{j}\right) "$ for activated dissociative chemisorption in the literature. Noteworthy is Hodgson's experimental determination ${ }^{27}$ of $" E_{a}\left(T_{s}\right) "=52 \mathrm{~kJ} / \mathrm{mol}$ for the state resolved dissociative sticking coefficient for $\mathrm{H}_{2}(v=0, J=1)$ incident at $\left\langle E_{n}\right\rangle=4.3 \mathrm{~kJ} / \mathrm{mol}$ on a $\mathrm{Cu}(111)$ surface at $T_{s}=600$ $\mathrm{K}$, which established that $\left\langle E_{j}\right\rangle_{R} \geq 52 \mathrm{~kJ} / \mathrm{mol}$ and it is possible to extract at least $52 \mathrm{~kJ} / \mathrm{mol}$ energy from surface phonons in order to surmount the activation energy for dissociative chemisorption of $\mathrm{H}_{2}$ on $\mathrm{Cu}(111)$. This $\mathrm{H}_{2} / \mathrm{Cu}(111)$ result has been replicated by a s-PMMT model assuming rotation is a spectator ${ }^{12}$ but is also very similar to the d-PMMT model prediction for $\mathrm{CH}_{4} / \mathrm{Pt}(111)$ that $E_{a}\left(T_{s}\right) " \rightarrow 62 \mathrm{~kJ} / \mathrm{mol}$ as $\left\langle E_{n}\right\rangle \rightarrow 0 \mathrm{~kJ} / \mathrm{mol}$ in Fig. 3.13. Certainly, the " $E_{a}\left(T_{s}\right) "$ experiments alone establish that the surface can play an increasingly important, if not dominant, role in providing the energy necessary to surmount the activation barrier to dissociative 
chemisorption when the energy available from the incident molecules is low and relatively restricted.

Although both $\mathrm{H}_{2} / \mathrm{Cu}(111)$ and $\mathrm{CH}_{4} / \mathrm{Pt}(111)$ have traditionally been considered to be prototypical gas-surface reaction systems exhibiting "direct dynamics", wherein the gas-surface collision lifetime is ultrashort and energy exchange with the surface is of secondary, ${ }^{64}$ or perhaps negligible, ${ }^{65}$ importance, the $" E_{a}\left(T_{s}\right) "$ experiments make clear that molecular and surface degrees of freedom are strongly coupled on the reactive potential energy surface. For example, the $\mathrm{CH}_{4} / \mathrm{Pt}(111)$ hyperthermal supersonic molecular beam experiments at $\left\langle E_{n}\right\rangle=40.5 \mathrm{~kJ} / \mathrm{mol}$ show that at least comparable amounts of energy $(>72 \%)$ are extracted from the surface for use in surmounting the activation barrier to reaction. Accordingly, rather than treat the surface degrees of freedom as a perturbation, the PMMT models invoke sufficient collisional coupling amongst the surface and active molecular degrees of freedom to yield microcanonical randomization of a PC's active energy whilst ultrafast desorption rates at reactive energies avoids PC thermalization to the surface temperature. These few concepts suffice to build statistical models capable of closely reproducing many experimental observations on activated gas-surface reactions. ${ }^{9,12-14,36}$

\subsection{F. Reactivity Under Thermal Equilibrium Conditions}

Under the high pressure conditions of industrial catalytic processes, catalyst surfaces and the ambient gases above them are thermalized to a common temperature. The thermal dissociative sticking coefficient, $S(T)$, for $\mathrm{CH}_{4} / \mathrm{Pt}(111)$ is simulated in Fig. 3.17 according to the d-PMMT model. There is some curvature in the $S(T)$ Arrhenius plot and the local activation energy is given by the Tolman relation, ${ }^{9}, 63 E_{a}(T)=-R \partial \ln S(T) / \partial T^{-1}=\left\langle E^{*}\right\rangle_{R}-\left\langle E^{*}\right\rangle$. A simple Arrhenius fit to the dissociative sticking coefficient yields $S(T)=S_{0} \exp \left(-E_{a} / R T\right)$ where the 
pre-exponential factor is $S_{0}=0.62$ and the activation energy is $E_{a}=62.6 \mathrm{~kJ} / \mathrm{mol}$. Figure 16 also provides an accounting of the mean exchangeable energies derived from the different degrees of freedom for the successfully reacting PCs. Energy from the surface phonons, $\left\langle E_{s}\right\rangle_{R}$, is always the most significant contributor to $\left\langle E^{*}\right\rangle_{R}$.

Fig. 3.18 illustrates how the fractional energy uptakes for dissociative chemisorption of $\mathrm{CH}_{4} / \mathrm{Pt}(111)$ under thermal equilibrium conditions vary with temperature as calculated with the sPMMT and d-PMMT theoretical models. The d-PMMT model with rotation as a spectator and $\eta_{v}=0.40$ results in qualitatively different $f_{j} \mathrm{~s}$ as compared to the s-PMMT model with active rotations (our default MURT model to date $9,11,15,16,36,66$ ) although both models agree that surface phonons contribute a major fraction of the active exchangeable energy required to overcome the activation barrier. Here, the d-PMMT model contends that surface phonons, and not the incident molecules, account for the majority of the exchangeable energy used to overcome the activation barrier. For the temperature range $T \leq 1000 \mathrm{~K}$ relevant to catalytic reforming of methane, ${ }^{6} f_{s} \geq$ $56 \%, f_{n} \geq 28 \%$, and $f_{v} \leq 16 \%$ such that $f_{s}$ always exceeds the net fractional energy uptake from the gas, $f_{g} \leq 44 \%$. This is the first theoretical assessment that it is the surface phonons, and not the incident molecules, that play the dominant role in supplying the energy required to overcome the activation barrier for $\mathrm{CH}_{4} / \mathrm{Pt}(111)$ dissociative chemisorption under the thermal equilibrium conditions relevant to high pressure catalysis. Although these energy supply roles can be reversed in non-equilibrium dissociative chemisorption experiments if sufficiently energetic molecular beams are made incident on the surface (e.g., Figs. 3.7 and 3.15), under thermal equilibrium conditions, or at sufficiently low $\left\langle E_{n}\right\rangle$, surface phonons dominate the fractional energy uptake. These findings are significant because it is only relatively recently that 
mainstream theoretical work on gas-surface reaction dynamics ${ }^{22,67}$ has begun to explicitly treat surface $^{36,68,69}$ degrees of freedom and recognize their importance.

Figure 3.19 shows the angle-resolved mean reacting energies and distributions for individual degrees of freedom for successfully reacting species at $873 \mathrm{~K}$. Effective temperatures of the reacting distributions depicted in $3.19 \mathrm{~b}$, c, and $\mathrm{d}$ are shown in Table 3.2. The reacting distributions derived from s-PMMT calculations for both angle integrated and directed along the surface normal have an equivalent effective temperature across all degrees of freedom of 1150 and $1500 \mathrm{~K}$ respectively. d-PMMT derived reacting distributions have equivalent, but slightly higher, effective temperatures for translation and surface degrees of freedom under angle integrated and incidence normal to the surface conditions, 1450 and $1600 \mathrm{~K}$, respectively, with vibration in each case found to be slightly elevated. For those molecules incident nearly parallel to the surface, the effective vibrational temperature rose slightly, the normal translation component fell to approximately $0 \mathrm{~K}$, and the surface energy distribution became extremely hyperthermal. This is consistent with the d-PMMT predictions of $\left\langle E_{j}(\vartheta)\right\rangle$ of Fig 3.19(a), because with rotation as a spectator, as $\vartheta \rightarrow 89^{\circ}$ and normal translational energy goes towards zero, phonons present a more flexible reservoir of energy than do the dynamically discounted molecular vibrations for supplying the energy necessary for PCs to successfully react. For the dPMMT angle integrated and normal incidence cases, the effective vibrational temperature was found to be the highest, but vibrational energy was found to have a substantially lower contribution than normal translation and surface phonons in providing exchangeable energy to react, as shown in Fig. 3.19(a). The mean exchangeable energy of all initial species at $873 \mathrm{~K}$, $\left\langle E_{v}^{e x}\right\rangle=\eta_{v}\left\langle E_{v}\right\rangle$, has an effective temperature of approximately $700 \mathrm{~K}$, and the mean exchangeable energy in vibration for PCs which successfully react would be similarly effected 
$\left\langle E_{v}^{e x}\right\rangle_{R}=\eta_{v}\left\langle E_{v}\right\rangle_{R}$. Interestingly, normal detailed balance arguments seem to become problematic with the dynamical constraint applied to exchangeable energy in vibrational degrees of freedom.

d-PMMT thermal dissociative sticking coefficeints for ambient methane isotopomers are shown in Figure 3.20(a) with the kinetic isotope effect in 3.20(b). Wei and Iglesia ${ }^{6}$ experimentally measured the dissociation of $\mathrm{CH}_{4}$ on $1.6 \mathrm{wt} \% \mathrm{Pt} / \mathrm{ZrO}_{2}$ at $873 \mathrm{~K}$ and determined the kinetic isotope effect to be 1.58 at $873 \mathrm{~K}$, somewhat smaller than the d-PMMT prediction of 2.4. At low temperatures the KIE becomes sufficiently large that tunneling is expected to play a primary role in reaction dynamics.

Figure 3.21(a) shows the thermal dissociative sticking coefficient, $S(T)$, for ambient $\mathrm{CH}_{4}$ gas above an isothermal $\mathrm{Pt}(111)$ surface calculated by the d-PMMT model. Shown for comparison is a direct experimental measurement of $S(T)$ from angular dissociative sticking measurements by $\mathrm{Navin}^{56}$ at $\mathrm{T}=700 \mathrm{~K}$, with an ARD of 30\%. To assess the impact of dynamics on thermal sticking a statistical (s-) PMMT calculation in which all the efficacies of the d-PMMT model were set to their limiting statistical values of one (i.e., $\eta_{i} \rightarrow 1$ ) was performed. At temperatures of catalytic interest, dynamical effects are found to account for a change in sticking by a factor of 7 as compared to the expectation under statistical conditions. From Fig. 3.21(a), the importance of tunneling on thermal sticking from 125 to $1000 \mathrm{~K}$ was determined and is shown in Figure 3.21(b). At catalytically relevant temperatures, tunneling was found to account for a negligible fraction of reactivity, however by $200 \mathrm{~K}$ the fraction of reactivity proceeding via tunneling pathways has become nearly $100 \%$.

\subsection{G. Comparison to Other Work}




\subsection{G.1. Quantum/Classical Dynamics}

The most sophisticated dynamical model applied to the $\mathrm{CH}_{4} / \mathrm{Pt}(111)$ reactive system has been a reduced dimensionality, 5-dimensional quantum mechanical (5D-QM) model which treated $\mathrm{CH}_{4}$ as a pseudo-diatomic molecule and incorporated the motion of one surface atom moving along the direction of the surface normal. ${ }^{22}$ A 5D-QM simulation of dissociative sticking coefficients representative of some of Beck's thermal nozzle supersonic molecular beam experiments (i.e., scanning $\left\langle E_{n}\right\rangle$ at $T_{s}=600 \mathrm{~K}$ ) is shown in Fig. 3.22 and is contrasted with a dPMMT simulation. Although the 5D-QM model study did not attempt to simulate any other $\mathrm{CH}_{4} / \mathrm{Pt}(111)$ experiments, the study concluded that thermal motion of the surface lattice atoms strongly modifies the gas-surface reactivity and that lattice atoms with the largest vibrational energies are the most reactive. Although in agreement that the surface vibrational motion can strongly modulate the gas-surface reactivity, the explicitly 12-D d-PMMT points out that the relative importance of the lattice atoms with the largest vibrational energies falls as the total exchangeable energy of the PCs formed in the beam experiments increases. Generic behavior is observable in the Fig. 3.15 analysis of Beck's supersonic molecular beam experiments that shows $\left\langle E_{s}\right\rangle_{R}$ falls as $\left\langle E_{n}\right\rangle+\eta_{v}\left\langle E_{v}\right\rangle$ increases until a limiting value of $\left\langle E_{s}\right\rangle_{R} \rightarrow\left\langle E_{s}\right\rangle$ is reached. For sufficiently high incident molecular energies the dissociative sticking coefficient does not spatially discriminate between thermal surface lattice atoms with relatively low or high vibrational energies (e.g, no discernment in the limit $S \rightarrow 1$ ). Conversely, if incident molecular energies are very low compared to $E_{0}$, only surface lattice atoms with relatively high vibrational energies can be reactive sites because only these sites can allow formation of PCs with the $E^{*} \geq E_{0}$ required to react. 
The finding that rotation is an apparent spectator to the $\mathrm{CH}_{4} / \mathrm{Pt}(111)$ dissociative chemisorption dynamics through the kinetic modeling of the supersonic and effusive molecular beam experiments presented here is in fairly good accord with expectations based on Jackson's GGA-DFT calculations of the transition state properties. ${ }^{39}$ The GGA-DFT reactive potential energy surface was found to be relatively flat with respect to parallel translation across the unit cell and with respect to molecular rotations near the transition state. Accordingly, the frustrated rotational and parallel translational mode frequencies at the transition state are all relatively low and close to $125 \mathrm{~cm}^{-1}$ except for one rotational motion at $315 \mathrm{~cm}^{-1}$ (see electronic supplemental information for a table of the GGA-DFT transition state properties). Other benchmark systems for gas-surface reaction dynamics for which both parallel translation and rotations are well approximated as spectator degrees of freedom, and quantum state-resolved experimental information is available, include the dissociative chemisorption/recombinative desorption of $\mathrm{H}_{2} / \mathrm{Cu}(111)$ [at least for $\left.E_{r} \leq 40 \mathrm{~kJ} / \mathrm{mol}\right]^{12,65}$ and $\mathrm{CO}_{2}$ dissociative chemisorption/CO oxidation on $\operatorname{Rh}(111){ }^{13}$

The thermally state-averaged vibrational efficacy parameter of $\eta_{v}=0.40$ determined by $\mathrm{d}$ PMMT analysis of the Luntz $T_{N}=680 \mathrm{~K}$ and $300 \mathrm{~K}$ supersonic molecular beam data of Fig. 3.3 is in good quantitative accord with Beck's supersonic molecular beam data and the quantum state-resolved experimental finding of $\eta_{2 v_{3}}=0.38$. These vibrational efficacies are consistent with an early barrier on the reactive potential energy surface according to the Polanyi rules ${ }^{42}$ and, indeed, Jackson's GGA-DFT calculations ${ }^{39}$ indicate that the $\mathrm{CH}_{4} / \mathrm{Pt}(111)$ transition state is early in comparison to $\mathrm{CH}_{4} / \mathrm{Ni}(100)$.

\subsection{G.2. Empirical Erf Model with Distributions of Dynamical Barriers}


A currently popular empirical model of activated gas-surface reactivity posits that as incident molecules in a particular quantum state impact a surface they sample a Gaussian distribution of "dynamical barriers" to reaction that will vary with the molecular quantum state. ${ }^{18,}$ 21, 64 If the normal translational energy of the incident molecule exceeds the local dynamical barrier then reaction occurs with unit efficiency such that the state-resolved dissociative sticking coefficient varies as an error-function (erf) of $E_{n}$ - an "S"-shaped curve on a linear plot. The erf model employs 3 parameters per quantum state at a given surface temperature to fit state-resolved $S\left(\left\langle E_{n}\right\rangle ; E_{v}, E_{r}, T_{s}, \vartheta, \phi\right)$ data of the kind measured by Beck in Fig. 3.4. Rigorous theoretical connection between the erf parameters and features of the reactive potential energy surface has not been demonstrated. Determining erf parameters for a particular quantum state does not provide an ability to predict parameters for other quantum states, nor an ability to predict the outcomes of other experiments. However, the erf formalism has proven useful in evaluating vibrational efficacies from experiments. ${ }^{17,18}$ For polyatomic molecules with many quantum states, the erf model is impractical for predicting thermal dissociative sticking coefficients because too many erf parameters must be determined. Alternatively, the 3-parameter d-PMMT model fit to only the Luntz thermal nozzle data of Fig. 3.3(b) predicts the remaining experimental data of Figs. 3.3, 3.4, 3.5, and 3.10 admirably well and can provide predictions for any conceivable $\mathrm{CH}_{4} / \mathrm{Pt}(111)$ experiment for which $f\left(E^{*}\right)$ in Eq. (3.11) can be defined.

Within the transition state theory framework of the d-PMMT, the "erf-like" state-resolved $S\left(\left\langle E_{n}\right\rangle ; E_{v}, E_{r}, T_{s}, \vartheta, \phi\right)$ curves result from the Eq.(3.3) averaging of the PC active energy distribution $f\left(E^{*}\right)$ over the microcanonical sticking coefficient $S\left(E^{*}\right)$. As illustrated in Fig. 3.2, the breadth of the PC energy distribution, $f_{2 v 3}\left(E^{*}\right)$, in Beck's $2 v_{3}$ state-resolved experiments and the breadth of the thermal equilibrium PC distribution, $f_{T}\left(E^{*}\right)$ are similar and 
both are dominated by the surface phonon distribution. If the incident molecular energy is insufficient to surmount the threshold energy for dissociative chemisorption then it is the tail of the surface phonon distribution that can provide the remaining energy required to assemble some PCs with the $E^{*} \geq E_{0}$ necessary for reaction. The energy accounting of where the energy to react came from for the Beck experiments of Fig. 3.4 is given in Fig. 3.15. At the lowest incident molecular energies only PCs formed at the most thermally excited surface atoms can react, but as the incident molecular energy increases this preferential reactivity at the "hotter" surface sites diminishes.

The semantics of an erf model analysis of Beck's $2 v_{3}$ state-resolved $\mathrm{CH}_{4} / \mathrm{Pt}(111)$ experiments in Fig. 3.4 are unusual. The reactivity at $\left\langle E_{n}\right\rangle=10 \mathrm{~kJ} / \mathrm{mol}$ would seem to indicate that the Gaussian distribution of dynamical barriers extends down to at least $10 \mathrm{~kJ} / \mathrm{mol}$. On single crystal surfaces where step and kink sites reactivity can be experimentally minimized, recognized, or avoided (by poisoning ${ }^{70}$ ), a wide range of reactivity barriers at terrace sites is unexpected. Indeed, electronic structure theory calculations have never found any configuration of Pt atoms and $\mathrm{CH}_{4}$ on a Pt surface that could yield such low barriers on a reactive potential energy surface, including configurations where a Pt atom is pulled well out of the surface plane. ${ }^{39}$ Consequently, the "distribution of dynamical barriers" of the erf model cannot simply arise from the sampling of local barriers on a potential energy surface due to averaging over surface impact site, molecular orientation, and vibrational phase, but rather there must be an admixing of the effects of the availability of surface energy at the site of molecular impact on the surface. Erf parameters are typically found to be $T_{s}$ dependent $^{27,71}$ whereas the potential energy surface is $T_{s}$ independent. The " $E_{a}\left(T_{s}\right) "$ experiments and Tolman analysis above have highlighted the importance of the surface as a flexible energy reservoir that can promote reactivity. It is unfortunate that the 
"distribution of dynamical barriers" has so popularly entered the lexicon of the gas-surface dynamics community because it is often a confounding idea that mixes a description of the potential energy surface and experimental particulars in an ill-defined way, albeit in a way that permits rationalization of the empirical erf model. In the authors view, the d-PMMT model provides a simpler, transition state theory accounting of how "erf-like" state-resolved dissociative sticking coefficients come about and how they relate to the properties of the transition state on the potential energy surface and the experimental particulars.

\subsection{G.3. Electronic Structure Theory}

The $\mathrm{CH}_{4} / \mathrm{Pt}(111)$ dissociative sticking coefficients scale exponentially with the apparent threshold energy for dissociative chemisorption, $E_{0}$. Different electronic structure theory calculations and different kinetic treatments of experimental data have given rise to a range of $E_{0}$ values extending from $43 \mathrm{~kJ} / \mathrm{mol}$ to $121 \mathrm{~kJ} / \mathrm{mol}$ as catalogued in Fig. 3.23(a). The highest $E_{0}$ stems from Madix's analysis of supersonic molecular beam experiments ${ }^{38}$ using a model invoking tunneling through the activation barrier for $\mathrm{C}-\mathrm{H}$ bond breaking at the surface. The earlier MURT (s-PMMT assuming active rotation) analyses of supersonic molecular beam experiments gave $E_{0}$ $=61.3 \mathrm{~kJ} / \mathrm{mol},{ }^{36} 58.9 \mathrm{~kJ} / \mathrm{mol},{ }^{9}$ and lower values of $E_{0}=49 \mathrm{~kJ} / \mathrm{mol},{ }^{66} 48.2 \mathrm{~kJ} / \mathrm{mol}^{16}$ for analyses of different effusive molecular beam experiments. The d-PMMT model value of $E_{0}=58 \mathrm{~kJ} / \mathrm{mol}$ falls within the Fig. 3.23(a) range and allows for the quantitative reproduction of dissociative sticking coefficients for a diverse variety of multidimensional non-equilibrium and state-resolved experiments. Importantly, the d-PMMT model assuming rotation as a spectator is the first model able to simultaneously reproduce the results of both supersonic and effusive molecular beam experiments. The diversity of $E_{0}$ values calculated by GGA-DFT techniques as illustrated in Fig. 3.23 and the $\pm 30 \mathrm{~kJ} / \mathrm{mol}$ accuracy typically estimated ${ }^{23}$ for GGA-DFT barrier heights calculated 
with the most popular functionals are reasons why in the d-PMMT model $E_{0}$ is taken as a parameter to be set by simulation to experiments rather than as a constant to be directly calculated by GGA-DFT. Despite the difficulties noted in calculating absolute energies, ${ }^{23}$ GGA-DFT calculations are believed to provide more quantitative information about local relative energies and transition state configurations. Consequently, the d-PMMT model employed Jackson's GGADFT calculated vibrational frequencies for the $\mathrm{CH}_{4} / \mathrm{Pt}(111)$ transition state ${ }^{39}$ but did not use his zero-point corrected $E_{0}(\mathrm{GGA}-\mathrm{DFT})=79.3 \mathrm{~kJ} / \mathrm{mol}$ value. If the d-PMMT model is run with this

GGA-DFT calculated value of $E_{0}$, i.e., with $\left\{E_{0}=79.3 \mathrm{~kJ} / \mathrm{mol}, s=2, \eta_{v}=0.40\right\}$, the total ARD for the experiments considered in Fig. 3.7 swells to $19,174 \%$ as compared to $43 \%$ with the optimal value of $E_{0}=58 \mathrm{~kJ} / \mathrm{mol}$. Figure 3.23(b) further expounds on the importance and effect of barrier height on reactivity. The difference between reactivity taking the highest and lowest apparent threshold energies spans 5 orders of magnitude at $873 \mathrm{~K}$ and over 12 orders of magnitude at room temperature.

\subsection{G.4. Catalysis}

Recent experimental studies ${ }^{2,6,72,73}$ on dry and steam reforming of methane over a number of metal nanocatalysts at temperatures near $800 \mathrm{~K}$ have established that these processes are structure sensitive and rate limited by methane dissociative chemisorption. Reforming rates were found to scale with the number of metal step or corner sites rather than the terrace sites of the nanocatalyst surfaces. Wei and Igelsia's turnover $\operatorname{rates}^{6}$ for $2 \mathrm{~nm}$ Pt nanocatalysts yield an apparent $\mathrm{CH}_{4}$ thermal dissociative sticking coefficient, $S(T)=S_{0} \exp \left(-E_{a} / R T\right)$, with $S_{0}=3.1$ $\times 10^{-2}$ and $E_{a}=80 \mathrm{~kJ} / \mathrm{mol}^{3}$ The d-PMMT prediction for $S(T)$ on $\operatorname{Pt}(111)$ at similar temperatures is characterized by $S_{0}=0.62$ and $E_{a}=58 \mathrm{~kJ} / \mathrm{mol}$. The near unity pre-exponential 
factor for the $\mathrm{CH}_{4} / \operatorname{Pt}(111) S(T)$ is consistent with reaction at regular terrace sites and a mobile transition state that is not particularly entropically constrained. The smaller pre-exponential factor for the nanocatalyst $S(T)$ might then be construed to result from reaction at minority step sites that may be less sensitive to poisoning by $\mathrm{C}$ build-up, or graphene formation, ${ }^{74}$ than terrace sites. The lower activation energy for $\mathrm{CH}_{4}$ dissociative chemisorption on the clean $\mathrm{Pt}(111)$ terrace sites as compared to the nanocatalyst step sites is again suggestive that the surfaces of the operating reforming nanocatalysts may be modified by adsorbed species.

The d-PMMT model calculates $S(700 \mathrm{~K})=7.1 \times 10^{-6}$, a value 300 higher than the apparent $S(700 \mathrm{~K})$ for $\mathrm{CH}_{4} / 2 \mathrm{~nm} \mathrm{Pt}$ nanocatalysts calculated from steam reforming turnover rates under the common assumption that methane dissociative chemisorption is the rate-limiting step. ${ }^{6}$ Conversely, the dr-PMMT and s-PMMT values are 7 and 14 times higher respectively. The reduction of the methane thermal reactivity from its statistical transition state theory value by a factor of 2 because $\eta_{v}=0.4$ and an additional factor of 7 because rotation is a spectator argues for the importance of dynamical knowledge for quantitative engineering of industrial processes such as hydrogen production by methane reforming ${ }^{6}$ or graphene growth ${ }^{75}$ by methane chemical vapor deposition.

\subsection{Summary}

A dynamically biased precursor mediated microcanonical trapping (d-PMMT) model of activated dissociative chemisorption was developed and applied to the dissociative chemisorption of $\mathrm{CH}_{4}$ on $\mathrm{Pt}(111)$. The d-PMMT model consists of building a dynamical biasing constraint into the assembly of the precursor complexes' active exchangeable energy, $E^{*}$, and calculating experimental dissociative sticking coefficients by averaging the completely statistical, microcanonical sticking coefficient, $S\left(E^{*}\right)$, over the energy distributions, $f\left(E^{*}\right)$, of 
the precursor complexes experimentally formed, as in Eq. (3.3). The value of the dynamical biasing constraint, the state-averaged vibrational efficacy $\left(\eta_{v}=0.40\right)$, is believed to relate to the early/late character of the reactive transition state. The dissociative sticking coefficients of effusive and supersonic molecular beam experiments could only be simultaneously reproduced by assuming molecular rotation is a spectator to the dissociative chemisorption dynamics. The determination that rotation is a spectator decreases predictions of thermal dissociative sticking coefficients extrapolated from supersonic molecular beam experiments by roughly an order of magnitude. Surface phonons, and not the incident molecules, are predicted to play the dominant role in providing the energy required to surmount the activation barrier to $\mathrm{CH}_{4} / \mathrm{Pt}(111)$ dissociative chemisorption under the thermal equilibrium conditions relevant to catalysis. The effects of rotation's spectator status and vibrational efficacy of $\eta_{v}=0.4$ were found to account for a factor of 14 change in methane reactivity at $873 \mathrm{~K}$. At temperatures below ambient, tunneling was found to play an increasingly dominant role in methane reactivity, eventually becoming the sole means of reactivity at $200 \mathrm{~K}$. The d-PMMT model was used to connect and compare the results of electronic structure theory and varied kinds of experiments to one another after fixing its parameters $\left\{E_{0}=57.9 \mathrm{~kJ} / \mathrm{mol}, s=2, \eta_{v}=0.40\right\}$ to the results of a limited subset of supersonic molecular beam experiments from a single laboratory. The finding that the d-PMMT model reproduces a diverse variety of $\mathrm{CH}_{4} / \mathrm{Pt}(111)$ experimental results provides encouragement that the methane dissociative chemisorption can be largely understood within the general framework of transition state theory subject to a modest number of constraints (i.e., spectator rotations \& parallel translations, and a state-averaged vibrational efficacy, $\eta_{v}$ ). 

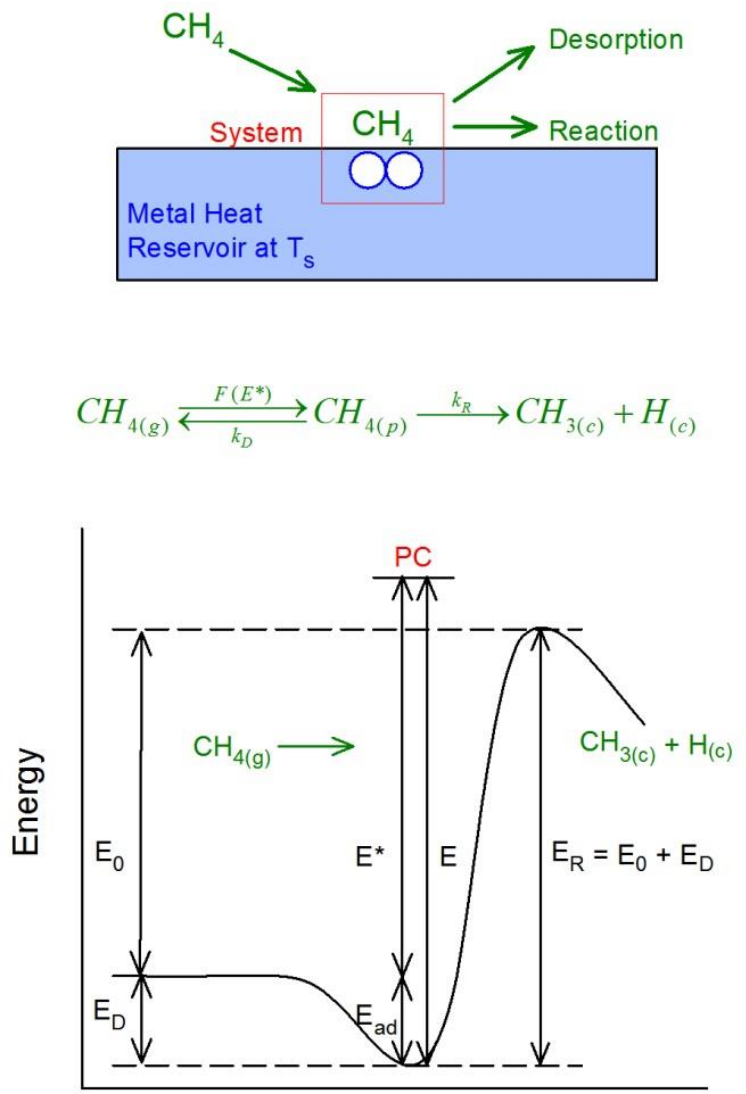

Reaction Coordinate

Figure 3.1. Schematic depiction of the kinetics and energetics of methane dissociative chemisorption. At energies sufficient to access the transition state region of the potential energy surface, microcanonical trapping is presumed to occur for collisionally formed physisorbed complexes, PCs, comprised of a methane molecule interacting with $s$ surface oscillators in the spatial vicinity of the physisorption well minimum. Zero-point energies are implicitly included within the potential energy curve along the reaction coordinate. Refer to the text for further details. 


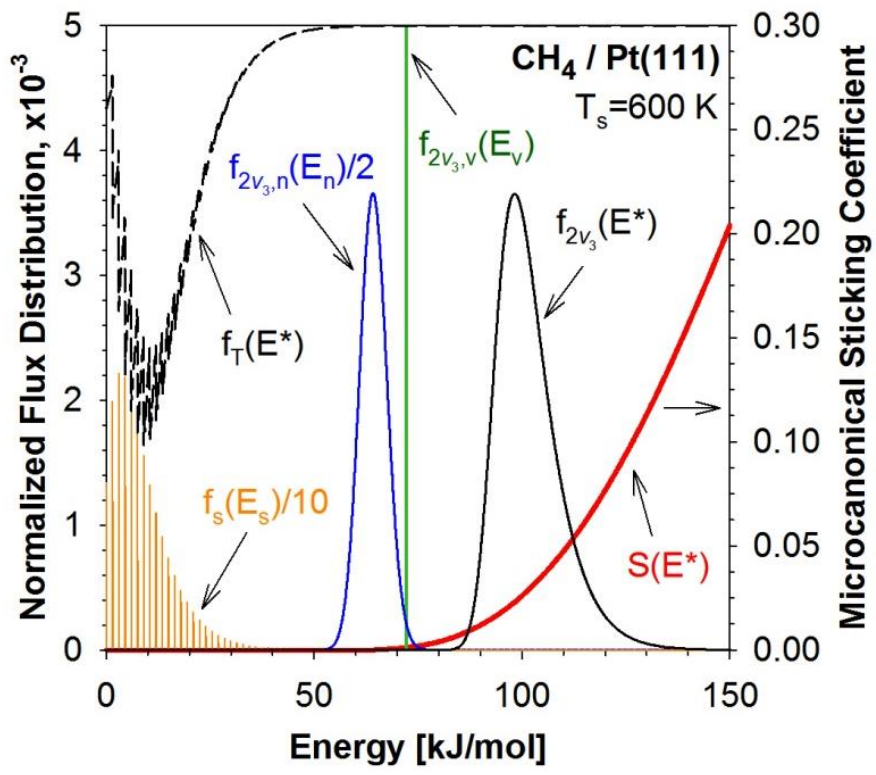

Figure 3.2. Surface, vibrational, and normal translational energy distributions of the $\left\langle E_{n}\right\rangle=64 \mathrm{~kJ} / \mathrm{mol},\left(2 v_{3}, J=2\right)$ state-resolved supersonic molecular beam experiment of Fig. 4(b) are shown along with the convolved active exchangeable energy distribution, $f_{2 v_{3}}\left(E^{*}\right)$, of the PCs formed for the d-PMMT model with rotations and translations parallel to the surface taken as spectators. The PC active energy distribution $f_{T}\left(E^{*}\right)$ appropriate to the thermal equilibrium dissociative sticking for an ambient gas/surface system at $T=T_{g}=T_{s}=600 \mathrm{~K}$ is shown flipped upside down for comparison. Averaging these $f\left(E^{*}\right)$ s sharing the same $T_{s}$ over the microcanonical sticking coefficient, $S\left(E^{*}\right)$, depicted at right yields supersonic beam and thermal equilibrium dissociative sticking coefficients of $6.5 \times 10^{-2}$ and $1.8 \times 10^{-6}$, respectively. The d-PMMT parameters are $\left\{E_{0}=57.9 \mathrm{~kJ} / \mathrm{mol}, s=2, \eta_{v}=0.40\right\}$. 


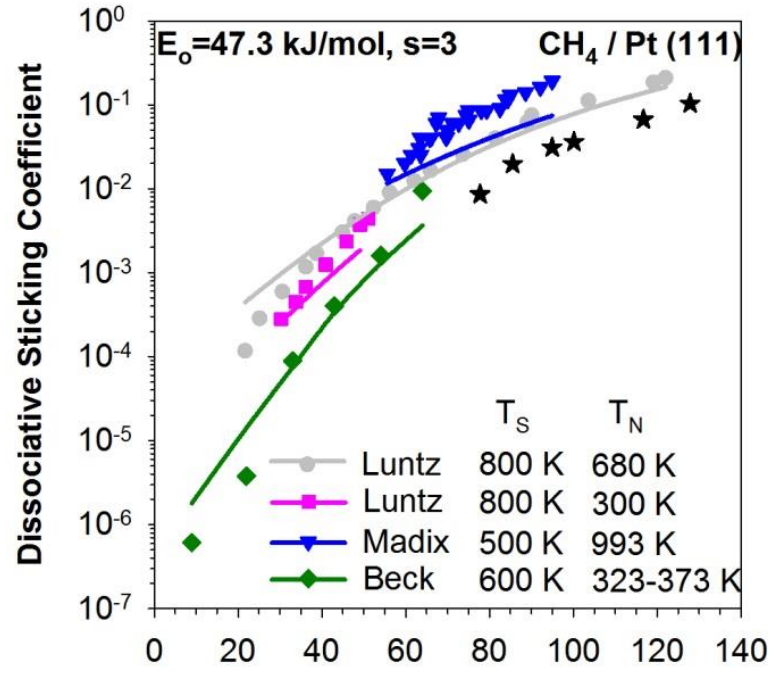

(a) Normal Translational Energy, $\left\langle\mathrm{E}_{\mathrm{n}}>[\mathrm{kJ} / \mathrm{mol}]\right.$

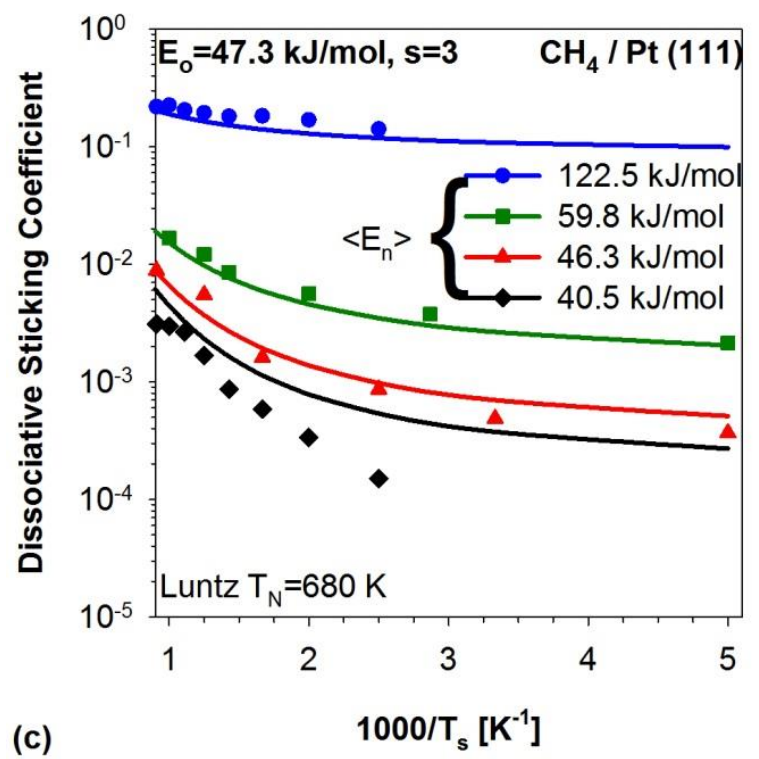

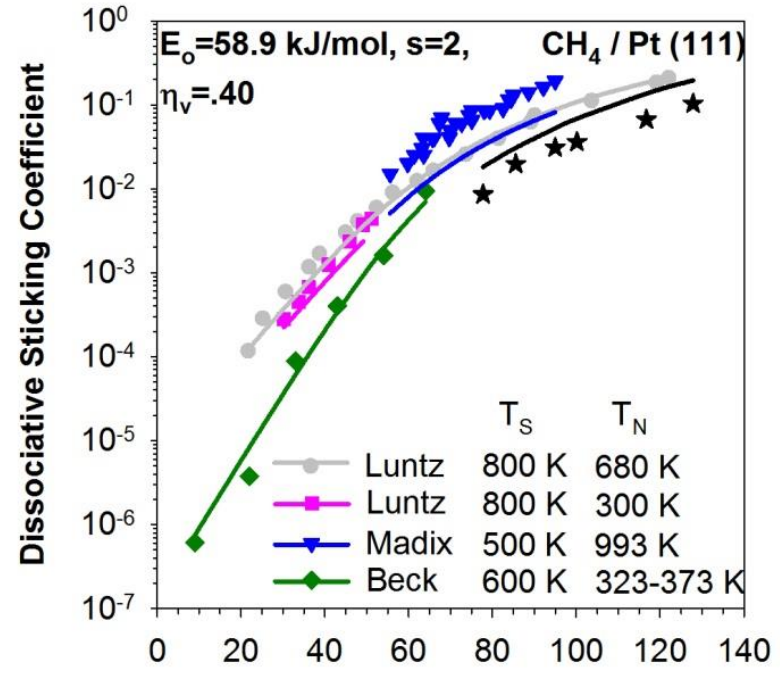

(b) Normal Translational Energy, $<\mathrm{E}_{\mathrm{n}}>[\mathrm{kJ} / \mathrm{mol}]$

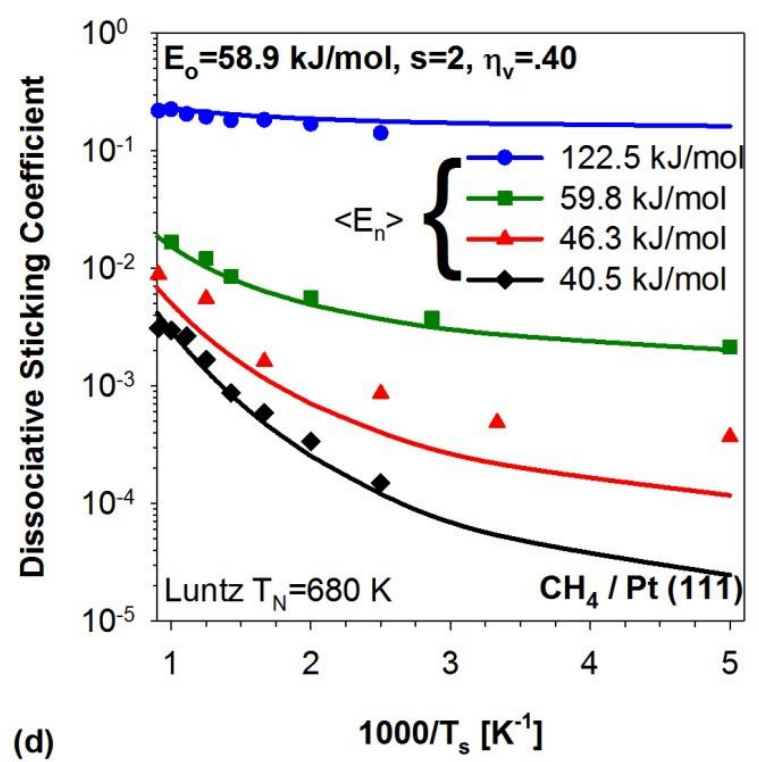

Figure 3.3. Dissociative sticking coefficients for supersonic molecular beams of $\mathrm{CH}_{4}$ incident on Pt(111). Experimental data (points) from the Luntz, ${ }^{30,53} \mathrm{Beck}^{21}$ and Madix ${ }^{38}$ laboratories are compared to two PMMT models (lines) whose parameters were optimized to the Luntz experiments depicted in (a) \& (b). (a),(c) s-PMMT simulations with active rotations and no vibrational biasing. (b),(d) d-PMMT simulations with rotation as a spectator and vibrational efficacy parameter, $\eta_{v}$. Simulations are also provided for the $\mathrm{CD}_{4}$ experiments by Luntz. 


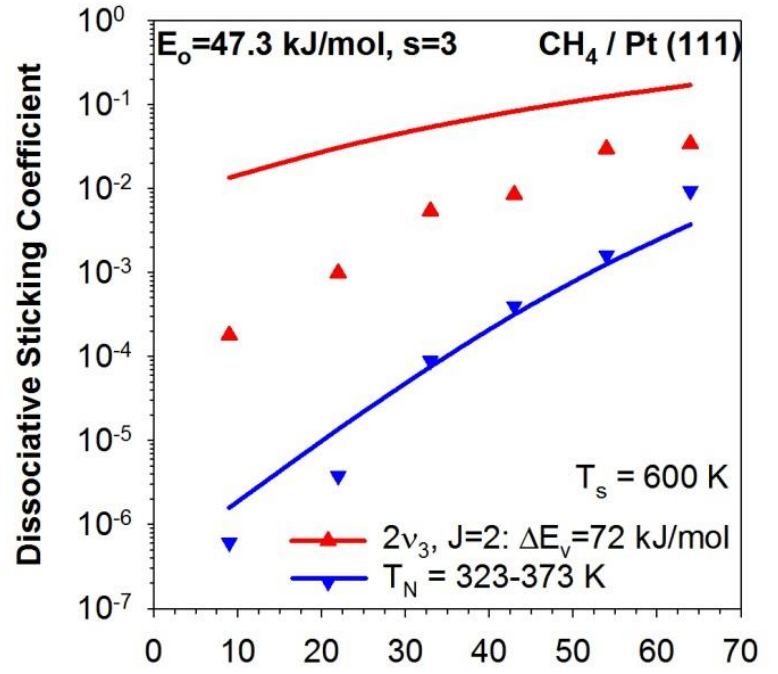

(a) Normal Translational Energy, $<\mathrm{E}_{\mathrm{n}}>[\mathrm{kJ} / \mathrm{mol}]$

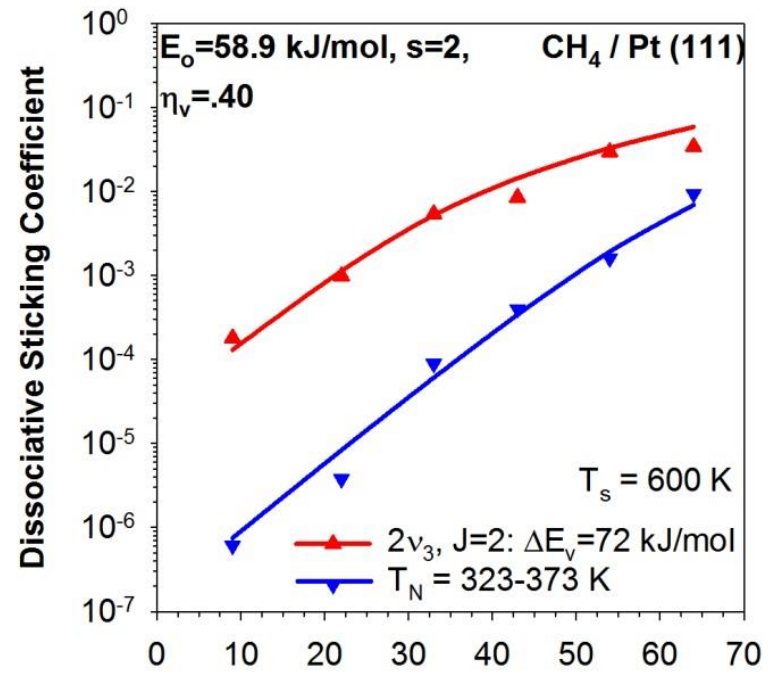

(b) Normal Translational Energy, $<\mathrm{E}_{\mathrm{n}}>[\mathrm{kJ} / \mathrm{mol}]$

Figure 3.4. Dissociative sticking coefficients for a thermal nozzle and a $\left(2 v_{3}, J=2\right)$ state-resolved supersonic molecular beam of $\mathrm{CH}_{4}$ incident on $\mathrm{Pt}(111)$. Beck's experiments ${ }^{21}$ (points) are compared to two theoretical models (lines). 


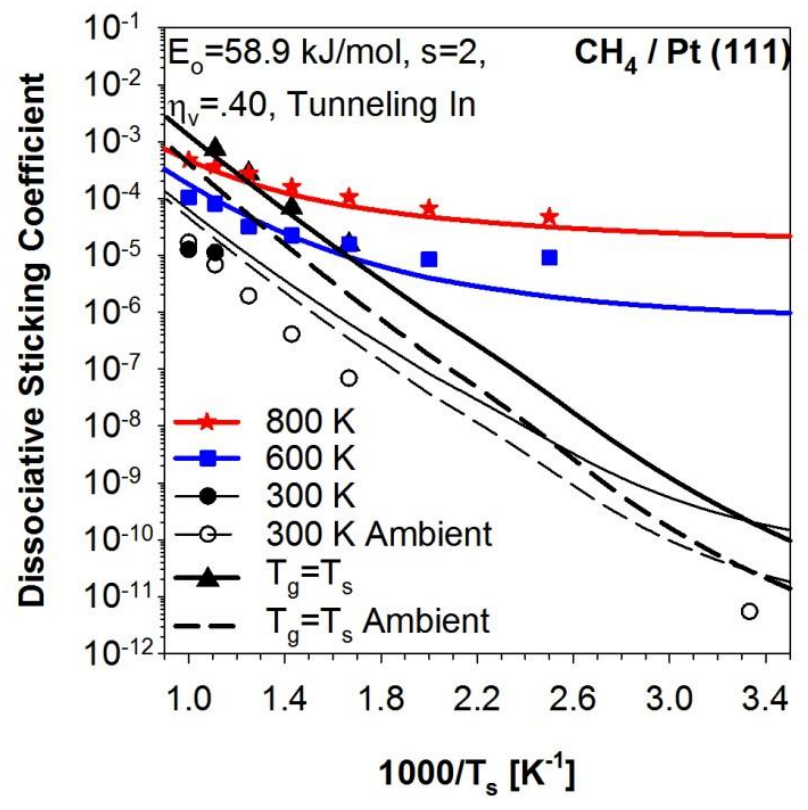

Figure 3.5. Dissociative sticking coefficients for thermal effusive molecular beams of $\mathrm{CH}_{4}$ incident on $\mathrm{Pt}(111)$ along the direction of the surface normal and in one case for a $300 \mathrm{~K}$ ambient gas. The legend gives the impinging gas temperatures, $T_{g}$. Cushing's experiments $^{15,16}$ (points) are compared to d-PMMT predictions (lines). 


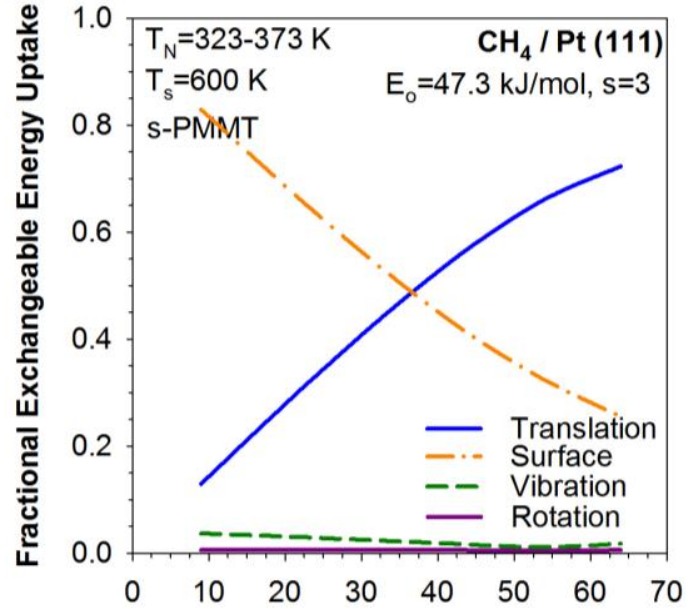

(a)

Normal Translation, $\left\langle\mathrm{E}_{n}\right\rangle[\mathrm{kJ} / \mathrm{mol}]$

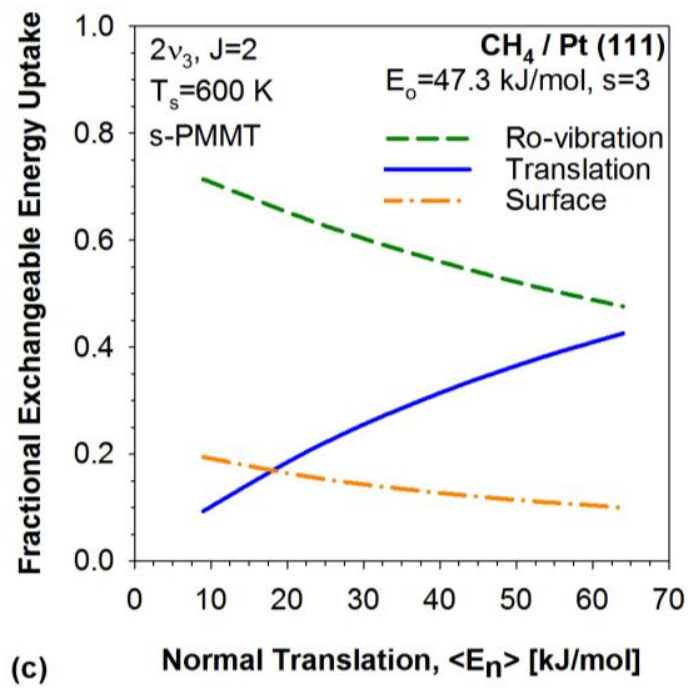

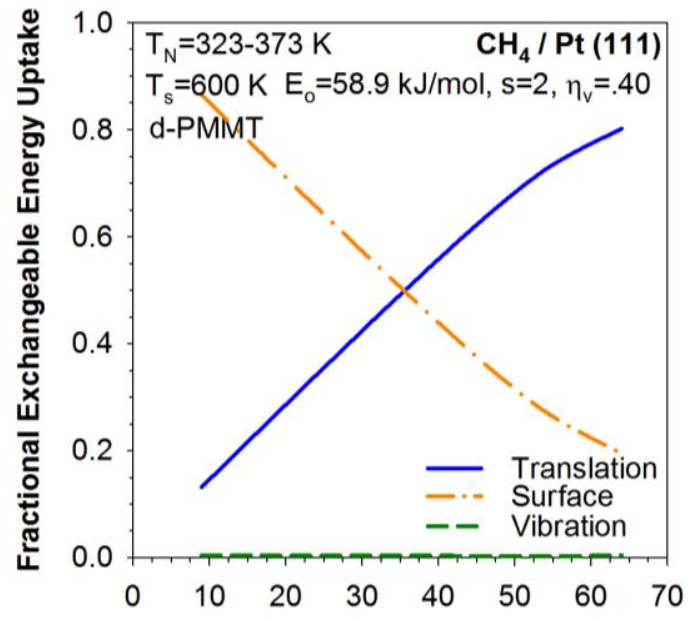

(b)

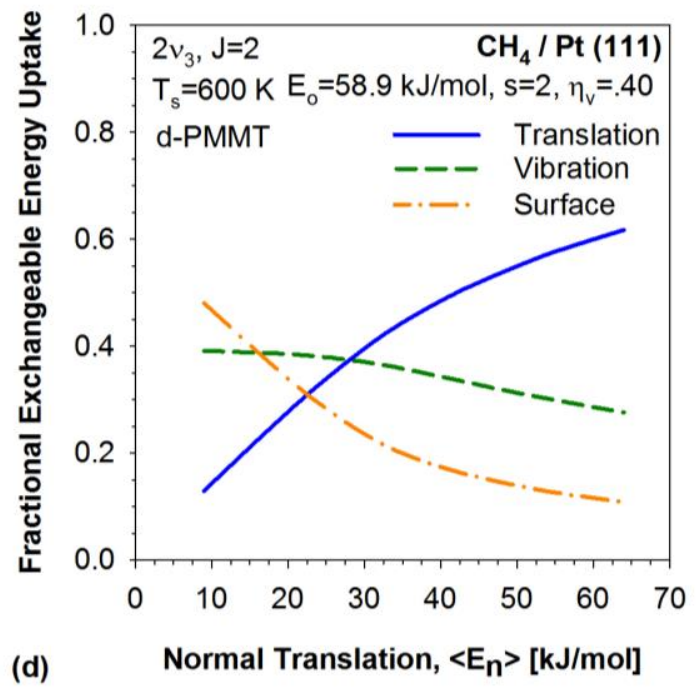

Figure 3.6. Fractional exchangeable energy uptakes for Beck's thermal and $\left(2 v_{3}, J=2\right)$ state-resolved supersonic molecular beam experiments ${ }^{21}$ performed at $T_{s}=600 \mathrm{~K}$ for two different MURT models whose parameters were optimized to the Luntz experiments ${ }^{30,53}$ of Figs. 3(a),(b). (a),(c) s-PMMT simulations with active rotations and no vibrational biasing. (b),(d) d-PMMT simulations with rotation as a spectator and vibrational efficacy parameter, $\eta_{v}$. Compare to Fig. 8. 


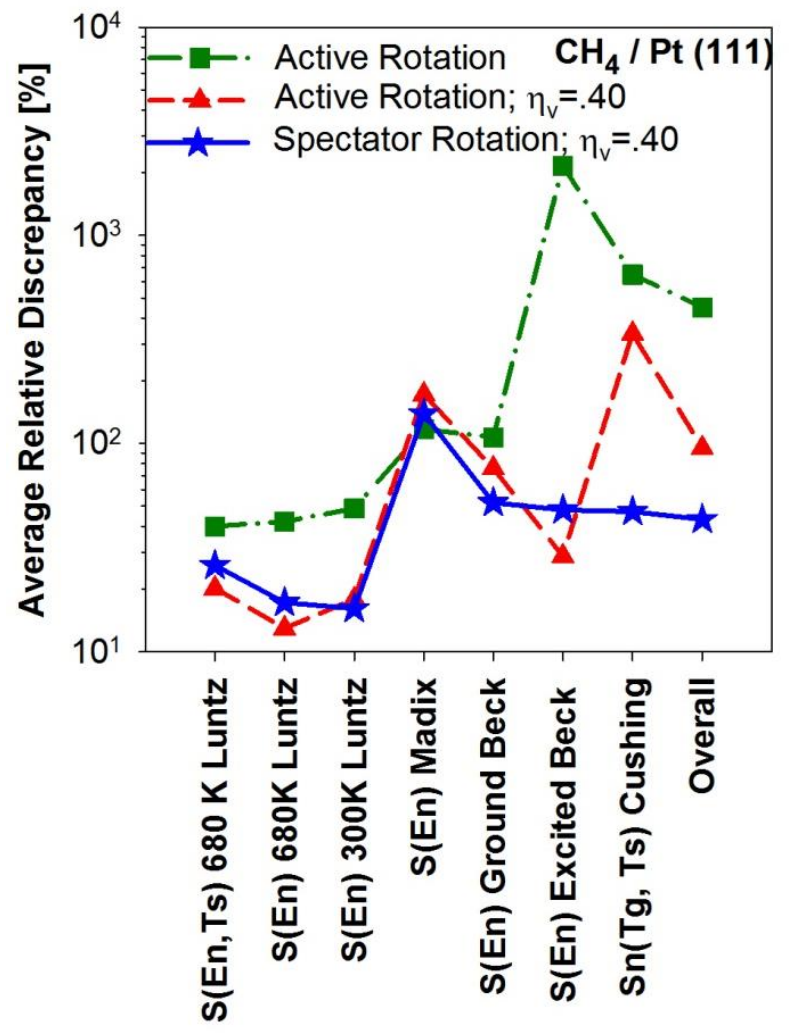

Figure 3.7. The average relative discrepancy, $A R D=\left\langle\left|S_{\text {theory }}-S_{\text {expt }}\right| / \min \left(S_{\text {theory }}, S_{\text {expt }}\right)\right\rangle$, is compared for the different $\mathrm{CH}_{4} / \mathrm{Pt}(111)$ experimental data against three theoretical models. 


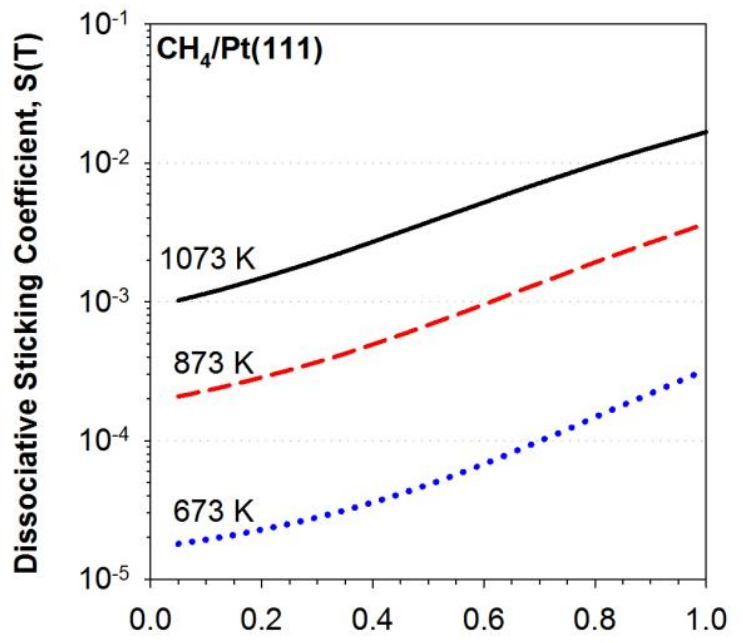

(a)

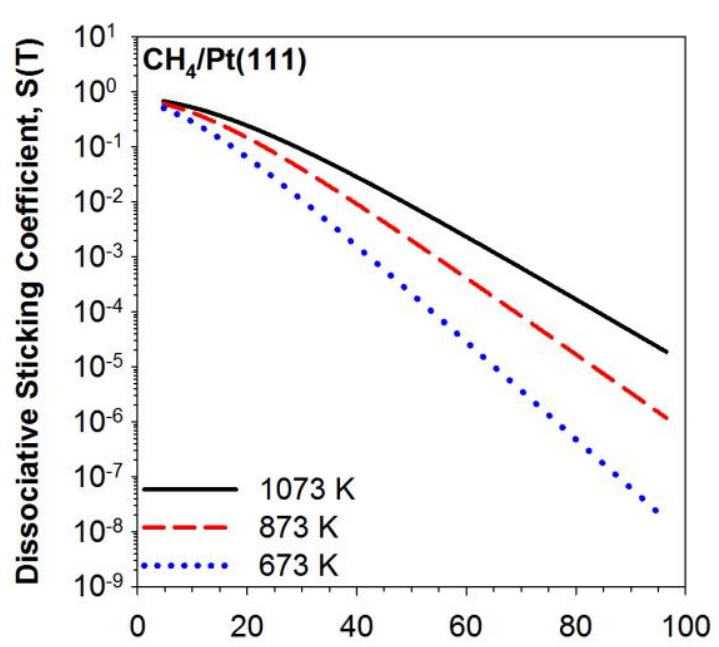

(b)

Threshold Energy, $E_{o}[\mathrm{~kJ} / \mathrm{mol}]$

Figure 3.8. Thermal dissociative sticking coefficients calculated from d-PMMT simulations of the chemisorption of methane on $\mathrm{Pt}(111)$ independently varying only the (a) vibrational efficacies and (b) threshold energy for reaction parameters. 


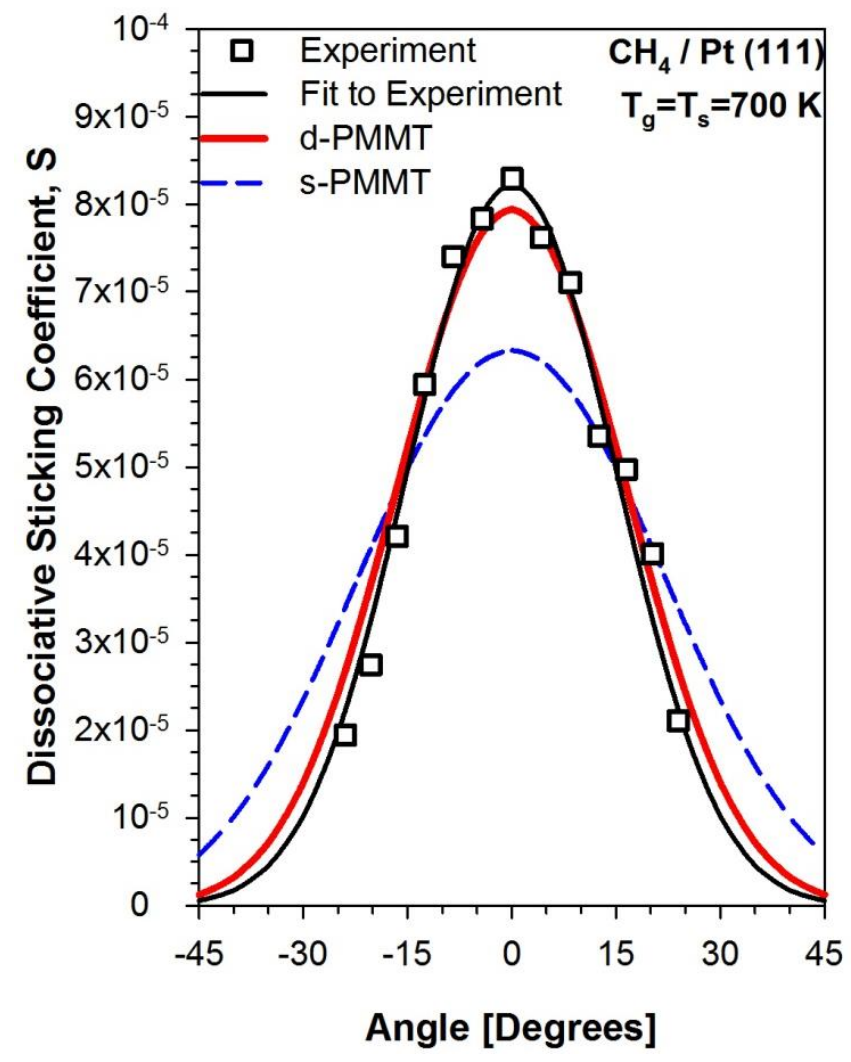

Figure 3.9. Effusive molecular beam measurements of the $\mathrm{CH}_{4} / \mathrm{Pt}(111)$ angle-resolved thermal dissociative sticking coefficient at $700 \mathrm{~K}$ (open points) are compared to angular distribution predictions (lines) from several theoretical models whose parameters were optimized to a limited set of supersonic molecular beam experiments performed at a nozzle temperature of $680 \mathrm{~K}$ and $300 \mathrm{~K}$. The parameters and $S(700 \mathrm{~K}, \vartheta)$ predictions of the models are given in Table 3.1. The $S(700 \mathrm{~K}, \vartheta)$ predictions scaled by the divisors yielding the best fits to the experimental data appear as the lines of this figure. 


\begin{tabular}{|c|c|c|c|c|c|c|c|c|}
\hline Model & $E_{0}(\mathrm{~kJ} / \mathrm{mol})$ & $s$ & $\eta_{v}$ & $\eta_{r}$ & $S(700 \mathrm{~K}, \vartheta)$ & $\begin{array}{l}\text { Unscaled } \\
\text { ARD (\%) }\end{array}$ & $\begin{array}{l}\text { Sticking } \\
\text { Divisor }\end{array}$ & $\begin{array}{l}\text { Scaled } \\
\text { ARD (\%) }\end{array}$ \\
\hline s-PMMT & 47.3 & 3 & 1 & 1 & $4.5 \times 10^{-4} \cos ^{6.9} \vartheta$ & 660 & 7.1 & 26.3 \\
\hline dr-PMMT & 44.4 & 2 & 0.4 & 1 & $2.5 \times 10^{-4} \cos ^{8.8} \vartheta$ & 283 & 3.4 & 20.0 \\
\hline d-PMMT & 58.9 & 2 & 0.4 & 0 & $4.1 \times 10^{-5} \cos ^{12.1} \vartheta$ & 68.9 & 0.57 & 12.1 \\
\hline$S_{0} \cos ^{n} \vartheta$ Fit & \multicolumn{4}{|c|}{ Experiment } & $8.2 \times 10^{-5} \cos ^{14.5} \vartheta$ & 7.9 & 1 & 7.9 \\
\hline
\end{tabular}

Table 3.1. Comparison of experimental $\mathrm{CH}_{4} / \mathrm{Pt}(111)$ angle-resolved thermal dissociative sticking coefficients to d-PMMT calculations with varying exchangeable energies in rotational and vibrational degrees of freedom. 

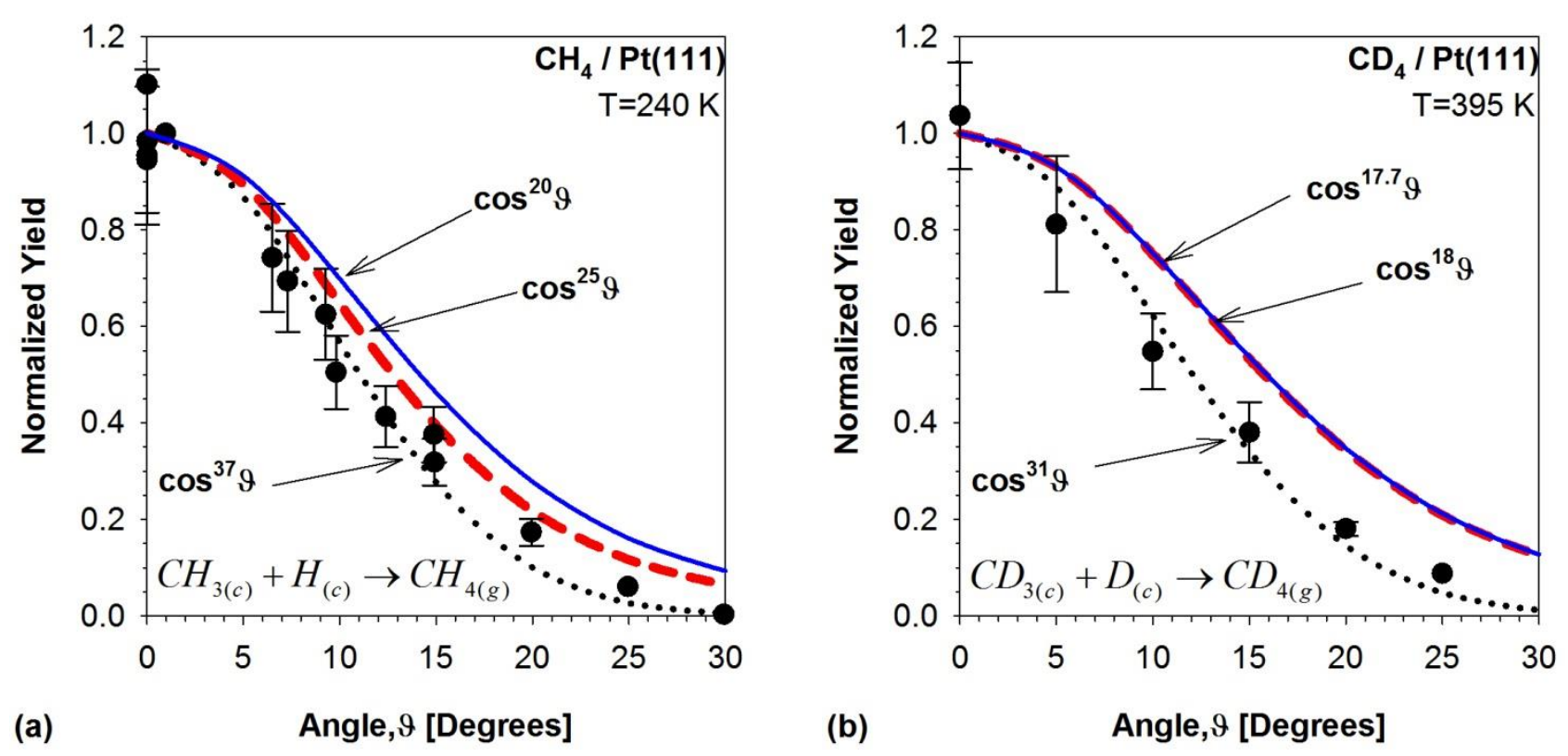

Figure 3.10. Detailed balance d-PMMT simulations with (solid lines) and without (dashed lines) tunneling are compared to experimental data (points with dotted line fits) product angular distributions for (a) thermally induced methyl radical hydrogenation on $\operatorname{Pt}(111)$ on a $240 \mathrm{~K}$ surface and (b) the LITR $C D_{3(c)}+D_{(c)} \rightarrow C D_{4(g)}$ on $\operatorname{Pt}(111)$ assumed to occur at the calculated reaction peak surface temperature of $395 \mathrm{~K}^{76}$. 

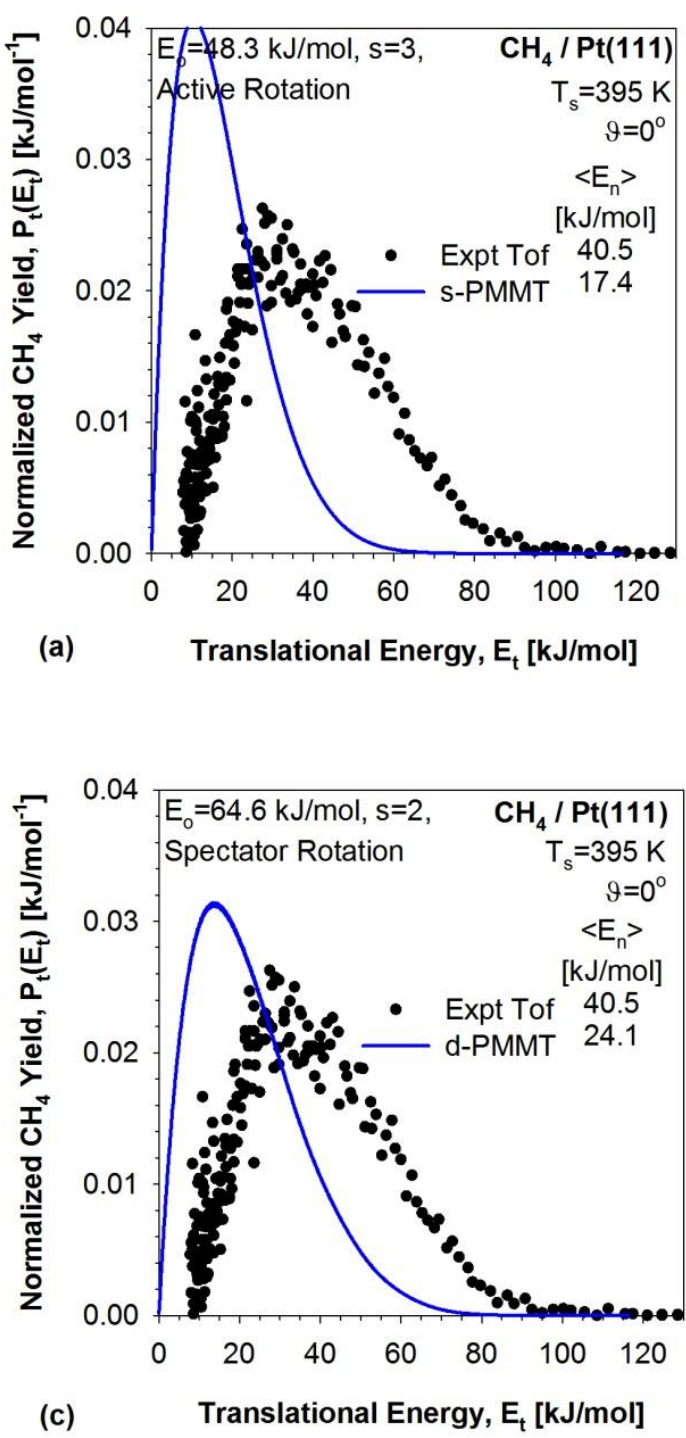

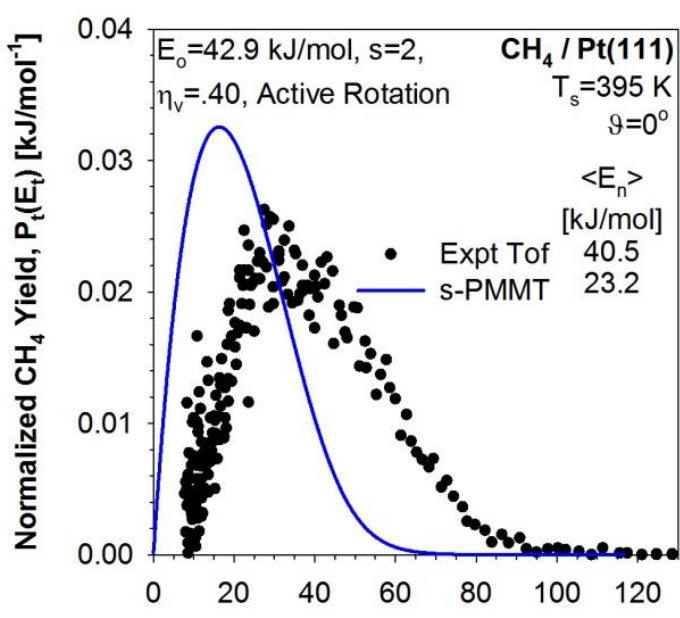

(b)

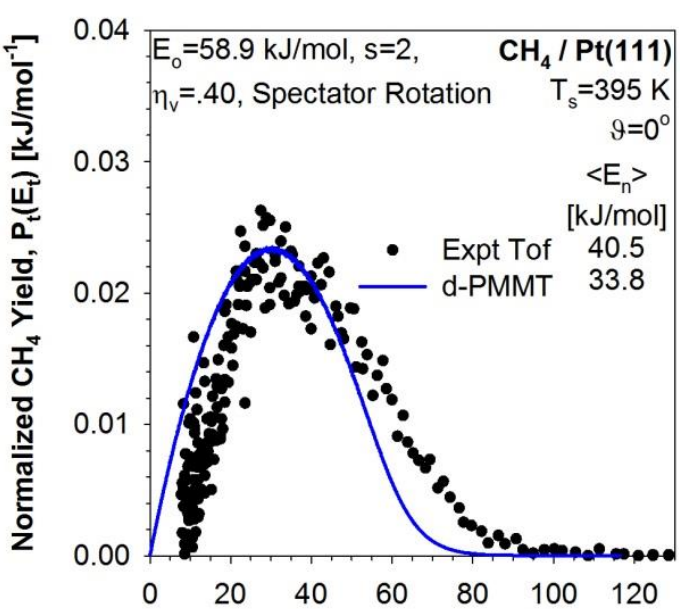

(d)

Translational Energy, $E_{t}[\mathrm{~kJ} / \mathrm{mol}]$

Figure 3.11. Experimental data ${ }^{59,76}$ (points) and detailed balance theoretical simulations (lines) are compared for the $\mathrm{CH}_{4(g)}$ product translational energy distributions derived from time-of-flight spectra of the laser induced thermal reaction $\mathrm{CH}_{3(c)}+\mathrm{H}_{(c)} \rightarrow \mathrm{CH}_{4(g)}$ on $\mathrm{Pt}(111)$. (a) s-PMMT simulations with active rotations and no vibrational biasing. (b) d-PMMT simulations with active rotations and vibrational efficacy parameter, $\eta_{v}$. (c) s-PMMT simulations with rotation as a spectator and no vibrational biasing. (d) d-PMMT simulations with rotation as a spectator and vibrational efficacy parameter, $\eta_{v}$. 


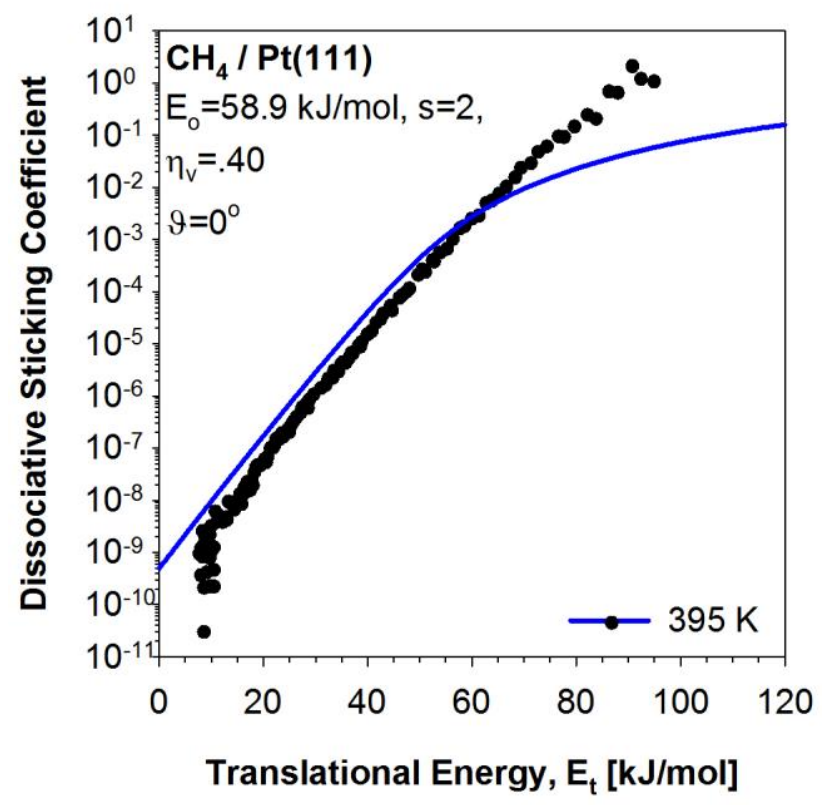

Figure 3.12. Dissociative sticking coefficients, $S\left(E_{t}, \vartheta=0^{\circ} ; T\right)$, (points) derived from the thermal associative desorption product energy distributions of Fig. 3(d) are normalized by and compared to d-PMMT simulations of absolute dissociative sticking coefficients (lines). 


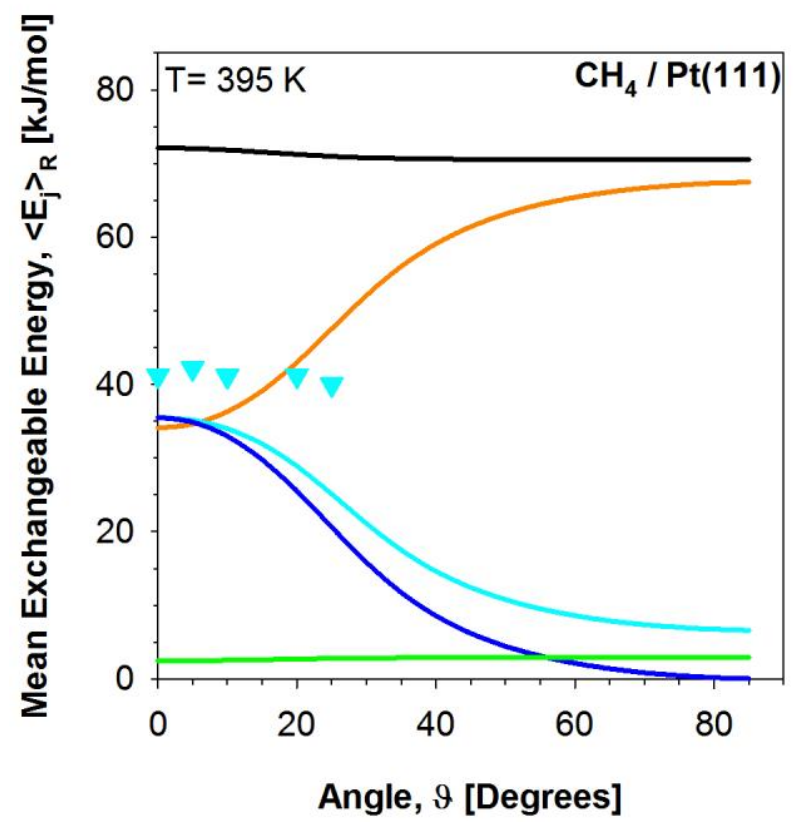

Figure 3.13. Mean energies, $\left\langle E_{j}\right\rangle_{R}$, for the $j^{\text {th }}$ degrees of freedom of the successfully reacting reagents (products) of thermal dissociative chemisorption (associative desorption), $\mathrm{CH}_{4(\mathrm{~g})} \rightarrow \mathrm{CH}_{3(c)}+H_{(c)}$, at $\mathrm{T}=395 \mathrm{~K}$ as a function of angle for d-PMMT simulations are compared to experimental value for $\left\langle E_{t}(\vartheta)\right\rangle$ 


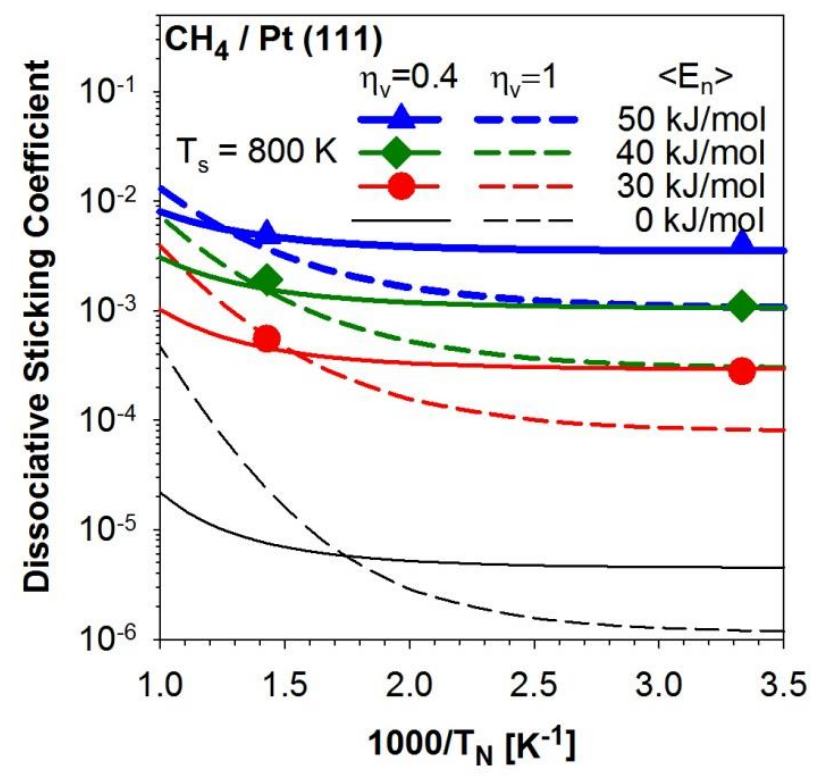

Figure 3.14. Effect of supersonic molecular beam nozzle temperature on $\mathrm{CH}_{4} / \mathrm{Pt}(111)$ dissociative sticking coefficients. Luntz's experiments ${ }^{30,53}$ (points) at several fixed $\left\langle E_{n}\right\rangle$ are compared to the two theoretical models (lines) of Figs 3-5. 


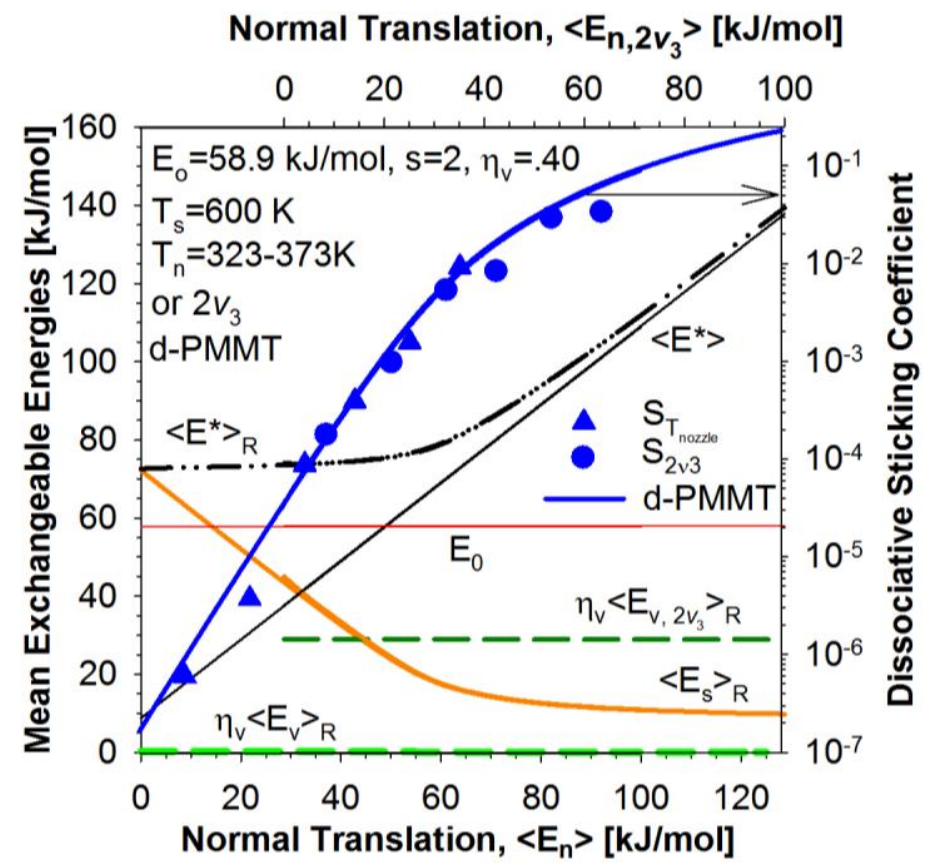

Figure 3.15. Dissociative sticking coefficients and mean energies, $\left\langle E_{j}\right\rangle_{R}$, that derived from the $j^{\text {th }}$ degrees of freedom for the PCs that successfully react as a function of $E_{n}$ for Beck's thermal nozzle and $\left(2 v_{3}, J=2\right)$ state-resolved supersonic molecular beam experiments. $^{21}$ Means of the exchangeable energy, $E^{*}=E_{n}+\eta_{v} E_{v}+E_{s}$, for the successfully reacting PCs, $\left\langle E^{*}\right\rangle_{R}$, and for all the PCs formed, $\left\langle E^{*}\right\rangle$, are also shown. 


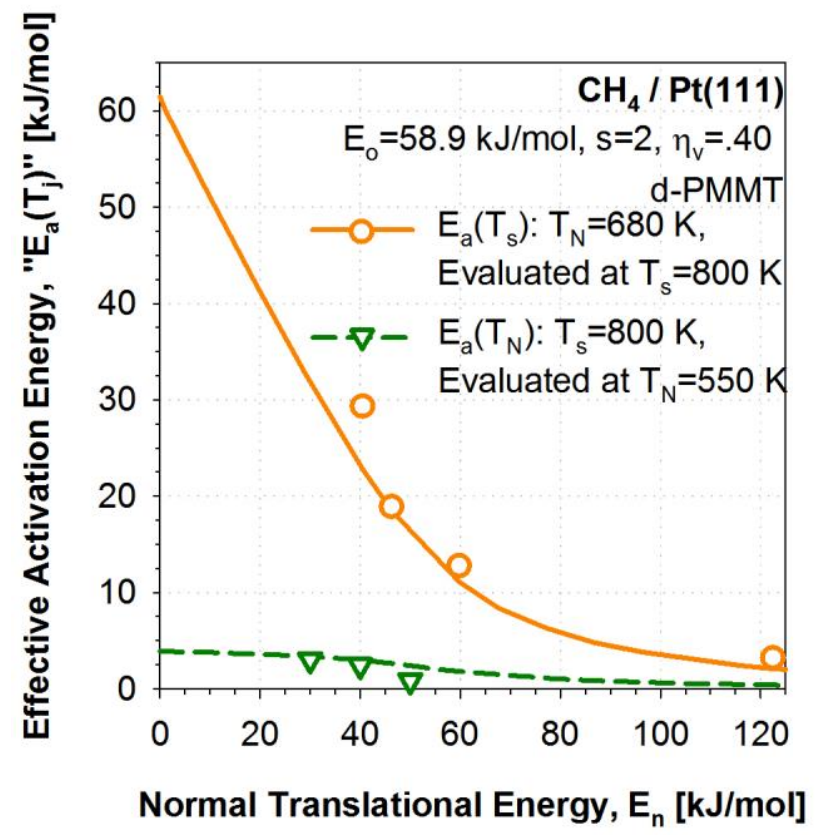

Figure 3.16. Effective activation energies, " $E_{a}\left(T_{j}\right) "=\left\langle E_{j}\right\rangle_{R}-\left\langle E_{j}\right\rangle$, calculated from the Luntz supersonic molecular beam data of Figs. 3(d) and 7 are compared to d-PMMT model predictions. Experimentally based $" E_{a}\left(T_{j}\right) "=k_{B} T_{j}^{2} \partial \ln S\left(T_{j}\right) / \partial T_{j}$ values were calculated based on the $\ln S\left(T_{j}\right)$ data points straddling the evaluation temperatures. 


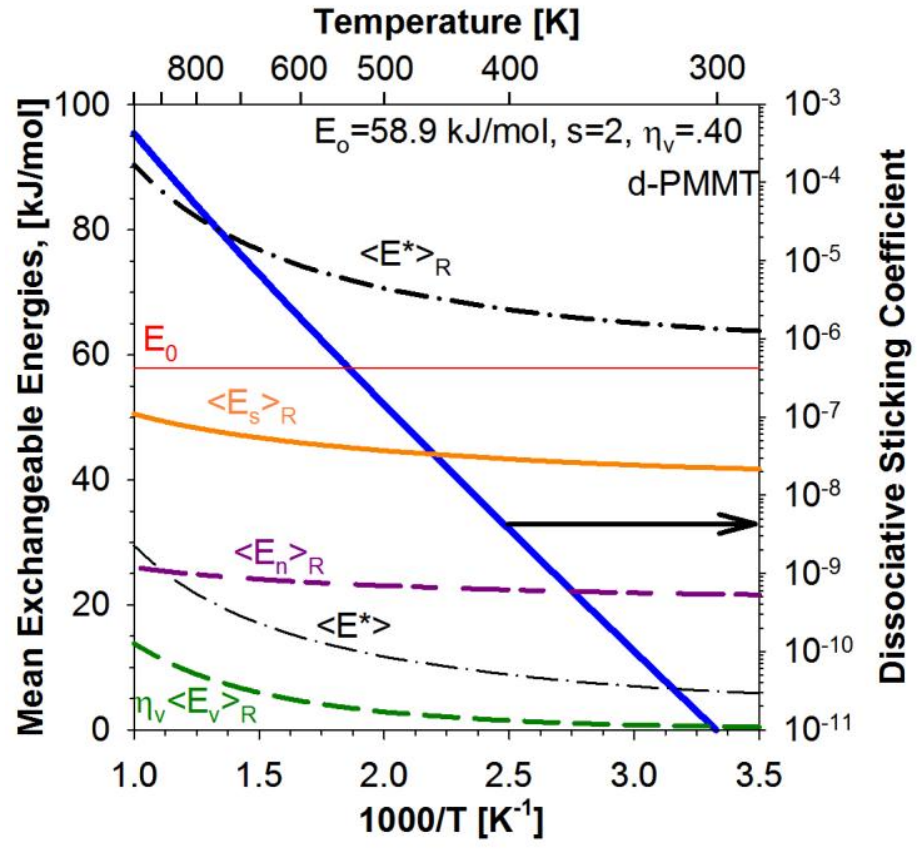

Figure 3.17. Thermal dissociative sticking coefficient and energy accounting for the dissociative chemisorption of $\mathrm{CH}_{4}$ on $\mathrm{Pt}(111)$ under thermal equilibrium conditions. 

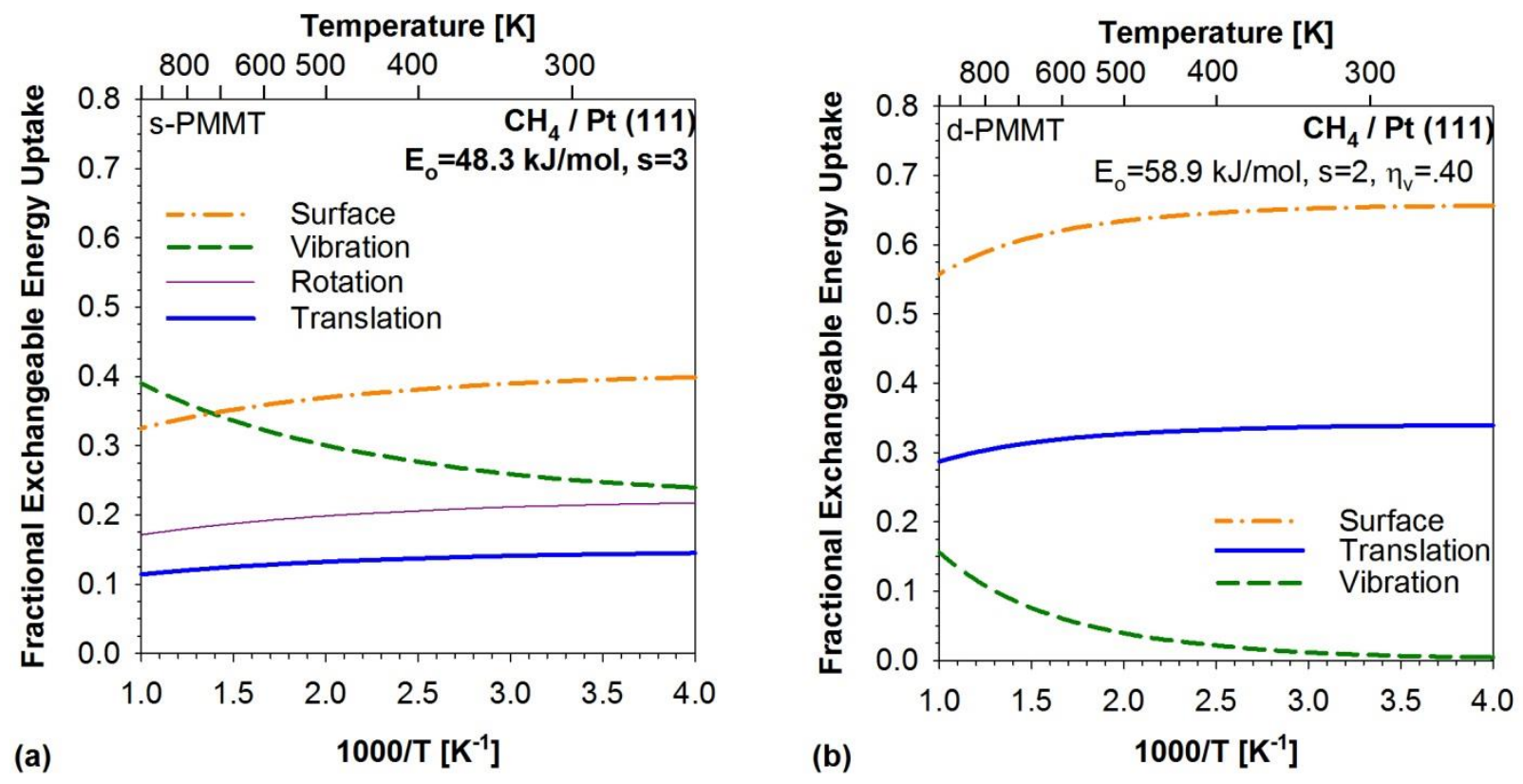

Figure 3.18. Fractional exchangeable energy uptakes, $f_{j}=\left\langle E_{j}\right\rangle_{R} /\left\langle E^{*}\right\rangle_{R}$ for $j \neq v$ and $f_{v}=\eta_{v}\left\langle E_{j}\right\rangle_{R} /\left\langle E^{*}\right\rangle_{R}$, for thermal dissociative chemisorption, where $\left\langle E_{j}\right\rangle_{R}$ and $\left\langle E^{*}\right\rangle_{R}$ are the mean energies derived from the $j^{\text {th }}$ degrees of freedom and the mean exchangeable energy, respectively, for the successfully reacting PCs. 


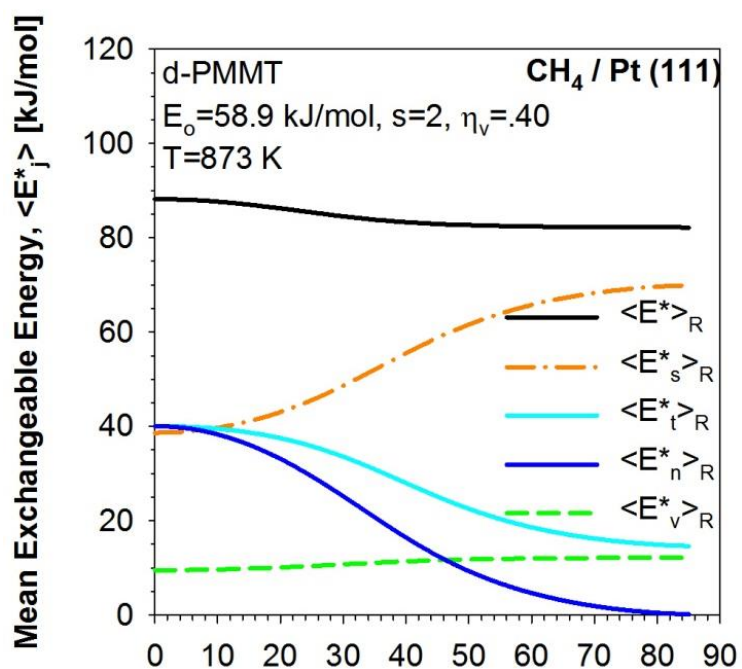

(a)

Angle, 9 [Degrees]

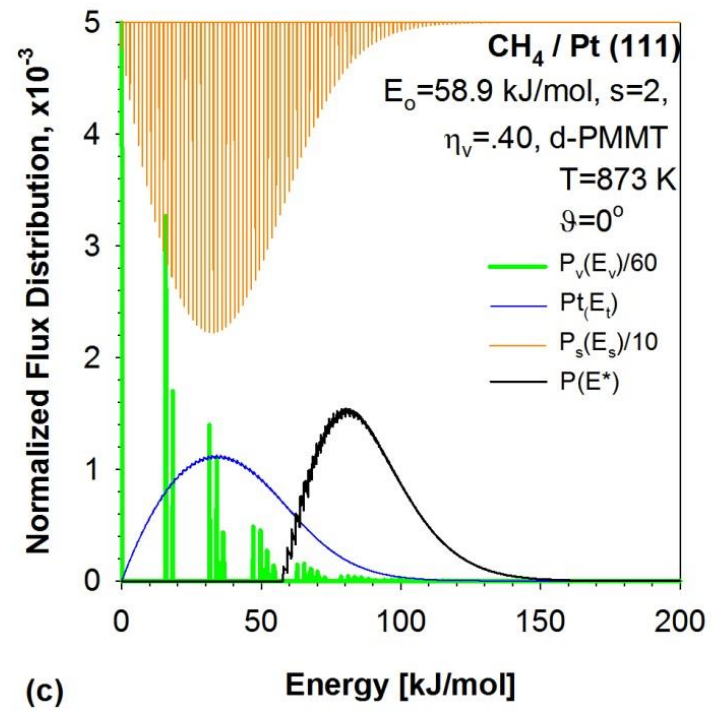

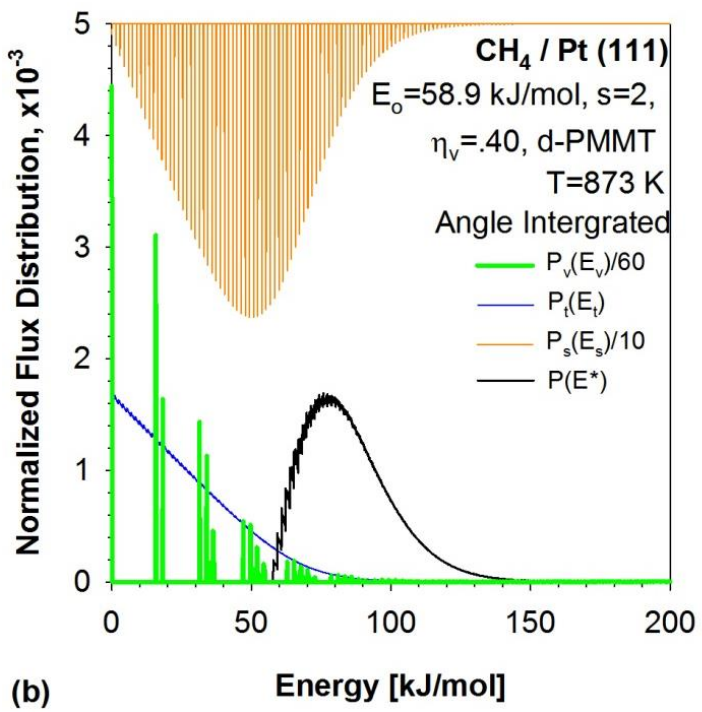

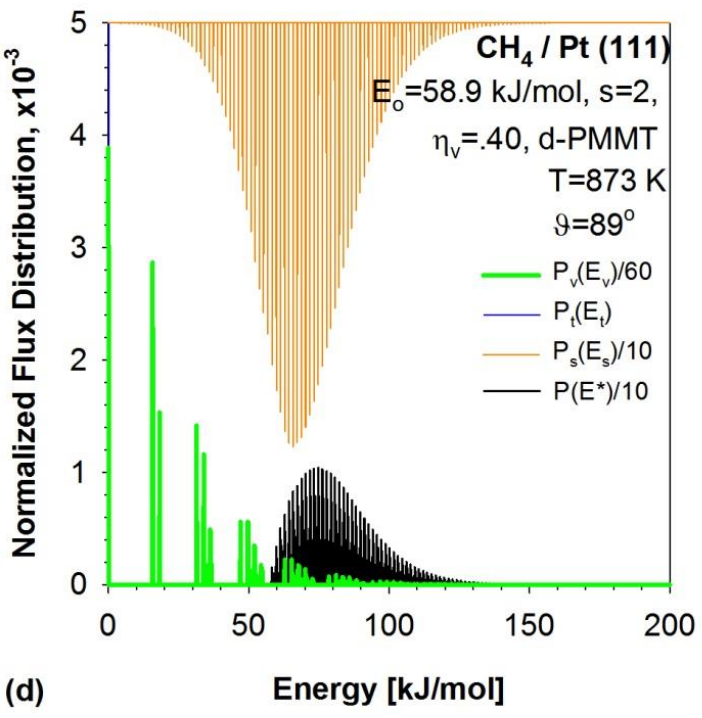

Figure 3.19. (a) Mean energies, $\left\langle E_{j}\right\rangle_{R}$, for the $j^{\text {th }}$ degrees of freedom of the successfully reacting reagents (products) of thermal dissociative chemisorption (associative desorption), $\mathrm{CH}_{4(\mathrm{~g})} \rightarrow \mathrm{CH}_{3(c)}+\mathrm{H}_{(c)}$, at $\mathrm{T}=873 \mathrm{~K}$ as a function of angle d-PMMT simulations. (b) Angle integrated, successfully-reacting-reagents (product) state distributions for thermal dissociative chemisorption (associative desorption) at $\mathrm{T}=873 \mathrm{~K}$ for d-PMMT simulations. (c) Successfully-reacting-reagents (product) state distributions for thermal dissociative chemisorption (associative desorption) for methane incident (desorbing) along the direction of the surface normal at $\mathrm{T}=873 \mathrm{~K}$ for d-PMMT simulations. (d) Successfully-reacting-reagents (product) state distributions for thermal dissociative chemisorption (associative desorption) for methane incident (desorbing) at 89 degrees to the surface normal at $\mathrm{T}=873 \mathrm{~K}$ for $\mathrm{d}-\mathrm{PMMT}$ simulations. 


\begin{tabular}{|l||l|l|}
\hline \multicolumn{1}{|l||}{ Distributions } & \multicolumn{2}{|l|}{ Eff. Temperatures (K) } \\
\hline Angle Integrated & s-PMMT & d-PMMT \\
\hline Rotation & 1150 & 873 \\
\hline Vibration & 1150 & 1598 \\
\hline Normal Translation & 1150 & 1450 \\
\hline Surface & 1150 & 1450 \\
\hline
\end{tabular}

\begin{tabular}{|l||l|l|}
\hline $\boldsymbol{\vartheta}=\mathbf{0}^{\circ}$ & s-PMMT & d-PMMT \\
\hline Rotation & 1500 & 873 \\
\hline Vibration & 1500 & 1727 \\
\hline Normal Translation & 1500 & 1600 \\
\hline Surface & 1500 & 1600 \\
\hline
\end{tabular}

\begin{tabular}{|l||l|l|}
\hline $\boldsymbol{\vartheta}=\mathbf{8 9}^{\circ}$ & s-PMMT & d-PMMT \\
\hline Rotation & 1250 & 873 \\
\hline Vibration & 1250 & 1826 \\
\hline Normal Translation & 0 & 0 \\
\hline Surface & 1250 & 2835 \\
\hline
\end{tabular}

Table 3.2. Effective temperatures of the successfully-reacting-reagents state distributions for $873 \mathrm{~K}$ thermal dissociative chemisorption of methane on $\operatorname{Pt}(111)$ as calculated by comparison of the distributions' mean energies to those of Boltzmann distributions. 

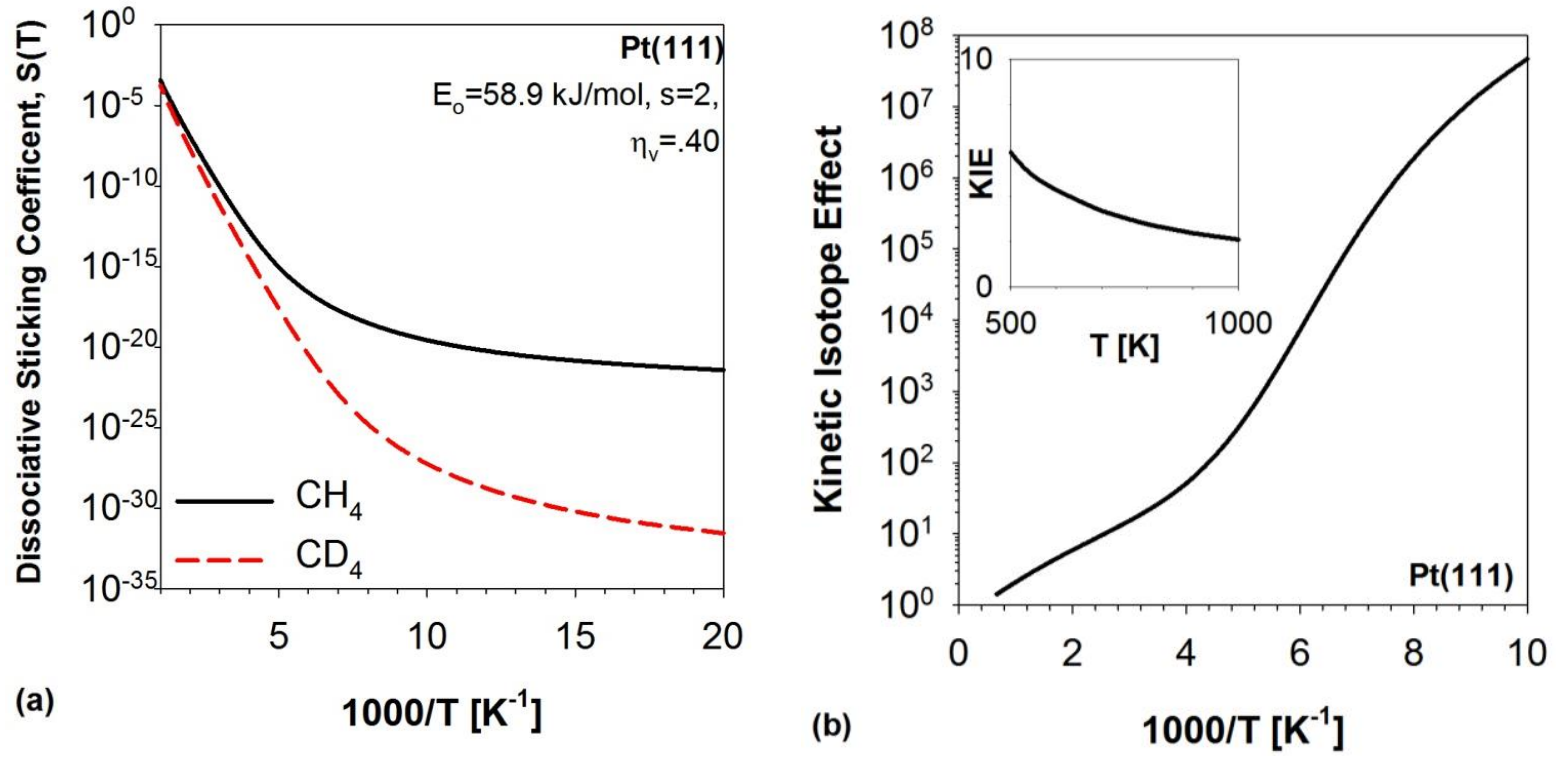

Figure 3.20. Kinetic isotope effect calculated from d-PMMT methane dissociative sticking coefficients on $\mathrm{Pt}(111)$ under thermal conditions. 

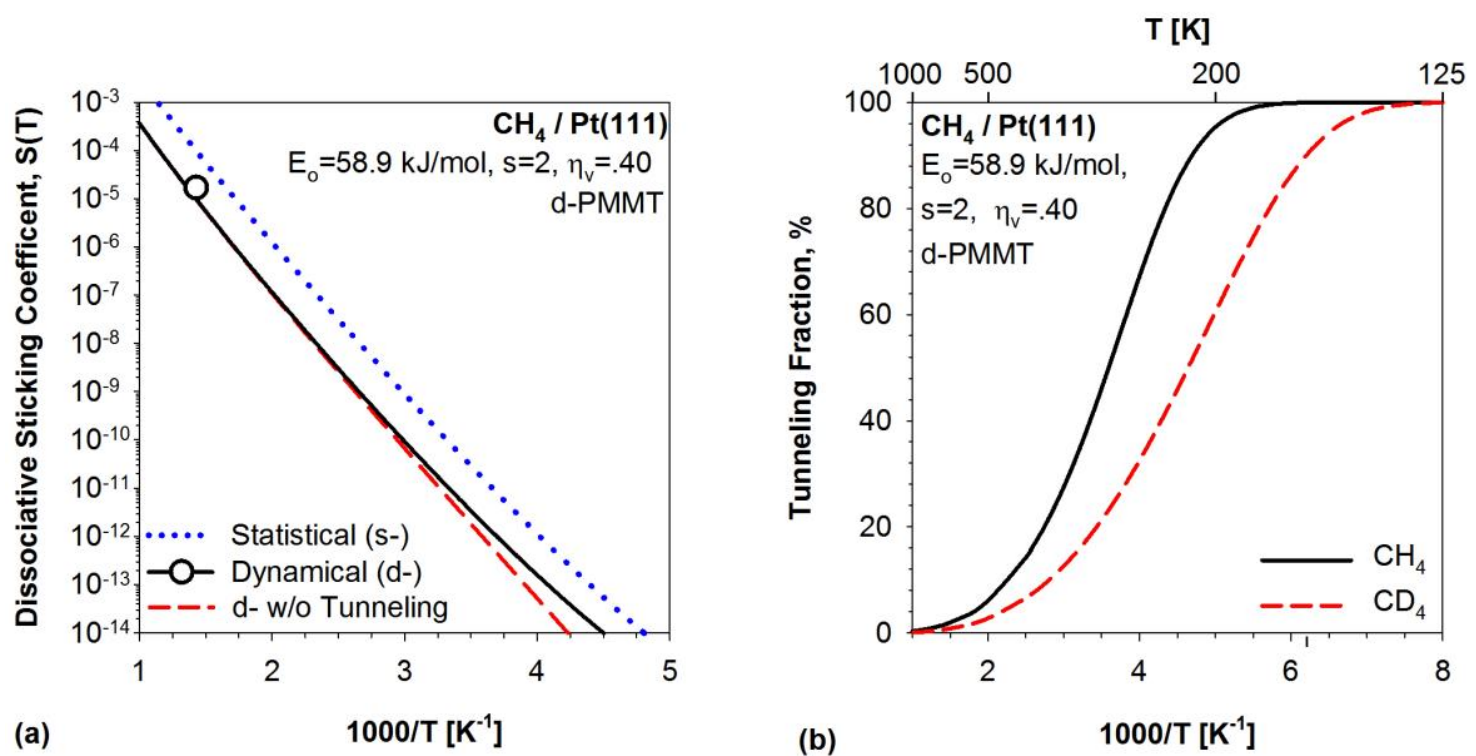

Figure 3.21. (a) Comparison of dissociative sticking coefficients for a thermal ambient $\mathrm{CH}_{4}$ gas in equilibrium with the $\mathrm{Pt}(111)$ surface calculated by d-PMMT simulation with (solid line) and without (dashed line) tunneling through the reaction barrier, and with statistical treatment of the vibrational energy, $\eta_{v} \rightarrow 1$ (dotted line). (b) Relative importance of tunneling in promoting reactivity as a function of temperature in the dissociative chemisorption of methane on $\operatorname{Pt}(111)$. 


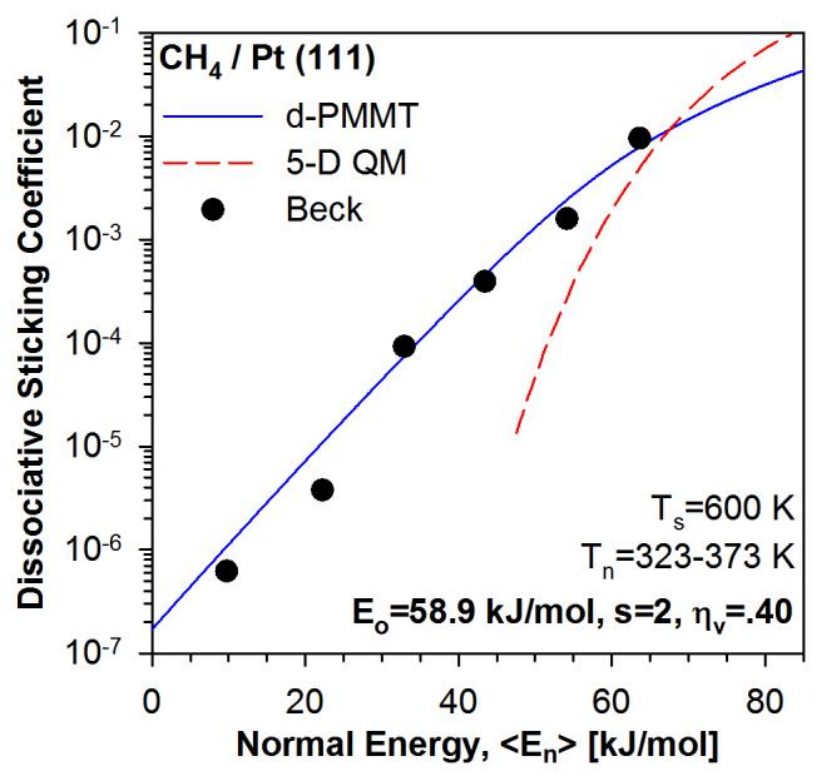

Figure 3.22. Dissociative sticking coefficients for a supersonic molecular beam of $\mathrm{CH}_{4}$ incident on $\mathrm{Pt}(111)$. Beck's thermal nozzle experiments ${ }^{21}$ (points) are compared to a dPMMT simulation and a recent 5-dimensional quantum mechanics (5D-QM) calculation $^{22}$ incorporating one moving surface atom and methane modeled as a pseudodiatomic molecule. 

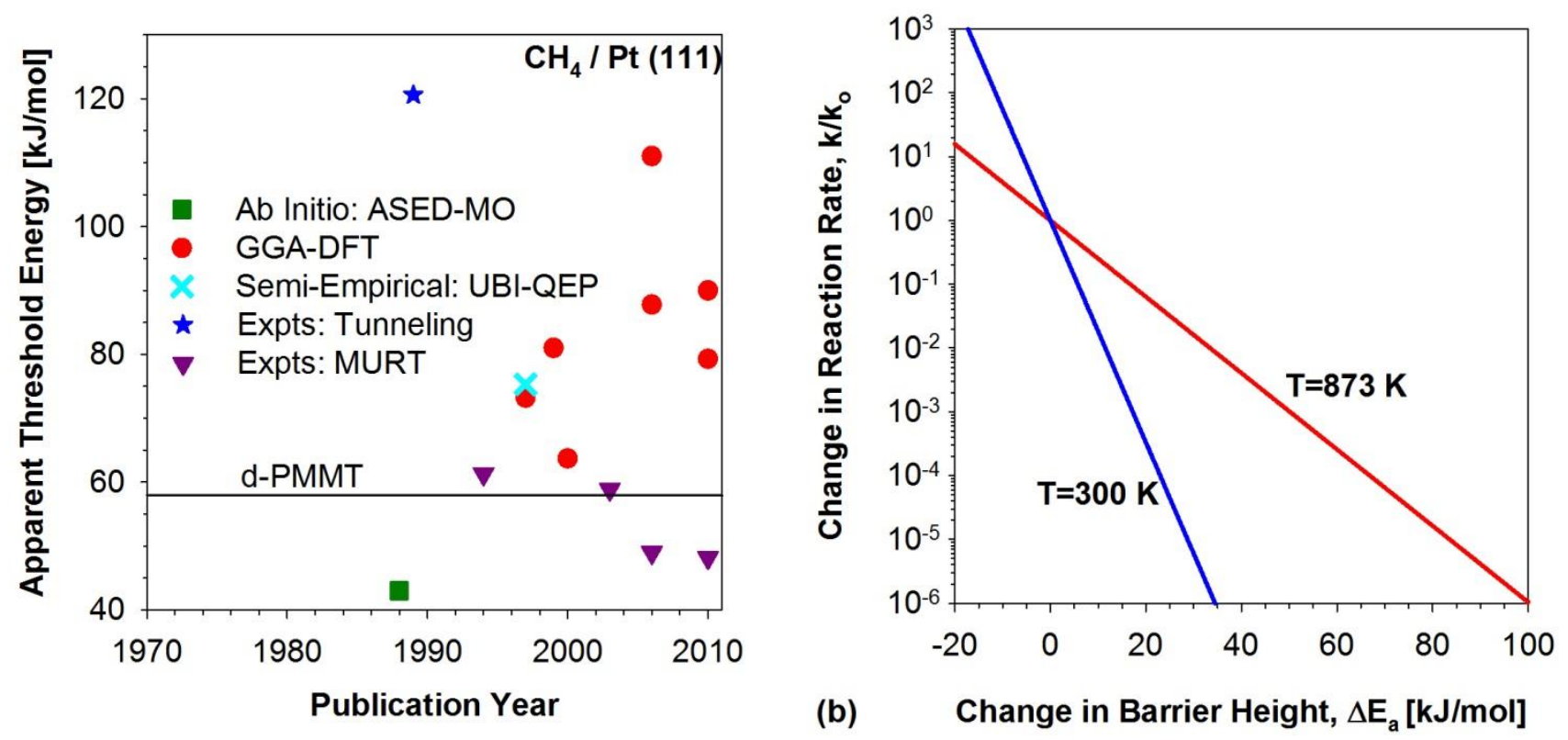

Figure 3.23. (a) Apparent threshold energies for $\mathrm{CH}_{4} / \mathrm{Pt}(111)$ dissociative chemisorption calculated using electronic structure theory techniques ${ }^{39,77-83}$ or kinetic model treatments of various kinds of experimental data. ${ }^{9,15,16,36,38,66}$ (b) Change in rate of thermal reactivity for the difference between d-PMMT barrier height and the range of apparent threshold energies show in (a). 


\begin{tabular}{|l|l|l|l|}
\hline & $v(T S)$ & Normal Mode Description & $v\left(\mathrm{CH}_{4(\mathrm{~g})}\right)$ \\
\hline $\mathrm{E}_{\mathrm{b}}[\mathrm{kJ} / \mathrm{mol}]$ & 89.5 & & -- \\
\hline $\mathrm{f} 1\left[\mathrm{~cm}^{-1}\right]$ & 3126 & $v_{3}:$ Asymmetric C-H Stretch & 3099 \\
\hline $\mathrm{f} 2\left[\mathrm{~cm}^{-1}\right]$ & 3064 & $v_{3}:$ Asymmetric C-H Stretch & 3099 \\
\hline $\mathrm{f} 3\left[\mathrm{~cm}^{-1}\right]$ & 2970 & $v_{3}:$ Asymmetric C-H Stretch & 3097 \\
\hline $\mathrm{f} 4\left[\mathrm{~cm}^{-1}\right]$ & 1633 & $v_{1}:$ Symmetric C-H Stretch & 2980 \\
\hline $\mathrm{f} 5\left[\mathrm{~cm}^{-1}\right]$ & 1389 & $v_{2}:$ Scissor Deformation & 1513 \\
\hline $\mathrm{f} 6\left[\mathrm{~cm}^{-1}\right]$ & 1352 & $v_{2}:$ Scissor Deformation & 1512 \\
\hline $\mathrm{f} 7\left[\mathrm{~cm}^{-1}\right]$ & 1161 & $v_{4}:$ Umbrella Deformation & 1288 \\
\hline $\mathrm{f} 8\left[\mathrm{~cm}^{-1}\right]$ & 832 & $v_{4}:$ Umbrella Deformation & 1288 \\
\hline $\mathrm{f9}\left[\mathrm{cm}^{-1}\right]$ & 813 & $v_{4}:$ Umbrella Deformation & 1287 \\
\hline $\mathrm{f} 10\left[\mathrm{~cm}^{-1}\right]$ & 418 & Perpendicular Translation & -- \\
\hline $\mathrm{f} 11\left[\mathrm{~cm}^{-1}\right]$ & 315 & Rotation & -- \\
\hline $\mathrm{f} 12\left[\mathrm{~cm}^{-1}\right]$ & 130 & Parallel Translation & -- \\
\hline $\mathrm{f} 13\left[\mathrm{~cm}^{-1}\right]$ & 128 & Rotation & -- \\
\hline $\mathrm{f} 14\left[\mathrm{~cm}^{-1}\right]$ & 119 & Rotation & -- \\
\hline $\mathrm{f} 15\left[\mathrm{~cm}^{-1}\right]$ & $953 i$ & Reaction Coordinate & -- \\
\hline
\end{tabular}

Table 3.3: Identification of the normal $\operatorname{modes}^{84}$ associated with the GGA-DFT calculated vibrational frequencies reported for the $\mathrm{CH}_{4} / \mathrm{Pt}(111)$ "D1" transition state (TS) of Table IV of J. Chem. Phys. 132054705 (2010). The electronic energy barrier, $E_{b}=89.5 \mathrm{~kJ} / \mathrm{mol}$, for the D1 transition state along with the GGA-DFT calculated frequencies for methane in the gas-phase are also listed.

The 3 rotations and 3 translations of methane in the gas phase become frustrated motions at the $\mathrm{CH}_{4} / \mathrm{Pt}(111)$ transition state for chemisorption with different vibrational frequencies. Of these frustrated modes, the one corresponding to translation perpendicular to the surface plane stays relatively pure, whereas the five remaining modes ( 3 rotations and 2 parallel translations) can be described as having composite modal properties. The table above associates vibrational normal mode frequencies with descriptors calling out the predominant motion of each mode. The f1-f9 modes evolve primarily from the $v_{1}-v_{4}$ normal modes of gas-phase methane. The f15 reaction coordinate mode has an imaginary frequency and is an admixture of one of the parallel translational modes, some rotation, and a separation of the $\mathrm{CH}_{3}$ and $\mathrm{H}$ product fragments parallel to the surface plane. Because parallel translations were treated as spectator degrees of freedom in the PMMT models, the f12 mode was always removed from the chemisorption transition state. Removal of the second parallel translation mode was always accounted for because it is primarily associated with the separation of products along the reaction coordinate. The modes predominantly associated with rotations (f11, f13, f14) were included or removed from the chemisorption transition state in the accordance with whether the rotations were being treated as active or spectator degrees of freedom. 


\section{References:}

1. Chorkendorff, I.; Niemantsverdriet, J. W., Concepts of Modern Catalysis and Kinetics Wiley-VCH Verlag GmbH \& Co.: Weinheim, 2003.

2. Wei, J. M.; Iglesia, E., Isotopic and kinetic assessment of the mechanism of reactions of $\mathrm{CH}_{4}$ with $\mathrm{CO}_{2}$ or $\mathrm{H}_{2} \mathrm{O}$ to form synthesis gas and carbon on nickel catalysts. J. Catal. 2004, 224, (2), 370-383.

3. Abbott, H. L.; Harrison, I., Methane dissociative chemisorption on $\mathrm{Ru}(0001)$ and comparison to metal nanocatalysts. J. Catal. 2008, 254, (1), 27-38.

4. Jones, G.; Jakobsen, J. G.; Shim, S. S.; Kleis, J.; Andersson, M. P.; Rossmeisl, J.; AbildPedersen, F.; Bligaard, T.; Helveg, S.; Hinnemann, B.; Rostrup-Nielsen, J. R.; Chorkendorff, I.; Sehested, J.; Nørskov, J. K., First principles calculations and experimental insight into methane steam reforming over transition metal catalysts. J. Catal. 2008, 259, (1), 147-160.

5. Durr, M.; Hofer, U., Dissociative adsorption of molecular hydrogen on silicon surfaces. Surf. Sci. Rep. 2006, 61, (12), 465-526.

6. Wei, J. M.; Iglesia, E., Mechanism and site requirements for activation and chemical conversion of methane on supported Pt clusters and turnover rate comparisons among noble metals. J. Phys. Chem. B 2004, 108, (13), 4094-4103.

7. Rauscher, H., The interaction of silanes with silicon single crystal surfaces: microscopic processes and structures. Surf. Sci. Rep. 2001, 42, (6-8), 207-328.

8. Lennard-Jones, J. E., Processes of adsorption and diffusion on solid surfaces. Transactions of the Faraday Society 1932, 28, 333-359.

9. Bukoski, A.; Blumling, D.; Harrison, I., Microcanonical unimolecular rate theory at surfaces. I. Dissociative chemisorption of methane on $\operatorname{Pt}(111)$. J. Chem. Phys. 2003, 118, (2), 843-871.

10. Abbott, H. L.; Bukoski, A.; Harrison, I., Microcanonical unimolecular rate theory at surfaces. II. Vibrational state resolved dissociative chemisorption of methane on $\mathrm{Ni}(100) . J$. Chem. Phys. 2004, 121, (8), 3792-3810.

11. Bukoski, A.; Abbott, H. L.; Harrison, I., Microcanonical unimolecular rate theory at surfaces. III. Thermal dissociative chemisorption of methane on $\operatorname{Pt}(111)$ and detailed balance. $J$. Chem. Phys. 2005, 123, (9), 094707.

12. Abbott, H. L.; Harrison, I., Microcanonical transition state theory for activated gassurface reaction dynamics: Application to $\mathrm{H}_{2} / \mathrm{Cu}(111)$ with rotation as a spectator. J. Phys. Chem. A 2007, 111, (39), 9871-9883.

13. Abbott, H. L.; Harrison, I., Activated dissociation of $\mathrm{CO}_{2}$ on $\mathrm{Rh}(111)$ and $\mathrm{CO}$ oxidation dynamics. J. Phys. Chem. C 2007, 111, (35), 13137-13148.

14. Kavulak, D. F.; Abbott, H. L.; Harrison, I., Nonequilibrium activated dissociative chemisorption: $\mathrm{SiH}_{4}$ on $\mathrm{Si}(100)$. J. Phys. Chem. B 2005, 109, (2), 685-688.

15. Cushing, G. W.; Navin, J. K.; Donald, S. B.; Valadez, L.; Johanek, V.; Harrison, I., C-H Bond Activation of Light Alkanes on Pt(111): Dissociative Sticking Coefficients, Evans-Polanyi Relation, and Gas-Surface Energy Transfer. J. Phys. Chem. C 2010, 114, (40), 17222-17232.

16. Cushing, G. W.; Navin, J. K.; Donald, S. B.; Valadez, L.; Johánek, V.; Harrison, I., Addition/Correction to: C-H Bond Activation of Light Alkanes on Pt(111): Dissociative Sticking Coefficients, Evans-Polanyi Relation, and Gas-Surface Energy Transfer (vol 114, pg 17222, 2010). J. Phys. Chem. C 2010, 114, (51), 22790-22790.

17. Rettner, C. T.; Michelsen, H. A.; Auerbach, D. J., Quantum-State-Specific Dynamics of the Dissociative Adsorption and Associative Desorption of $\mathrm{H}_{2}$ at a $\mathrm{Cu}(111)$ Surface. J. Chem. Phys. 1995, 102, (11), 4625-4641.

18. Juurlink, L. B. F.; Killelea, D. R.; Utz, A. L., State-resolved probes of methane dissociation dynamics. Prog. Surf. Sci. 2009, 84, (3-4), 69-134. 
19. Beck, R. D.; Maroni, P.; Papageorgopoulos, D. C.; Dang, T. T.; Schmid, M. P.; Rizzo, T. R., Vibrational mode-specific reaction of methane on a nickel surface. Science 2003, 302, (5642), 98-100.

20. Smith, R. R.; Killelea, D. R.; DelSesto, D. F.; Utz, A. L., Preference for vibrational over translational energy in a gas-surface reaction. Science 2004, 304, (5673), 992-995.

21. Bisson, R.; Sacchi, M.; Dang, T. T.; Yoder, B.; Maroni, P.; Beck, R. D., State-resolved reactivity of $\mathrm{CH}_{4}\left(2 \mathrm{n}_{3}\right)$ on $\mathrm{Pt}(111)$ and $\mathrm{Ni}(111)$ : Effects of barrier height and transition state location. J. Phys. Chem. A 2007, 111, (49), 12679-12683.

22. Tiwari, A. K.; Nave, S.; Jackson, B., The temperature dependence of methane dissociation on $\mathrm{Ni}(111)$ and $\mathrm{Pt}(111)$ : Mixed quantum-classical studies of the lattice response. $J$. Chem. Phys. 2010, 132, (13), 134702.

23. Yang, K.; Zheng, J. J.; Zhao, Y.; Truhlar, D. G., Tests of the RPBE, revPBE, tauHCTHhyb, omega B97X-D, and MOHLYP density functional approximations and 29 others against representative databases for diverse bond energies and barrier heights in catalysis. $J$. Chem. Phys. 2010, 132, (16), 164117.

24. Balooch, M.; Cardillo, M. J.; Miller, D. R.; Stickney, R. E., Molecular beam study of the apparent activation barrier associated with adsorption and desorption of hydrogen on copper. Surf. Sci. 1974, 46, (2), 358-392.

25. Hodgson, A., State resolved desorption measurements as a probe of surface reactions. Prog. Surf. Sci. 2000, 63, (1-2), 1-61.

26. Michelsen, H. A.; Rettner, C. T.; Auerbach, D. J.; Zare, R. N., Effect of Rotation on the Translational and Vibrational-Energy Dependence of the Dissociative Adsorption of $\mathrm{D}_{2}$ on $\mathrm{Cu}(111)$. J. Chem. Phys. 1993, 98, (10), 8294-8307.

27. Murphy, M. J.; Hodgson, A., Adsorption and desorption dynamics of $\mathrm{H}_{2}$ and $\mathrm{D}_{2}$ on $\mathrm{Cu}(111)$ : The role of surface temperature and evidence for corrugation of the dissociation barrier. J. Chem. Phys. 1998, 108, (10), 4199-4211.

28. Rettner, C. T.; Pfnur, H. E.; Auerbach, D. J., Dissociative Chemisorption of CH4 on W(110) - Dramatic Activation by Initial Kinetic-Energy. Phys. Rev. Lett. 1985, 54, (25), 27162719.

29. Lee, M. B.; Yang, Q. Y.; Ceyer, S. T., Dynamics of the Activated Dissociative Chemisorption of $\mathrm{CH} 4$ and Implication for the Pressure Gap in Catalysis - a Molecular-Beam High-Resolution Electron-Energy Loss Study. J. Chem. Phys. 1987, 87, (5), 2724-2741.

30. Luntz, A. C.; Bethune, D. S., Activation of Methane Dissociation on a Pt(111) Surface. J. Chem. Phys. 1989, 90, (2), 1274-1280.

31. Juurlink, L. B. F.; Smith, R. R.; Utz, A. L., The role of rotational excitation in the activated dissociative chemisorption of vibrationally excited methane on $\mathrm{Ni}(100)$. Faraday Discuss. 2000, 117, 147-160.

32. Donald, S. B.; Harrison, I., Dynamically biased RRKM model of activated gas-surface reactivity: vibrational efficacy and rotation as a spectator in the dissociative chemisorption of $\mathrm{CH}_{4}$ on Pt(111). Phys. Chem. Chem. Phys. 2012, 14, (5), 1784-1796.

33. Cushing, G. W.; Navin, J. K.; Valadez, L.; Johánek, V.; Harrison, I., An effusive molecular beam technique for studies of polyatomic gas-surface reactivity and energy transfer. Rev. Sci. Instr. 2011, 82, 044102.

34. Levine, R. D.; Bernstein, R. B., ENERGY DISPOSAL AND ENERGY CONSUMPTION IN ELEMENTARY CHEMICAL-REACTIONS - INFORMATION THEORETIC APPROACH. Acc. Chem. Res. 1974, 7, (12), 393-400.

35. Remacle, F.; Levine, R. D., The Elimination of Redundant Constraints in Surprisal Analysis of Unimolecular Dissociation and Other Endothermic Processes. J. Phys. Chem. A 2009, 113, (16), 4658-4664. 
36. Ukraintsev, V. A.; Harrison, I., A Statistical-Model for Activated Dissociative Adsorption - Application to Methane Dissociation on Pt(111). J. Chem. Phys. 1994, 101, (2), 1564-1581.

37. Tait, S. L.; Dohnalek, Z.; Campbell, C. T.; Kay, B. D., n-alkanes on Pt(111) and on $\mathrm{C}(0001) / \mathrm{Pt}(111)$ : Chain length dependence of kinetic desorption parameters. J. Chem. Phys. 2006, 125, (23), 234308.

38. Schoofs, G. R.; Arumainayagam, C. R.; Mcmaster, M. C.; Madix, R. J., Dissociative Chemisorption of Methane on Pt(111). Surf. Sci. 1989, 215, (1-2), 1-28.

39. Nave, S.; Tiwari, A. K.; Jackson, B., Methane dissociation and adsorption on Ni(111), Pt(111), Ni(100), Pt(100), and Pt(110)-(1x2): Energetic study. J. Chem. Phys. 2010, 132, (5), 054705 .

40. Forst, W., Unimolecular Reactions: A Concise Introduction. 1st ed.; Cambridge University Press: Cambridge UK, 2003.

41. Zhang, W. Q.; Kawamata, H.; Liu, K. P., CH Stretching Excitation in the Early Barrier F $+\mathrm{CHD}_{3}$ Reaction Inhibits CH Bond Cleavage. Science 2009, 325, (5938), 303-306.

42. Polanyi, J. C.; Wong, W. H., Location of Energy Barriers. I. Effect on the Dynamics of Reactions A + BC. J. Chem. Phys. 1969, 51, 1439-1450.

43. Miller, W. H., Unified statistical-model for complex and direct reaction-mechanisms. $J$. Chem. Phys. 1976, 65, (6), 2216-2223.

44. Diaz, C.; Olsen, R. A., A note on the vibrational efficacy in molecule-surface reactions. $J$. Chem. Phys. 2009, 130, (9), 094706.

45. Nave, S.; Jackson, B., Vibrational mode-selective chemistry: Methane dissociation on Ni(100). Phys. Rev. B 2010, 81, (23), 233408.

46. Miller, W. H., Tunneling corrections to unimolecular rate constants, with application to formaldehyde. J. Am. Chem. Soc. 1979, 101, (23), 6810-6814.

47. Baer, T.; Hase, W. L., Unimolecular Reaction Dynamics. Oxford University Press: New York, NY, 1996.

48. Johnston, H. S.; Heicklen, J., Tunnelling corrections for unsymmetrical Eckart potential energy barriers. J. Phys. Chem. 1962, 66, (3), 532-533.

49. Booze, J. A.; Weitzel, K. M.; Baer, T., The rates of $\mathrm{HCl}$ loss from energy-selected ethylchloride ions - a case of tunneling through an H-atom transfer barrier. J. Chem. Phys. 1991, 94, (5), 3649-3656.

50. Zaera, F.; Chrysostomou, D., Propylene on Pt(111) II. Hydrogenation, dehydrogenation, and H-D exchange. Surf. Sci. 2000, 457, (1-2), 89-108.

51. Zaera, F.; Chrysostomou, D., Propylene on $\operatorname{Pt}(111)$ : I. Characterization of surface species by infra-red spectroscopy. Surf. Sci. 2000, 457, (1-2), 71-88.

52. Zaera, F.; French, C. R., Mechanistic changes in the conversion of ethylene to ethylidyne on transition metals induced by changes in surface coverages. J. Am. Chem. Soc. 1999, 121, (10), 2236-2243.

53. Harris, J.; Simon, J.; Luntz, A. C.; Mullins, C. B.; Rettner, C. T., Thermally Assisted Tunneling - $\mathrm{CH}_{4}$ Dissociation on $\mathrm{Pt}(111)$. Phys. Rev. Lett. 1991, 67, (5), 652-655.

54. Weaver, J. F.; Krzyzowski, M. A.; Madix, R. J., Direct collisionally activated and trapping-mediated dissociative chemisorption of neopentane on clean $\mathrm{Pt}(111)$ : the activity of surface defect sites. Surf. Sci. 1997, 393, (1-3), 150-161.

55. Higgins, J.; Conjusteau, A.; Scoles, G.; Bernasek, S. L., State selective vibrational $\left(2 \mathrm{n}_{3}\right)$ activation of the chemisorption of methane on Pt (111). J. Chem. Phys. 2001, 114, (12), 52775283.

56. Navin, J. K.; Donald, S. B.; Tinney, D. G.; Cushing, G. W.; Harrison, I., Communication: Angle-resolved thermal dissociative sticking of $\mathrm{CH}_{4}$ on $\mathrm{Pt}(111)$ : Further indication that rotation is a spectator to the gas-surface reaction dynamics. J. Chem. Phys. 2012, 136, 061101. 
57. van Willigen, W., Angular Distribution of Hydrogen Molecules Desorbed from Metal Surfaces. Physics Letters 1968, 28A, (2), 80-81.

58. Ukraintsev, V. A.; Harrison, I., Methane Dissociative Chemisorption on $\operatorname{Pt}(111)$ Explored by Microscopic Reversibility. Surf. Sci. 1993, 286, (3), L571-L576.

59. Watanabe, K.; Lin, M. C.; Gruzdkov, Y. A.; Matsumoto, Y., Mechanism for the desorption of molecularly and dissociatively adsorbed methane on $\mathrm{Pt}(111)$ probed by pulse-laser heating. J. Chem. Phys. 1996, 104, (15), 5974-5982.

60. Karp, E. M.; Silbaugh, T. L.; Campbell, C. T., Energetics of Adsorbed CH3 and CH on Pt(111) by Calorimetry: Dissociative Adsorption of CH3I. The Journal of Physical Chemistry C 2013, 117, (12), 6325-6336.

61. Holmblad, P. M.; Wambach, J.; Chorkendorff, I., Molecular-Beam Study of Dissociative Sticking of Methane on Ni(100). J. Chem. Phys. 1995, 102, (20), 8255-8263.

62. Bukoski, A.; Harrison, I., Assessing a microcanonical theory of gas-surface reactivity: Applicability to thermal equilibrium, nonequilibrium, and eigenstate-resolved dissociation of methane on Ni(100). J. Chem. Phys. 2003, 118, (21), 9762-9768.

63. Tolman, R. C., Statistical merchanics applied to chemical kinetics. J. Am. Chem. Soc. 1920, 42, (12), 2506-2528.

64. Luntz, A. C., A simple model for associative desorption and dissociative chemisorption. J. Chem. Phys. 2000, 113, (16), 6901-6905.

65. Diaz, C.; Pijper, E.; Olsen, R. A.; Busnengo, H. F.; Auerbach, D. J.; Kroes, G. J., Chemically Accurate Simulation of a Prototypical Surface Reaction: $\mathrm{H}_{2}$ Dissociation on $\mathrm{Cu}(111)$. Science 2009, 326, (5954), 832-834.

66. DeWitt, K. M.; Valadez, L.; Abbott, H. L.; Kolasinski, K. W.; Harrison, I., Using effusive molecular beams and microcanonical unimolecular rate theory to characterize $\mathrm{CH}_{4}$ dissociation on Pt(111). J. Phys. Chem. B 2006, 110, (13), 6705-6713.

67. Kroes, G. J., Frontiers in surface scattering simulations. Science 2008, 321, (5890), 794797.

68. Hand, M.; Harris, J., Recoil effects in surface dissociation. J. Chem. Phys. 1990, 92, (12), 7610-7617.

69. Wang, Z. S.; Darling, G. R.; Holloway, S., Surface temperature dependence of the inelastic scattering of hydrogen molecules from metal surfaces. Phys. Rev. Lett. 2001, 87, (22), 226102.

70. Dahl, S.; Logadottir, A.; Egeberg, R. C.; Larsen, J. H.; Chorkendorff, I.; Tornqvist, E.; Norskov, J. K., Role of steps in N2 activation on Ru(0001). Phys. Rev. Lett. 1999, 83, (9), 18141817.

71. Luntz, A. C., The dynamics of making and breaking bonds at surfaces. In Chemical bonding at surfaces and interfaces, Nilsson, A.; Pettersson, L.; Norskov, J. K., Eds. Elsevier: 2008; pp 143-254.

72. Jones, G.; Jakobsen, J. G.; Shim, S. S.; Kleis, J.; Andersson, M. P.; Rossmeisl, J.; AbildPedersen, F.; Bligaard, T.; Helveg, S.; Hinnemann, B.; Rostrup-Nielsen, J. R.; Chorkendorff, I.; Sehested, J.; Norskov, J. K., First principles calculations and experimental insight into methane steam reforming over transition metal catalysts. J. Catal. 2008, 259, (1), 147-160.

73. Ligthart, D.; van Santen, R. A.; Hensen, E. J. M., Influence of particle size on the activity and stability in steam methane reforming of supported Rh nanoparticles. J. Catal. 2011, 280, (2), 206-220.

74. Sutter, E.; Albrecht, P.; Camino, F. E.; Sutter, P., Monolayer graphene as ultimate chemical passivation layer for arbitrarily shaped metal surfaces. Carbon 2010, 48, (15), 44144420 .

75. Bae, S.; Kim, H.; Lee, Y.; Xu, X. F.; Park, J. S.; Zheng, Y.; Balakrishnan, J.; Lei, T.; Kim, H. R.; Song, Y. I.; Kim, Y. J.; Kim, K. S.; Ozyilmaz, B.; Ahn, J. H.; Hong, B. H.; Iijima, S., 
Roll-to-roll production of 30-inch graphene films for transparent electrodes. Nature Nanotechnology 2010, 5, (8), 574-578.

76. Matsumoto, Y.; Gruzdkov, Y. A.; Watanabe, K.; Sawabe, K., Laser-induced photochemistry of methane on $\mathrm{Pt}(111)$ : Excitation mechanism and dissociation dynamics. $J$. Chem. Phys. 1996, 105, (11), 4775-4788.

77. Anderson, A. B.; Maloney, J. J., Activation of Methane on Iron, Nickel, and Platinum Surfaces - a Molecular-Orbital Study. J. Phys. Chem. 1988, 92, (3), 809-812.

78. Liao, M. S.; Au, C. T.; Ng, C. F., Methane dissociation on Ni, Pd, Pt and Cu metal(111) surfaces - A theoretical comparative study. Chem. Phys. Lett. 1997, 272, (5-6), 445-452.

79. Shustorovich, E.; Sellers, H., The UBI-QEP method: a practical theoretical approach to understanding chemistry on transition metal surfaces. Surf. Sci. Rep. 1998, 31, (1-3), 5-119.

80. Au, C. T.; Ng, C. F.; Liao, M. S., Methane dissociation and syngas formation on Ru, Os, Rh, Ir, Pd, Pt, Cu, Ag, and Au: A theoretical study. J. Catal. 1999, 185, (1), 12-22.

81. Michaelides, A.; Hu, P., Insight into microscopic reaction pathways in heterogeneous catalysis. J. Am. Chem. Soc. 2000, 122, (40), 9866-9867.

82. Psofogiannakis, G.; St-Amant, A.; Ternan, M., Methane oxidation mechanism on Pt(111): A cluster model DFT study. J. Phys. Chem. B 2006, 110, (48), 24593-24605.

83. Vines, F.; Lykhach, Y.; Staudt, T.; Lorenz, M. P. A.; Papp, C.; Steinruck, H. P.; Libuda, J.; Neyman, K. M.; Gorling, A., Methane Activation by Platinum: Critical Role of Edge and Corner Sites of Metal Nanoparticles. Chemistry-a European Journal 2010, 16, (22), 6530-6539. 84. Nave, S.; Jackson, B., personal communication 2010. 
4.

\title{
RRKM Simulation of Methane Dissociation on Ru(0001), $\mathrm{Ni}(111), \mathrm{Ni}(100)$ and $\operatorname{Ir}(111)$ :
}

\section{Connecting d-PMMT Parameters to Electronic Structure Theory}

\begin{abstract}
The reactivity of methane gas on different single crystal transition metal surfaces was compared using a dynamically biased precursor mediated microcanonical trapping (d-PMMT) model to analyze experimental data. The model took molecular translational energy parallel to the surface and rotations to be spectator degrees of freedom and a dynamical bias was imposed on the ability of vibration to contribute active exchangeable energy capable of surmounting the barrier to dissociative chemisorption. The d-PMMT was used to simulate dissociative sticking coefficients for a broad range of experiments that included disparate supersonic beam, eigenstate resolved, and thermal bulb meansurements, for $\mathrm{Ru}(0001), \mathrm{Ni}(111), \mathrm{Ni}(100)$, and $\operatorname{Ir}(111)$. Average relative discrepancies (ARDs) between d-PMMT simulations and experiments were relatively good, with overall ARDs of $185 \%, 177 \%, 190 \%$, and $156 \%$ for the earlier list of surfaces. At catalytically relevant temperatures, surface phonons were found to play an important role in promoting reactivity on all surfaces. GGA-DFT calculations were used to provide transition state vibrational frequencies. Relationships between the transition state properties calculated using electronic structure theory and experimentally derived d-PMMT parameters were explored.
\end{abstract}




\subsection{Introduction}

The importance of steam reforming of methane, a process performed industrially, on a multi-billion dollar per year scale, where $\mathrm{CH}_{4}$ and steam react over supported nickel nanocatalysts as the most economic method for producing $\mathrm{H}_{2}$ and synthesis gas, has encouraged many people to seek a greater understanding of methane dissociative chemisorption. Experimentally, it has been claimed that the initial C-H bond breaking is the rate limiting step in the catalytic decomposition of methane. ${ }^{1-6}$ Although the bond dissociation energy decreases from $432 \mathrm{~kJ} / \mathrm{mol}$ in the gas phase ${ }^{7}$ to less than $100 \mathrm{~kJ} / \mathrm{mol}$ when performed over a nickel catalyst, even lower activation energies would be preferable to reduce the operating temperature of methane reforming. Many transition metal surfaces have been experimentally and theoretically explored as potential candidates for improved methane dissociation including $\operatorname{Pt}(111),{ }^{8}, 9 \operatorname{Ir}(111),{ }^{10,}{ }^{11}$ $\operatorname{Rh}(111),{ }^{12,13} \mathrm{Ni}(111),{ }^{14-16} \mathrm{Ni}(100),{ }^{14,}{ }^{17-28} \mathrm{Pd}(111),{ }^{29-31}$ and $\mathrm{Ru}(0001){ }^{32-35}$. Figure 4.1 depicts the various activation energies $\left(\mathrm{E}_{\mathrm{a}}\right)$ and reaction threshold energies $\left(\mathrm{E}_{0}=\mathrm{E}_{\mathrm{a}}\right.$ at $\left.\mathrm{T}=0 \mathrm{~K}\right)$ over the past twenty five years for methane dissociative chemisorption on $\operatorname{Ir}(111), \operatorname{Ru}(0001), \mathrm{Ni}(100)$, and $\mathrm{Ni}(111)$ derived from a range of experimental data ${ }^{26,33-45}$ or electronic structure theory calculations. ${ }^{14,34,46-61}$ Improved detection methods and the implementation of the thermal finger technique in thermal bulb experiments ${ }^{8}$ have led to more accurate experimental measurements and typically increasing values reaction the activation energies over time. Density functional theory (DFT) predictions vary widely depending on the method and functional chosen for calculations.

\subsection{A. $\mathrm{Ni}(100) \& \mathrm{Ni}(111)$}

Studies of the activated dissociative chemisorption of methane on $\mathrm{Ni}(100)^{14,17,19-28}$ and $\mathrm{Ni}(111)^{14,23,42,58,62-71}$ serve as important model reactions that provide insight into steam reforming of methane over Ni catalysts. ${ }^{72}$ A diverse range of experimental studies and theoretical calculations are available, making methane single crystal reactivity an excellent test-bed for 
evaluating emerging models of polyatomic gas-surface reactivity. The dissociative chemisorption of methane on metals has at times been proposed to be dominated by a tunneling ${ }^{9,73-76}$ pathway or by translational or vibrational energy induced deformations of methane,${ }^{77,78}$ but is currently held to proceed in a standard over-the-barrier mechanism. ${ }^{37,79,80}$

Eigenstate resolved molecular beam experiments indicate significant enhancement in the dissociative sticking coefficients as vibrational and translational energy are independently increased for $\mathrm{CH}_{4}$ impinging on $\mathrm{Ni}(100){ }^{20,} 58,81$ Effective activation energies have been extrapolated from these molecular beam experiments and could, to some extent, be replicated by a semi-empirical error function (erf) dissociative sticking coefficient model with three adjustable parameters used for each molecular eigenstate in a reduced dimensionality analysis. Rotational cooling within supersonic molecular beams makes it difficult to study the effect of rotational energy on dissociative sticking coefficients. While the error function sticking anzats has sufficient parameterization to fit some molecular beam data adequately well, the parameters appear to have little physical significance or uniqueness and do not confer an ability to predict dissociative sticking coefficients under different experimental conditions. The need to define three parameters for each eigenstate, precludes an erf model from simulating thermal dissociative chemisorption where contributions to the dissociative sticking from $\sim 1400$ eigenstates must be summed to reach just $95 \%$ of the thermal sticking coefficient at $\mathrm{T}=1000 \mathrm{~K} .^{18}$

Semi-empirical erf analysis ${ }^{82}$ of experiments has shown vibrational energy has an efficacy for promoting reactivity of methane dissociative chemisorption on $\mathrm{Ni}(100)$ relative to normal translational energy of about one. It was initially assumed only the asymmetric $v_{3}$ stretching mode is active in methane dissociative chemisorption, from which extrapolations of molecular beam experiments to the catalytic thermal equilibrium conditions, led to predictions that methane vibrationally excited to $v_{3}=1$ dominates the thermal dissociative sticking on $\mathrm{Ni}(100)^{28}$ as compared to $\mathrm{Ru}(0001)$ where the ground state was thought to be dominant. ${ }^{34}$ 
However, eigenstate-resolved studies of methane dissociative chemisorption on $\mathrm{Ni}(100)^{27}$ and $\mathrm{Ni}(111)^{58}$ established that other modes with unique mode-specific vibrational efficacies contribute to the activated sticking observed in molecular beam ${ }^{28}$ and thermal catalysis ${ }^{19}$ experiments as well. Alternatively, activation energies were determined directly from Arrhenius fits of dissociative sticking coefficients measured in thermal equilibrium "bulbs" at pressures in the few millibar range. The most recent thermal equilibrium bulb data yields an activation energy of $59 \mathrm{~kJ} / \mathrm{mol}$ for $\mathrm{CH}_{4} / \mathrm{Ni}(100)^{19}$ and $74.5 \mathrm{~kJ} / \mathrm{mol}$ for $\mathrm{CH}_{4} / \mathrm{Ni}(111)^{42}$ [although lower values of 26.8 and $51.9 \mathrm{~kJ} / \mathrm{mol}$, respectively, were measured earlier]. ${ }^{45}$ These experimental $\mathrm{E}_{\mathrm{a}} \mathrm{s}$ compare to $67 \pm$ $4 \mathrm{~kJ} / \mathrm{mol}$ from ab initio calculations for $\mathrm{CH}_{4} / \mathrm{Ni}(100)^{83}$, and range from $87.6^{14}$ to $103^{26} \mathrm{~kJ} / \mathrm{mol}$ for $\mathrm{CH}_{4} / \mathrm{Ni}(111)$ calculated using density functional theory (DFT) methods.

Several theoretical studies using low-dimensionality potential energy surfaces have suggested methane dissociative chemisorption on $\mathrm{Ni}(100)$ reacts preferentially from favored vibrational $^{84,85}$ or rotational ${ }^{65}$ quantum states. However, a three-parameter, physisorbed complex (PC) microcanonical unimolecular rate theory (MURT) $)^{18,38,86}$ based on transition state theory has been successfully applied to $\mathrm{CH}_{4} / \mathrm{CD}_{4}$ on $\mathrm{Ni}(100)^{87}$ and was able to semi-quantitatively predict a majority of the dissociative sticking of eigenstate-resolved ${ }^{24,27}$, thermal ${ }^{28}$ molecular beam, and mbar pressure thermal equilibrium "bulb" experiments ${ }^{19}$ arguing for a statistical treatment of all active forms of energy (n.b., parallel translation remains a spectator degree of freedom) in methane dissociative chemisorption on nickel.

\subsection{B. $\operatorname{Ru}(0001)$}

The dissociative chemisorption of methane on ruthenium surfaces has been examined using a variety of UHV and catalysis experimental methods. Thermal dissociative sticking coefficients, $\mathrm{S}(\mathrm{T})$, for $\mathrm{CH}_{4}$ on $\mathrm{Ru}(0001)$ were measured under high pressure conditions (i.e., $~ 5$ torr; 11 orders of magnitude higher pressure than traditional UHV experiments) by Wu and Goodman. ${ }^{33}$ An Arrhenius fit of the $S(T)$ data indicated that the activation energy was $36 \mathrm{~kJ} / \mathrm{mol}$ 
in the $T=500-650 \mathrm{~K}$ temperature range experimentally probed. Separate thermal dissociative sticking measurements were made by Egeberg et al. ${ }^{32}$ using thermal "bulb" techniques ( 4 torr). The experimental $S(T)$ values measured by Egeberg et al. ${ }^{32}$ were of the same order of magnitude as those previously reported by $\mathrm{Wu}$ and Goodman. ${ }^{33}$ However, the activation energies differed by approximately $15 \mathrm{~kJ} / \mathrm{mol}$, with Egeberg et al. ${ }^{32}$ reporting a higher activation energy of $E_{a}=51$ $\pm 6 \mathrm{~kJ} / \mathrm{mol}$. Nonetheless, methane dissociative sticking coefficients on $\mathrm{Ru}(0001)$ were found to be approximately an order of magnitude larger than those measured on $\mathrm{Ni}(111)$. By working at lower pressures, in the high $10^{-3}$ Torr range, Egeberg et al. ${ }^{32}$ obtained methane $S\left(T_{g}=300 K ; T_{s}\right)$ for gas incident over a wide range of surface temperatures and determined an effective activation energy " $E_{a}\left(T_{s}\right)$ " $=40 \mathrm{~kJ} / \mathrm{mol}$ using an Arrhenius fit versus surface temperature. Step blocking experiments performed by Egeberg et al. ${ }^{32}$ indicated that steps sites do not observably increase the dissociation of methane as compared to terraces sites, which is very different than the case for $\mathrm{N}_{2}$ dissociative chemisorption on $\mathrm{Ru}(0001)^{88,89}$.

Catalysis experiments ${ }^{90}$ measuring the dissociative chemisorption of methane over oxide supported ruthenium nanoparticles (i.e., $6 \mathrm{~nm}$ average $\mathrm{Ru}$ particle diameter; $\mathrm{Ru} / \mathrm{SiO}_{2}$ ) were found to have sticking coefficients $10^{3}-10^{4}$ lower than those reported by $\mathrm{Wu}$ and Goodman ${ }^{33}$ and Egeberg et al on $\mathrm{Ru}(0001){ }^{32}$ Moreover, an activation energy of $25-29 \mathrm{~kJ} / \mathrm{mol}$ was determined for the $\mathrm{Ru} / \mathrm{SiO}_{2}$ nanocatalysts, approximately $10 \mathrm{~kJ} / \mathrm{mol}$ lower than the $E_{a}$ reported by $\mathrm{Wu}$ and Goodman. ${ }^{33}$ More recently, kinetics, isotopic tracer, and exchange measurements performed by Wei and Iglesia $^{3}$ measured turnover rates for methane decomposition on ruthenium nanoparticles deposited on an alumina support (i.e., $3 \mathrm{~nm}$ average $\mathrm{Ru}$ particle diameter; $\mathrm{Ru} / \mathrm{Al}_{2} \mathrm{O}_{3}$ ). These catalytic turnover rates correspond to equivalent dissociative sticking coefficients which are also three to four orders of magnitude lower than experimental values for $\mathrm{CH}_{4}$ dissociative chemisorption on single crystal $\mathrm{Ru}(0001)$. A large activation energy of $99 \mathrm{~kJ} / \mathrm{mol}$ was reported by Wei and Iglesia ${ }^{3}$ for the decomposition of methane on these Ru nanoparticle catalysts, and a small 
kinetic isotope effect 1.40-1.51 was derived from the isotopic studies they performed. Furthermore, Wei and Iglesia $^{3}$ demonstrated that substrate effects do not influence the experimentally observed $E_{a}$ for methane decomposition on nanoparticle catalysts, but increasing the dispersion does augment the catalyst turnover rates.

The nonequilibrium supersonic molecular beam experiments of Larsen et al. ${ }^{34}$ explored the role of translational and vibrational energy in the dissociation of methane on $\operatorname{Ru}(0001)$. In these experiments, the dissociative sticking coefficients $S\left(E_{n}\right)$ were observed to increase with increasing nozzle temperature $T_{n}$, which is assumed to directly define the molecular vibrational temperature $T_{v}$ (n.b., with negligible vibrational cooling in the supersonic expansion, $T_{v}=T_{n}$ ). By varying the nozzle temperature and the seeding mixture of the methane gas, Larsen et al. ${ }^{34}$ changed the normal translational energy $E_{n}$ of the molecular beam and observed that $S\left(E_{n}\right)$ also increased with increasing normal translational energy. Unfortunately, the role of rotational energy could not be probed in these supersonic molecular beam experiments because of the efficient collision induced cooling of the rotational degrees of freedom that occurs in the supersonic expansion (i.e., $\left.T_{r o t} \approx 0.1 T_{N}\right)^{28}$

Larsen et al. ${ }^{34}$ optimized a nine-parameter empirical error function (“erf") model to fit their nonequilibrium supersonic molecular beam data $S\left(E_{n}\right)$ and predicted thermal sticking coefficients $S(T)$. These "erf" predicted thermal sticking coefficients were then fit to an Arrhenius equation and were found to have an activation energy of $E_{a}=37 \mathrm{~kJ} / \mathrm{mol}$. Mortensen et al. ${ }^{35}$ also measured dissociative sticking coefficients for $\mathrm{CH}_{4}$ on $\mathrm{Ru}(0001)$ using a supersonic molecular beam. They observed that the sticking coefficients increased with increasing normal translational energy $E_{n}$ and with increasing surface temperature $T_{s}$. The surface temperature dependence diminished as the normal translational energy of the supersonic molecular beam increased, showing the enhanced importance of the surface in providing exchangeable energy to surmount the barrier to reactivity under otherwise energy starved conditions. 
Dissociative sticking coefficients $S\left(E_{n}\right)$ obtained from nonequilibrium supersonic molecular beam experiments ${ }^{35}$ for two isotopomers of methane (i.e., $\mathrm{CH}_{4}$ and $\mathrm{CD}_{4}$ ) indicated a kinetic isotope effect (KIE) for methane on $\mathrm{Ru}(0001)$ of approximately $20 .{ }^{35}$ Mortensen et al. ${ }^{35}$ adapted the "erf" model used by Larsen et al. ${ }^{28}$ by incorporating surface temperature dependence into one of the adjustable parameters and then used the updated "erf" model to fit their supersonic molecular beam data ${ }^{35}$ as well as the thermal equilibrium (i.e., $T_{g}=T_{s}$ ) and thermal nonequilibrium (i.e., $T_{g}=300 \mathrm{~K} ; T_{g} \neq T_{s}$ ) experimental data of Larsen et al. ${ }^{28}$

Generalized gradient approximation - density functional theory (GGA-DFT) electronic structure calculations and a unity bond index-quadratic exponential potential (UBI-QEP) method have been used to estimate activation energies for methane dissociation on ruthenium. GGA-DFT computations by Ciobica et al. ${ }^{56}$ suggested that the rate limiting step for methane dissociation on $\mathrm{Ru}(0001)$ is breaking the final $\mathrm{C}-\mathrm{H}$ bond of methylidyne (i.e., $E_{a}=108 \mathrm{~kJ} / \mathrm{mol}$ ) rather than the first $\mathrm{C}-\mathrm{H}$ bond of methane (i.e., $E_{a}=85 \mathrm{~kJ} / \mathrm{mol}$ ). Liu and $\mathrm{Hu}^{53}$ have performed GGA-DFT calculations similar to those completed by Ciobica et al. ${ }^{56}$ for $\mathrm{CH}_{4}$ dissociative chemisorption on $\mathrm{Ru}(0001)$ and found a somewhat lower reaction threshold energy of $76 \mathrm{~kJ} / \mathrm{mol}$. Using UBI-QEP methods, Au et al. ${ }^{54}$ calculated an activation energy of $E_{a}=60 \mathrm{~kJ} / \mathrm{mol}$ for $\mathrm{CH}_{4(\mathrm{c})} \rightarrow \mathrm{CH}_{3(\mathrm{c})}+\mathrm{H}_{(\mathrm{c})}$ on a 10 atom ruthenium cluster, the lowest computed for this process of the investigated transition metals (i.e., $\mathrm{Ag}, \mathrm{Au}, \mathrm{Cu}, \mathrm{Ir}, \mathrm{Os}, \mathrm{Pd}, \mathrm{Rh}$ ). Au et al. ${ }^{54}$ also computed the subsequent $\mathrm{C}-\mathrm{H}$ bond cleavages to give complete dissociation of $\mathrm{CH}_{4}$ to carbon and hydrogen atoms, and they reported that each step required more energy than the previous step with methylidyne dissociation (i.e., $\mathrm{CH}_{(\mathrm{c})} \rightarrow \mathrm{C}_{(\mathrm{c})}+\mathrm{H}_{(\mathrm{c})}$ ) having the highest activation energy (i.e., $E_{a}=115 \mathrm{~kJ} / \mathrm{mol}$ ). Additional UBIQEP calculations ${ }^{49}$ for $\mathrm{CH}_{4}$ dissociative chemisorption on several single crystal transition metal surfaces $[\operatorname{Pt}(111), \operatorname{Rh}(111), \operatorname{Ir}(111), \mathrm{Ni}(111), \mathrm{Ru}(0001)$, and $\mathrm{Cu}(111)]$ found that the activation energy for $\mathrm{CH}_{4}$ dissociation was lowest on $\mathrm{Ru}(0001)$ (i.e., $E_{a}=5.8 \mathrm{~kJ} / \mathrm{mol}$ ). Lin et al. ${ }^{49}$ computed an activation energy of $E_{a}=47 \mathrm{~kJ} / \mathrm{mol}$ for the initial C- $\mathrm{H}$ bond cleavage and found that 
breaking the second and third $\mathrm{C}-\mathrm{H}$ bonds required more energy (ca. $E_{a} \sim 92 \mathrm{~kJ} / \mathrm{mol}$ for $\mathrm{CH}_{3(\mathrm{c})} \rightarrow$ $\mathrm{CH}_{2(\mathrm{c})}+\mathrm{H}_{(\mathrm{c})}$ and $\left.\mathrm{CH}_{2(\mathrm{c})} \rightarrow \mathrm{CH}_{(\mathrm{c})}+\mathrm{H}_{(\mathrm{c})}\right)$, bolstering the argument for the application of detailed balance to dissociative chemisorption of methane on $\mathrm{Ru}(0001)$. Taken as a whole, these electronic structure calculations fail to identify a common activation energy or rate determining step for methane dissociation on $\mathrm{Ru}(0001)$.

\subsection{C. $\operatorname{Ir}(111)$}

Wei and Iglesia claim that $\mathrm{C}-\mathrm{H}$ bond activation is the sole kinetically relevant step in the dry or steam reforming of methane because the rate depends only on the ambient methane pressure. $^{1,91-94}$ Although nickel is the primary catalyst used in industry, iridium has been noted for its high activity. Many values have been proposed as the activation energy for the dissociative chemisorption of methane on $\operatorname{Ir}(111)$. Seets et al. ${ }^{10}$ and Jachimowski et al. ${ }^{11}$ have reported effective activation energies of " $E_{a} "=27 \pm 4 \mathrm{~kJ} / \mathrm{mol}$ and " $E_{a}$ " $=53 \mathrm{~kJ} / \mathrm{mol}$, respectively, which were obtained by fitting an Arrhenius equation to thermal nonequilibrium dissociative sticking coefficient data $\mathrm{S}\left(\mathrm{T}_{\mathrm{g}}=300 \mathrm{~K} ; \mathrm{T}_{\mathrm{s}}\right)$ for an ambient $300 \mathrm{~K}$ gas. Jachimowski et al. also reported a thermal activation energy of $E_{a}=72 \mathrm{~kJ} / \mathrm{mol}$ for $\mathrm{S}(\mathrm{T})$ measured in a few mbar thermal bulb experiment, ${ }^{11}$ while recent high pressure catalysis experiments performed by Wei and Iglesia on Ir nanoparticles indicate a thermal activation energy of $E_{a}=81 \mathrm{~kJ} / \mathrm{mol} .^{94}$ Density functional theory (DFT) calculations performed by Henkelman and Jónsson ${ }^{95}$ and Au et al. ${ }^{49}$ yield (zeropoint corrected) activation energies of $E_{a}=15 \pm 10 \mathrm{~kJ} / \mathrm{mol}$ and $76 \mathrm{~kJ} / \mathrm{mol}$, respectively. The wide range of suggested values from 15 to $81 \mathrm{~kJ} / \mathrm{mol}$ makes it difficult to divine the true activation energy for this reaction.

\section{2. d-PMMT Model}

The specifics of the d-PMMT model have been described previously in Chapters 2 and 3. Briefly, gas-surface collision complexes formed from an incoming molecule and a few local 
surface oscillators with a pooled energy sufficient to react are assumed to have their energy microcanonically randomized through state-mixing collisions on the reactive potential ${ }^{37}$ and/or intramolecular vibrational energy redistribution (IVR) ${ }^{38}$ such that the molecules become trapped in the precursor physisorption well located between the transition states for reaction (dissociative chemisorption) and desorption. These precursor complexes (PCs) go on to react or desorb with Rice-Ramsperger-Kassel-Marcus (RRKM) rate constants,

$$
k_{i}(E)=\frac{W_{i}^{\dagger}\left(E-E_{i}\right)}{h \rho(E)}
$$

where $E$ is the active exchangeable energy, $W_{i}^{\dagger}\left(E-E_{i}\right)$ is the sum of states for transition state $i=D, R$ with threshold energy for reaction $E_{i}$ (e.g., $E_{R}=E_{D}+E_{0}$ in Fig. 3.1), $\rho(E)$ is the PC density of states, and $h$ is Planck's constant. Tunneling is implemented in the reactive sum of states in the manner discussed previously. In the following equations and calculations it is convenient to use the $E^{*}=E-E_{D}$ energy scale whose zero occurs for well separated reactants in their ground states.

Applying the steady state approximation to the simplified Fig. 4.2 kinetics scheme, ${ }^{37,38}$

$$
\mathrm{CH}_{4(g)} \underset{k_{D}\left(E^{*}\right)}{\stackrel{F_{0} f\left(E^{*}\right)}{\longrightarrow}} \mathrm{CH}_{4(p)} \stackrel{k_{R}\left(E^{*}\right)}{\longrightarrow} \mathrm{CH}_{3(c)}+\mathrm{H}_{(c)}
$$

yields the following expression for the experimental dissociative sticking coefficient,

$$
S=\int_{0}^{\infty} S\left(E^{*}\right) f\left(E^{*}\right) d E^{*}
$$

where $S\left(E^{*}\right)$ is the microcanonical sticking coefficient, 


$$
S\left(E^{*}\right)=\frac{k_{R}\left(E^{*}\right)}{k_{R}\left(E^{*}\right)+k_{D}\left(E^{*}\right)}=\frac{W_{R}^{\dagger}\left(E^{*}-E_{0}\right)}{W_{R}^{\dagger}\left(E^{*}-E_{0}\right)+W_{D}^{\dagger}\left(E^{*}\right)}
$$

and $f\left(E^{*}\right)$ is the probability distribution,

$f\left(E^{*}\right)=\int_{0}^{E^{*}} f_{n}\left(E_{n}\right) \int_{0}^{E^{*}-E_{n}} f_{s}\left(E_{s}\right) f_{v}\left(\eta_{v}^{-1}\left(E^{*}-E_{n}-E_{s}\right)\right) d E_{s} d E_{n}$

for forming a PC with energy $E^{*}$ which is calculated by convolution over the molecular and surface energy distributions describing the particular experimental conditions of interest. The experimental sticking coefficient is the average of the microcanonical sticking coefficient over the experimental probability of forming a PC with energy $E^{*}$.

Dissociative sticking coefficients for $\mathrm{CH}_{4}$ on transition metals are experimentally ${ }^{8,} 36$ found to scale with the normal component of the molecular translational energy, or normal translational energy, $E_{n}=E_{t} \cos ^{2} \vartheta$, where $\vartheta$ is the molecular angle of incidence to the surface measured away from the surface normal. In consequence, molecular translational energy parallel to the surface, along with rotations, are taken to be a spectator degree of freedom. Energy associated with all other molecular degrees of freedom and the energy of $s$ surface oscillators is assumed to be active exchangeable energy within the PCs formed. Dissociative sticking calculations require specification of the transition states for desorption and reaction. The desorption transition state was taken to occur when the alkane is freely rotating and vibrating in the gas-phase, far from the $s$ surface oscillators of the united PC. The surface oscillators are assumed to vibrate at the mean phonon frequency of the metal $\left[v_{s}=\left(\frac{3}{4}\right) k_{B} T_{\text {Debye }} / h\right]$ with only the component of the lattice vibrations normal to the surface contributing to the PC exchangeable energy (symmetric with the experimental finding that the dissociative sticking coefficient scales with only the normal component of the molecular translational energy). The desorption 
coordinate was taken to be the vibrational motion that ultimately becomes free molecular translation along the surface normal, and so that degree of freedom is missing from the desorption transition state (n.b., also missing are the spectator translational motions parallel to the surface). The transition state for dissociative chemisorption was defined by the vibrational frequencies calculated by Nave, Tiwari, and Jackson ${ }^{14}$ for $\mathrm{Ni}(100)$ and $\mathrm{Ni}(111)$ using generalized gradient approximation - density functional theory (GGA-DFT) electronic structure theory but the apparent threshold energy for reaction was treated as a free variable. In this way, only 3 parameters are required for d-PMMT calculations, $\left\{E_{0}, \eta_{v}, s\right\}$, where $E_{0}$ is the apparent threshold energy for dissociative chemisorption (see Fig. 4.2), $\eta_{v}$ is the efficacy of vibrational energy relative to translation to provide energy to surmount the barrier to dissociation, and $s$ is the number of surface oscillators. These parameters were fixed by minimizing the average relative discrepancy, ARD, between simulated theoretical and experimental dissociative sticking coefficients,

$$
A R D=\left\langle\frac{\left|S_{\text {theory }}-S_{\text {expt }}\right|}{\min \left(S_{\text {theory }}, S_{\text {exp } t}\right)}\right\rangle
$$

for a limited set of nonequilibrium supersonic molecular beam experiments for each metal single crystal surface. Once the model parameters were defined it was possible to simulate dissociative sticking coefficients for any experiment for which the initial energy distributions are known. For supersonic molecular beam experiments involving a thermal nozzle at temperature, $T_{N}$, it was assumed that the vibrational temperature of the beam molecules was fixed by the nozzle temperature, $T_{v}=T_{N}$, and that rotational cooling in the supersonic expansion left the beam molecules with a rotational temperature of $T_{r}=0.1 T_{N}$. 


\subsection{Results and Discussion}

\subsection{A. $\operatorname{Ru}(0001)$}

The optimal d-PMMT parameter set, determined by minimization of the ARD to the $\mathrm{CH}_{4} / \mathrm{Ru}(0001)$ data shown in Fig. $4.3 \mathrm{~b}$, is $\left\{E_{0}=65.6 \mathrm{~kJ} / \mathrm{mol}, s=1, \eta_{v}=0.50\right\}$ with an overall average relative discrepancy (ARD) between the d-PMMT and experiment equal of $185 \%$. An alternative parameter set, optimized for the data shown in Fig. $4.3 \mathrm{c}$ was found to be $\left\{E_{0}=57.9 \mathrm{~kJ} / \mathrm{mol}, s=1, \eta_{v}=0.50\right\}$. Use of this parameter set overpredicted all of the remaining dissociative sticking coefficients shown in Figure 4.3 with an overall ARD between theory and experiment of $463 \%$, as well as giving much poorer agreement with the time-of-flight experiments in Fig 4.4b. Consequently, the parameter set derived from optimization to the Fig. 4.3b data was used for all of the $\mathrm{Ru}(0001)$ simulations. This optimal parameter set for methane dissociation on $\mathrm{Ru}(0001)$ is similar to the optimal parameters determined for methane dissociation on $\operatorname{Pt}(111)\left\{E_{0}=57.9 \mathrm{~kJ} / \mathrm{mol}, s=2, \eta_{v}=0.40\right\} .^{96} \operatorname{Pt}(111)$ and $\operatorname{Ru}(0001)$ atoms have similar atomic radii (c.f., $1.33 \AA$ and $1.34 \AA$ respectively), similar surface atom densities (c.f. $1.30 \times 10^{15}$ atoms $/ \mathrm{cm}^{2}$ and $1.42 \times 10^{15}$ atoms $/ \mathrm{cm}^{2}$ respectively), hexagonal surface atom arrangements, although Pt exhibits fcc and Ru exhitbits hcp packing, but the surfaces have significantly different mean phonon frequencies (i.e., $v_{s}=\left(\frac{3}{4}\right) k_{B} T_{\text {Debye }} / h=120$ and $310 \mathrm{~cm}^{-1}$, respectively) and Debye temperatures (i.e., $T_{D e b}=234$ and $600 \mathrm{~K}$, respectively). Pt atoms are almost twice as massive as Ru (c.f. $195 \mathrm{amu}$ versus $101 \mathrm{amu}$ ) and the resulting lower mean phonon frequency may facilitate gas-surface energy mixing and thereby account for the difference in the number of surface oscillators $s$. On the other hand, the $\mathrm{CH}_{4} / \mathrm{Pt}(111)$ transition state occurs across two platinum atoms at a bridge site whereas the $\mathrm{CH}_{4} / \mathrm{Ru}(0001)$ transition state occurs over only one ruthenium atom at a top site according to DFT calculations. Consequently, 
the surface oscillator number, s, may correlate with the number of surface atoms involved in the reactive transition state.

The d-PMMT simulations for methane dissociative sticking on ruthenium are shown in Fig. 4.3. Mortensen et al.'s experimental sticking coefficients ${ }^{35}$ for two isotopomers of methane, $\mathrm{CH}_{4}$ and $\mathrm{CD}_{4}$, are shown in Fig. 4.3(a) along with the corresponding d-PMMT predictions (ARD $=37 \%$ ). $\mathrm{d}$-PMMT is able to reproduce the order of magnitude of the sticking coefficients, although it predicts a shallower slope than experimentally observed, particularly for $\mathrm{CD}_{4}$. The slope of the sticking coefficients is primarily determined by the entropy of the system which is dependent on the vibrational frequencies and degeneracies of the transition state. ${ }^{97,}{ }^{98}$ The steepness of the experimental $\log \left[S\left(E_{\mathrm{n}}\right)\right]$ slope relative to the d-PMMT predictions suggests a "looser" reactive transition state. Mortensen et al. speculate the large observed kinetic isotope effect (reported as $\sim 20$ ) is due to classical effects, including the zero point energy correction of the reaction threshold energy and frequency shifts for the incident molecule and molecule-surface vibrations. This is not the case according the d-PMMT model used here, which includes all these effects as well as tunneling to arrive at a KIE of 4.5 for the experimental conditions of Fig. 4.3(a). Luntz and coworkers have also measured supersonic molecular beam dissociative sticking coefficients as a function of inverse surface temperature at fixed $E_{n}$ and $T_{n}$ as shown in Fig. 4.3(b) ${ }^{35}$ Here, d-PMMT dissociative sticking coefficients reproduce the experimental data with an $\mathrm{ARD}=28 \%$.

Dissociative sticking coefficients obtained by Larsen et al. ${ }^{34}$ using a different supersonic molecular beam apparatus are plotted in Fig. 4.3(c). The d-PMMT model underestimates the sticking for all seed mixtures to yield an ARD of $345 \%$ for Fig 4.3(c). The Luntz ${ }^{8}$ and the Larsen molecular beam data are not in good quantitative agreement, but the qualitative variance of the Larsen $S\left(E_{n}\right)$ with $T_{N}$ seems adequately replicated by the d-PMMT model. 
Fig. 4.3(d) compares sticking coefficients determined from thermal equilibrium bulb experiments, ${ }^{32,} 33$ with d-PMMT calculations. The d-PMMT underpredicts the equilibrium experimental data, with an ARD of $237 \%$, but mimics the curvature of the experimental data. In any event, all the dissociative sticking coefficients shown in Fig. 4.3(d) are three to four orders of magnitude higher than those based on nanocatalyst turnover rates from thermal catalysis experiments.

In Figure 4.4, PMMT detailed balance product state distributions, $\mathrm{P}\left(\mathrm{E}_{\mathrm{t}}\right)$, for the associative desorption of $\mathrm{CH}_{3(c)}+H_{(c)} \rightarrow \mathrm{CH}_{4(g)}$ from $\mathrm{Ru}(0001)$ are compared to those derived from measured time-of-flight distributions for three different laser pulse conditions corresponding to laser induced thermal reaction at three different most probable reaction temperatures under the $\sim 10^{10} \mathrm{~K} / \mathrm{s}$ laser heating rate. The d-PMMT slightly overpredicts the mean energy of the reactive flux (e.g. $\left\langle E_{t}\right\rangle_{d-P M M T}=51 \mathrm{vs} .\left\langle E_{t}\right\rangle_{\text {LITR }}=48.2 \mathrm{~kJ} / \mathrm{mol}$ at $\mathrm{T}_{\mathrm{s}}=875 \mathrm{~K}$ ) but qualitatively reproduces the shape of the distributions.

Figure 4.5a compares d-PMMT thermal dissociative sticking coefficients, $S\left(E_{n}, T\right)$, for methane on $\mathrm{Ru}(0001)$ to those derived from the time of flight experiments of Fig. $4.4 \mathrm{~b}$ as a function of normal translational energy calculated according to,

$$
S\left(E_{t}, \vartheta=0^{\circ} ; T\right) \propto \frac{P\left(E_{t}, \vartheta=0^{\circ} ; T\right)}{f_{M B}\left(E_{t}, T\right)}
$$

where $f_{M B}\left(E_{t}, T\right) \propto E_{t} \exp \left(-E_{t} / k_{b} T\right)$ is the flux weighted Maxwell-Boltzmann distribution for molecular translational energy at temperature $T$. The relative dissociative sticking curves derived in this manner have been normalized such that either the normal translational energy integrated sticking, $S\left(\vartheta=0^{\circ} ; T\right)=\int S\left(E_{n}, \vartheta=0^{\circ} ; T\right) f_{M B}\left(E_{n}\right) d E_{n}$, across all translational 
energies is equivalent to d-PMMT predictions, or $S\left(E_{t}=150 \mathrm{~kJ} / \mathrm{mol}, \vartheta=0^{\circ} ; T\right) \rightarrow 1$. Alternatively, if angle-resolved effusive molecular beam dissociative sticking coefficients, $S\left(\vartheta=0^{\circ} ; T\right)=S_{n}(T)$, were available experimentally then absolute $S_{n}\left(E_{n} ; T\right)$ could be derived from the LITR TOF spectra, subject to experimentally $S_{n}(T)$ such that absolute $S_{n}\left(E_{n} ; T\right)$ values could be obtained from experiments aloe, without any input from theoretical modeling.

Calculating the slopes with respect to temperature of the dissociative sticking coefficients $S_{n}\left(E_{n} ; T\right)$ shown in Fig $4.5 \mathrm{a}$ at particular values of $\mathrm{E}_{\mathrm{t}}$ yield activation energies $E_{a}(T)=-k_{B} \partial \ln S / \partial T^{-1}$, which are plotted as a function of normal translational energy in Figure $4.5 \mathrm{~b}$ for both sticking normalized to d-PMMT simulations (solid points) or normalized to 1 at high normal translational energies (exes). The d-PMMT $E_{a}(T)$ initially decreases linearly with a slope of $\partial E_{a}(T) / \partial E_{n}=-1.2$, with the slope beginning to decrease towards 0 as the normal translational energy nears the barrier height to dissociation, $E_{o}$. The s-PMMT model yields similar results but the initial slope is $\partial E_{a}(T) / \partial E_{n}=-1$ near $E_{0}$. Experimental values for the slope are -1.1 when the $\mathrm{E}_{\mathrm{n}}$ integrated $S_{n}\left(E_{n} ; T\right)$ was normalized to d-PMMT simulations of $S_{n}(T)$ and -0.9 when normalized assuming $S\left(E_{n}=150 \mathrm{~kJ} / \mathrm{mol} ; T\right) \rightarrow 1$. For the activated dissociative chemisorption of $\mathrm{H}_{2}$ on $\mathrm{Cu}(111)$, it has been shown experimentally ${ }^{99}$ that for eigenstate-resolved dissociative sticking, $\partial " E_{a}(T) " / \partial E_{n} \approx-1$. A s-PMMT model analytically recovered this result. For polyatomic gas/surface reactivity, the analysis presented here is the first time that experimental data have been shown to yield a similar finding that $\partial E_{a}(T) / \partial E_{n} \approx-1$. The d-PMMT replicates this experimental result. While deviation of the experimental $\partial E_{a}(T) / \partial E_{n}$ from -1 indicates dynamical effects impact the reactivity, it is difficult to directly 
ascribe which degrees of freedom are dynamically biased based on the experimental data alone. Briefly, for thermal equilibrium to determine activation energies of a Tolman ${ }^{38} 100$ form,

$$
\begin{aligned}
E_{a}(T) & =-k_{B} \partial \ln S(T) / \partial T^{-1}=k_{B} T^{2} \partial \ln S(T) / \partial T=\langle E\rangle_{R}-\langle E\rangle \\
& =\sum_{j=r, v, s, t}\left(\left\langle E_{j}(T)\right\rangle_{R}-\left\langle E_{j}(T)\right\rangle\right)
\end{aligned}
$$

where $\left\langle E_{j}\right\rangle_{R}$ and $\left\langle E_{j}\right\rangle$ are the mean energies derived from the $j^{\text {th }}$ degrees of freedom for the successfully reacting gas-surface collision complexes. The temperature dependent experimental sticking is formed as the average microcanonical sticking coefficient over the probability distribution of forming a precursor complex with exchangeable energy, $E^{*}$, for the particular experimental conditions at hand,

$S(T)=\int_{0}^{E^{*}} S\left(E^{*}\right) f\left(E^{*} ; T\right) d E^{*}$

Differentiation of the above with respect to Eq. 4.8 yields the thermal activation energy.

$$
\begin{array}{r}
E_{a}(T)=\left(\frac{\left\langle E_{r}(T)\right\rangle_{R}-\left\langle E_{r}(T)\right\rangle}{\eta_{r}}\right)+\left(\frac{\left\langle E_{v}(T)\right\rangle_{R}-\left\langle E_{v}(T)\right\rangle}{\eta_{v}}\right)+ \\
\left(\frac{\left\langle E_{s}(T)\right\rangle_{R}-\left\langle E_{s}(T)\right\rangle}{\eta_{s}}\right)+\left(\left\langle E_{t}(T)\right\rangle_{R}-\left\langle E_{t}(T)\right\rangle\right)
\end{array}
$$

For spectator degrees of freedom (i.e., rotation for methane dissociative chemisorption on the metals studied in this work) the mean energy of the successfully reacting PCs is equal to that of all PCs initially formed, $\left\langle E_{r}(T)\right\rangle_{R}=\left\langle E_{r}(T)\right\rangle$. As a result, the contribution of spectator degrees of freedom to activation energies calculated using Eq. (4.10) becomes zero.

Unlike in the case for $\mathrm{H}_{2} / \mathrm{Cu}(111)$, where eigenstate resolved associative desorption measurements have been made $^{99}$ allowing for Arrhenius plots to be made with specific rovibrational states, no such data is available for $\mathrm{CH}_{4} / \mathrm{Ru}(0001)$. Instead, the effective activation energies shown here are averaged over the thermal distribution of rotational (n.b., only taken into 
consideration for those cases when rotation is an active degree of freedom), vibrational, and surface states. It is also important to note that these equations are derived from classical statistical mechanics, in the case where tunneling is implemented into the microcanonical sticking coefficient, the effective activation energies may deviate from the expected values as the relative percentage of reactive from tunneling processes increases.

\subsection{B. $\mathrm{Ni}(111)$}

Figure 4.6 compares the dissociative sticking coefficients measured in supersonic molecular beam experiments ${ }^{101}$ over a range of experimental nozzle temperatures and translational energies with the values simulated using the d-PMMT model. The parameters for $\mathrm{Ni}(111)$ data from two different laboratories were determined by minimizing the ARD to the supersonic molecular beam, $S\left(E_{n} ; T_{N}, T_{s}=475 K\right)$ experimental data shown in Fig. 4.6. In consequence, the single $\eta_{v}$ parameter of the d-PMMT model was fixed by a thermal averaging over the likely mode-specific ${ }^{101} \eta_{v}$ s of the active vibrational modes at these four $T_{N}$ values (393, 450, 550, and $1050 \mathrm{~K}$ ) of the Fig. 4.6 data. d-PMMT model parameters of $\left\{E_{0}=76.5 \mathrm{~kJ} / \mathrm{mol}, s=1, \eta_{v}=0.70\right\}$ are obtained. The Chorkendorff and Utz experiments of Figure 4.6 have an overall ARD of $134 \%$, however the points with a nozzle temperature of $450 \mathrm{~K}$ are somewhat anomalous when compared to the other data sets as they are consistently lower than those points taken with a lower nozzle temperature of $393 \mathrm{~K}$. Discounting the $T_{N}=450 \mathrm{~K}$ points, the ARD for Fig. 4.6 drops to $69 \%$.

Supersonic molecular beam sticking coefficients taken with varying nozzle temperatures, normal translational energies, and seed mixtures measured by Lee et al. ${ }^{77}$ in the Ceyer laboratory are plotted in Fig. 4.7(a) and compared to d-PMMT calculations. The d-PMMT model predicts the Fig. 4.7(a) sticking with an overall ARD of 38\%. d-PMMT calculations of dissociative sticking coefficients for methane isotopomers are compared to experimental values for a range of 
nozzle temperatures, $300 K \leq T_{N} \leq 700 K$, in Figure 4.7(b). The d-PMMT kinetic isotope effect, $\mathrm{KIE}=7.59$, is in close agreement to the experimental value of $\mathrm{KIE}=8.53$ over the range of $65 \mathrm{~kJ} / \mathrm{mol} \leq E_{N} \leq 75 \mathrm{~kJ} / \mathrm{mol}$.

Fig. 4.8(a) compares dissociative sticking coefficients determined from thermal equilibrium bulb experiments with d-PMMT calculations. The d-PMMT dramatically underpredicts the equilibrium experimental data, with an ARD of $802 \%$. Additionally, the dPMMT activation energy of $E_{a}=89 \mathrm{~kJ} / \mathrm{mol}$ is high relative to experiment. Fig. 4.8(b) shows d-PMMT predicted dissociative sticking coefficients, $S_{n}\left(T_{g}, T_{s}\right)$, for a normally incident effusive beams with $\mathrm{T}_{\mathrm{s}}$ varying from $300 \mathrm{~K}$ to $1000 \mathrm{~K}$ in increments of $100 \mathrm{~K}$.

Experimental measurements taken by Utz and $\mathrm{Beck}^{101}$ of $\mathrm{CH}_{4} / \mathrm{Ni}(111)$ dissociative sticking coefficients for a thermal nozzle, $v_{3}, 2 v_{3}$, and $3 v_{4}$ state-resolved supersonic molecular beams are presented in Figure 4.9 along with d-PMMT theoretical simulations of the experiments. The d-PMMT model reproduces the experimental measurements surprisingly well given each mode has a different vibrational efficacy as opposed to the single thermally averaged vibrational efficacy used by the d-PMMT model (i.e., $A R D=182 \%$ for Fig. 4.9). At normal translational energies less than $50 \mathrm{~kJ} / \mathrm{mol}$, d-PMMT predictions decrease at a dramatically greater rate with $E_{n}$ than the measured dissociative sticking coefficients. The d-PMMT qualitatively reproduces the $\mathrm{Ni}(111)$ experimental data shown in Figs. 4.6 through 4.9 with an overall ARD of $177 \%$.

Table 4.1 lists experimentally determined eigenstate specific vibrational efficacies and the d-PMMT thermally averaged vibrational efficacy for experimental data. The mode-specific vibrational efficacy, $\eta_{v i b}$, relative to $E_{n}$ was measured experimentally according to, 


$$
\left.\eta_{v i b} \equiv \frac{\Delta E_{n}}{\Delta E_{v}}\right|_{\Delta S_{v}}
$$

where $\Delta S_{v}$ is the change in sticking coefficient in going from ground state molecules in the supersonic beam to vibrationally excited molecules at some particular $E_{n}, \Delta E_{v}$ is the vibrational energy difference between the ground state and vibrationally excited molecules in the beams, and $\Delta E_{n}$ is the change in $E_{n}$ required to gain the same $\Delta S_{v}$ change along the dissociative sticking coefficient curve of the ground state molecules. Given that $\eta_{v i b}$ values for methane dissociative chemisorption on $\mathrm{Ni}(111)$ are vibrational mode-specific, the d-PMMT model provides at best a vibrationally averaged description of the experiments. Disturbingly, the $\mathrm{T}_{\mathrm{N}}=373 \mathrm{~K}$ experimental sticking coefficients appear to deviate substantially from the other heated nozzle supersonic molecular beam data, which the d-PMMT simulates fairly well in Fig. 4.6.

\subsection{C. $\mathrm{Ni}(100)$}

Supersonic molecular beam sticking coefficients as a function of normal translational energy over a range of nozzle temperatures obtained by Holmblad et $\mathrm{al}^{28}$ are plotted in Fig. 4.10(a) with an ARD of $29.4 \%$. The optimal d-PMMT parameter set, determined by minimization of the $\mathrm{ARD}$ to the $\mathrm{CH}_{4} / \mathrm{Ni}(001)$ data shown in Fig. 4.10(a), was $\left\{E_{0}=67.5 \mathrm{~kJ} / \mathrm{mol}, s=1, \eta_{v}=0.72\right\}$. Points created by interpolation (filled) or extrapolations (open) of slices through smooth empirical lines drawn by Holmblad through their Fig. 4.10(a) data at specific translational energies are reproduced in Figure 4.10(b) and compared to d-PMMT $S\left(E_{n}, T_{N}\right)$ simulations.

Holmblad and coworkers measured supersonic molecular beam dissociative sticking coefficients as a function of inverse surface temperature at three combinations of fixed $E_{n}$ and $T_{n}$ 
as shown in Fig. 4.11(a). Here, d-PMMT dissociative sticking coefficients reproduce the experimental data with an ARD $=19.4 \%$. Dissociative sticking coefficients for methane isotopomers on $\mathrm{Ni}(100)$ for a range of nozzle temperatures, $550 \mathrm{~K} \leq T_{N} \leq 1050 \mathrm{~K}$ are shown in Figure 4.11(b) and compared to d-PMMT simulations. The d-PMMT model qualitatively reproduce the experimental data with an ARD of $26.3 \%$ for $\mathrm{CH}_{4}$ and $17.2 \%$ for $\mathrm{CD}_{4}$. The dPMMT kinetic isotope effect, $\mathrm{KIE}=6.38$, was found to be nearly identical to that determined experimentally, $\mathrm{KIE}=6.44$, over the $\mathrm{E}_{\mathrm{n}}$ range explored (i.e. $50 \mathrm{~kJ} / \mathrm{mol} \leq E_{N} \leq 80 \mathrm{~kJ} / \mathrm{mol}$ ). Overall, the d-PMMT model replicates the supersonic beam data taken by Holmblad in Fig 4.10 and 4.11 fairly well, with an ARD of $24.9 \%$.

Figure 4.12 compares angle-integrated, ambient gas dissociative sticking coefficients, $S\left(T_{g}, T_{s}\right)$, measured in thermal bulb experiments by Nielsen et al. ${ }^{19}$ with d-PMMT simulations. The d-PMMT model underpredicts the thermal bulb data by an order of magnitude and achieving an ARD of only $773 \%$. To investigate if rotational degrees of freedom may be efficacious in promoting reactivity, thermal bulb data was compared to d-PMMT calculations with active rotations. As with spectator rotations, the $\left\{E_{0}=55.2 \mathrm{~kJ} / \mathrm{mol}, s=1, \eta_{v}=0.68\right\}$ parameters of the d-PMMT model with active rotation were optimized to the Holmblad supersonic molecular beam data of Fig.4.10(a) and provided an ARD for the data in Figs. 4.10 and 4.11 of $28 \%$. The dPMMT model with active rotations overpredicts the thermal bulb data and yields an ARD of $658 \%$, comparable to that from d-PMMT calculations with spectator rotations. Consequently, there seems to be no advantage to including rotation as an active degree of freedom in $\mathrm{CH}_{4} / \mathrm{Ni}(100)$ dissociative chemisorption.

Figure 4.13 compares $\mathrm{CH}_{4} / \mathrm{Ni}(100)$ dissociative sticking coefficient derived from thermal nozzle and $v_{1}, v_{3}$, and $2 v_{3}$ state-resolved supersonic molecular beam experiments with d- 
PMMT simulations. The d-PMMT model overpredicts the experimental measurements with an overall ARD for Figure 4.13 of $482 \%$. Scaling the latest experimental thermal nozzle data by 7 would bring it into registry with the Chorkendorff experiments, on which the d-PMMT parameters were fixed, and thereby reduce the ARD between d-PMMT simulations and Fig. 4.13 experiments to $84.7 \%$. Similar to the $\mathrm{CH}_{4} / \mathrm{Ni}(111)$ case, the d-PMMT simulations have a similar curvature at normal translational energies above $50 \mathrm{~kJ} / \mathrm{mol}$, but show greater change in sticking with normal translation at lower energies. The overall average relative discrepancy (ARD) between the d-PMMT and the Ni(111) experiments in Figures 4.10 through 4.13 is $190 \%$.

Figure 4.14 shows d-PMMT simulations of $S_{n}\left(T_{g}, T_{s}\right)$ dissociative sticking coefficients for methane on $\mathrm{Ni}(100)$ for a normally incident effusive beam under non-equilibrium gas-surface conditions. Reactivity is predicted to be strongly dependent on gas temperature, but shows little change in reactivity with surface temperature under hyperthermal gas conditions (i.e., $T_{g} \gg T_{s}$ ).

The slopes of the relative dissociative sticking coefficients measured by Holmblad et al. in Fig 4.11(a) and 4.10(b) yield effective activation energies $" E_{a}\left(T_{j}\right) "=-k_{B} \partial \ln S / \partial T_{j}^{-1}=\left(\left\langle E_{j}\right\rangle_{R}-\left\langle E_{j}\right\rangle\right) / \eta_{j}$, for the surface and vibrational degrees of freedom, respectively, which are plotted as a function of normal translational energy in Figure 4.15(a) and (b). The d-PMMT " $E_{a}\left(T_{s}\right)$ " values match those calculated from the experimental data of 4.11(a) with good fidelity. At the elevated nozzle temperatures of the experiments, there is little dependence on the " $E_{a}\left(T_{s}\right)$ " with normal translational energy. For example at $\mathrm{T}_{\mathrm{N}}=850 \mathrm{~K}$ the experimental $\partial^{\prime \prime} E_{a}\left(T_{s}\right) " / \partial E_{n}=-0.07$ compares to the d-PMMT value at $E_{n}=40 \mathrm{~kJ} / \mathrm{mol}$ of $\partial " E_{a}\left(T_{s}\right) " / \partial E_{n}=-0.04$. At the lower temperatures of $\mathrm{T}_{\mathrm{N}}=600 \mathrm{~K}$ and 300 $\mathrm{K}$, the d-PMMT predicts $\partial " E_{a}\left(T_{s}\right) " / \partial E_{n}=-0.18$ and -1.03 , respectively. 
In contrast, the plot of " $E_{a}\left(T_{N}\right)$ "vs. $E_{\mathrm{n}}$ shown in Figure 4.15(b) shows a strong dependence on $\mathrm{E}_{\mathrm{n}}$ at elevated nozzle temperatures (e.g., " $E_{a}\left(T_{N}\right)$ " near $\mathrm{T}_{\mathrm{N}}=850 \mathrm{~K}$ increases from $31 \mathrm{~kJ} / \mathrm{mol}$ to $75 \mathrm{~kJ} / \mathrm{mol}$ as $\mathrm{E}_{\mathrm{n}}$ decreases from 60 to $20 \mathrm{~kJ} / \mathrm{mol}$ ). Effective activation energies derived from varying the nozzle temperature are equivalent to those of varying vibrational temperature (i.e. " $E_{a}\left(T_{N}\right) "=" E_{a}\left(T_{v}\right) "$ ) because molecular rotations are frozen out by the supersonic expansion and the incident molecules' vibrational temperature is presumed to be given by the nozzle temperature (i.e., $T_{r}=0.1 T_{N}$ and $T_{v}=T_{N}$ ). d-PMMT predictions of $" E_{a}\left(T_{N}\right)$ "qualitatively reproduce those calculated from interpolation of the Fig. 4.10(a) experimental data, but overpredict those points taken from extrapolation.

Under similar experimental conditions, the " $E_{a}\left(T_{j}\right)$ "s for $\mathrm{CH}_{4} / \mathrm{Pt}(111)$ and $\mathrm{Ni}(100)$ are shown in Figure 4.16(a and b) and (c and d). For the case when $T_{N} \gg T_{s}$, Fig. 4.16(a and b), on both single crystal metal surfaces energy from vibration is predicted to play the dominant role in supplying reactivity. In contrast, when $T_{N} \ll T_{s}$, energy from the surface plays a more dominant role than vibration in supplying the energy needs to react. Instead of one degree of freedom being the predominant means of supplying the energy required to surmount the activation barrier under all reactive conditions, it is instead the availability of exchangeable energy under a particular set of experimental conditions that will govern how different degrees of freedom contribute to reactivity. Under experimental conditions where the availability of energy from the incident gas molecules is relatively modest, the surface will always be the dominant provider of the energy necessary to react, and vice versa. The efficacy of vibration dynamical constraint effectively reduces the vibrational temperature of the molecular vibrational heat reservoir. For $\operatorname{Pt}(111)$, the significantly smaller efficacy of vibration relative to that of $\mathrm{Ni}(100), \eta_{v}=0.40$ vs. 0.72 , severely curtails the availability of vibrational energy for reaction. 


\subsection{D. $\operatorname{Ir}(111)$}

Fig. 4.17 shows dissociative sticking coefficients obtained by the molecular beam experiments of Seets' et al. ${ }^{10}$ along with d-PMMT simulations using the optimized parameter set $\left\{E_{0}=48.2 \mathrm{~kJ} / \mathrm{mol}, s=1, \eta_{v}=0.05\right\}$. The d-PMMT parameters were optimized using the Fig. 4.17 experimental data for molecular beams of methane seeded in either $\mathrm{He}$ or $\mathrm{H}_{2}$ (i.e., $E_{n} \gtrsim 10$ $\mathrm{kJ} / \mathrm{mol}$ ) alone. ${ }^{10}$ The ARD between theory and experiment for the Fig. 4.17(a) $S\left(E_{n}, T_{s}=1000 \mathrm{~K}\right)$ was $124 \%$. The dependence of the dissociative sticking on surface temperature at fixed normal translational energies shown in Fig 4.17(b) was replicated with an ARD of $22 \%$.

While the d-PMMT captures the curvature of the dissociative sticking curve of 4.17(a), it fails to quantitatively predict the sharply varying behavior at low translational energies, $E_{n}<10 \mathrm{~kJ} / \mathrm{mol}$. The boomerang behavior at low $\mathrm{E}_{\mathrm{n}}$ has been discussed elsewhere ${ }^{102}$ using a statistical (s-) PMMT model and is beyond the scope of this chapter.

Fig. 4.18 shows thermal sticking under both equilibrium and non equilibrium conditions for both $\mathrm{CH}_{4}$ and $\mathrm{CD}_{4}$. Thermal equilibrium data for $\mathrm{CH}_{4}$ determined by Wei and Iglesia ${ }^{94}$ and Jachimowski et al. ${ }^{11}$ give $E_{a}=72 \mathrm{~kJ} / \mathrm{mol}$ and $E_{a}=81 \mathrm{~kJ} / \mathrm{mol}$, respectively, significantly higher than the d-PMMT prediction of $E_{a}=66.4 \mathrm{~kJ} / \mathrm{mol}$. The d-PMMT predicted activation energy is lower than the experimental values, differing by $\sim 10 \mathrm{~kJ} / \mathrm{mol}$. The d-PMMT model predicts a reaction threshold energy of $E_{0}=51.1 \mathrm{~kJ} / \mathrm{mol}$, which is between the limits of theoretical values obtained from DFT calculations (i.e. $15 \pm 10 \mathrm{~kJ} / \mathrm{mol}^{95}$ and $76 \mathrm{~kJ} / \mathrm{mol}^{49}$ ). d-PMMT predictions including non-equilibrium effusive beam reactivity is shown in Figure 4.19.

Kinetic isotope effects (KIE) measured by Seets et al. ${ }^{10}(\sim 3.4)$ and Jachimowski et al. ${ }^{11}$ ( 1.9) under nonequilibrium thermal conditions indicate a much smaller kinetic isotope effect than that calculated from d-PMMT simulations of 6.2. However, Seets concedes to 
contamination of the $\mathrm{CD}_{4}$ gas, lowering the KIE he observes. In addition, d-PMMT predictions for the KIE of 6.58 for sticking coefficients obtained under thermal equilibrium conditions are significantly closer to the experimental values $1.70-1.81$ observed by Wei and Iglesia. ${ }^{94}$

\subsection{E. Comparison Between Surfaces}

\subsection{E.1. Catalysis}

Thus far, the d-PMMT has been applied to the dissociation of several alkanes on single crystal transition metal surfaces [e.g., $\mathrm{CH}_{4}$ on $\mathrm{Pt}(111), \mathrm{Ni}(100), \mathrm{Ni}(111), \mathrm{Ru}(0001)$, and $\left.\operatorname{Ir}(111)\right]$. One of the most important features of the d-PMMT is its ability to bridge the nonequilibrium gap between surface science and experiment by making predictions relevant to thermal catalysis. Thermal dissociative sticking coefficients for $\mathrm{CH}_{4}$ on single crystal surfaces and supported nanocatalysts $^{1,3-5}$ are shown in Fig. 4.20. d-PMMT predictions were based on optimization to molecular beam experiments and agree with thermal bulb experiments for these metal single crystal surfaces. d-PMMT calculations predicts that $\operatorname{Ir}(111)$ is the most reactive transition metal with respect to methane dissociation followed by $\operatorname{Pt}(111)$, then $\mathrm{Ru}(0001)$ and $\mathrm{Ni}(100)$, and finally $\mathrm{Ni}(111)$ being the least reactive at all temperatures. Interestingly, under isothermal conditions where gas and surface temperatures are above $400 \mathrm{~K}, \mathrm{Ni}(100)$ reactivity is found to pass that of $\mathrm{Ru}(0001)$. This trend of increasing reactivity continues such that $\mathrm{Ni}(100)$ becomes nearly as reactive in methane dissociative chemisorption as both $\operatorname{Pt}(111)$ and $\operatorname{Ir}(111)$ at $1000 \mathrm{~K}$. Contrastingly, Wei and Iglesia's thermal catalyst experiments indicate that $\operatorname{Pt}(111)$ is the most active surface for methane dissociative chemisorption followed by $\operatorname{Ir}(111), \mathrm{Ni}(100)$ and finally $\operatorname{Ru}(0001) .{ }^{1}$ When plotted on a log scale as done in Fig. 4.20, the difference between catalysts becomes almost indistinguishable, while the d-PMMT predicts the variance in reactivity across the transition metals to be more dramatic.

Additionally, sticking coefficients derived from catalyst turnover rates on various diameter

nanoparticles are three to four orders of magnitude lower than the d-PMMT predictions and 
surface science experiments. This result is counter-intuitive since the preponderance of step edges on the nanoparticles should result in higher thermal sticking coefficients than those observed on very flat single crystal surfaces like $\mathrm{Ru}(0001)$. Structure sensitivity in alkane dissociative sticking coefficients has been shown on single crystal surfaces where sticking coefficients are higher for surfaces with more step edges. For example, dissociative sticking coefficients for $\mathrm{CH}_{4}$ and $\mathrm{C}_{2} \mathrm{H}_{6}$ measured on $\operatorname{Pt}(110)^{103}$ and $\operatorname{Pt}(111)^{39,104}$ were recently compared in Fig. 2 of Ref. 81, revealing one to two orders of magnitude enhancement in sticking for methane dissociation on $\operatorname{Pt}(110)$ versus $\operatorname{Pt}(111)$. The preponderance of step edges, and therefore dangling bonds, on the $\operatorname{Pt}(110)$ surface as compared to the $\mathrm{Pt}(111)$ surface is believed to account for this improvement in sticking coefficients. Similarly, structure sensitivity has been show to increase reactivity on nanocatalysts where reaction turnover rates are higher for surfaces with more dispersion. ${ }^{1}$

Although it is not exactly clear why nanocatalyst sticking coefficients are low with respect to those of single crystal surfaces, it has been speculated that carbon coverage may poison the surface and affect the reactivity. ${ }^{45}$ Two possible explanations can be offered which relate to carbon deposition on the nanoclusters. First, carbon atoms deposited on the nanoparticles may change the overall electronic structure of the surface and reduce the reactivity as compared to the clean transition metal. Alternatively, carbon buildup on the surface may limit the quantity of contiguous metal atoms, restricting the number of reactive ensembles of metal atoms available on each nanoparticle. According to d-PMMT, the minimum number of unoccupied adjacent metal atoms necessary for dissociation is $s$ (i.e., the number of surface oscillators participating in energy exchange within the reactive precursor complexes), but the reaction products will likely need additional sites to ultimately lodge. Repulsive forces between carbon atoms on the surface may increase the number of non-carbon-bonded metal atoms necessary to achieve dissociation. In clean ultrahigh vacuum (UHV) surface science experiments, these effects would not be noticeable under the low carbon coverage conditions investigated. ${ }^{105}$ Wei and Iglesia have accounted for 
transport effects in their experiments and discuss the possibility that nanoclusters undergo nearsurface melting which reduces the number of uncoordinated reactive bonds available for dissociation. ${ }^{3}$

To analyze the contribution different forms of energy make to the overall sticking under thermal equilibrium conditions, fractional energy uptakes, defined as the mean energy for a particular degree of freedom (i.e. translation, vibration, and surface) of the reacting molecules divided by the total mean energy of the species that go on to react, $f_{j}=\left\langle E_{j}\right\rangle_{R} /\left\langle E^{*}\right\rangle_{R}$. Figure 4.21 shows the fractional energy uptakes as a function of temperature for methane dissociative chemisorption on the transition metals studied under thermal conditions. Perhaps a more useful means to compare the influence of the individual degrees of freedom is shown in Figure 21, where the mean energies $(a \& b)$ and fractional energy uptakes (c\&d) are plotted at a specific thermal temperature as a function of single crystal metal surface. As illustrated in Fig. 4.21 and 4.22 , the contribution of surface degrees of freedom are equal to that of normal translation for all surfaces studied in this chapter and were previously found to be the most important in the dissociative chemisorption of methane $\operatorname{Pt}(111)$. In the case of both faces of nickel, vibration plays the predominant role in promoting reacticity at temperatures about $600 \mathrm{~K}$, with the change in importance relative to the other transition metal surfaces shown clearly in Fig. 4.22. At catalytically relevant temperatures, $\mathrm{T}=873 \mathrm{~K}$, gas degrees of freedom contribute approximate $75 \%$ of the energy for successfully reacting PCs for $\mathrm{Ni}(111)$ and $\mathrm{Ni}(100)$, as opposed to $41 \%$, $62 \%$ and $50 \%$ for $\operatorname{Pt}(111), \operatorname{Ru}(0001)$, and $\operatorname{Ir}(111)$ respectively. While gas degrees of freedom are clearly important in promoting reactivity, these results are particularly interesting since most experiments have focused primarily on sticking enhancements from translational and vibrational degrees of freedom, but have largely neglected contributions from the surface, and nearly all theoretical studies hold the surface as a spectator to the reactivity. 
Figure 4.23 relates the zero point corrected barrier heights calculated by Jackson ${ }^{14}$, who seems unique in the literature by calculating properties for a number of $\mathrm{CH}_{4} /$ metal systems using identical basis sets and functionals in a consistent manner, to the threshold barrier height to reaction determined from experiments using the d-PMMT model. The relation shown in Fig. 4.23 is an interesting one because it suggests that the many electron van der Waals interaction between the products of dissociative chemisorption and the surface may account for the difference between recently calculated GGA-DFT and d-PMMT threshold barriers for dissociative chemisorption of the methane. Typical GGA-DFT calculations of reaction energetics do not directly account for van der Waals interactions, ${ }^{106}$ and the addition of correction for van der Waals attraction to DFT calculated reaction energetics is only beginning to be explored in the theoretical literature of methane reactivity at surfaces. ${ }^{107}$ d-PMMT threshold barriers may be useful to provide a benchmark for future DFT functional development including van der Waals interactions, which could be used to predict reactivity of next generation catalysts.

\subsection{F. Polanyi Relationship}

For the surfaces investigated in this and the previous chapters, Figure 4.24(a) relates transition state characteristics from GGA-DFT calculations (i.e. C-H bond length and $\mathrm{C}$ height above the metal surface) to d-PMMT efficacy of vibration, $\eta_{v}$, determined from thermal nozzle supersonic beams. Assuming the Polanyi rules are applicable to direct ${ }^{108}$ dissociative chemisorption gas-surface reactions, for an early barrier (i.e where the transition state is located closer to and has characteristics of the reactant species), $\eta_{v}<1$, whereas for a late barrier $\eta_{v}>1 .^{109}$ The length of the $\mathrm{C}-\mathrm{H}$ bond extension in the transition state and the height of the dissociating methane above the surface were found to be linearly related to the d-PMMT efficacy 
of vibration with positive and negative slope, respectively. The vibrational efficacy tracked the GGA-DFT transition state characteristics for earlier and later transition states in a manner consistent with the Polanyi rules.

\subsection{Summary}

The dissociative chemisorption of methane on a series of transition metal surfaces was studied through the application of a dynamically biased precursor mediated microcanonical trapping (d-PMMT) model with ARDs of $185 \%, 177 \%, 190 \%$, and $156 \%$ for $\operatorname{Ru}(0001), \mathrm{Ni}(111)$, $\mathrm{Ni}(100)$, and $\operatorname{Ir}(111)$, respectively. Apparent threshold energies for dissociation were found to be in general lower than values calculated from electronic structure theory. The value of the dynamical biasing constraint, $\eta_{v}$, on the efficacy of vibrational relative to translational energy to promote reactivity ranged from 0.05 for $\operatorname{Ir}(111)$ to 0.72 for $\mathrm{Ni}(100)$. Correlation between transition state characteristics calculated from GGA-DFT potential energy surfaces and d-PMMT parameters was made. In particular, the value of $\eta_{v}$ was found to be directly related to the length of the dissociating $\mathrm{C}-\mathrm{H}$ bond at the transition state. Rotation was found to be a spectator degree of freedom for all single crystal metals studied. At catalytically relevant temperatures, reactivity for all metals was found to be higher than those calculated from supported nanocrystalites, as well different order of reactivate surfaces, with d-PMMT calculated $S(T=873 \quad K)$ $\operatorname{Ir}(111)>\operatorname{Pt}(111)>N i(100)>R u(0001)>N i(111)$. 

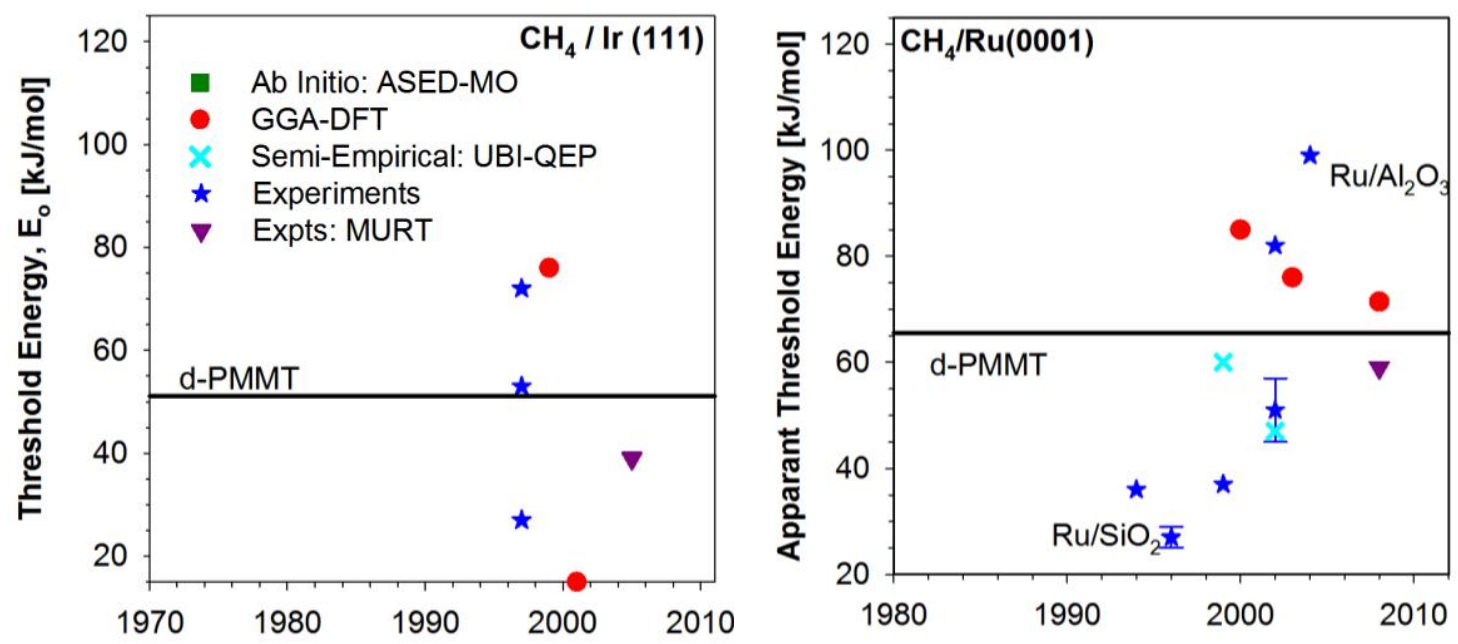

(a)

Publication Year

(b)

Publication Year

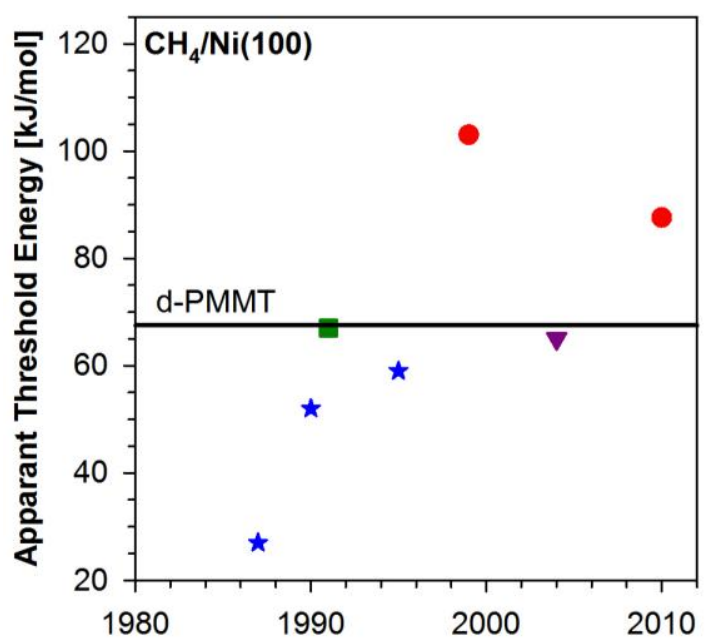

(c)

Publication Year

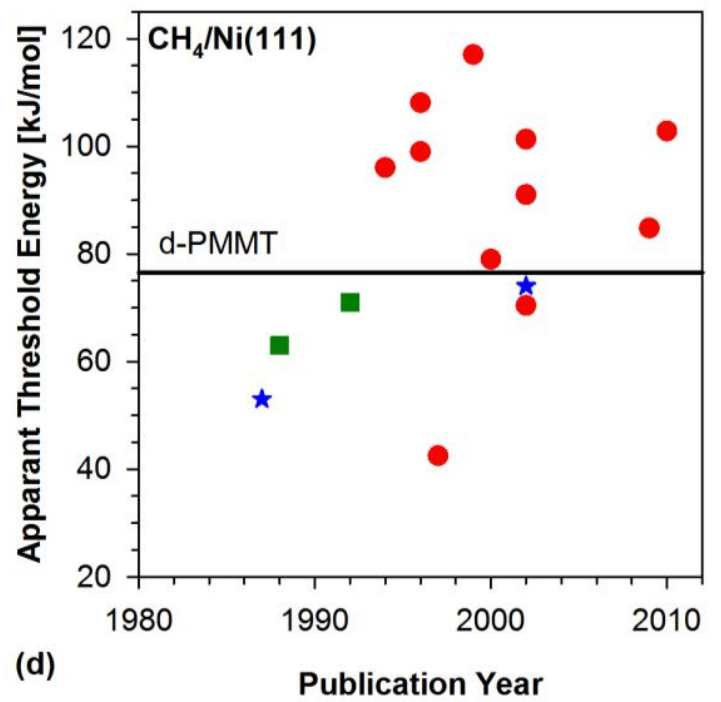

Figure 4.1. Apparent threshold energies for methane dissociative chemisorption on transition metal single crystal surfaces calculated using electronic structure theory techniques ${ }^{14,34,46-61}$ or kinetic modeling of various kinds of experimental data. ${ }^{26,33-45}$ 


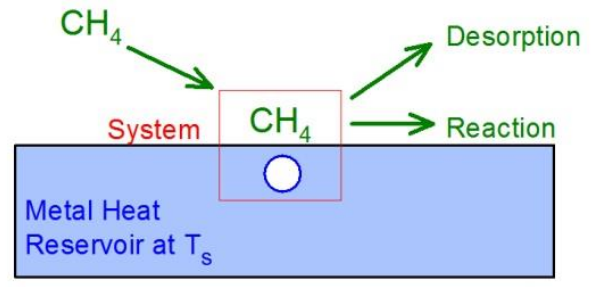

$$
\mathrm{CH}_{4(g)} \stackrel{F\left(E^{*}\right)}{\rightleftarrows} \mathrm{k}_{D} H_{4(p)} \stackrel{k_{R}}{\longrightarrow} \mathrm{CH}_{3(c)}+H_{(c)}
$$

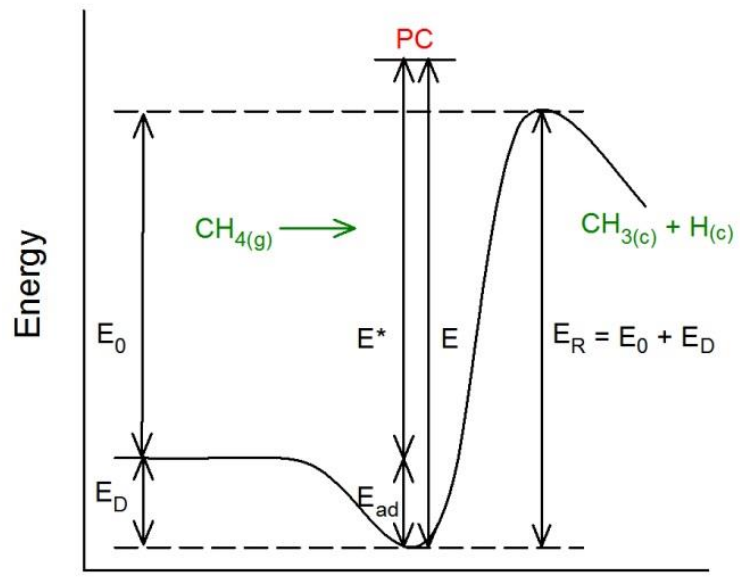

Reaction Coordinate

Figure 4.2. Schematic depiction of the kinetics and energetics of methane dissociative chemisorption via precursor-mediated microcanonical trapping (PMMT). At energies sufficient to react, collisionally formed precursor complexes, PCs, comprised of a methane molecule interacting with $s$ surface oscillators in the spatial vicinity of the physisorption well, are presumed to become transiently trapped between the transition states for desorption and reaction. Zero-point energies are implicitly included within the potential energy curve along the reaction coordinate. See text for further details. 


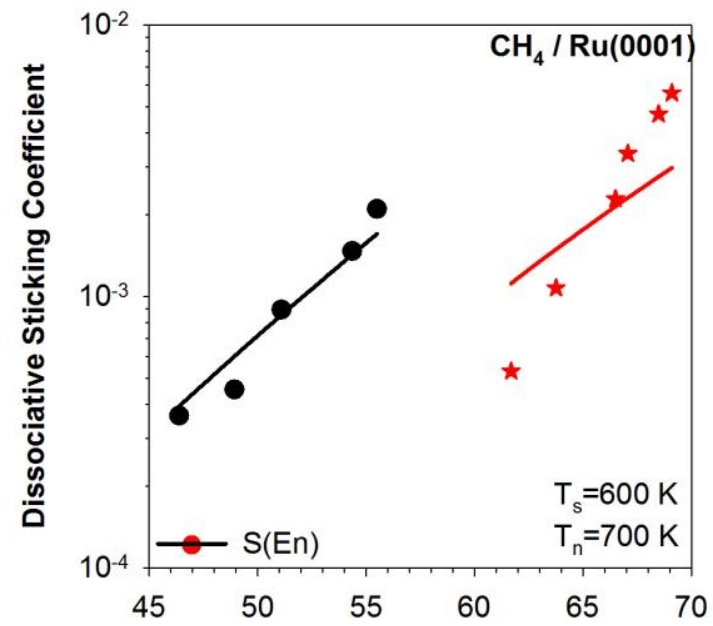

(a) Normal Translational Energy, $E_{n}[\mathrm{~kJ} / \mathrm{mol}]$

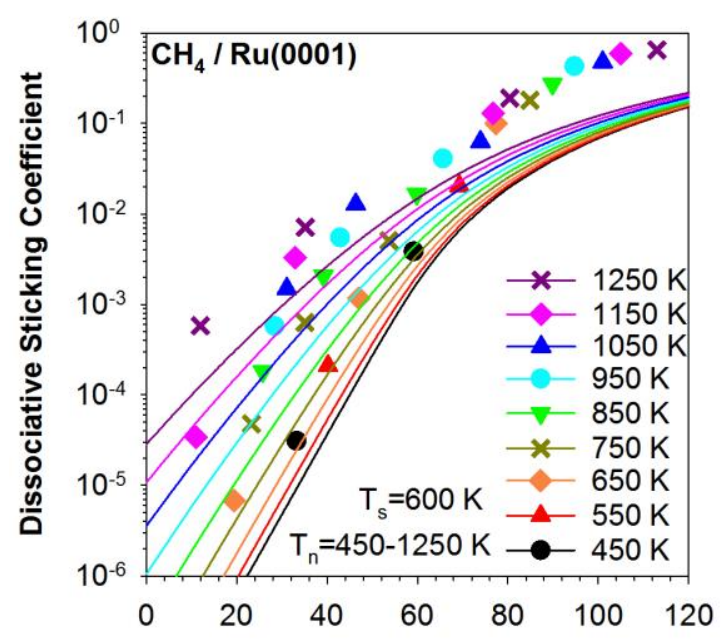

(c)

Normal Translational Energy, $E_{n}[\mathrm{~kJ} / \mathrm{mol}]$
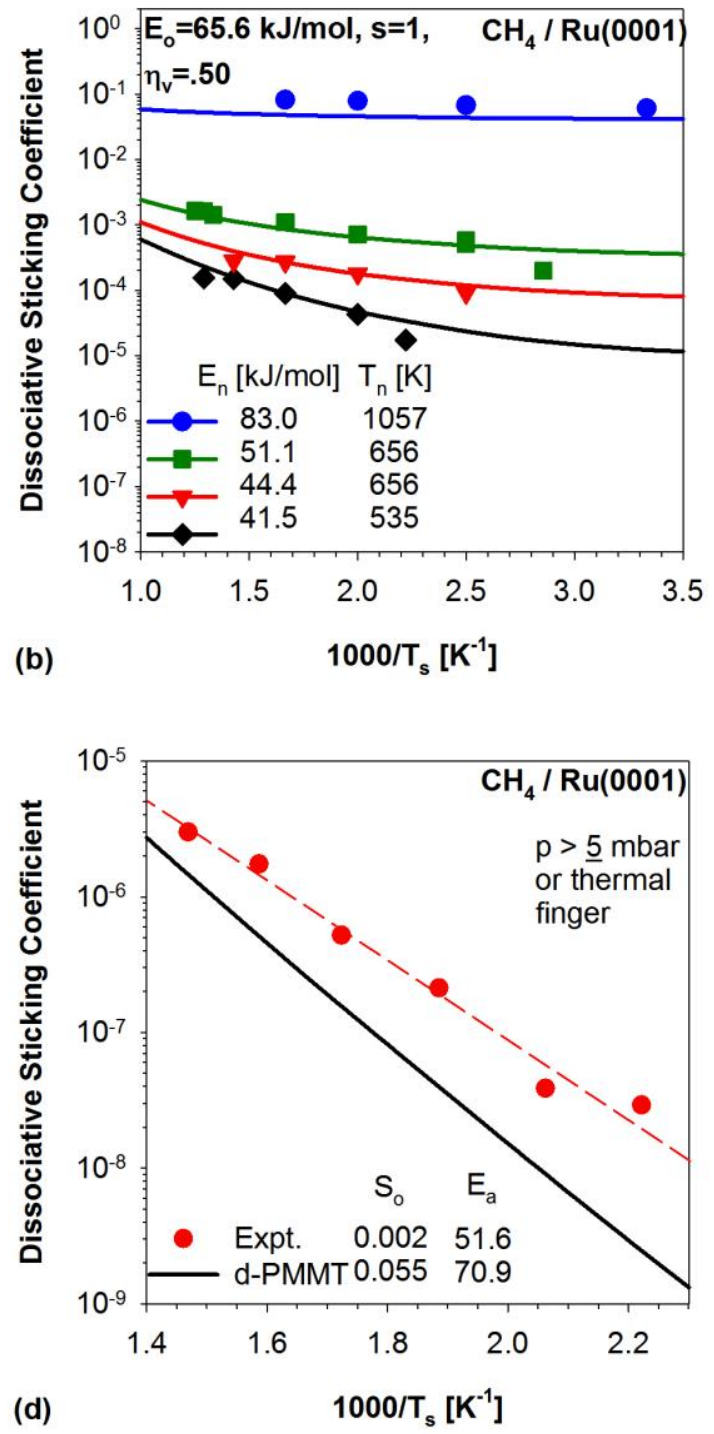

Figure 4.3. Dissociative sticking coefficients for $\mathrm{CH}_{4}$ incident on $\mathrm{Ru}(0001)$. Data from supersonic beam ${ }^{34,35}$ and thermal bulb ${ }^{42}$ experiments (points) are compared to d-PMMT simulations (lines) with rotation acting as a spectator and vibrational efficacy parameter, $\eta_{v}$, with parameters fixed by optimization of simulations to the $T_{s}$-dependent data of (b) alone. 

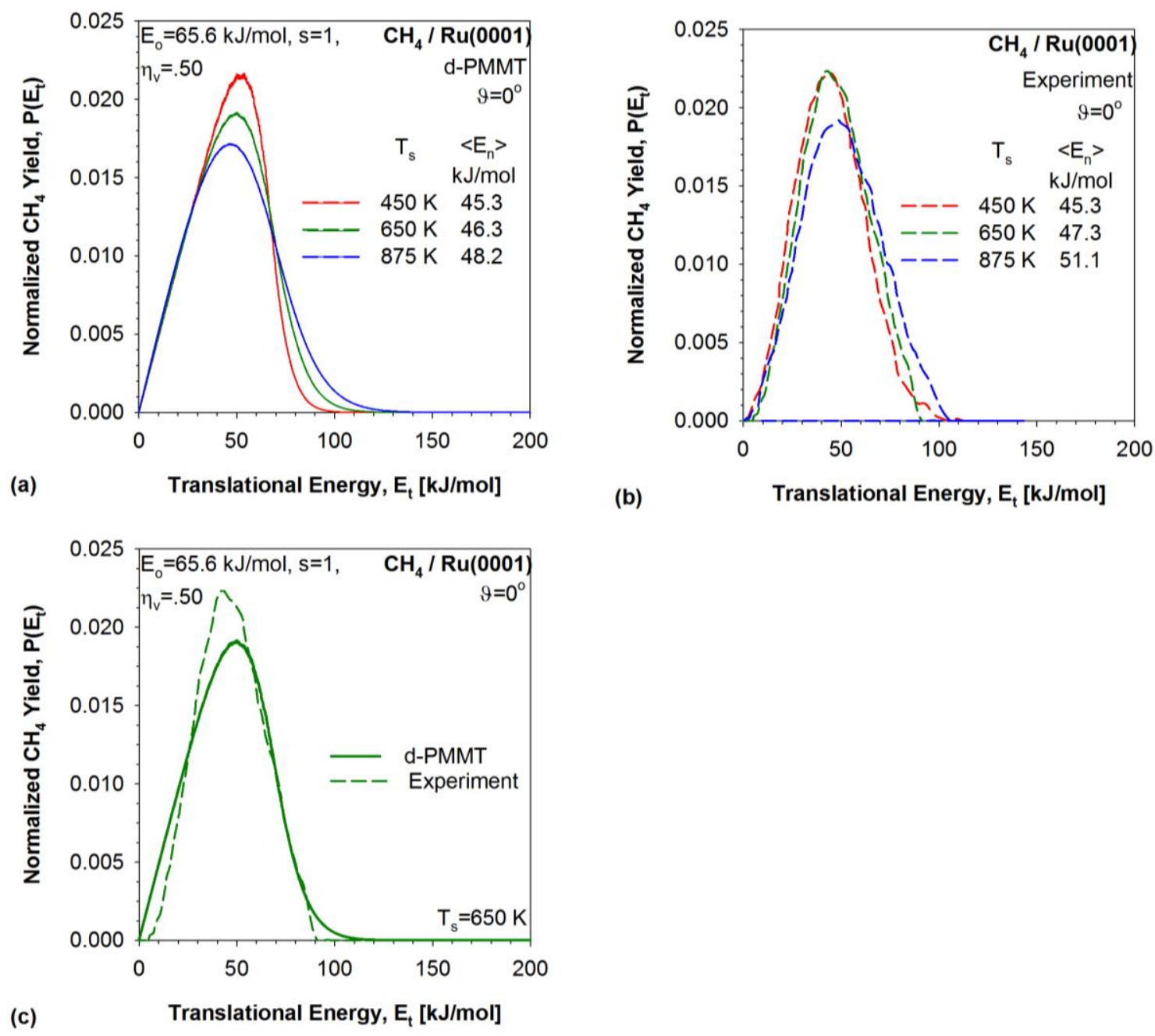

Figure 4.4. (a) d-PMMT simulations (lines) are compared to (b) experimental data ${ }^{35}$ (dashed lines) for the $\mathrm{CH}_{4(\mathrm{~g})}$ product translational energy distributions derived from time-of-flight spectra of the laser induced thermal reaction $\mathrm{CH}_{3(c)}+H_{(c)} \rightarrow \mathrm{CH}_{4(g)}$ on $\mathrm{Ru}(001)$ for different laser pulse conditions inducing thermal reaction at experimentally calibrated surface temperatures of $450 \mathrm{~K}, 650 \mathrm{~K}$, and $875 \mathrm{~K}$. (c) Overlaid comparison of the smoothed, locally averaged theoretical and experimental $\mathrm{CH}_{4(g)}$ product translational energy distribution for the LITR at $\mathrm{T}_{\mathrm{s}}=650 \mathrm{~K}$. 


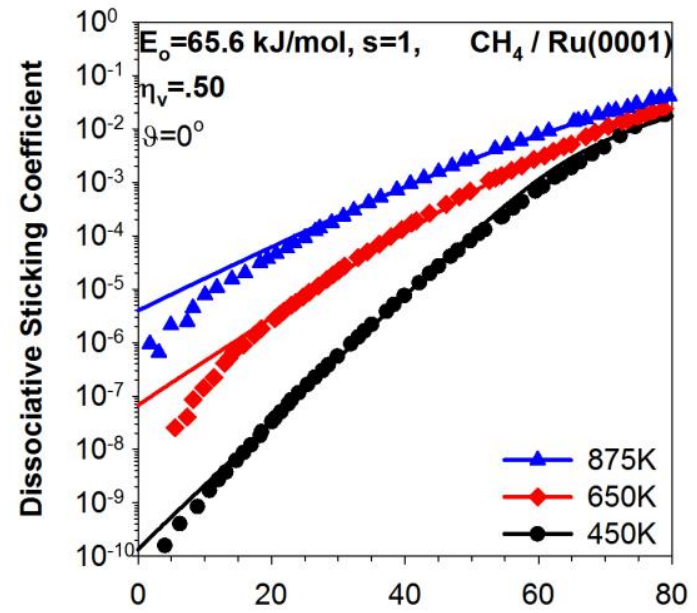

(a)

Normal Translational Energy, $E_{n}[\mathrm{~kJ} / \mathrm{mol}]$

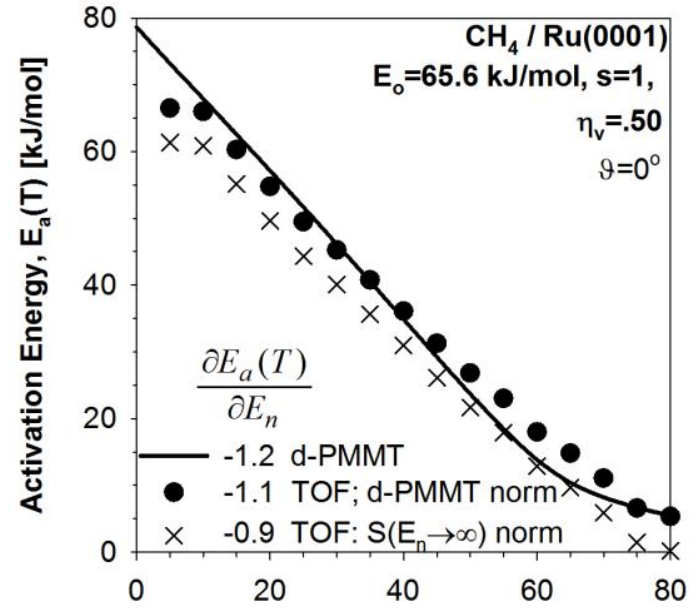

(b) Normal Translational Energy, $E_{n}[\mathrm{~kJ} / \mathrm{mol}]$

Figure 4.5. (a) Temperature dependent dissociative sticking coefficients, $S_{n}\left(E_{t}, T\right)$, (points) derived from the experimental associative desorption energy distributions of Fig. 3 are normalized by and compared to d-PMMT simulations (lines). (b) Thermal activation energies with respect to $\mathrm{T}$ are plotted as a function of $\mathrm{E}_{\mathrm{n}}$ for d-PMMT simulations, and from the experimentally derived $S_{n}\left(E_{t}, T\right)$ normalized by d-PMMT simulations of $S_{n}(T)$ (points) or the assumption $S_{n}\left(E_{t}=150 \mathrm{~kJ} / \mathrm{mol}, T\right) \rightarrow 1$ (crosses). 


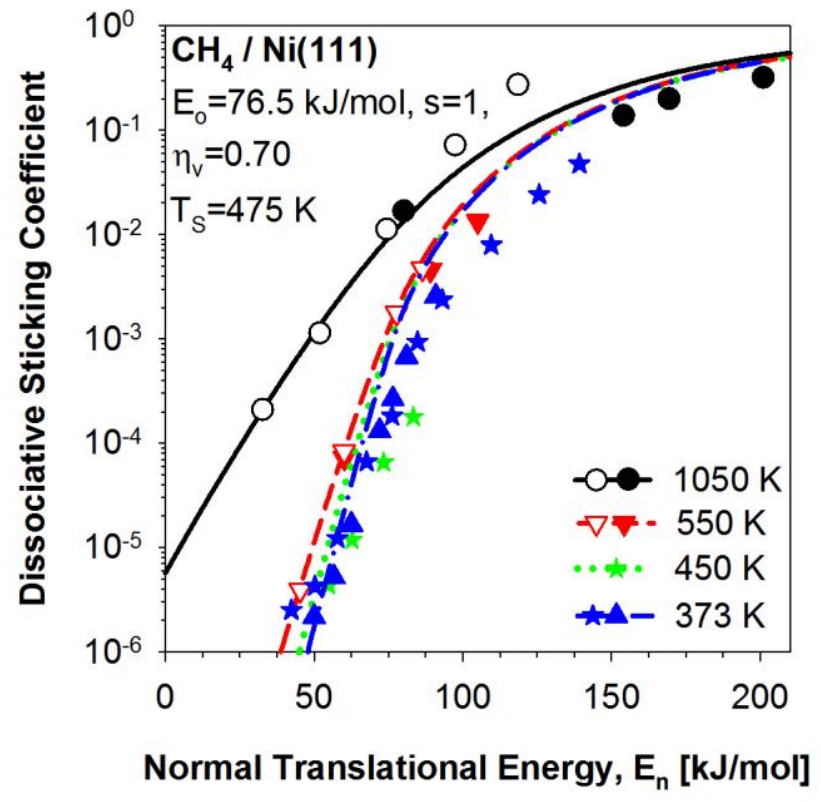

Figure 4.6. Dissociative sticking coefficients for supersonic molecular beams of $\mathrm{CH}_{4}$ incident on $\mathrm{Ni}(111)$. Experimental data (points) by Beck (stars), ${ }^{81} \mathrm{Utz}$ (all other filled), ${ }^{101}$ and Chorkendorff (open $)^{72}$ are compared to PMMT models (lines) whose parameters were optimized to the experiments by Utz and Chorkendorff. 

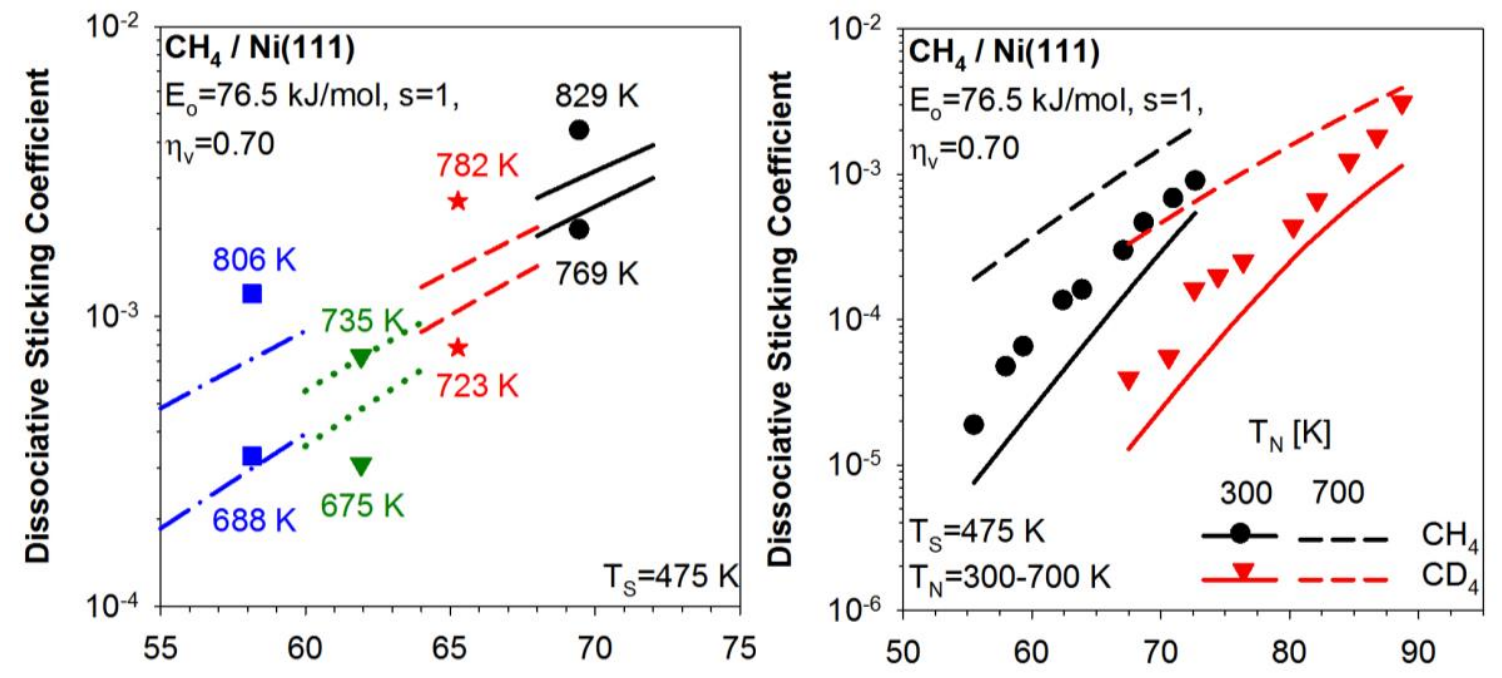

(a) Normal Translational Energy, $\mathrm{E}_{\mathrm{n}}[\mathrm{kJ} / \mathrm{mol}]$ (b)

Normal Translational Energy, $\mathrm{E}_{\mathrm{n}}[\mathrm{kJ} / \mathrm{mol}]$

Figure 4.7. d-PMMT dissociative sticking coefficients (lines) are compared to Ceyer's experimental measurements (points) on $\mathrm{Ni}(111)$ for (a) a range of nozzle temperatures and translational energies and (b) the two isotopomers of methane with neat beams at varying nozzle temperatures ${ }^{77,110}$. The d-PMMT predictions in (b) are given for the $300 \mathrm{~K}$ (solid) and $700 \mathrm{~K}$ (dashed) bounds on the experimental $\mathrm{T}_{\mathrm{N}}$. 

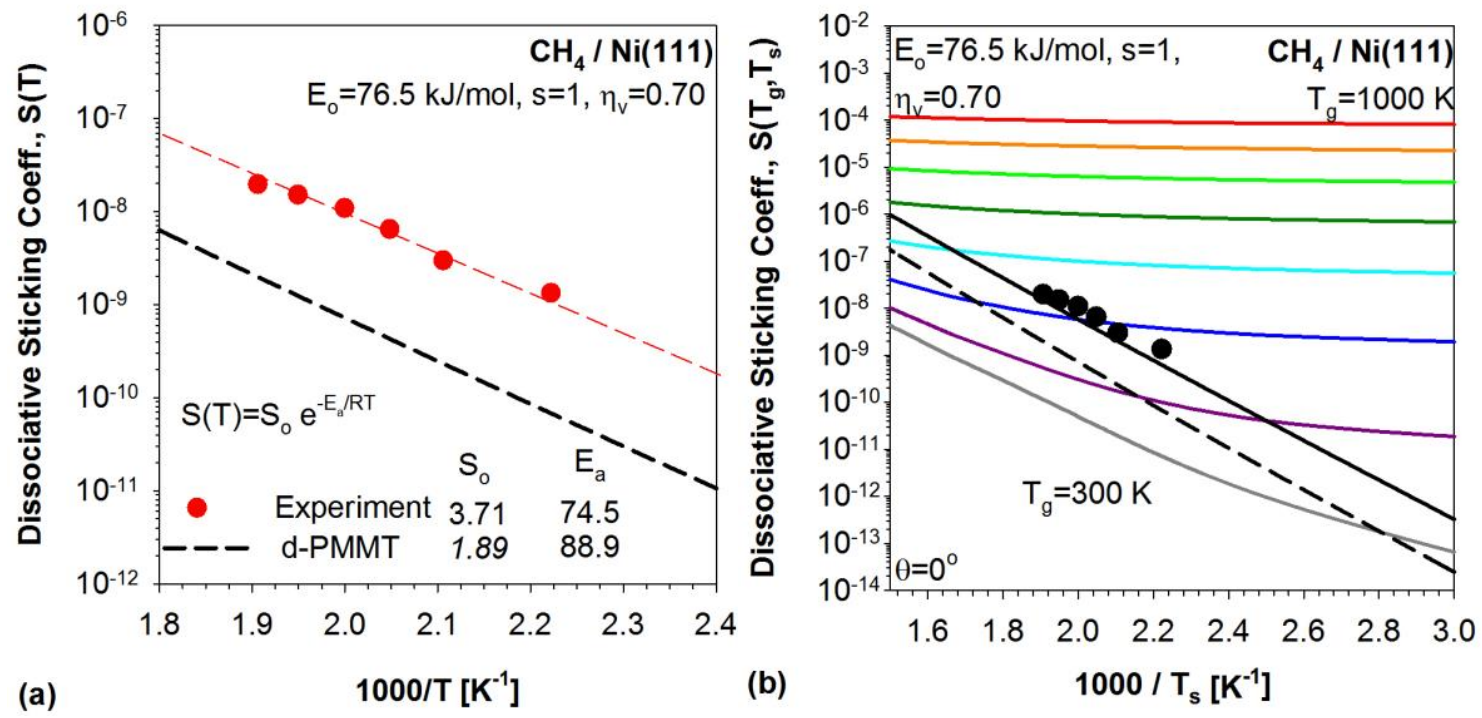

Figure 4.8. d-PMMT simulations for normally incident (solid lines) and angle-integrated (dashed lines) dissociative sticking coefficients are compared to experiments (points) ${ }^{32}$ for $\mathrm{CH}_{4} / \mathrm{Ni}(111)$ under both thermal equilibrium and nonequilibrium conditions. (a) The red dashed line is an Arrhenius fit to the experimental $S(T)$. (b) $S_{n}\left(T_{g}, T_{s}\right)$ are given as solid lines for gas temperatures from $\mathrm{T}_{\mathrm{g}}=300 \mathrm{~K}$ to $1000 \mathrm{~K}$ in increments of $100 \mathrm{~K}$. 


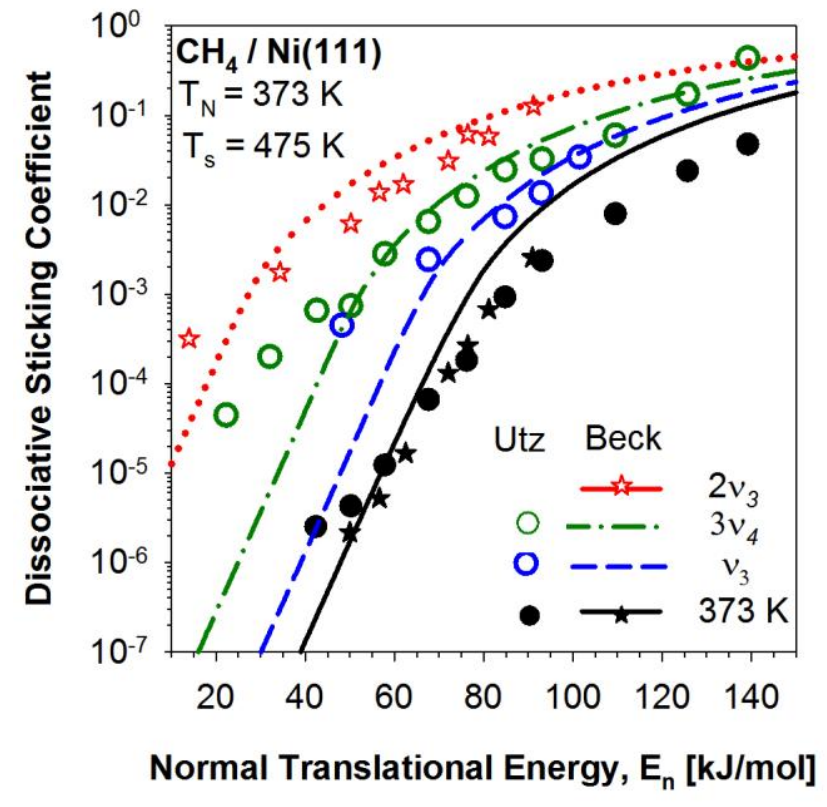

Figure 4.9. Experimental (points) dissociative sticking coefficients for $\mathrm{CH}_{4}$ supersonic molecular beams incident on $\mathrm{Ni}(111)$ are compared to d-PMMT values (lines) for dissociative sticking

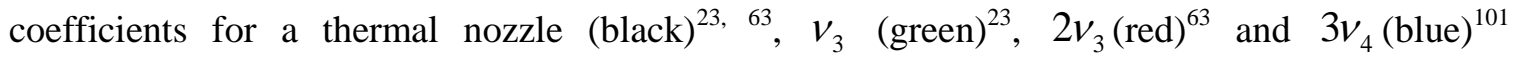
eigenstates. 


\begin{tabular}{lllll}
\hline State & $\begin{array}{l}E_{\text {vib }} \\
{[\mathrm{kJ} / \mathrm{mol}]}\end{array}$ & Surface & $\begin{array}{l}\Delta E_{\text {trans }} \\
{[\mathrm{kJ} / \mathrm{mol}]}\end{array}$ & $\eta_{\text {vib,exp }}$ \\
\hline d-PMMT & averaged & $\mathrm{Ni}(100)$ & & 0.72 \\
$v_{3}{ }^{27}$ & 36 & $\mathrm{Ni}(100)$ & 34 & 0.94 \\
$2 v_{3}{ }^{24}$ & 71 & $\mathrm{Ni}(100)$ & 68 & 0.96 \\
$v_{1}{ }^{17}$ & 35 & $\mathrm{Ni}(100)$ & 50 & 1.4 \\
$3 v_{4}{ }^{23}$ & 45 & $\mathrm{Ni}(100)$ & - & $<0.5$ \\
$\mathrm{~d}-\mathrm{PMMT}$ & averaged & $\mathrm{Ni}(111)$ & & 0.70 \\
$v_{3}{ }^{111}$ & 36 & $\mathrm{Ni}(111)$ & 45 & 1.24 \\
$2 v^{63}$ & 71 & $\mathrm{Ni}(111)$ & 65 & .90 \\
$3 v_{4}{ }^{23}$ & 45 & $\mathrm{Ni}(111)$ & 34 & .72 \\
$\mathrm{~d}-\mathrm{PMMT}$ & averaged & $\mathrm{Pt}(111)$ & & 0.40 \\
$2 v^{63}{ }^{63,112}$ & 71 & $\mathrm{Pt}(111)$ & 28 & 0.4 \\
\hline
\end{tabular}

Table 4.1. Eigenstate specific vibrational efficacies calculated from supersonic beam methane dissociative chemisorption experiments are compared to d-PMMT thermal averaged vibrational efficacies for the dissociative chemisorption of methane over three different single crystal metal surfaces. 


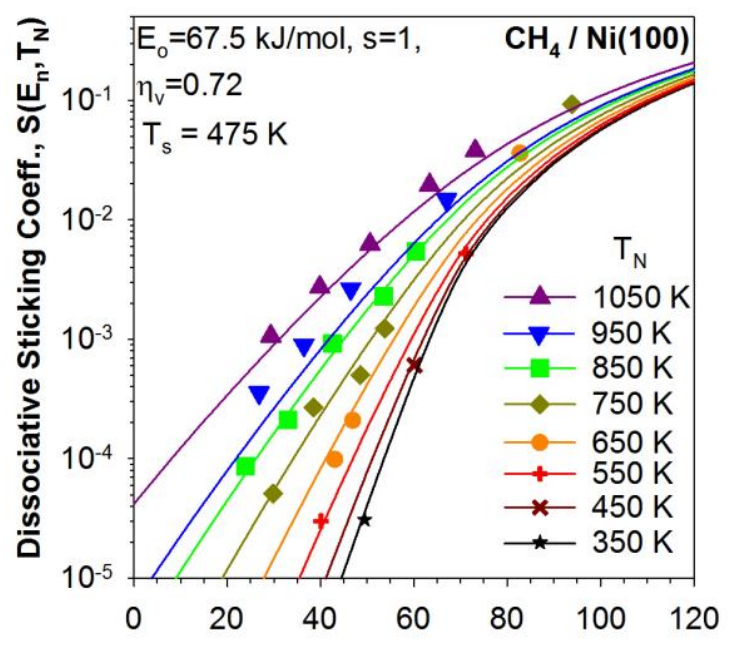

(a) Normal Translational Energy, $E_{n}[\mathrm{~kJ} / \mathrm{mol}]$

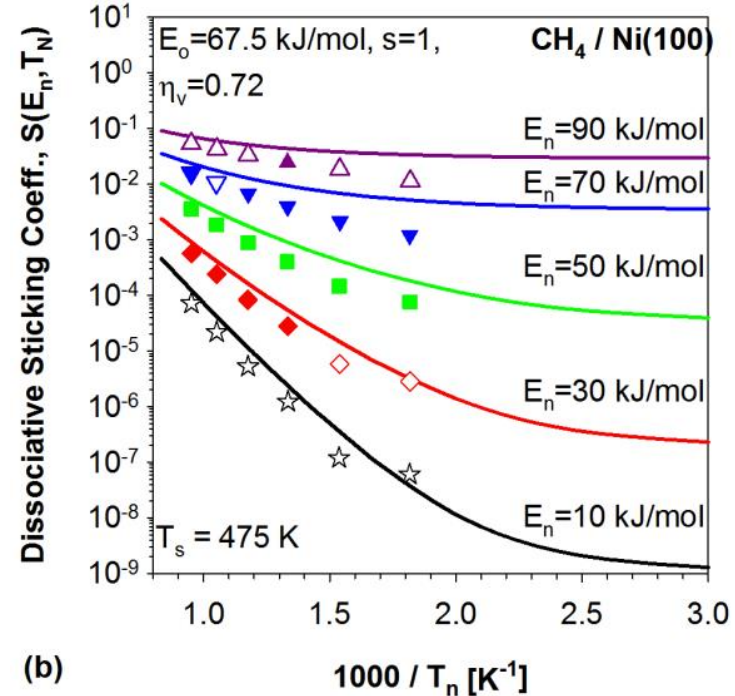

Figure 4.10. d-PMMT theoretical simulations (lines) are compared to supersonic molecular beam experiments $^{28}$ (points) for methane dissociative chemisorption on $\mathrm{Ni}(100)$ for a large range of nozzle temperatures. Slices at specific normal translational energies through smooth empirical fits drawn by $\mathrm{Holmblad}^{28}$ through the experimental points of (a), determined either by interpolation (solid) or extrapolation (open) are plotted against d-PMMT predictions for reactivity as a function of nozzle temperature. 

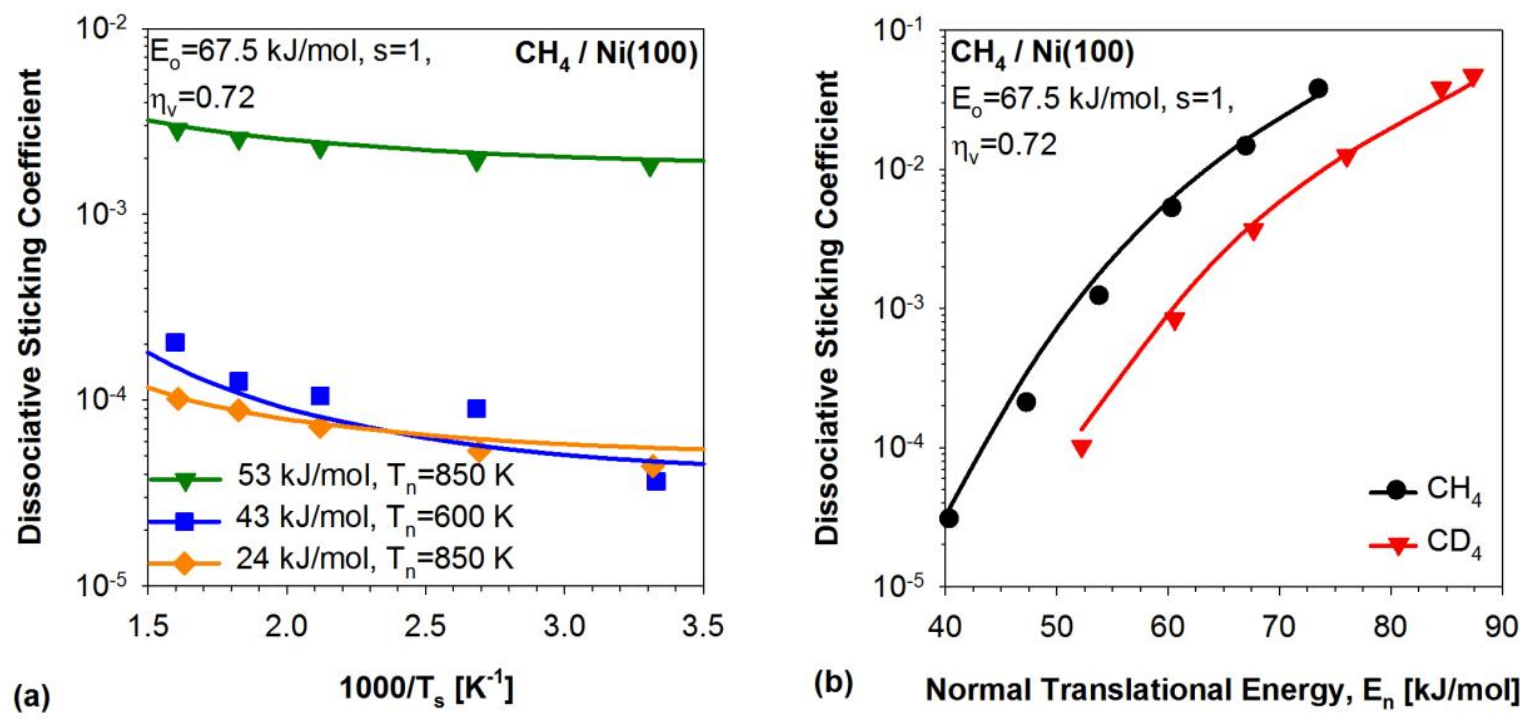

(b) Normal Translational Energy, $E_{n}[\mathrm{~kJ} / \mathrm{mol}]$

Figure 4.11. Experimental (points) from Holmblad ${ }^{28}$ and d-PMMT theoretical predictions of dissociative sticking coefficients as a function of (a) surface temperature at varying translationand vibrational energies and (b) normal translational energy over a range of nozzle temperatures for supersonic molecular beams of $\mathrm{CH}_{4}$ isotopomers incident on $\mathrm{Ni}(100)$. 


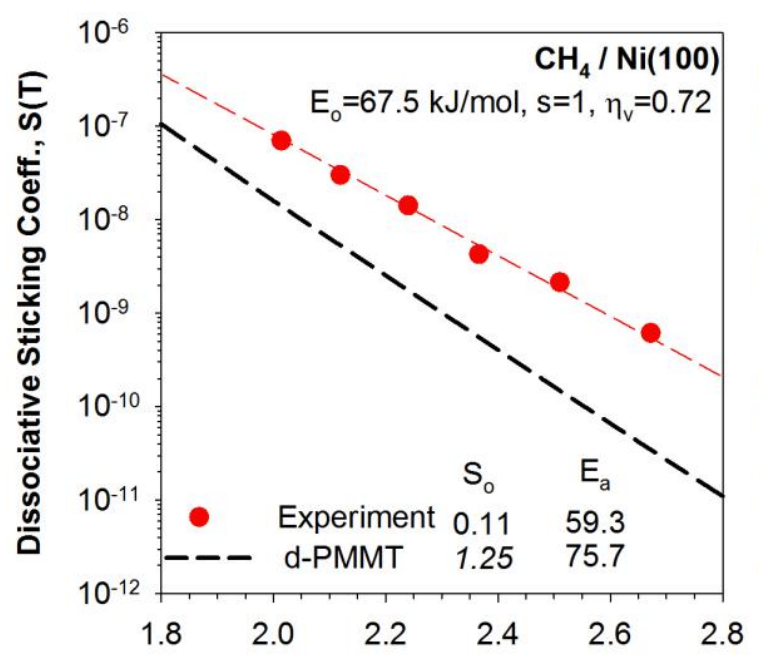

(a)

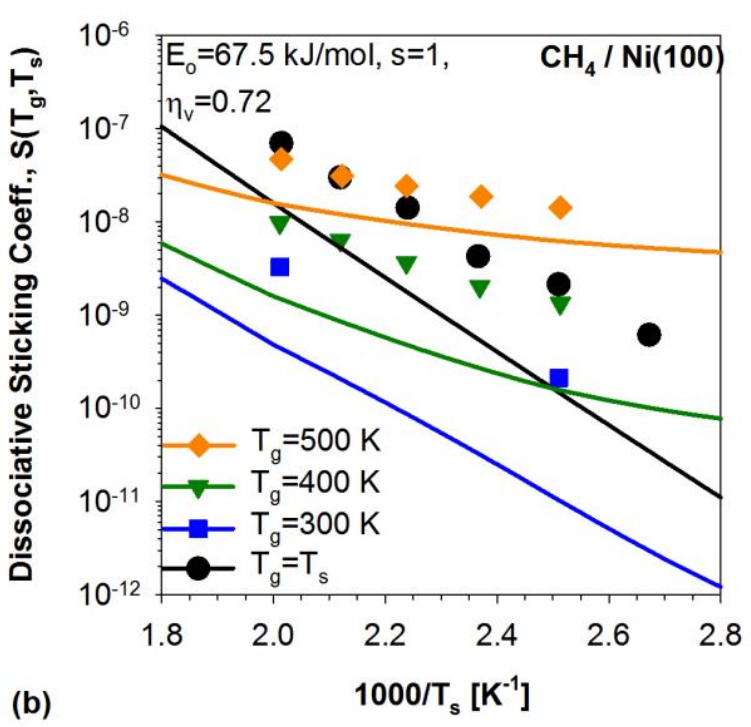

Figure 4.12. Thermal dissociative sticking coefficients reported by Nielsen ${ }^{19}$ for methane dissociative chemisorption on $\mathrm{Ni}(100)$ are compared to d-PMMT simulations. The sticking coefficients shown in (b) include both equilibrium, $\mathrm{T}_{\mathrm{g}}=\mathrm{T}_{\mathrm{s}}$, (black) on non-equilibrium (colored) measurements. 


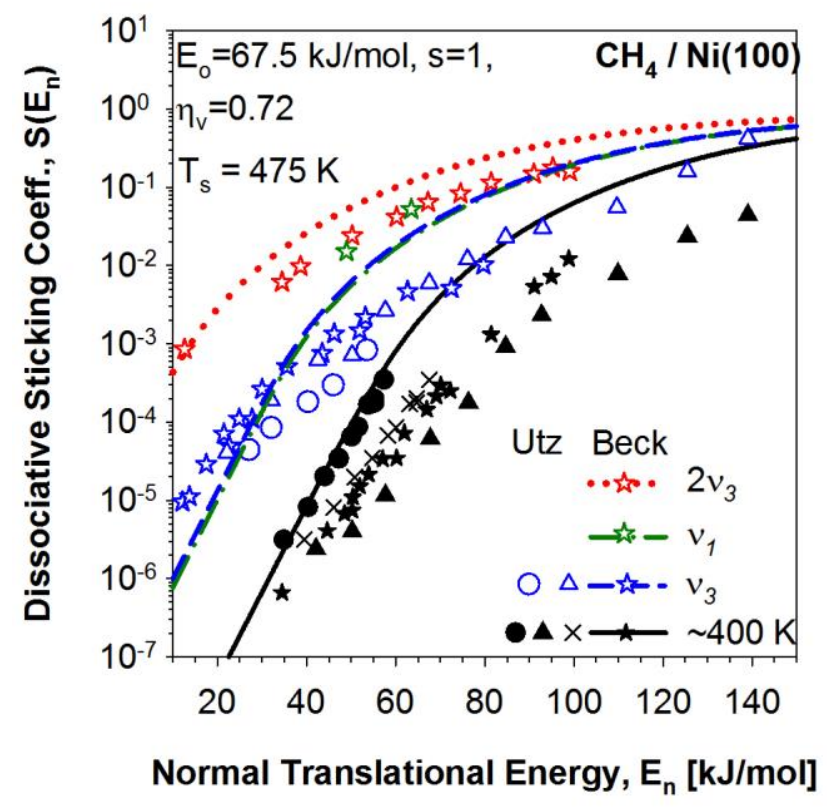

Figure 4.13. Experiments ${ }^{21,23-25,27,58,111,113}$ (points) are compared to d-PMMT theoretical models (lines) for dissociative sticking coefficients for a thermal nozzle (black), $v_{1}$ (blue), $2 v_{3}$ (red) and $v_{3}$ (green) eigenstate-resolved supersonic molecular beam of $\mathrm{CH}_{4}$ incident on $\mathrm{Ni}(100)$. 

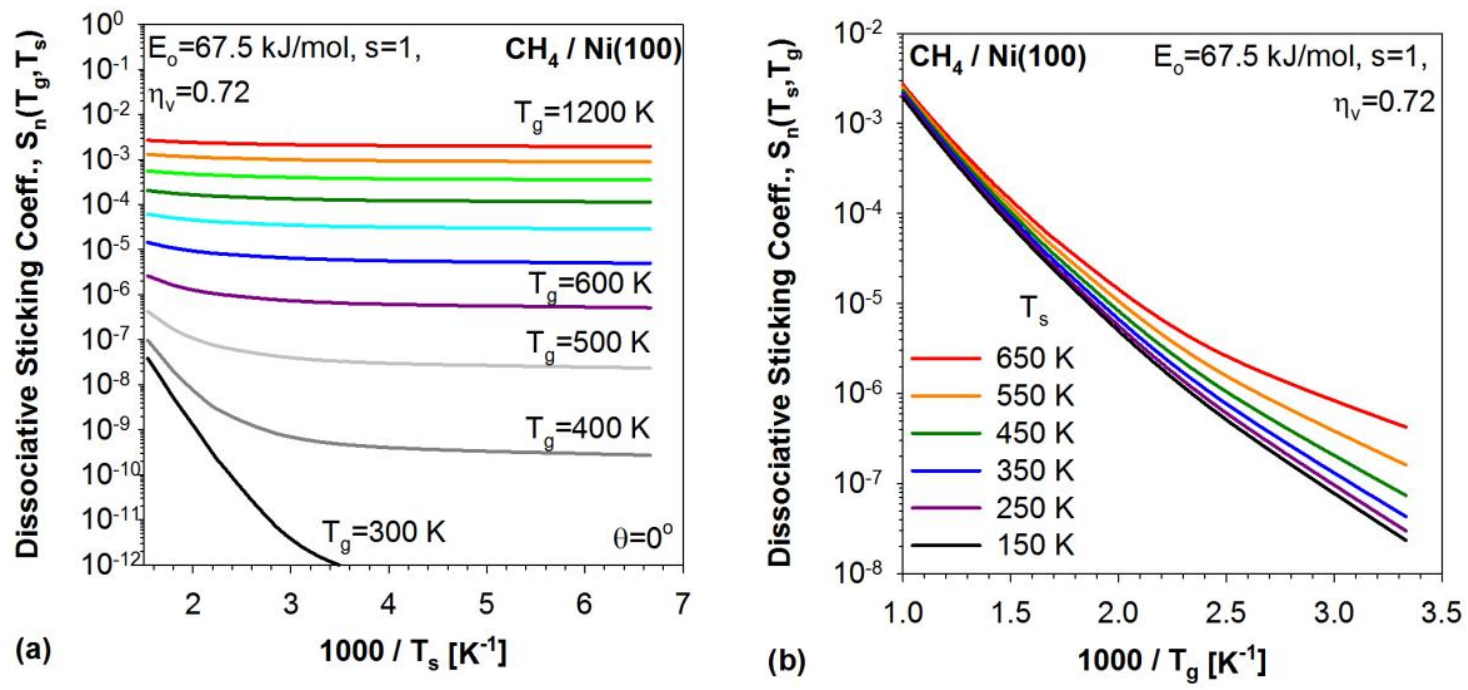

Figure 4.14. d-PMMT simulations of the dissociative sticking coefficient from a normally incident thermal effusive beam of methane on $\mathrm{Ni}(100)$ as a function of (a) surface and (b) gas temperature. 


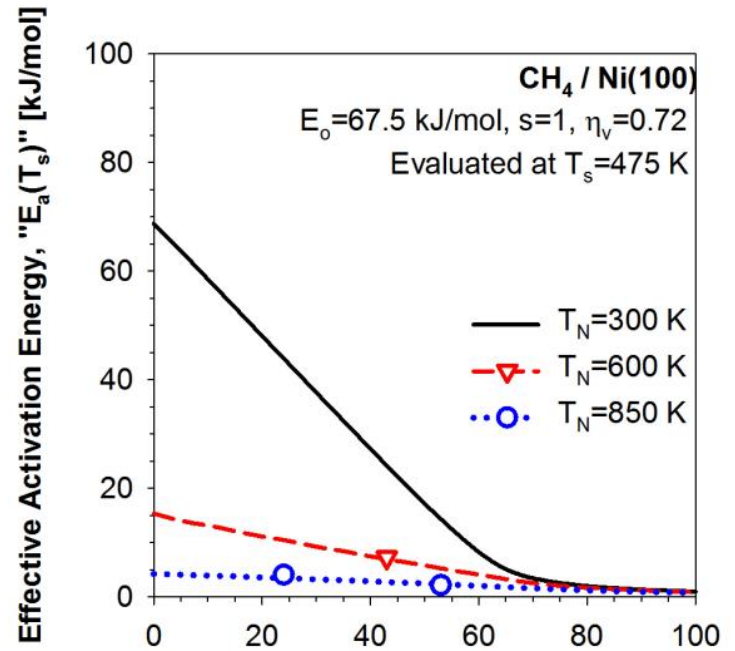

(a) Normal Translational Energy, $E_{n}[\mathrm{~kJ} / \mathrm{mol}]$

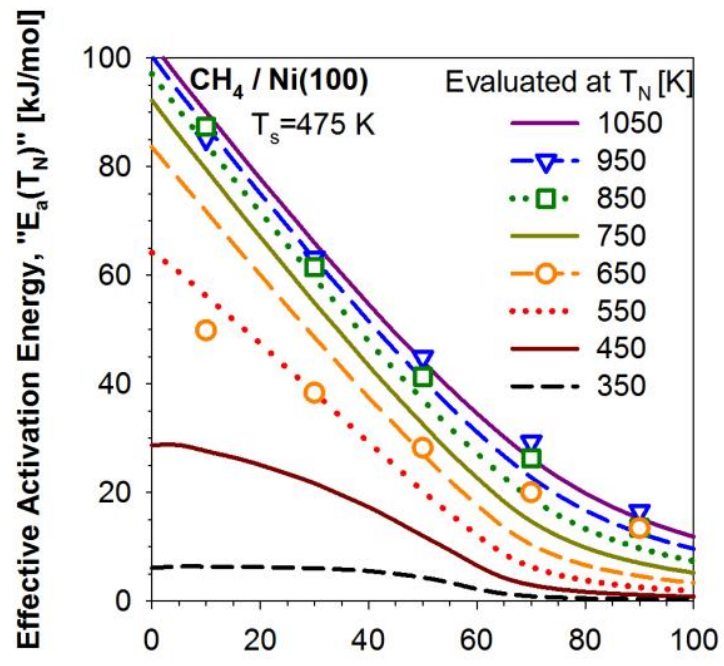

(b)

Normal Translational Energy, $\mathrm{E}_{\mathrm{n}}[\mathrm{kJ} / \mathrm{mol}]$

Figure 4.15. Effective activation energies for (a) surface and (b) vibrational temperature, $k_{B} T_{j}^{2} \partial \ln S\left(T_{j}\right) / \partial T_{j}=" E_{a}\left(T_{j}\right) "=\left(\left\langle E_{j}\right\rangle_{R}-\left\langle E_{j}\right\rangle\right) / \eta_{v}$, calculated from the Holmblad supersonic molecular beam data of Figs. 4.10(a) and 4.11(a) are compared to d-PMMT model predictions (lines). Experimentally based values (points) were calculated based on the $\ln S\left(T_{j}\right)$ data points straddling the evaluation temperatures. 


\begin{tabular}{ccc}
\hline $\begin{array}{c}\text { Evaluated at } \\
\mathrm{T}_{\mathrm{N}}=[\mathrm{K}]\end{array}$ & \multicolumn{2}{c}{$\partial " E_{a}\left(T_{N}\right) " / \partial E_{n}$} \\
$\mathrm{E}_{\mathrm{n}}=40 \mathrm{~kJ} / \mathrm{mol}$ & Expt. & d-PMMT \\
\hline 350 & & -0.09 \\
450 & & -0.48 \\
550 & -0.51 & -0.91 \\
650 & -1.01 & -1.08 \\
750 & -1.12 \\
850 & & -1.12 \\
950 & -0.92 & -1.11 \\
1050 & -0.98 & -1.09 \\
\hline
\end{tabular}

Table 4.2. Experimental slopes of change in effective activation energy, " $E_{a}\left(T_{N}\right)$ ", with respect to normal translational energy calculated from the points shown in Fig. 4.15 for $\mathrm{CH}_{4} / \mathrm{Ni}(100)$ evaluated at $E_{n}=40 \mathrm{~kJ} / \mathrm{mol}$ over a range of nozzle temperatures, $T_{N}$, are compared to those calculated from d-PMMT simulations. 


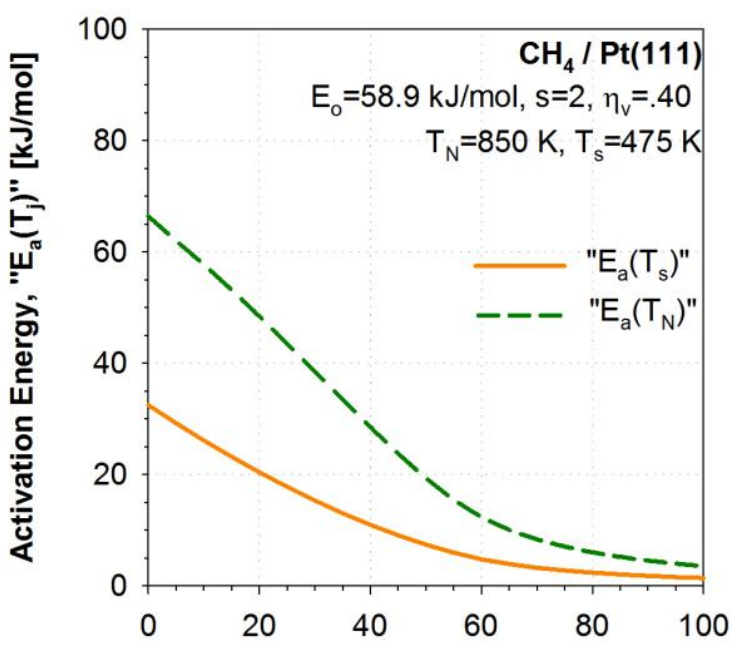

(a) Normal Translational Energy, $E_{n}[\mathrm{~kJ} / \mathrm{mol}]$

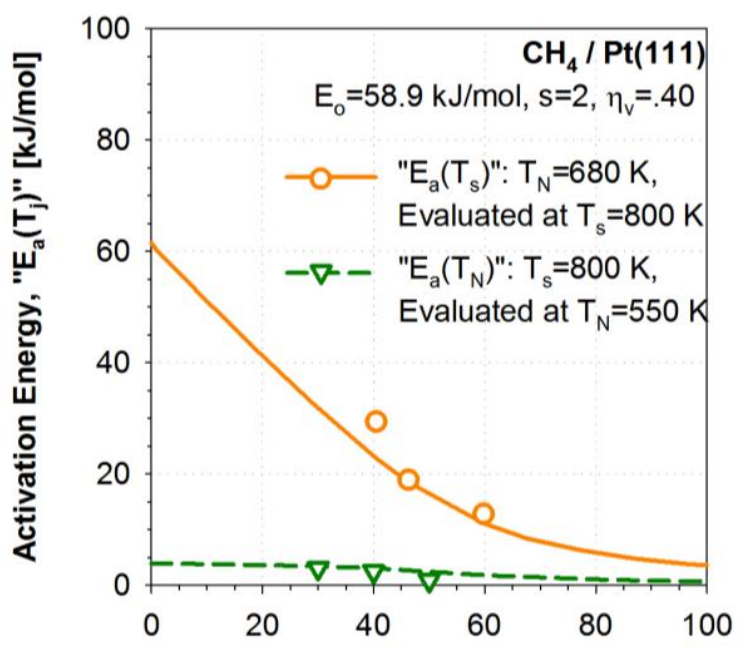

(c) Normal Translational Energy, $E_{n}[\mathrm{~kJ} / \mathrm{mol}]$

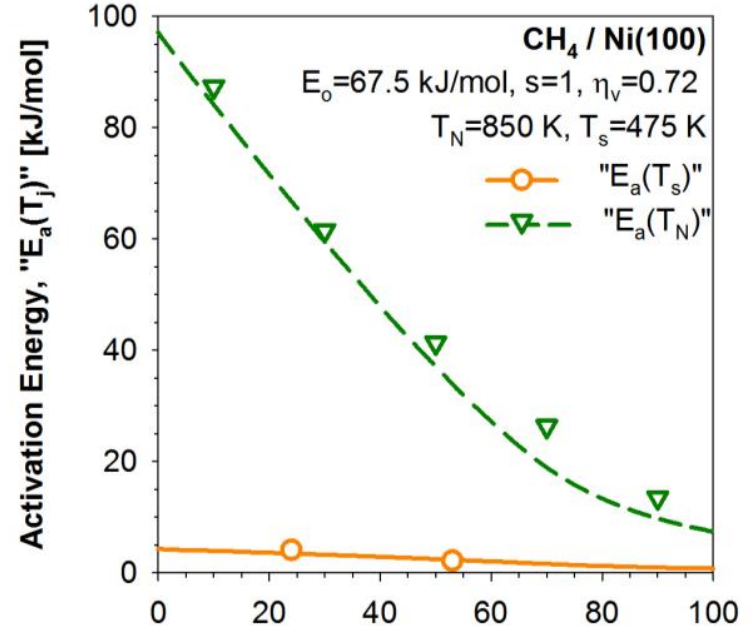

(b) Normal Translational Energy, $\mathrm{E}_{\mathrm{n}}[\mathrm{kJ} / \mathrm{mol}]$

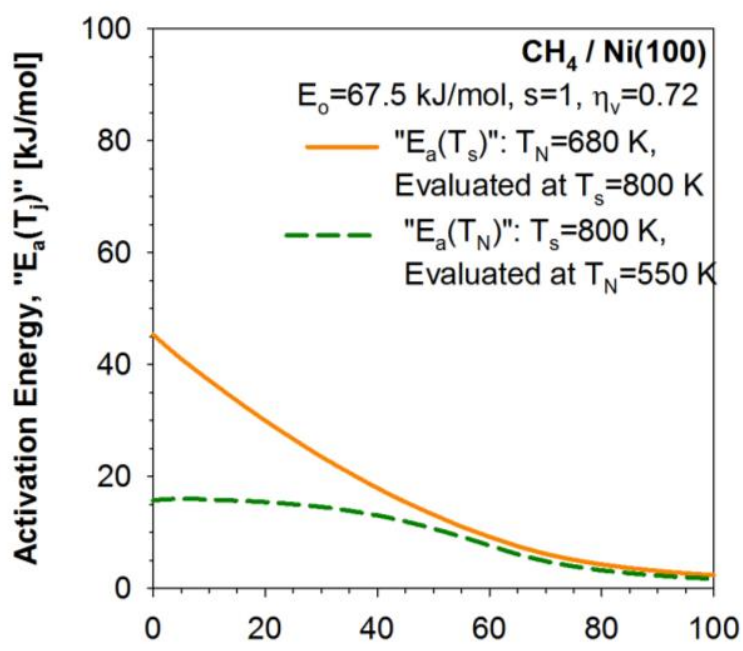

(d) Normal Translational Energy, $\mathrm{E}_{\mathrm{n}}[\mathrm{kJ} / \mathrm{mol}]$

Figure 4.16. Comparison between effective activation energies for methane dissociative chemisorption on (a and c) $\mathrm{Pt}(111)^{8,}, 96$ and (b and d) $\mathrm{Ni}(100)^{28}$ as a function of normal translational energy for d-PMMT models (lines) and experiments (points). 


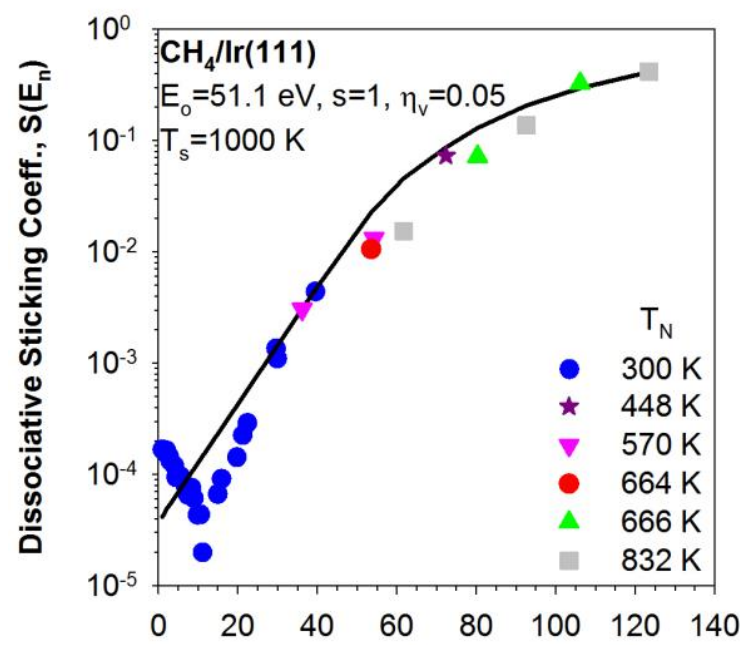

(a) Normal Translational Energy, $E_{n}[\mathrm{~kJ} / \mathrm{mol}]$

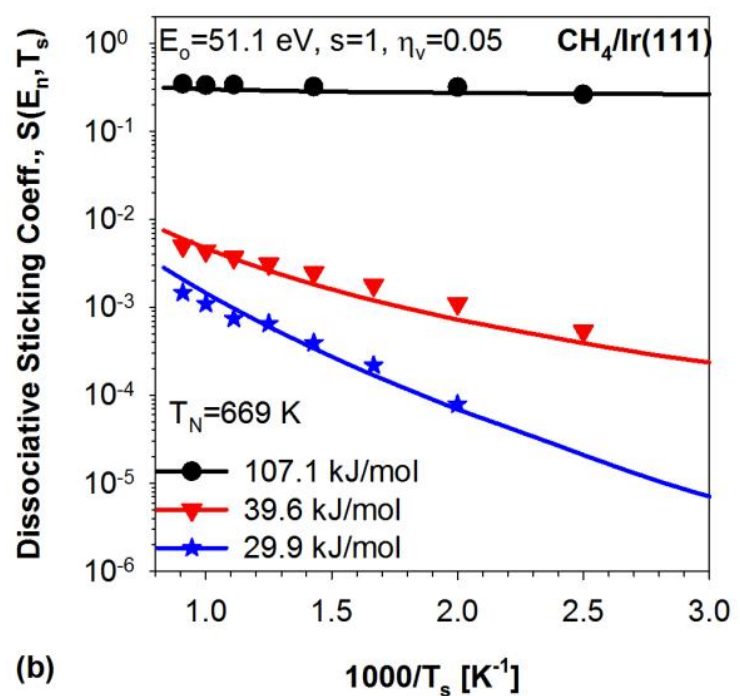

Figure 4.17. Initial dissociative sticking coefficients for $\mathrm{CH}_{4}$ on $\operatorname{Ir}(111)$ as a function of (a) normal translational energy and (b) surface temperature. Sticking derived from the molecular beam experiments of Seets ${ }^{10}$ (closed symbols) are compared to d-PMMT predictions (lines). 

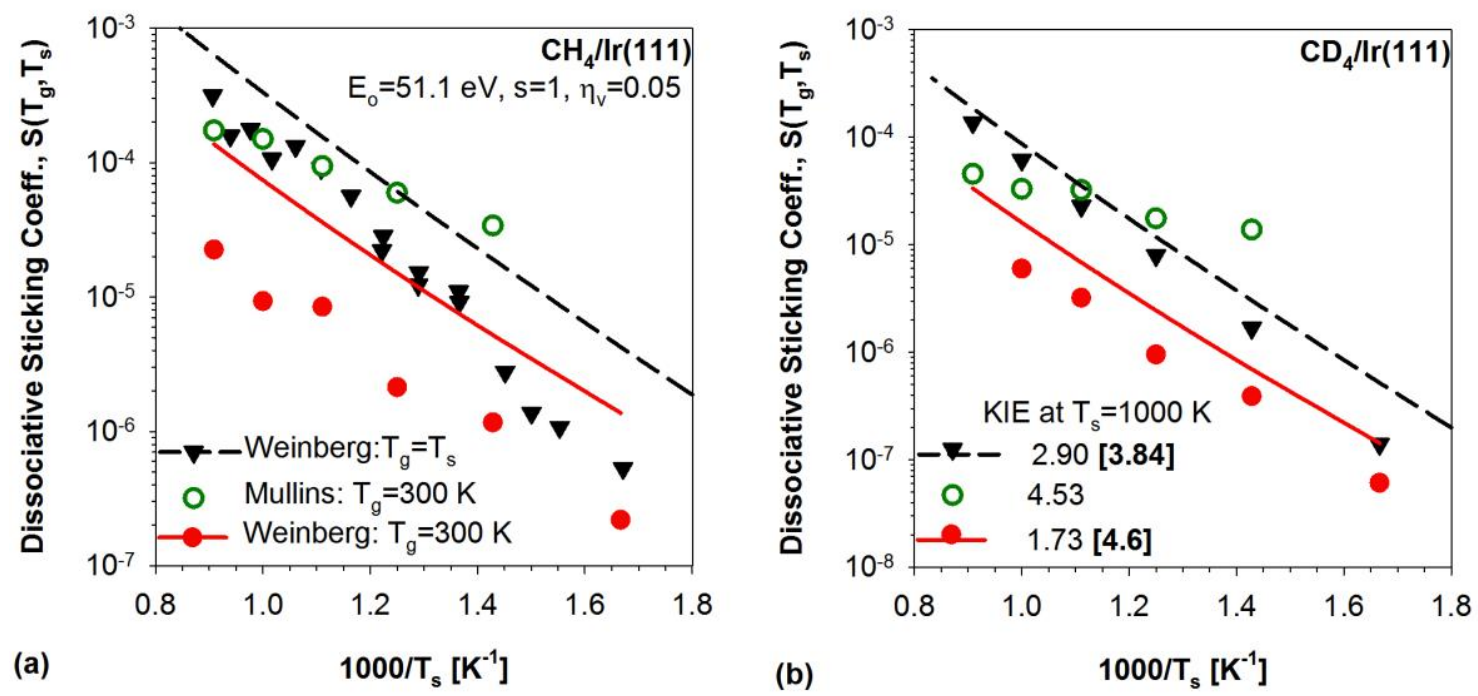

Figure 4.18. Intermediate pressure experimental dissociative sticking (points) for (a) $\mathrm{CH}_{4}$ and (b) $\mathrm{CD}_{4}$ on $\operatorname{Ir}(111)^{10,11}$ are compared to d-PMMT calculations (lines). Kinetic isotope effects, $\mathrm{KIE}=\mathrm{S}\left(\mathrm{CH}_{4}\right) / \mathrm{S}\left(\mathrm{CD}_{4}\right)$, are given for experiments and the d-PMMT model (bold). 


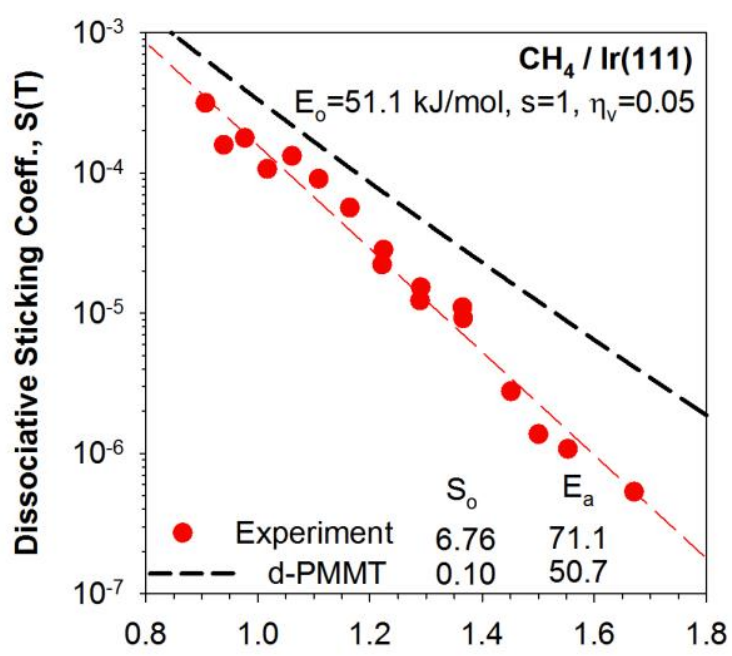

(a)
$1000 / \mathrm{T}\left[\mathrm{K}^{-1}\right]$

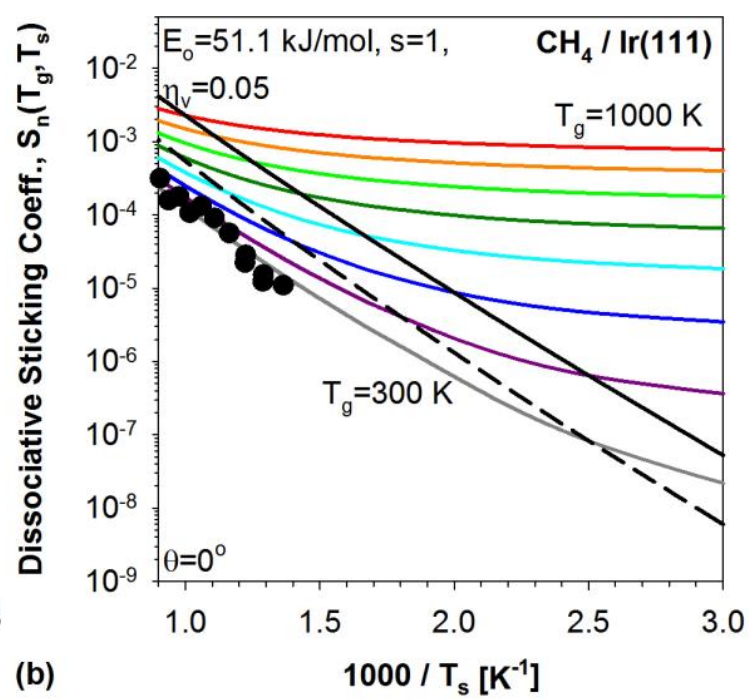

Figure 4.19. d-PMMT simulations for normally incident (solid lines) and angle-integrated (dashed lines) dissociative sticking coefficients are compared to experiments by Weinberg ${ }^{11}$ (points) ${ }^{32}$ for $\mathrm{CH}_{4} / \mathrm{Ir}(111)$ under both thermal equilibrium and nonequilibrium conditions. (a) The red dashed line is an Arrhenius fit to the experimental $\mathrm{S}(\mathrm{T})$. (b) $\mathrm{S}_{\mathrm{n}}\left(\mathrm{T}_{\mathrm{g}}, \mathrm{T}_{\mathrm{s}}\right)$ are given as solid lines for gas temperatures from $\mathrm{T}_{\mathrm{g}}=300 \mathrm{~K}$ to $1000 \mathrm{~K}$ in increments of $100 \mathrm{~K}$. 


\begin{tabular}{|c|c|c|c|c|c|c|}
\hline & $\operatorname{Pt}(111)$ & $\mathrm{Ni}(111)$ & $\mathrm{Ni}(100)$ & $\mathrm{Ru}(0001)$ & $\operatorname{Ir}(111)$ & $\begin{array}{c}\text { Gas } \\
\text { Phase }\end{array}$ \\
\hline $\mathrm{E}_{0}[\mathrm{~kJ} / \mathrm{mol}]$ & 58.9 & 76.5 & 67.5 & 65.6 & 48.2 & \\
\hline s & 2 & 1 & 1 & 1 & 1 & \\
\hline $\mathrm{h}_{\mathrm{v}}$ & .40 & .70 & .72 & .50 & .30 & \\
\hline $\mathrm{h}_{\mathrm{s}}$ & 1 & 1 & 1 & 1 & .70 & \\
\hline $\mathrm{S}(\mathrm{T}=873 \mathrm{~K})$ & $1.22 * 10^{-4}$ & $1.12 * 10^{-5}$ & $7.23 * 10^{-5}$ & $3.02 * 10^{-5}$ & $2.14 * 10^{-4}$ & \\
\hline Z-H & 1.246 & 1.046 & 1.082 & & & - \\
\hline $\mathrm{Z}-\mathrm{C}$ & 2.257 & 2.132 & 2.063 & & 2.27 & - \\
\hline H-C-H Angle & 132.6 & 133.5 & 127.44 & & 140 & 109.5 \\
\hline $\mathrm{C}-\mathrm{H}$ & 1.493 & 1.579 & 1.614 & & 1.43 & 1.09 \\
\hline Force on & .966 & 1.105 & 1.211 & & & - \\
\hline Atom & & & & & & \\
\hline
\end{tabular}

Z-H: Height of dissociating $\mathrm{H}$ atom above metal surface in Angstroms

Z-C: Height of $\mathrm{C}$ atom above metal surface in Angstroms

$\mathrm{C}-\mathrm{H}$ : Length of dissociating bond in Angstroms

Table 4.2. List of d-PMMT and GGA-DFT transition state parameters for single crystal transition metal surfaces. 

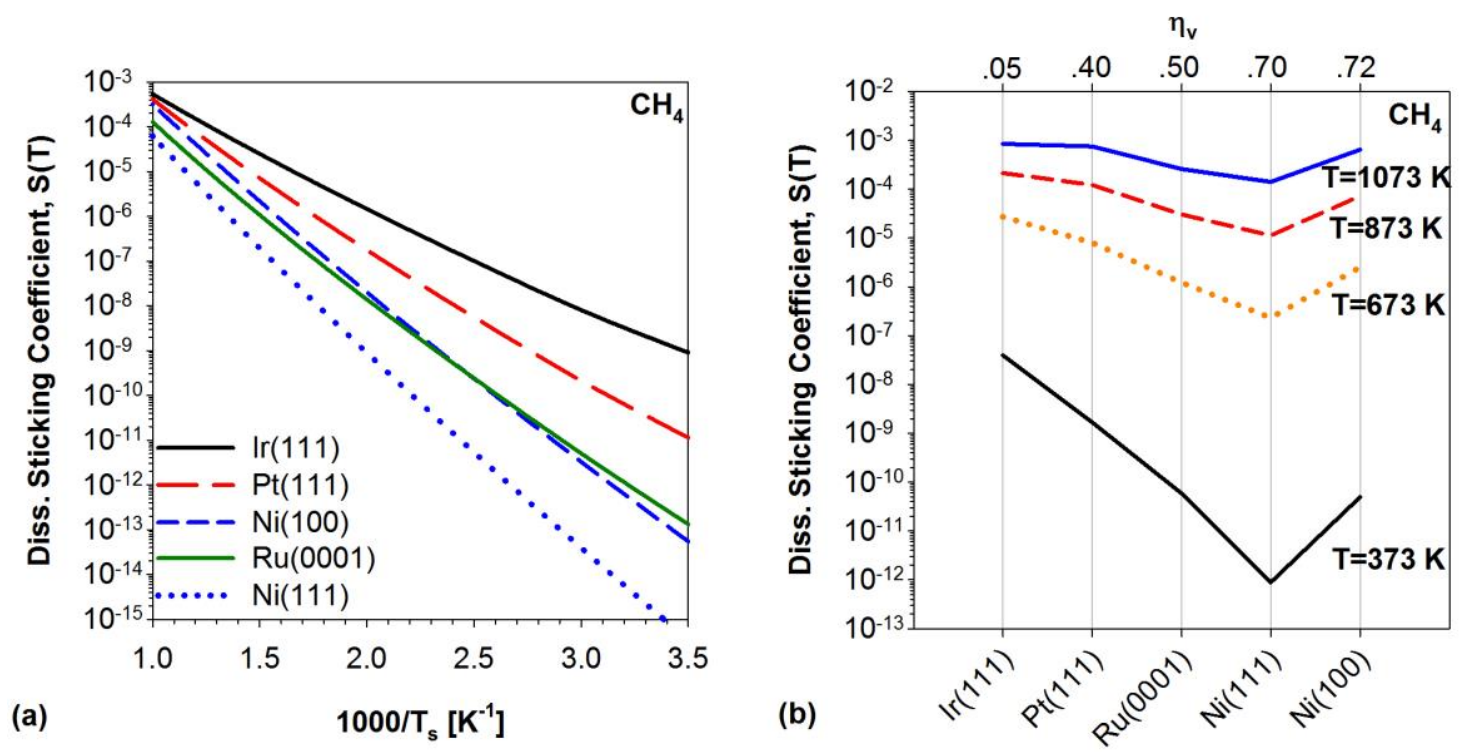

Figure 4.20. Comparison of dissociative sticking coefficients for a thermal ambient $\mathrm{CH}_{4}$ gas in equilibrium with the surface temperature on single crystal transition metal surfaces calculated by d-PMMT simulations. 

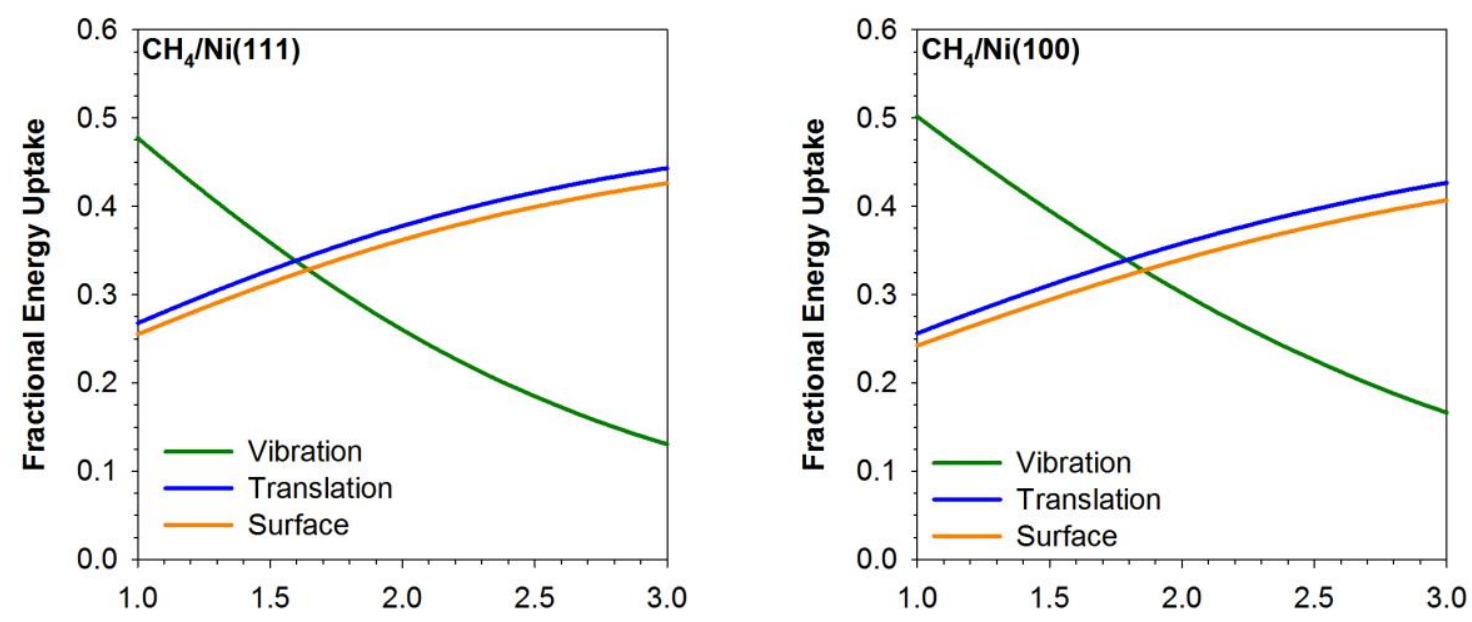

(a) $1000 / \mathrm{T}_{\mathrm{s}}\left[\mathrm{K}^{-1}\right]$

(b) $1000 / \mathrm{T}_{\mathrm{s}}\left[\mathrm{K}^{-1}\right]$
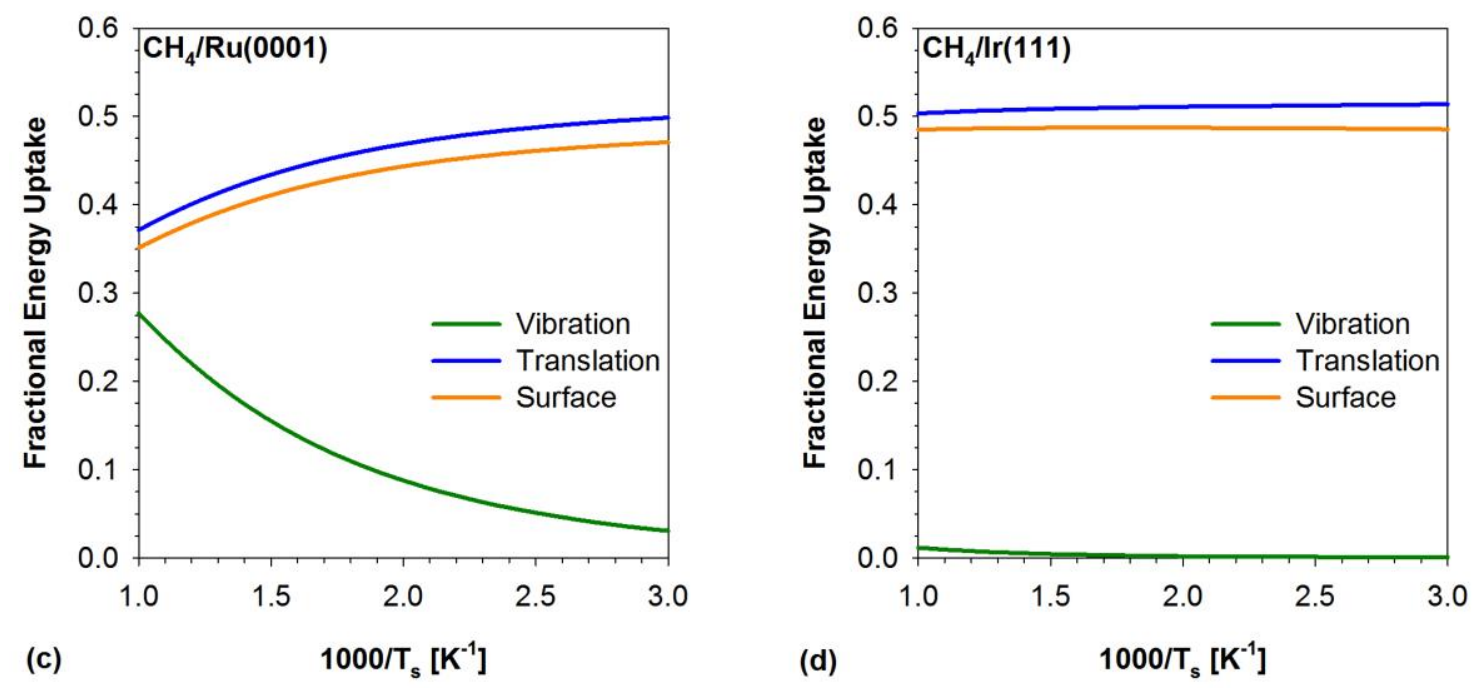

Figure 4.21. Fractional exchangeable energy uptakes, $f_{j}=\left\langle E_{j}\right\rangle_{R} /\left\langle E^{*}\right\rangle_{R}$ for $j \neq v$ and $f_{v}=\eta_{v}\left\langle E_{j}\right\rangle_{R} /\left\langle E^{*}\right\rangle_{R}$, for thermal dissociative chemisorption, where $\left\langle E_{j}\right\rangle_{R}$ and $\left\langle E^{*}\right\rangle_{R}$ are the mean energies derived from the $j^{\text {th }}$ degrees of freedom and the mean exchangeable energy, respectively, for the successfully reacting PCs. 

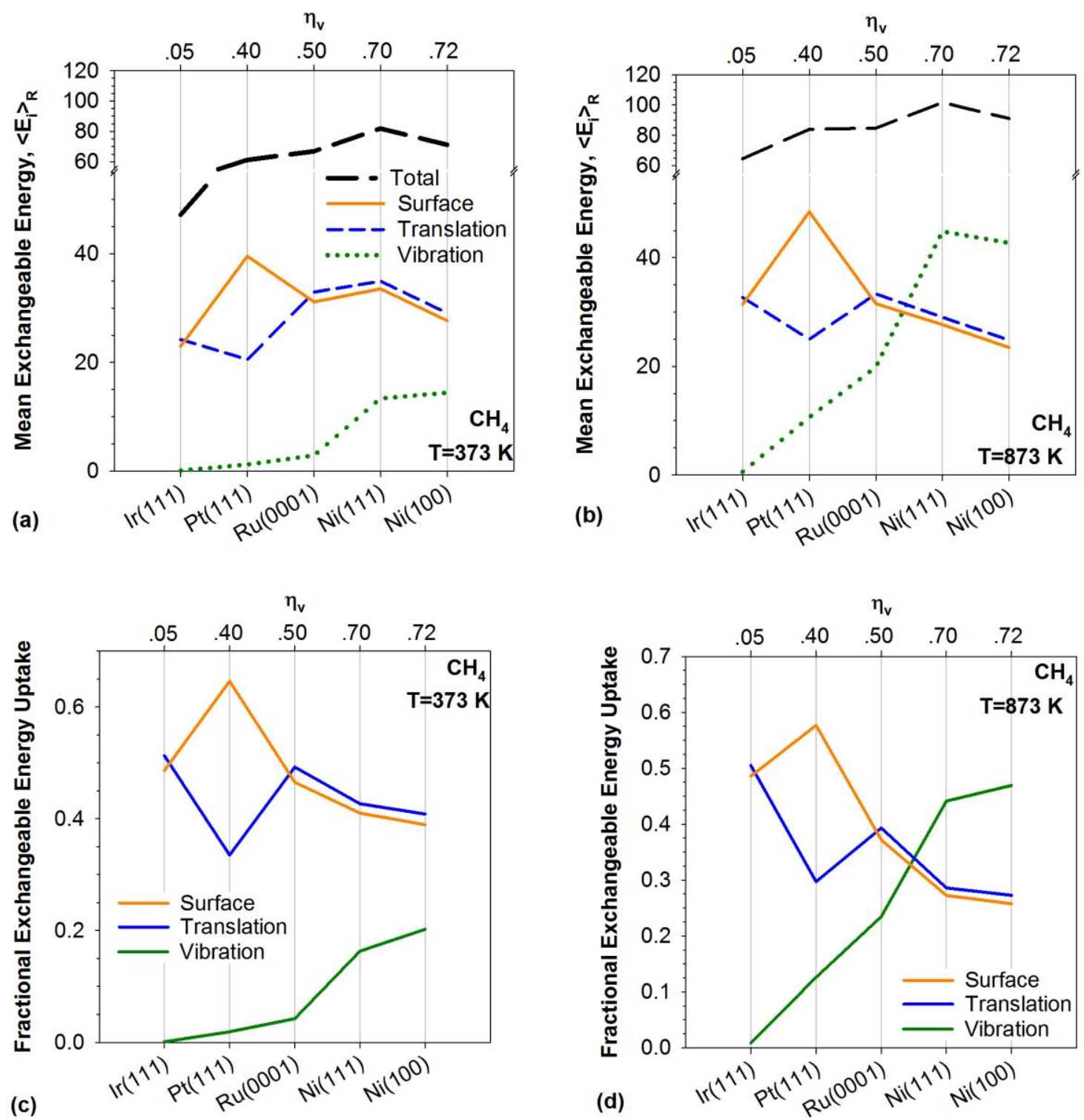

Figure 4.22. Mean exchangeable ( $a \& b)$ and fractional exchangeable (c \& d) energy uptakes for thermal dissociative chemisorption at 373 and $873 \mathrm{~K}$ for transition metal single crystals. 


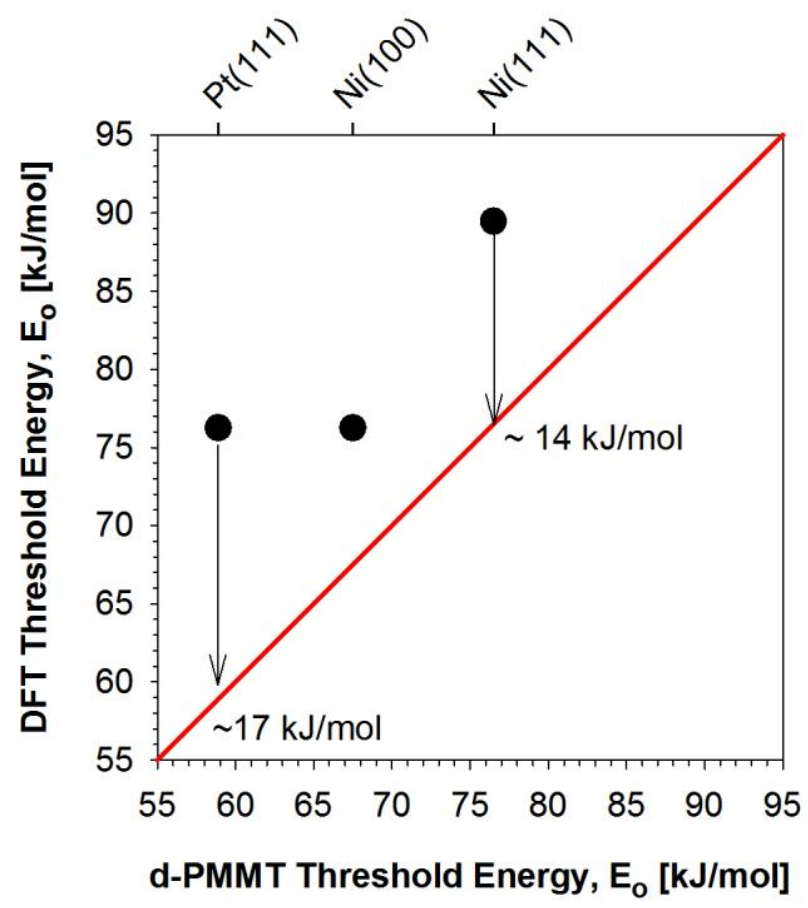

Figure 4.23. Comparison of apparent threshold energies from d-PMMT simulations to those calculated by GGA-DFT with the BPE functional ${ }^{14}$. The inset numbers describe the absolute difference between the theoretical energies. The adsorption energy of van der Waals bound $\mathrm{CH} 4$ on $\operatorname{Pt}(111)$ is $\mathrm{E}_{\mathrm{ad}}=15.7 \mathrm{~kJ} / \mathrm{mol}^{30}$ 


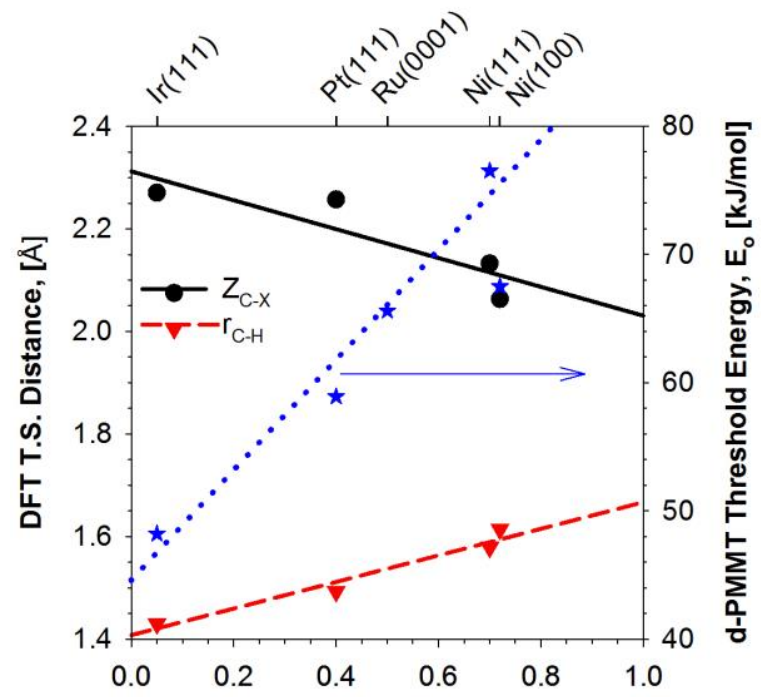

(a)

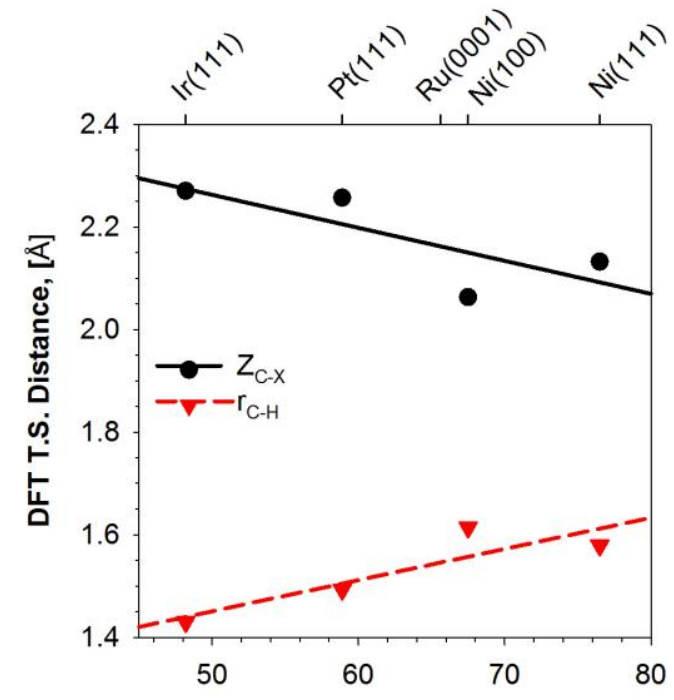

(b) d-PMMT Threshold Energy, $E_{o}[\mathrm{~kJ} / \mathrm{mol}]$

Figure 4.24. Transition state characteristics, distance of carbon atom to surface and length of dissociating C-H bond, from Jackson ${ }^{14}$ and Henkleman ${ }^{95}$ electronic structure theory calculations are related to d-PMMT efficacies of vibration (a) and threshold energies (b) of the dissociative chemisorption of methane on transition metals. Predicted $\mathrm{C}-\mathrm{H}$ and $\mathrm{C}-\mathrm{X}$ values for $\mathrm{Ru}(0001)$ are 1.5374 and 2.1714 angstroms respectively. 


\section{References:}

1. Wei, J. M.; Iglesia, E., Mechanism and site requirements for activation and chemical conversion of methane on supported Pt clusters and turnover rate comparisons among noble metals. J. Phys. Chem. B 2004, 108, (13), 4094-4103.

2. Wei, J. M.; Iglesia, E., Structural requirements and reaction pathways in methane activation and chemical conversion catalyzed by rhodium. J. Catal. 2004, 225, (1), 116-127.

3. Wei, J. M.; Iglesia, E., Reaction pathways and site requirements for the activation and chemical conversion of methane on Ru-based catalysts. J. Phys. Chem. B 2004, 108, (22), 72537262 .

4. Wei, J. M.; Iglesia, E., Isotopic and kinetic assessment of the mechanism of reactions of $\mathrm{CH}_{4}$ with $\mathrm{CO}_{2}$ or $\mathrm{H}_{2} \mathrm{O}$ to form synthesis gas and carbon on nickel catalysts. J. Catal. 2004, 224, (2), 370-383.

5. Wei, J. M.; Iglesia, E., Isotopic and kinetic assessment of the mechanism of methane reforming and decomposition reactions on supported iridium catalysts. Phys. Chem. Chem. Phys. 2004, 6, (13), 3754-3759.

6. Wei, J. M.; Iglesia, E., Structural and mechanistic requirements for methane activation and chemical conversion on supported iridium clusters. Angew. Chem. 2004, 43, (28), 3685-3688.

7. Zhang, X. G.; Liyanage, R.; Armentrout, P. B., Potential energy surface for activation of methane by Pt+: A combined guided ion beam and DFT study. J. Am. Chem. Soc. 2001, 123, (23), 5563-5575.

8. Luntz, A. C.; Bethune, D. S., Activation of Methane Dissociation on a $\operatorname{Pt}(111)$ Surface. $J$. Chem. Phys. 1989, 90, (2), 1274-1280.

9. Harris, J.; Simon, J.; Luntz, A. C.; Mullins, C. B.; Rettner, C. T., Thermally Assisted Tunneling - $\mathrm{CH}_{4}$ Dissociation on Pt(111). Phys. Rev. Lett. 1991, 67, (5), 652-655.

10. Seets, D. C.; Reeves, C. T.; Ferguson, B. A.; Wheeler, M. C.; Mullins, C. B., Dissociative chemisorption of methane on $\operatorname{Ir}(111)$ : Evidence for direct and trapping-mediated mechanisms. $J$. Chem. Phys. 1997, 107, (23), 10229-10241.

11. Jachimowski, T. A.; Hagedorn, C. J.; Weinberg, W. H., Direct and trapping-mediated dissociative chemisorption of methane on $\operatorname{Ir}(111)$. Surf. Sci. 1997, 393, (1-3), 126-134.

12. Brass, S. G.; Ehrlich, G., Dissociative and Molecular Adsorption of Methane on Rhodium. Surf. Sci. 1987, 187, (1), 21-35.

13. Brass, S. G.; Ehrlich, G., Internal Molecular Motions and Activated Chemisorption - Ch4 on Rhodium. Journal of Chemical Physics 1987, 87, (8), 4285-4293.

14. Nave, S.; Tiwari, A. K.; Jackson, B., Methane dissociation and adsorption on Ni(111), Pt(111), Ni(100), Pt(100), and Pt(110)-(1x2): Energetic study. J. Chem. Phys. 2010, 132, (5), 054705 .

15. Mueller, J. E.; van Duin, A. C. T.; Goddard, W. A., Competing, Coverage-Dependent Decomposition Pathways for C2Hy Species on Nickel (111). J. Phys. Chem. C 2010, 114, (47), 20028-20041.

16. Killelea, D. R.; Campbell, V. L.; Shuman, N. S.; Smith, R. R.; Utz, A. L., Surface Temperature Dependence of Methane Activation on Ni(111). J. Phys. Chem. C 2009, 113, (48), 20618-20622.

17. Maroni, P.; Papageorgopoulos, D. C.; Sacchi, M.; Dang, T. T.; Beck, R. D.; Rizzo, T. R., State-resolved gas-surface reactivity of methane in the symmetric $\mathrm{C}-\mathrm{H}$ stretch vibration on Ni(100). Phys. Rev. Lett. 2005, 94, (24), 246104.

18. Abbott, H. L.; Bukoski, A.; Harrison, I., Microcanonical unimolecular rate theory at surfaces. II. Vibrational state resolved dissociative chemisorption of methane on $\mathrm{Ni}(100) . J$. Chem. Phys. 2004, 121, (8), 3792-3810. 
19. Nielsen, B. O.; Luntz, A. C.; Holmblad, P. M.; Chorkendorff, I., Activated Dissociative Chemisorption of Methane on Ni(100) - a Direct Mechanism under Thermal Conditions. Catal. Lett. 1995, 32, (1-2), 15-30.

20. Nave, S.; Jackson, B., Vibrational mode-selective chemistry: Methane dissociation on Ni(100). Phys. Rev. B 2010, 81, (23), 233408.

21. Yoder, B. L.; Bisson, R.; Beck, R. D., Steric Effects in the Chemisorption of Vibrationally Excited Methane on Ni(100). Science 2010, 329, (5991), 553-556.

22. Zhu, Y. A.; Dai, Y. C.; Chen, D.; Yuan, W. K., First-principles calculations of CH4 dissociation on $\mathrm{Ni}(100)$ surface along different reaction pathways. Journal of Molecular Catalysis a-Chemical 2007, 264, (1-2), 299-308.

23. Juurlink, L. B. F.; Smith, R. R.; Killelea, D. R.; Utz, A. L., Comparative study of C-H stretch and bend vibrations in methane activation on $\mathrm{Ni}(100)$ and $\mathrm{Ni}(111)$. Phys. Rev. Lett. 2005, 94, (20), 208303.

24. Schmid, M. P.; Maroni, P.; Beck, R. D.; Rizzo, T. R., Surface reactivity of highly vibrationally excited molecules prepared by pulsed laser excitation: $\mathrm{CH}_{4}\left(2 \mathrm{n}_{3}\right)$ on $\mathrm{Ni}(100) . J$. Chem. Phys. 2002, 117, (19), 8603-8606.

25. Juurlink, L. B. F.; Smith, R. R.; Utz, A. L., The role of rotational excitation in the activated dissociative chemisorption of vibrationally excited methane on $\mathrm{Ni}(100)$. Faraday Discuss. 2000, 117, 147-160.

26. Bengaard, H. S.; Alstrup, I.; Chorkendorff, I.; Ullmann, S.; Rostrup-Nielsen, J. R.; Norskov, J. K., Chemisorption of methane on $\mathrm{Ni}(100)$ and $\mathrm{Ni}(111)$ surfaces with preadsorbed potassium. J. Catal. 1999, 187, (1), 238-244.

27. Juurlink, L. B. F.; McCabe, P. R.; Smith, R. R.; DiCologero, C. L.; Utz, A. L., Eigenstate-resolved studies of gas-surface reactivity: $\mathrm{CH}_{4}\left(1 \mathrm{n}_{3}\right)$ dissociation on $\mathrm{Ni}(100)$. Phys. Rev. Lett. 1999, 83, (4), 868-871.

28. Holmblad, P. M.; Wambach, J.; Chorkendorff, I., Molecular-Beam Study of Dissociative Sticking of Methane on Ni(100). J. Chem. Phys. 1995, 102, (20), 8255-8263.

29. Dianat, A.; Seriani, N.; Ciacchi, L. C.; Pompe, W.; Cuniberti, G.; Bobeth, M., Dissociative Adsorption of Methane on Surface Oxide Structures of Pd-Pt Alloys. J. Phys. Chem. C 2009, 113, (50), 21097-21105.

30. Tait, S. L.; Dohnalek, Z.; Campbell, C. T.; Kay, B. D., Methane adsorption and dissociation and oxygen adsorption and reaction with $\mathrm{CO}$ on $\mathrm{Pd}$ nanoparticles on $\mathrm{MgO}(100)$ and on Pd(111). Surf. Sci. 2005, 591, (1-3), 90-107.

31. Kao, C. L.; Madix, R. J., The adsorption dynamics of molecular methane, propane, and neopentane on Pd(111): Theory and experiment. J. Phys. Chem. B 2002, 106, (33), 8248-8257.

32. Egeberg, R. C.; Ullmann, S.; Alstrup, I.; Mullins, C. B.; Chorkendorff, I., Dissociation of CH4 on Ni(111) and Ru(0001). Surf Sci 2002, 497, (1-3), 183-193.

33. Wu, M.-C.; Goodman, D. W., High-resolution electron energy-loss measurements of sticking coefficients of methane decomposition on Ru(0001). Surf. Sci. 1994, 306, (1-2), L529.

34. Larsen, J. H.; Holmblad, P. M.; Chorkendorff, I., Dissociative sticking of $\mathrm{CH} 4$ on Ru(0001). J. Chem. Phys. 1999, 110, (5), 2637-2642.

35. Mortensen, H.; Diekhoner, L.; Baurichter, A.; Luntz, A. C., CH4 dissociation on $\mathrm{Ru}(0001)$ : A view from both sides of the barrier. J. Chem. Phys. 2002, 116, (13), 5781-5794.

36. Schoofs, G. R.; Arumainayagam, C. R.; Mcmaster, M. C.; Madix, R. J., Dissociative Chemisorption of Methane on Pt(111). Surf. Sci. 1989, 215, (1-2), 1-28.

37. Ukraintsev, V. A.; Harrison, I., A Statistical-Model for Activated Dissociative Adsorption - Application to Methane Dissociation on Pt(111). J. Chem. Phys. 1994, 101, (2), 1564-1581. 
38. Bukoski, A.; Blumling, D.; Harrison, I., Microcanonical unimolecular rate theory at surfaces. I. Dissociative chemisorption of methane on $\operatorname{Pt}(111)$. J. Chem. Phys. 2003, 118, (2), 843-871.

39. DeWitt, K. M.; Valadez, L.; Abbott, H. L.; Kolasinski, K. W.; Harrison, I., Using effusive molecular beams and microcanonical unimolecular rate theory to characterize $\mathrm{CH}_{4}$ dissociation on Pt(111). J. Phys. Chem. B 2006, 110, (13), 6705-6713.

40. Cushing, G. W.; Navin, J. K.; Donald, S. B.; Valadez, L.; Johanek, V.; Harrison, I., C-H Bond Activation of Light Alkanes on Pt(111): Dissociative Sticking Coefficients, Evans-Polanyi Relation, and Gas-Surface Energy Transfer. J. Phys. Chem. C 2010, 114, (40), 17222-17232.

41. Cushing, G. W.; Navin, J. K.; Donald, S. B.; Valadez, L.; Johánek, V.; Harrison, I., Addition/Correction to: C-H Bond Activation of Light Alkanes on Pt(111): Dissociative Sticking Coefficients, Evans-Polanyi Relation, and Gas-Surface Energy Transfer (vol 114, pg 17222, 2010). J. Phys. Chem. C 2010, 114, (51), 22790-22790.

42. $\quad$ Egeberg, R. C.; Ullmann, S.; Alstrup, I.; Mullins, C. B.; Chorkendoff, I., Dissociation of $\mathrm{CH}_{4}$ on $\mathrm{Ni}(111)$ and $\mathrm{Ru}(0001)$. Surf. Sci. 2002, 497, (1-3), 183-193.

43. Alstrup, I.; Chorkendorff, I.; Ullmann, S., Dissociative Chemisorption of CH4 on Ni(100) with Preadsorbed Oxygen. Surf. Sci. 1990, 234, (1-2), 79-86.

44. Sault, A. G.; Goodman, D. W., Dissociative adsorption of alkanes on Ni(100) comparison with molecular-beam results. J. Chem. Phys. 1988, 88, (11), 7232-7239.

45. Beebe, T. P.; Goodman, D. W.; Kay, B. D.; Yates, J. T., Kinetics of the Activated Dissociative Adsorption of Methane on the Low Index Planes of Nickel Single-Crystal Surfaces. J. Chem. Phys. 1987, 87, (4), 2305-2315.

46. Anderson, A. B.; Maloney, J. J., Activation of Methane on Iron, Nickel, and Platinum Surfaces - a Molecular-Orbital Study. J. Phys. Chem. 1988, 92, (3), 809-812.

47. Liao, M. S.; Au, C. T.; Ng, C. F., Methane dissociation on Ni, Pd, Pt and Cu metal(111) surfaces - A theoretical comparative study. Chem. Phys. Lett. 1997, 272, (5-6), 445-452.

48. Shustorovich, E.; Sellers, H., The UBI-QEP method: a practical theoretical approach to understanding chemistry on transition metal surfaces. Surf. Sci. Rep. 1998, 31, (1-3), 5-119.

49. Au, C. T.; Ng, C. F.; Liao, M. S., Methane dissociation and syngas formation on Ru, Os, Rh, Ir, Pd, Pt, Cu, Ag, and Au: A theoretical study. J. Catal. 1999, 185, (1), 12-22.

50. Michaelides, A.; Hu, P., Insight into microscopic reaction pathways in heterogeneous catalysis. J. Am. Chem. Soc. 2000, 122, (40), 9866-9867.

51. Psofogiannakis, G.; St-Amant, A.; Ternan, M., Methane oxidation mechanism on Pt(111): A cluster model DFT study. J. Phys. Chem. B 2006, 110, (48), 24593-24605.

52. Vines, F.; Lykhach, Y.; Staudt, T.; Lorenz, M. P. A.; Papp, C.; Steinruck, H. P.; Libuda, J.; Neyman, K. M.; Gorling, A., Methane Activation by Platinum: Critical Role of Edge and Corner Sites of Metal Nanoparticles. Chemistry-a European Journal 2010, 16, (22), 6530-6539.

53. Liu, Z. P.; Hu, P., General rules for predicting where a catalytic reaction should occur on metal surfaces: A density functional theory study of $\mathrm{C}-\mathrm{H}$ and $\mathrm{C}-\mathrm{O}$ bond breaking/making on flat, stepped, and kinked metal surfaces. J. Am. Chem. Soc. 2003, 125, (7), 1958-1967.

54. Lin, Y. Z.; Sun, J.; Yi, J.; Lin, J. D.; Chen, H. B.; Liao, D. W., Energetics of chemisorption and conversion of methane on transition metal surfaces. Journal of Molecular Structure-Theochem 2002, 587, 63-71.

55. Liu, Z. P.; Hu, P., General trends in the barriers of catalytic reactions on transition metal surfaces. J. Chem. Phys. 2001, 115, (11), 4977-4980.

56. Ciobica, I. M.; Frechard, F.; van Santen, R. A.; Kleyn, A. W.; Hafner, J., A DFT study of transition states for C-H activation on the Ru(0001) surface. J. Phys. Chem. B 2000, 104, (14), 3364-3369.

57. Jackson, B.; Nave, S., The dissociative chemisorption of methane on Ni(100): Reaction path description of mode-selective chemistry. J. Chem. Phys. 2011, 135, (11), 114701. 
58. Yoder, B. L.; Bisson, R.; Hundt, P. M.; Beck, R. D., Alignment dependent chemisorption of vibrationally excited $\mathrm{CH}_{4}\left(\mathrm{n}_{3}\right)$ on $\mathrm{Ni}(100), \mathrm{Ni}(110)$, and $\mathrm{Ni}(111)$. J. Chem. Phys. 2011, 135, (22), 224703.

59. Nave, S.; Jackson, B., Methane dissociation on $\mathrm{Ni}(111)$ and $\mathrm{Pt}(111)$ : Energetic and dynamical studies. J. Chem. Phys. 2009, 130, (5), 054701.

60. Bengaard, H. S.; Norskov, J. K.; Sehested, J.; Clausen, B. S.; Nielsen, L. P.; Molenbroek, A. M.; Rostrup-Nielsen, J. R., Steam reforming and graphite formation on Ni catalysts. J. Catal. 2002, 209, (2), 365-384.

61. Kratzer, P.; Hammer, B.; Norskov, J. K., A theoretical study of CH4 dissociation on pure and gold-alloyed Ni(111) surfaces. J. Chem. Phys. 1996, 105, (13), 5595-5604.

62. Nave, S.; Jackson, B., Methane dissociation on Ni(111): The role of lattice reconstruction. Phys. Rev. Lett. 2007, 98, (17), 173003.

63. Bisson, R.; Sacchi, M.; Dang, T. T.; Yoder, B.; Maroni, P.; Beck, R. D., State-resolved reactivity of $\mathrm{CH}_{4}\left(2 \mathrm{n}_{3}\right)$ on $\mathrm{Pt}(111)$ and $\mathrm{Ni}(111)$ : Effects of barrier height and transition state location. J. Phys. Chem. A 2007, 111, (49), 12679-12683.

64. Abild-Pedersen, F.; Lytken, O.; Engbaek, J.; Nielsen, G.; Chorkendorff, I.; Norskov, J. K., Methane activation on Ni(111): Effects of poisons and step defects. Surf. Sci. 2005, 590, (23), 127-137.

65. Carré, M. N.; Jackson, B., Dissociative chemisorption of $\mathrm{CH}_{4}$ on Ni: The role of molecular orientation. J. Chem. Phys. 1998, 108, (9), 3722-3730.

66. Akinaga, Y.; Taketsugu, T.; Hirao, K., Theoretical study of $\mathrm{CH} 4$ photodissociation on Pd and Ni(111) surfaces. J. Chem. Phys. 1998, 109, (24), 11010-11017.

67. Yang, H.; Whitten, J. L., Dissociative Chemisorption of CH4 on Ni(111). J. Chem. Phys. 1992, 96, (7), 5529-5537.

68. Hanley, L.; Xu, Z.; Yates, J. T., Methane Activation on Ni(111) at High-Pressures. Surf. Sci. 1991, 248, (3), L265-L273.

69. Ceyer, S. T., Translational and Collision-Induced Activation of Ch4 on $\mathrm{Ni}(111)-$ Phenomena Connecting Ultra-High-Vacuum Surface Science to High-Pressure Heterogeneous Catalysis. Langmuir 1990, 6, (1), 82-87.

70. Yang, Q. Y.; Johnson, A. D.; Maynard, K. J.; Ceyer, S. T., Synthesis of Benzene from Methane over a Ni(111) Catalyst. J. Am. Chem. Soc. 1989, 111, (23), 8748-8749.

71. Beckerle, J. D.; Johnson, A. D.; Yang, Q. Y.; Ceyer, S. T., Collision-Induced Dissociation and Desorption - Ch4 and Co on Ni(111). J. Vac. Sci. Technol. A 1988, 6, (3), $903-$ 904.

72. Larsen, J. H.; Chorkendorff, I., From fundamental studies of reactivity on single crystals to the design of catalysts. Surf. Sci. Rep. 1999, 35, (5-8), 165-222.

73. Winters, H. F., The kinetic isotope effect in the dissociative chemisorption of methane. Journal of Chemical Physics 1976, 64, 3495-3500.

74. Rettner, C. T.; Pfnur, H. E.; Auerbach, D. J., Dissociative Chemisorption of CH4 on W(110) - Dramatic Activation by Initial Kinetic-Energy. Phys. Rev. Lett. 1985, 54, (25), 27162719 .

75. Lo, T. C.; Ehrlich, G., Activated Chemisorption of Methane and Tunneling - a 2nd Look - Reply. Surf. Sci. 1988, 198, (3), L380-L385.

76. Lo, T. C.; Ehrlich, G., Activated Chemisorption of Methane and Tunneling. Surf. Sci. 1987, 179, (1), L19-L25.

77. Lee, M. B.; Yang, Q. Y.; Ceyer, S. T., Dynamics of the Activated Dissociative Chemisorption of $\mathrm{CH} 4$ and Implication for the Pressure Gap in Catalysis - a Molecular-Beam High-Resolution Electron-Energy Loss Study. J. Chem. Phys. 1987, 87, (5), 2724-2741.

78. Ceyer, S. T., New Mechanisms for Chemistry at Surfaces. Science 1990, 249, (4965), 133-139. 
79. Luntz, A. C., CH4 Dissociation on Ni(100) - Comparison of a Direct Dynamical Model to Molecular-Beam Experiments. J. Chem. Phys. 1995, 102, (20), 8264-8269.

80. Abbott, H. L.; Bukoski, A.; Kavulak, D. F.; Harrison, I., Dissociative chemisorption of methane on $\mathrm{Ni}(100)$ : Threshold energy from $\mathrm{CH}_{4} \quad\left(2 \mathrm{n}_{3}\right)$ eigenstate-resolved sticking measurements. J. Chem. Phys. 2003, 119, (13), 6407-6410.

81. Yoder, B. L.; Bisson, R.; Hundt, P. M.; Beck, R. D., Alignment dependent chemisorption of vibrationally excited $\mathrm{CH} 4(\mathrm{nu}(3))$ on $\mathrm{Ni}(100), \mathrm{Ni}(110)$, and Ni(111). J. Chem. Phys. 2011, 135, (22), 224703.

82. Luntz, A. C., A simple model for associative desorption and dissociative chemisorption. J. Chem. Phys. 2000, 113, (16), 6901-6905.

83. Swang, O.; Faegri, K.; Gropen, O.; Wahlgren, U.; Siegbahn, P., A Theoretical-Study of the Chemisorption of Methane on a Ni(100) Surface. Chem. Phys. 1991, 156, (3), 379-386.

84. Milot, R.; Jansen, A. P. J., Bond breaking in vibrationally excited methane on transitionmetal catalysts. Phys. Rev. B 2000, 61, (23), 15657-15660.

85. Halonen, L.; Bernasek, S. L.; Nesbitt, D. J., Reactivity of vibrationally excited methane on nickel surfaces. J. Chem. Phys. 2001, 115, (12), 5611-5619.

86. Bukoski, A.; Abbott, H. L.; Harrison, I., Microcanonical unimolecular rate theory at surfaces. III. Thermal dissociative chemisorption of methane on $\operatorname{Pt}(111)$ and detailed balance. $J$. Chem. Phys. 2005, 123, (9), 094707.

87. Abbott, H. L.; Bukoski, A.; Kavulak, D. F.; Harrison, I., Dissociative chemisorption of methane on $\mathrm{Ni}(100)$ : Threshold energy from $\mathrm{CH}_{4}\left(2 \mathrm{n}_{3}\right)$ eigenstate-resolved sticking measurements. Journal of Chemical Physics 2003, 119, 6407-6411.

88. Dahl, S.; Logadottir, A.; Egeberg, R. C.; Larsen, J. H.; Chorkendorff, I.; Tornqvist, E.; Norskov, J. K., Role of steps in N2 activation on Ru(0001). Phys. Rev. Lett. 1999, 83, (9), 18141817.

89. Zambelli, T.; Wintterlin, J.; Trost, J.; Ertl, G., Identification of the "active sites" of a surface-catalyzed reaction. Science 1996, 273, (5282), 1688-1690.

90. Carstens, J. N.; Bell, A. T., Methane activation and conversion to higher hydrocarbons on supported ruthenium. J Catal 1996, 161, (1), 423-429.

91. Wei, J. M.; Iglesia, E., Reaction Pathways and Site Requirements for the Activation and Chemical Conversion of Methane on Ru-Based Catalysts. J. Phys. Chem. B 2004, 108, 72537262.

92. Wei, J. M.; Iglesia, E., Isotopic and kinetic assessment of the mechanism of reactions of $\mathrm{CH} 4$ with $\mathrm{CO} 2$ and $\mathrm{H} 2 \mathrm{O}$ to form synthesis gas and carbon on nickel catalysts. J. Catal. 2004, 224, 370-383.

93. Wei, J. M.; Iglesia, E., Structural requirements and reaction pathways in methane activation and chemical conversion catalyzed by rhodium. J. Catal. 2004, 225, 116-127.

94. Wei, J. M.; Iglesia, E., Structural and Mechanistic Requirements for Methane Activation and Chemical Conversion on Supported Iridium Clusters. Angew. Chem. Int. Ed. 2004, 43, 36853688 .

95. Henkelman, G.; Jonsson, H., Theoretical calculations of dissociative adsorption of $\mathrm{CH}_{4}$ on an $\operatorname{Ir}(111)$ surface. Phys. Rev. Lett. 2001, 86, (4), 664-667.

96. Donald, S. B.; Harrison, I., Dynamically biased RRKM model of activated gas-surface reactivity: vibrational efficacy and rotation as a spectator in the dissociative chemisorption of $\mathrm{CH}_{4}$ on Pt(111). Phys. Chem. Chem. Phys. 2012, 14, (5), 1784-1796.

97. Baer, T.; Mayer, P. M., Statistical Rice-Ramsperger-Kassel-Marcus quasiequilibrium theory calculations in mass spectrometry. J. Am. Soc. Mass. Spectrom. 1997, 8, (2), 103-115.

98. Baer, T.; Hase, W. L., Unimolecular Reaction Dynamics. Oxford University Press: New York, NY, 1996. 
99. Murphy, M. J.; Hodgson, A., Adsorption and desorption dynamics of $\mathrm{H}_{2}$ and $\mathrm{D}_{2}$ on $\mathrm{Cu}(111)$ : The role of surface temperature and evidence for corrugation of the dissociation barrier. J. Chem. Phys. 1998, 108, (10), 4199-4211.

100. Tolman, R. C., Statistical merchanics applied to chemical kinetics. J. Am. Chem. Soc. 1920, 42, (12), 2506-2528.

101. Juurlink, L. B. F.; Killelea, D. R.; Utz, A. L., State-resolved probes of methane dissociation dynamics. Prog. Surf. Sci. 2009, 84, (3-4), 69-134.

102. Abbott, H. L.; Harrison, I., Dissociative chemisorption and energy transfer for methane on $\operatorname{Ir}(111)$. J. Phys. Chem. B 2005, 109, (20), 10371-10380.

103. Luntz, A. C.; Winters, H. F., Dissociation of Methane and Ethane on Pt(110) - Evidence for a Direct Mechanism under Thermal Conditions. J. Chem. Phys. 1994, 101, (12), 1098010989.

104. DeWitt, K. M.; Valadez, L.; Abbott, H. L.; Kolasinski, K. W.; Harrison, I., Effusive molecular beam study of $\mathrm{C}_{2} \mathrm{H}_{6}$ dissociation on Pt(111). J. Phys. Chem. B 2006, 110, (13), 67146720 .

105. DeWitt, K. M.; Valadez, L.; Abbott, H. L.; Kolasinski, K. W.; Harrison, I., Using Effusive Molecular Beams and Microcanonical Unimolecular Rate Theory to Characterize $\mathrm{CH}_{4}$ Dissociation on $\mathrm{Pt}(111)$. J. Phys. Chem. B 2006, 110, in press.

106. Cramer, C. J.; Truhlar, D. G., Density functional theory for transition metals and transition metal chemistry. Phys. Chem. Chem. Phys. 2009, 11, (46), 10757-10816.

107. Rozanska, X.; Sauer, J., Oxidative conversion of C1-C3 alkanes by vanadium oxide catalysts. DFT results and their accuracy. Int. J. Quantum Chem 2008, 108, (12), 2223-2229.

108. Miller, W. H., Unified statistical-model for complex and direct reaction-mechanisms. $J$. Chem. Phys. 1976, 65, (6), 2216-2223.

109. Diaz, C.; Olsen, R. A., A note on the vibrational efficacy in molecule-surface reactions. $J$. Chem. Phys. 2009, 130, (9), 094706.

110. Lee, M. B.; Yang, Q. Y.; Tang, S. L.; Ceyer, S. T., Activated Dissociative Chemisorption of $\mathrm{CH} 4$ on $\mathrm{Ni}(111)$ - Observation of a Methyl Radical and Implication for the Pressure Gap in Catalysis. J. Chem. Phys. 1986, 85, (3), 1693-1694.

111. Smith, R. R.; Killelea, D. R.; DelSesto, D. F.; Utz, A. L., Preference for vibrational over translational energy in a gas-surface reaction. Science 2004, 304, (5673), 992-995.

112. Higgins, J.; Conjusteau, A.; Scoles, G.; Bernasek, S. L., State selective vibrational $\left(2 \mathrm{n}_{3}\right)$ activation of the chemisorption of methane on Pt (111). J. Chem. Phys. 2001, 114, (12), 52775283.

113. Utz, A. L., Mode selective chemistry at surfaces. Current Opinion in Solid State \& Materials Science 2009, 13, (1-2), 4-12. 


\title{
5.
}

\section{RRKM Simulation of Small Alkane Dissociation on Pt(111):}

\section{Addressing Energy Transfer with a Master Equation}

\begin{abstract}
A precursor mediated microcanonical trapping model of activated dissociative chemisorption was used to analyze effusive molecular beam measurements of dissociative sticking coefficients, $S_{n}\left(T_{g}, T_{s}\right)$, for light alkanes incident along the direction of the surface normal, as a function of gas and surface temperatures. Explicit accounting of the gas-surface energy transfer for nonequilibrium experiments became increasingly important as the alkane size was increased. A simple exponential down model of the molecule/phonon collision step size distribution with a mean energy down parameter of $\alpha=700 \mathrm{~cm}^{-1}$ for ethane and $1400 \mathrm{~cm}^{-1}$ for propane sufficed to provide a good description of the $S_{n}\left(T_{g}, T_{s}\right)$ data. Activation energies determined from $S_{n}(T)$ modeling for methane, ethane, and propane are $E_{a}=59 \mathrm{~kJ} \mathrm{~mol}^{-1}, 44 \mathrm{~kJ} \mathrm{~mol}^{-1}$, and $30 \mathrm{~kJ} \mathrm{~mol}^{-1}$, respectively, which compare to experimental values of $E_{a}=58 \mathrm{~kJ} \mathrm{~mol}^{-1}, 43 \mathrm{~kJ} \mathrm{~mol}^{-1}$, and $34 \mathrm{~kJ}$ $\mathrm{mol}^{-1}$. An Evans-Polanyi plot of $E_{a}$ for alkane dissociative chemisorption evaluated at $\mathrm{T}=600 \mathrm{~K}$ versus the alkane thermal desorption energy, $E_{d}$, is linear with a slope of -0.608. Assuming that the alkane $E_{d}$ serves as a good approximation to the van der Waals stabilization of the chemisorbed products of dissociative chemisorption, the slope of the Evans-Polanyi plot indicates a slightly late state transition barrier for alkane dissociative chemisorption on $\operatorname{Pt}(111)$ with respect to van der Waals binding to the surface.
\end{abstract}




\subsection{Introduction}

As the demand on fossil fuel reserves continues to increase, the efficient utilization of the light alkanes comprising the primary component will become increasingly important they provide some of the essential feedstocks for the chemical industry. Currently, these small hydrocarbons are often converted to synthesis gas either through steam reforming or catalytic partial oxidation on transition metal nanocatalysts. Alternatively, the individual alkane components can be separated and catalytically converted into other industrially useful compounds (e.g. ethane to ethylene, propane to acrylic acid, etc). $\mathrm{C}-\mathrm{H}$ bond activation of alkanes has remained a topic of considerable practical and fundamental interest ${ }^{1}$ because forging a microscopic level understanding of the mechanism of alkane/surface reactions may ultimately prove useful for optimizing catalysts and catalytic processes for energy efficiency and product selectivity.

Although activated dissociative chemisorption of molecules at surfaces is often considered to be the rate determining step in heterogeneous catalytic processes, ${ }^{2}$ there is only a modest quantity of experimental information available concerning alkane dissociative sticking. ${ }^{3}$ Kinetics studies by Wei and Igelsia ${ }^{4,5}$ have shown that methane reforming over supported metal nanocatalysts under pseudo-catalytic conditions (i.e. temperatures near $873 \mathrm{~K}$ and 1 bar pressure), is rate limited by cleavage of the initial $\mathrm{C}-\mathrm{H}$ bond during methane dissociative chemisorption. Surprisingly, while methane reforming has been shown to be a structure sensitive process, ${ }^{4,5}$ surface science studies find that thermal dissociative sticking coefficients for methane on flat metal surfaces are several orders of magnitude higher than values derived from reforming turnover rates on metal nanocatalysts. ${ }^{6-8}$

To measure extremely low dissociative sticking coefficients, such as those for methane on Ni surfaces, intermediate pressure "thermal bulb" techniques were developed by Yates and 
Goodman, ${ }^{9}$ and later extended by Chorkendorff. ${ }^{6}$ Heating a surface in an ambient gas at a pressure of several Torr ${ }^{6}$ can permit collisional energy transfer to thermalize the gas layer within a mean free path of the surface to the surface temperature such that a thermal dissociative sticking coefficient can be measured. For a single pure gas, the pressures required for thermalization coupled with a gas-surface collision flux of $\sim 10^{6}$ monolayers $\mathrm{s}^{-1}$ Torr $^{-1}$ tends to make the thermal bulb technique best suited for measurements of thermal dissociative sticking coefficients less than $S(T) \sim 10^{-6}$ because otherwise the dosing times become overly short. However, if the reactive gas is diluted in an inert buffer gas, higher values of $S(T)$ can be measured ${ }^{10}$ while maintaining the several Torr net pressure typically required for local gas/surface thermalization. ${ }^{6}$ Alternatively, heated effusive molecular beams have been used to prepare small alkanes with easily characterizable thermal energy distributions, which can be sent towards a single crystal metal surface along the direction of the surface normal. Integration of angular distributions measured from an effusive beam technique allows for calculation of thermal dissociative sticking coefficients of an ambient gas, easily relatable to a catalysis regime.

Supersonic molecular beams provide an important means to investigate non-equilibrium dissociative sticking coefficients as a function of a molecule's translational energy and internal energy, and the surface temperature. ${ }^{3,11-14}$ With the aid of laser-pumped supersonic molecular beams, rovibrational eigenstate-resolved dissociative sticking coefficients for methane ${ }^{15-17}$ and silane ${ }^{18}$ have begun to be measured. The reactivity of methane on a variety of metal surfaces has not been found to be purely statistical. Vibrational efficacies for reaction relative to normal translational energy have been determined to vary over the range $0.4 \leq \eta_{v} \leq 1.4$, with an experimental average to date of $\left\langle\eta_{v}\right\rangle=0.88 .{ }^{19}$ In the previous two chapters, the d-PMMT has been applied to the dissociative chemisorption of methane on $\operatorname{Ir}(111), \mathrm{Ni}(100), \mathrm{Ni}(111)$, 
$\operatorname{Ru}(0001)$, and $\operatorname{Pt}(111)$ with values of $\eta_{v}$ varying from 0.05 to 0.72 . The value of $\eta_{v}$ appropriate to statistical transition state theories of chemical reactivity is $\eta_{v}=1$.

In this chapter we apply a master equation precursor mediated microcanonical trapping (ME-PMMT) model of gas surface reactivity that includes energy exchange between the physisorbed precursor complexes and surrounding surface bulk to analyze or predict dissociative sticking coefficients derived from effusive or supersonic molecular beam experiments.

DFT calculations for $\mathrm{C}_{2} \mathrm{H}_{6}$ dissociative chemisorption on Pt surfaces find $E_{0}=52 \mathrm{~kJ} / \mathrm{mol}$ for $\operatorname{Pt}(111),{ }^{20}$ and two transition states for $\operatorname{Pt}(110)$ with $E_{0}=37 \mathrm{~kJ}$ and $41 \mathrm{~kJ} / \mathrm{mol} .{ }^{21}$ Previous PMMT analysis of ethane dissociative chemisorption on $\operatorname{Pt}(111)$ effusive beam experiments ${ }^{22}$ gave a threshold barrier to dissociative of $E_{0}=34 \mathrm{~kJ} / \mathrm{mol}$. A thermal bulb study ${ }^{23}$ performed over the temperature range $515 \leq S(T) \leq 635 K$ measured ambient dissociative thermal sticking coefficients, $S(T)$, roughly 130 -fold smaller than the $S_{n}(T)$ values measured using effusive beam techniques at $T=600 \mathrm{~K}^{22}$ The activation energy for the thermal bulb $S(T)$ was $E_{a}=37 \pm 3 \mathrm{~kJ} / \mathrm{mol}$, while effusive beam experiments led to activation energies of $E_{a}=44.3^{22}$ and $42.7 \mathrm{~kJ} / \mathrm{mol}^{24,25}$. A recent GGA-DFT study of propane dissociative chemisorption on $\mathrm{Pt}_{4}$ subnanometer catalysts and on $\operatorname{Pt}(111)^{26}$ calculated threshold energies for dissociative chemisorption of $E_{0}=17 \mathrm{~kJ}$ for the $\mathrm{Pt}_{4}$ clusters and $E_{0}=114 \mathrm{~kJ} / \mathrm{mol}$ for $\mathrm{Pt}(111)$. PMMT analysis of effusive beam experiments found $E_{0}=23.6 \mathrm{~kJ} / \mathrm{mol}$, nearly an order of magnitude smaller than the DFT prediction for $\operatorname{Pt}(111)$ (n.b. $\mathrm{E}_{0}$ is exponentially amplified in the rate equiation!).

\subsection{ME-PMMT Model}


A precursor mediated microcanonical trapping model of activated dissociative chemisorption has been described previously; its application to alkanes, $\mathrm{C}_{\mathrm{n}} \mathrm{H}_{2 \mathrm{n}+2}$, is schematically depicted in Figure 5.1. The kinetic formalism for the ME-PMMT model is given in Eq. 5.1,

$$
\begin{aligned}
& R H_{(p)}\left(E^{\prime}\right) \\
& R\left(E, E^{\prime}\right) \| \uparrow R\left(E^{\prime}, E\right) \\
& R H_{(g)} \underset{k_{D}(E)}{\stackrel{F_{o} f(E)}{\rightleftarrows}} R H_{(p)}(E) \stackrel{k_{R}(E)}{\longrightarrow} R_{(c)}+H_{(c)}
\end{aligned}
$$

such that the ME for the PC coverage distribution is,

$$
\begin{aligned}
\frac{d \theta_{p}(E)}{d t} d E= & F_{o} f(E)-\left\{k_{R}(E)+k_{D}(E)\right\} \theta_{p}(E) d E \\
& +\int_{0}^{\infty}\left\{R\left(E, E^{\prime}\right) \theta_{p}\left(E^{\prime}\right) d E-R\left(E^{\prime}, E\right) \theta_{p}(E) d E^{\prime}\right\}
\end{aligned}
$$

where $F_{o}$ is the incoming molecular flux in ML/s, $f(E)$ is the flux distribution for forming a PC at energy $\mathrm{E}$, the $k_{i}$ are the RRKM rate constants to desorb or react, and $R\left(E, E^{\prime}\right)$ is the rate distribution for $\mathrm{PC} /$ metal energy transfer taking a PC at energy E' to energy E. Typically, in gas phase energy transfer involving bimolecular collisions, the $R\left(E, E^{\prime}\right)$ energy transfer rate constants are written as the product of an inelastic collision frequency, $\omega$, and a collision step size distribution, $P\left(E, E^{\prime}\right)$, so that $R\left(E, E^{\prime}\right)=\omega P\left(E, E^{\prime}\right) .{ }^{27}$ The collision frequency for the PCs and surrounding substrate was taken to be three times the mean phonon frequency of the bulk metal because the PCs are bathed by phonons incoherently from three directions. The alkane MEPMMT calculations employed a simple exponential down model of the PC/phonon collision step size distribution described for downwards energy transfer as, 


$$
P\left(E, E^{\prime}\right)=\frac{1}{N\left(E^{\prime}\right)} \exp \left\{-\frac{E^{\prime}-E}{\alpha}\right\} \quad E^{\prime}-E>0
$$

where $N\left(E^{\prime}\right)$ is a normalization constant and $\alpha$ is related to the mean energy transferred in a downwards collision ${ }^{28}$ by,

$\alpha \approx\left\langle\Delta E\left(E^{\prime}\right)\right\rangle_{\text {down }}=\frac{\int_{0}^{E^{\prime}}\left(E^{\prime}-E\right) P\left(E, E^{\prime}\right) d E}{\int_{0}^{E^{\prime}} P\left(E, E^{\prime}\right) d E}$

when $\alpha \leq k_{b} T$. The upwards energy transfer is fixed by the detailed balance requirements that the net energy transfer should be zero under thermal equilibrium conditions, which would be reached if the desorption and reaction channels were turned off. The detailed balance requirement is simply that the bracketed term within the integral of Eq. (5.2) must be zero for a thermal precursor complex distribution. With the $R\left(E, E^{\prime}\right)=\omega P\left(E, E^{\prime}\right)$ form of the energy exchange, Eq. (5.2) can be written as,

$$
\begin{aligned}
\frac{d \theta_{p}(E, t)}{d t} & =F(E, t)-\left\{k_{R}(E)+k_{D}(E)+\omega\right\} \theta_{p}(E, t) \\
& +\omega \int_{0}^{\infty} P\left(E, E^{\prime}\right) \theta_{p}\left(E^{\prime}, t\right) d E^{\prime}
\end{aligned}
$$

and solved numerically using matrix methods. ${ }^{28,29}$ When a time independent flux of gas is made incident on the surface according to $F(E, t)=F_{0} f(E)$, the steady state approximation applied to the $\theta_{p}(E, t)$ coverage distribution of Eq (5.10) yields the sticking coefficient,

$$
S=\frac{1}{F_{0}} \frac{d \theta_{c}}{d t}=\frac{1}{F_{0}} \int_{0}^{\infty} k_{R}(E) \theta_{p}^{s s}(E) d E
$$


where $\theta_{c}$ is the chemisorbed coverage of either of the dissociation fragments of the Eq. (5.1) kinetics. $\theta_{p}^{s s}$ was found by solving Eq. (5.9) using standard energy graining and matrix methods. A formal solution provides some physical insight,

$$
\theta_{p}^{s s}(E)=\frac{F_{o} f(E)+\omega \int_{0}^{\infty} P\left(E, E^{\prime}\right) \theta_{p}^{s s}\left(E^{\prime}\right) d E^{\prime}}{k_{R}(E)+k_{D}(E)+\omega}
$$

where it can be seen that $\theta_{p}^{s s}(E)$ is increased by the incident gas flux and energy transfer from PCs at other energies, and decays via reaction to the chemisorbed products, desorption to the gas phase, and energy transfer to PCs at other energies. In the limit that energy exchange between the surrounding surface bulk and precursor complexes goes to zero (i.e. $\omega \rightarrow 0$ ), substitution of Eq. (5.7) into Eq. (5.6) yields,

$$
S=\int_{0}^{\infty} \frac{k_{R}(E)}{k_{R}(E)+k_{D}(E)} f(E) d E=\int_{0}^{\infty} S(E) f(E) d E,
$$

which defines the microcanonical sticking coefficient, $S(E)$, and the central equation of the sPMMT model. Alternatively, as $\omega \rightarrow 0$, Eq. (5.5) simplifies to

$\frac{d \theta_{p}(E, t)}{d t}=F(E, t)-\left\{k_{R}(E)+k_{D}(E)\right\} \theta_{p}(E, t)$

appropriate to the s-PMMT kinetics,

$$
C_{n} H_{2 n+2(g)} \underset{k_{D}(E)}{\stackrel{F(E)}{\rightleftarrows}} C_{n} H_{2 n+2(p)} \stackrel{k_{R}(E)}{\longrightarrow} C_{n} H_{2 n+1(c)}+H_{(c)} \text {. }
$$


that in the steady state approximation also leads to $S=\int_{0}^{\infty} S(E) f(E) d E$.

In the s-PMMT model, a gas-surface precursor complex (PC), which is comprised of the incident gas molecule and a small number of local surface oscillators, is formed, and within this PC, the exchangeable energy from all degrees of freedom is pooled and microcanonically randomized such that the molecules become transiently trapped in the neighborhood of the physisorption well between the transition states for reaction and desorption. These PCs then go on to either react or desorb with RRKM rate constants,

$$
k_{i}\left(E^{*}\right)=\frac{W_{i}^{\dagger}\left(E-E_{i}\right)}{h \rho(E)}
$$

where $W_{i}^{\dagger}\left(E-E_{i}\right)$ is the sum of states for transition state, i, with a threshold energy $E_{i}$ to either react or desorb, $\rho(E)$ is the PC density of states, and $h$ is Planck's constant. On the oftentimes convenient $E^{*}=E-E_{D}$ energy scale, whose zero occurs for infinitely separated reactants, the apparent threshold energy for reaction is $E_{0}$. The steady state approximation applied to the $\mathrm{C}_{\mathrm{n}} \mathrm{H}_{2 \mathrm{n}+2(\mathrm{p})}$ coverage of Eq (5.10) yields the microcanonical dissociative sticking coefficient shown in Eq. (5.8),

$$
S\left(E^{*}\right)=\frac{k_{R}\left(E^{*}\right)}{k_{R}\left(E^{*}\right)+k_{D}\left(E^{*}\right)}=\frac{W_{R}^{\ddagger}\left(E^{*}-E_{0}\right)}{W_{R}^{\ddagger}\left(E^{*}-E_{0}\right)+W_{D}^{\ddagger}\left(E^{*}\right)},
$$

where, $W_{i}^{*}$ is the sum of states for transition state $i, E_{0}$ is the apparent threshold energy for dissociation, and 


$$
\begin{aligned}
f\left(E^{*}\right)=\int_{0}^{E^{*}} f_{t}\left(E_{t}\right) & \int_{0}^{E^{*}-E_{t}} f_{v}\left(E_{v}\right) \int_{0}^{E^{*}-E_{v}-E_{t}} f_{r}\left(E_{r}\right) \\
& \times f_{s}\left(E^{*}-E_{t}-E_{v}-E_{r}\right) d E_{r} d E_{v} d E_{t},
\end{aligned}
$$

is the flux distribution for creating a PC at $E^{*}=E_{t}+E_{v}+E_{r}+E_{s}$. The $f\left(E^{*}\right)$ is formed by convolution over the various incident gas and surface energy distributions.

For reactive systems with a relatively shallow physisorption well and high threshold energy for reaction, desorption lifetimes at reactive energies $\left(E^{*} \geq E_{0}\right)$ are ultrafast and energy exchange between the PC and surrounding bulk metal can be approximated as zero. Ignoring energy transfer between the PC and bulk yields the statistical (s-) PMMT model of dissociative chemisorption. As alkane size grows, RRKM desorption lifetimes at reactive energies increases rapidly due to the increasing depth of the physisorption well, increasing number of vibrational modes, and falling threshold energy for reaction. Consequently, the Master-Equation (ME)PMMT model that explicitly treats vibrational energy exchange between the PCs and bulk was required to replicate the reactivity of higher order alkanes.

Weaver and Madix showed that that the dissociative sticking coefficients for light alkanes on $\operatorname{Pt}(111)$ obey normal translational energy scaling and so translational energy parallel to the surface was taken to be a spectator degree of freedom. Energy associated with all other molecular degrees of freedom and the energy of $s$ surface oscillators was assumed to be active exchangeable energy within the PCs formed. Dissociative sticking calculations require specification of the transition states for desorption and reaction, as well as the energy transfer parameter, $\alpha$. The desorption transition state was taken to occur when the alkane was freely rotating and vibrating in the gas-phase, far from the $s$ surface oscillators of the united PC. The surface oscillators were assumed to vibrate at the mean phonon frequency of the metal [ 
$v_{s}=\left(\frac{3}{4}\right) k_{B} T_{\text {Debye }} / h$ yielding $122 \mathrm{~cm}^{-1}$ for Pt]. The desorption coordinate was taken as the vibrational, ultimately translational, motion, along the surface normal and so that degree of freedom is missing from the desorption transition state (n.b., also missing are the spectator translational motions parallel to the surface). The transition state for dissociative chemisorption was taken to have the same molecular vibrational frequencies as in the gas phase molecule except for one asymmetric $\mathrm{C}-\mathrm{H}$ stretching mode which was chosen as the reaction coordinate. As the molecule approaches the surface its three rotational modes and translation along the surface normal evolve into relatively low frequency frustrated rotations and vibration along the surface normal. In the interest of minimizing parameterization, these four modes are assumed to share the same low frequency, $v_{D}$, at the reactive transition state and also within the PCs. In this way, only 3 parameters are required for PMMT calculations, $\left\{E_{0}, s, v_{D}\right\}$, where $E_{0}$ is the apparent threshold energy for dissociative chemisorption (as in Fig. 5.1). Because alkane desorption energies, $E_{D}$, are available from thermal programmed desorption experiments, ${ }^{30}$ the ME-PMMT calculations required additional specification of only the gas-surface energy transfer parameter, $\alpha$.

Optimization of the 3-4 parameters of the PMMT models to fit the experimental data was performed by minimizing the average relative discrepancy between theoretical simulations of the dissociative sticking coefficients and experimental values. As shown elsewhere (Eq. 82 of Ref. 28), for modest values of the dissociative sticking coefficient under thermal equilibrium conditions, $S(T)<10^{-2}$, an Arrhenius form for $S(T)$ is recovered which should have little to no dependence on the choice of the number of surface oscillators, $s$, and no dependence on $\alpha$ because the net energy transfer between the surface and the PCs must vanish at thermal equilibrium by detailed balance. PMMT simulations to the fairly similar to $S(T)$, isothermal 
effusive molecular beam sticking coefficient, $S_{n}(T)$, for molecules incident along the surface normal with $T=T_{g}=T_{s}$ were found to be relatively insensitive to the choice of $s$ and $\alpha$. Consequently, fitting $S_{n}(T)$ allowed for an initial, fairly robust, optimization of $E_{0}$ and $v_{D}$. Optimization of $s$ and $\alpha$ required further simulation of the non-equilibrium dissociative sticking coefficients $S_{n}\left(T_{g}, T_{s}\right)$ with $T_{g} \neq T_{s}$.

\subsection{Results and Dissussion}

\subsection{A. Methane}

Figure 3 compares d-PMMT $S\left(T_{g}, T_{s}\right)$ for methane on $\operatorname{Pt}(111)$ with effusive beam measurements using the d-PMMT parameters, $\left\{E_{0}=58.9 \mathrm{~kJ} / \mathrm{mol}, s=2, \eta_{v}=0.40\right\}$ developed in Chapter 3, which have been shown to reproduce dissociative sticking coefficients over a wide range of experimental conditions. The effect of gas-surface energy transfer on the $\mathrm{CH}_{4}$ dissociative sticking coefficients was negligible. An ME-PMMT model was optimized for an energy transfer parameter of $\alpha=50 \mathrm{~cm}^{-1}$, but a value of $\alpha=0 \mathrm{~cm}^{-1}$, which yields a s-PMMT model, gave negligibly different results. A dynamically biased ME-PMMT model has not yet been formulated - the difficulty lies in insuring approach to thermal equilibrium in the absence of dissipative channels (i.e., $\mathrm{k}_{\mathrm{d}}$ and $\mathrm{k}_{\mathrm{r}}$ ) while enforcing dynamical constraints.

\subsection{B. Ethane}

For dissociative chemisorption of ethane on Pt (111) a s-PMMT model was optimized primarily to the $S_{n}(T)$ measurements of Cushing $^{9}$ to yield parameters $\left\{E_{0}=34.4 \mathrm{~kJ} / \mathrm{mol}, s=4, v_{D}=145 \mathrm{~cm}^{-1}\right\}$, that gave the $S_{n}\left(T_{g}, T_{s}\right)$ results of Figure 5.3(a) 
and an ARD of 328\%. The s-PMMT predictions in 5.3(a) show a much greater dependence of $S_{n}\left(T_{g}, T_{s}\right)$ on $\mathrm{T}_{\mathrm{g}}$ than experimentally observed. A ME-PMMT model was refined to yield an energy transfer parameter of $\alpha=700 \mathrm{~cm}^{-1}$. ME-PMMT simulations of $S_{n}\left(T_{g}, T_{s}\right)$ are compared to effusive beam measurements in Figure 5.3(b). The ARD between the ME-PMMT and experimental $S_{n}\left(T_{g}, T_{s}\right)$ values decreased to $45 \%$.

\subsection{Propane}

Dissociative sticking coefficients, $S_{n}\left(T_{g}, T_{s}\right)$, measured by Cushing ${ }^{9}$ for propane on $\mathrm{Pt}$ (111) were simulated using the s-PMMT model to yield an optimal parameter set of $\left\{E_{0}=23.6 \mathrm{~kJ} / \mathrm{mol}, s=4, v_{D}=65 \mathrm{~cm}^{-1}\right\}$ with the results shown in 5.4(a), where an ARD of $637 \%$ is achieved. Refinement of a ME-PMMT model led to an energy transfer parameter of $\alpha=1400 \mathrm{~cm}^{-1}$ for propane dissociative sticking. The ME-PMMT simulations of $S_{n}\left(T_{g}, T_{s}\right)$ are compared to effusive beam measurements in Fig. 5.4(b). The ARD between the ME-PMMT and experimental $S_{n}\left(T_{g}, T_{s}\right)$ decreased to $36 \%$. With inclusion of energy transfer in the MEMURT model the normal thermal dissociative sticking coefficients, $\mathrm{S}_{\mathrm{n}}(\mathrm{T})$, more markedly collapse towards the angle-integrated thermal $S(T)$ than was the case for ethane. Parameter optimization might improve agreement with experiments but we were loath to embark on a full four parameter optimization search and so only optimized the barrier height. In Fig. 5.5(a) experimental $S_{n}\left(T_{g}, T_{s}\right)$ are compared to further optimized ME-PMMT model calculations where the barrier height was dropped to $E_{0}=21.2 \mathrm{~kJ} / \mathrm{mol}$. Fig. 5.5 (b) compares s$(\mathrm{ARD}=31 \%)$ and ME-PMMT $(\mathrm{ARD}=18 \%)$ theoretical $\mathrm{S}\left(\mathrm{E}_{\mathrm{n}}\right)$ against experimental supersonic beam data taken by Madix. ${ }^{31}$ The inclusion of energy transfer decreases $S\left(E_{n}\right)$ for the hyper- 
thermal, non-equilibrium, high translational energy supersonic beam experiments even though the PC lifetime on the surface (versus desorption) is relatively short (i.e. 1.4 ps at $\mathrm{E}^{*} \sim 100 \mathrm{~kJ} / \mathrm{mol}$ ) at these high energies.

In simulations of the effusive beam $S_{n}\left(T_{g}, T_{s}\right)$ experiments, the s-PMMT model overpredicts the effect that gas temperature should have on the sticking coefficients. When optimization was attempted for the entire data set, instead of just the $S_{n}(T)$ subset, the s-PMMT would consistently maximize the allowed number of surface oscillators. In essence, the parameters of the model adjusted to favor the contribution of the surface, whose temperature is fixed by the surrounding heat reservoir to which energy transfer will push towards, while the gas contribution towards the PC energy was diminished in a relative sense. This tendency was observed in optimization for the dissociative chemisorption of both ethane and propane, but not for methane, on Pt (111).

Some portion of the discrepancy between ME-PMMT calculations of experimental effusive and supersonic beam measurements may be accounted for by vibrational cooling during the supersonic expansion. Molecules in supersonic molecular beams have undergone collisionally-induced rotational and vibrational cooling during their supersonic expansion that can leave each molecular vibrational mode at a different temperature. ${ }^{32}$ For small molecules, such as methane, vibrational cooling is inefficient and the vibrational modes stay close to the nozzle temperature of the molecular beam, although the rotational temperature falls considerably. ${ }^{33}$ Larger alkanes are anticipated to undergo more substantial vibrational and rotational cooling during supersonic expansions which make them, in the absence of detailed hot band spectroscopic characterization that could determine their state distributions and energy content, somewhat ambiguous subjects for readily-interpretable kinetic studies of gas-surface reactivity. In contrast, 
a molecular effusive beam prepares thermal well-defined energy distributions for the gas. For this reason, the effusive beam data was chosen for the PMMT parameter determination. In the absence of specific evidence to the contrary, we continued to use $T_{v}=T_{N}$ and $T_{r}=0.1 T_{N}$ when simulating alkane supersonic beam experiments.

An assumption of the PMMT model is that the four low-frequency, active vibrational modes of the transition state, i.e. the three frustrated rotations and the motion of the adsorbing molecule normal to the surface, are all small and equal. Traditionally in the ME-PMMT, the frustrated rotations, $v_{D, r}$, and translation normal to the surface, $v_{D, t}$, have been fixed to be the same in the precursor complex as those of the chemisorbed molecule. A more realistic approach is to directly extract these frequencies for the PCs from experiments. The vibration of the PC along the surface normal, $v_{D, t}$, have been determined from He scattering from alkanes adsorbed on Pt (111). ${ }^{34}$ The value is $v_{D, t}=70 \mathrm{~cm}^{-1}$ for all $\mathrm{n}$-alkanes from $\mathrm{n}=1$ to 9 . The remaining three low frequency vibrations corresponding to frustrated rotations can be fixed by setting all three frequencies as equal to one another and adjusted to recover the appropriate pre-exponential factor from alkane TPD. ${ }^{30}$ However, there is no corresponding experimental method to determine the frequencies of the reactive complex at the transition state, and currently no electronic structure theory calculations providing transition state frequencies have been performed for ethane or propane on $\mathrm{Pt}$ (111). Once transition state frequencies become available from electronic structure theory, they could be incorporated into the PMMT model, removing one of the parameters, $v_{D}$, of the model.

5.3.D Angle-Resolved Thermal Dissociative Sticking Coefficients, $S(T, \vartheta)$ 
PMMT calculations (n.b., s- or d- for methane and s- for ethane and propane) of $S(T, \vartheta)$ for small alkanes on $\operatorname{Pt}(111)$ at $\mathrm{T}=700 \mathrm{~K}$ are shown in Figure 5.5 with best fit parameters to the functional form $S(T, \vartheta)=A+B \cos ^{n}(\vartheta)$ listed in Table 5.1. Though methane dissociative chemisorption could be well modeled without the offset term, $A=S\left(T, \vartheta \rightarrow 90^{\circ}\right)$, both ethane and propane are predicted to have significant dissociative sticking values at high angles of incidence equivalent to $42 \%$ and $57 \%$ of the $S\left(T=700 K, \vartheta=0^{\circ}\right)$ for s-PMMT calculations. The relatively high value of $S\left(T, \vartheta=90^{\circ}\right) / S\left(T, \vartheta=0^{\circ}\right)$ stems from the relatively low importance of molecular normal translational energy, $E_{n}=E_{t} \cos ^{2}(\vartheta)$, in the assembly of sufficient exchangeable energy for PCs to surmount the energy barrier for reaction. Normal translational energy is the only angle dependent exchangeable energy and with increasing alkane size the availability of vibrational energy increases significantly. The number of vibrational modes, (3N-6), increases from 9 to 18 to 27 in the sequence methane, ethane, and propane. With the inclusion of PC/substrate energy transfer, normal translational energy is predicted to have even less impact on the dissociative sticking for ethane and propane, with $S\left(700 K, \vartheta=90^{\circ}\right) / S\left(700 K, \vartheta=0^{\circ}\right) \approx 0.80$.

\subsection{E. Evans-Polanyi Relation}

Figure 5.6 is a plot of the experimental $S_{n}(T)$-derived thermal activation energy, $E_{a}$, for dissociative chemisorption versus the desorption energy, $E_{D},{ }^{30}$ of the light alkanes on $\operatorname{Pt}(111)$. The plot is linear with a slope of -0.60 . Figure 5.6 also provides some extrapolated estimates of $E_{a}$ for larger n-alkanes with known desorption energies. Tait et al. ${ }^{30}$ measured desorption energies, $E_{\text {des }}=13.2 n+2.2 \mathrm{~kJ} / \mathrm{mol}$, for a series of n-alkanes on $\operatorname{Pt}(111)$ and found that octane 
was the smallest $n$-alkane for which some dissociative chemisorption was detectable in thermal programmed desorption (TPD) experiments. The Fig. 5.6 extrapolated value of $E_{a}=2.97$ $\mathrm{kJ} / \mathrm{mol}$ for dissociative chemisorption of octane seems in reasonable accord with the TPD experiments for which the octane desorption peak occurred at $277 \mathrm{~K}$ (c.f., $E_{a}=2.97 \mathrm{~kJ} / \mathrm{mol}$ with $\mathrm{RT}=2.3 \mathrm{~kJ} / \mathrm{mol}$ at $277 \mathrm{~K}$ ). Combined, PMMT theoretical calculations taken with thermal effusive beam and TPD experimental data indicate the activation energy for dissociative chemisorption of n-alkanes falls as the alkane size and depth of the physisorption well increases.

Figure 5.6 can be interpreted as an Evans-Polanyi plot under the assumption that the alkane $E_{D}$ serves as a good approximation to the van der Waals stabilization energy of the chemisorbed products of dissociative chemisorption given the $\mathrm{N}$-electron van der Waals interaction of the $\mathrm{RH}$ molecule should remain relatively constant through the dissociative chemisorption to yield $R_{(c)}+H_{(c)}$. Via a Haber cycle, the reaction exoergicity, $\Delta_{r} E$, for dissociative chemisorption of an n-alkane RH can be broken down as the energy required to break an R-H bond in the gas-phase minus the sum of a C-Pt(111) covalent bond energy, a H$\operatorname{Pt}(111)$ covalent bond energy, and the van der Waals stabilization energies for the chemisorbed $\mathrm{R}$ and $\mathrm{H}$ products on $\mathrm{Pt}(111)$. Assuming the van der Waals stabilization energy of the chemisorbed products is well approximated by $E_{D}$ for $\mathrm{RH}$, and the variation of the $\mathrm{R}-\mathrm{H}$ bond energy is negligible in comparison to the variation of $E_{D}$ with alkane size, then a reasonable approximation should be $\Delta_{r} E \approx \xi-E_{D}$ where $\xi$ is a constant. The Evans-Polanyi assumption is that $E_{a}$ should depend linearly on $\Delta_{r} E$ for a series of related reactions such that $E_{a}=\gamma \Delta_{r} E+\beta$. Substituting our approximate $\Delta_{r} E$ for the n-alkanes on $\operatorname{Pt}(111)$, yields $E_{a}=-\gamma E_{D}+(\gamma \xi+\beta)$ where the bracketed term is a constant. 
The Evans-Polanyi relation shown in Fig. 5.6 suggests that van der Waals interaction between the chemisorbed products of dissociative chemisorption and the surface may play a dominating role in determining the variation of the activation energy for dissociative chemisorption of the n-alkanes. Traditional GGA-DFT calculations of reaction energetics do not directly account for van der Waals interactions, ${ }^{35}$ and accordingly threshold energies for alkane dissociative chemisorption on metals calculated by DFT methods typically vary little with alkane size. ${ }^{20,21,36}$ Adding van der Waals corrections to DFT calculated reaction energetics is beginning to be explored in the theoretical literature of alkane reactivity at surfaces. ${ }^{37}$

\subsection{F. Gas-Surface Energy Exchange}

The non-equilibrium dissociative sticking coefficients $S_{n}\left(T_{g}, T_{s}\right)$ for ethane and propane on $\mathrm{Pt}(111)$ gave clear evidence for substantive gas-surface energy transfer. As discussed above, the net gas-surface energy transfer must sum to zero under thermal equilibrium conditions by detailed balance, and so the thermal dissociative sticking coefficient $S(T)$ is not influenced by specific rates of gas-surface energy transfer [c.f., s-PMMT ( $\alpha=0)$ and ME-PMMT ( $\alpha=1400$ $\mathrm{cm}^{-1}$ ) simulations of the propane $S(T)$ in Fig. 5.4]. As a close approximation to $S(T)$, the quasithermal dissociative sticking coefficients $S_{n}(T)$ measured in effusive beam experiments is relatively insensitive to changes in gas-surface energy transfer rates, particularly for larger molecules with many degrees of freedom. Alternatively, $S(T)$ can be derived experimentally through thermal bulb experiments or calculated based on integration of the $\cos (\vartheta) / \pi$ thermal molecular flux over the $S(T, \vartheta)$ that can be measured in effusive molecular beam experiments. Ultimately, the experimental hallmark of gas-surface energy transfer is the tendency of the non- 
equilibrium dissociative sticking coefficients $S_{n}\left(T_{g}, T_{s}\right)$ to collapse towards the $S(T)$ curve (or its $S_{n}(T)$ surrogate).

The gas-surface energy transfer parameters of $\alpha=50 \mathrm{~cm}^{-1}, 350 \mathrm{~cm}^{-1}$, and $1400 \mathrm{~cm}^{-1}$ for methane, ethane, and propane on $\operatorname{Pt}(111)$ are believed to be amongst the first to be determined at reactive energies for molecules interacting with a clean metal surface for the Eq. 5.7 kind of exponential energy down PC/substrate energy transfer model that is commonly employed in studies of gas-phase energy transfer and reactivity. ${ }^{38-40}$

Finally, it is worth reconsidering the Fig. 5.6 Evans-Polanyi plot based on the premise that $E_{a}=\gamma \Delta_{r} E+\beta \approx-\gamma E_{D}+E_{a, 0}$. The slope of Fig. 5.6 yields $\gamma=0.60$ which is indicative of a transition state that is slightly late for dissociative chemisorption. DFT calculations, which overestimate the experimentally determined threshold energies for alkane dissociative chemisorption on metals [c.f. Fig. 3.23(a)], typically find late transition states for dissociative chemisorption such that $\gamma \sim 0.9$ for $\Delta_{r} E$ changes. ${ }^{2,41}$ In contrast, Beck and co-workers ${ }^{42}$ have experimentally shown that the vibrational efficacy for dissociative chemisorption of $\mathrm{CH}_{4}$ on $\mathrm{Pt}(111)$ for 2 quanta of $\mathrm{C}-\mathrm{H}$ asymmetric stretch is $\eta_{v}\left(2 v_{3}\right)=0.38$, and d-PMMT modeling indicates a thermally averaged vibrational efficacy of 0.40 for this system. These $\eta_{v}$ values suggest the $\mathrm{CH}_{4} / \mathrm{Pt}(111)$ transition state barrier is relatively early with respect to $\mathrm{C}-\mathrm{H}$ bond extension according to the Polanyi rules ${ }^{43}$ for direct reactions, but the Evans-Polanyi plot shown in Fig. 5.6 suggests the transition state is somewhat late with respect to van der Waals bond formation. State-selective reactivity in polyatomic molecules occurring on a multi-dimensional potential energy surface, even in the gas-phase, ${ }^{44}$ is not as well understood as the lower dimensionality atom + diatomic molecule reactivity for which the Polanyi rules were developed. A larger vibrational efficacy, $\eta_{v} \gtrsim 1$, for promoting reactivity would be anticipated for a late 
transition state ${ }^{19}$. It seems that the multi-dimensional nature of the reactive potential energy surface for polyatomic molecule/surface reaction makes absolute descriptions of "early" and "late" transition state characteristics somewhat problematic.

\subsection{G. Time Dependent Reactivity}

Figure 5.8 shows the time evolution over 40 ps of the precursor complex coverage distributions for a momentary incident flux of molecules at $\mathrm{T}_{\mathrm{g}}=700 \mathrm{~K}$ striking an isothermal surface along the direction of the surface normal . The ME-PMMT parameters for this simulation were based on the s-PMMT model for $\mathrm{CH}_{4} / \mathrm{Pt}(111)$ given in Chapter 3 $\left\{E_{0}=47.3 \mathrm{~kJ} / \mathrm{mol}, s=3, \alpha=50 \mathrm{~cm}^{-1}\right\}$ without tunneling and with active rotations. The precursor complex coverage distributions are renormalized to one for each time, t. The inset describes the PC coverage variation over time. Within the first picosecond, $90 \%$ of the initial PCs have either reacted or desorbed, and by two picoseconds only $1 \%$ of the initial distribution remains. From the initial distribution at $\mathrm{t}=0$, only a small fraction of $\mathrm{PC}$ 's from the initial collision, $S_{n}\left(T=700 K, \vartheta=0^{\circ}\right)=4.6^{*} 10^{-4}$, surmount the barrier and successfully react. At long surfaces lifetimes, the remaining precursor complexes have a distribution that is substantially hypothermal, $\mathrm{T}_{\text {eff }}=353 \mathrm{~K}$, because the high energy tail of a $700 \mathrm{~K}$ thermal Boltzmann distribution cannot be regenerated fast enough by energy transfer due to rapid desorption. Even at thermal equilibrium, a thermal rate constant treatment of activated dissociative chemisorption kinetics is problematic because the steady state coverage distributions can be intrinsically non-equilibrium in the face of rapid desorption. Better is to use a master equation microcanonical treatment of the reaction kinetics.

\subsection{Summary}


A master equation precursor-mediated microcanonical trapping (ME-PMMT) model of activated dissociative chemisorption was used to simulate thermal effusive beam experiments and extract transition state and gas-surface energy transfer parameters from the experimental data. Threshold energies for dissociative chemisorption of methane, ethane, and propane on $\operatorname{Pt}(111)$ were $E_{0}=58.9 \mathrm{~kJ} / \mathrm{mol}, 34.4 \mathrm{~kJ} / \mathrm{mol}$, and $23.6 \mathrm{~kJ} / \mathrm{mol}$ respectively. Exponential down gas-surface energy transfer parameters of $\alpha=50 \mathrm{~cm}^{-1}, 700 \mathrm{~cm}^{-1}$, and $1400 \mathrm{~cm}^{-1}$ for methane, ethane, and propane on $\mathrm{Pt}(111)$ were determined. d-PMMT threshold energies for alkane dissociative chemisorption were consistently lower than those values calculated from DFT potential energy surfaces. The linear correlation between the experimental activation energy for alkane quasi-thermal dissociative sticking and the alkane desorption energy from the physisorption well, $E_{a}=\gamma \Delta_{r} E+\beta \approx-\gamma E_{D}+E_{a, 0}$, was interpreted as an Evans-Polanyi relation because the alkane desorption energy should approximate the van der Waals stabilization energy of the chemisorbed products of alkane dissociative chemisorption. The $\gamma=$ 0.60 slope of the Evans-Polanyi plot suggests a transition state for alkane dissociative chemisorption that is slightly late with respect to van der Waals stabilization of the chemisorbed products. The Evans-Polanyi correlation of $E_{a}$ with $E_{D}$ is strongly suggestive that a full treatment of van der Waals interactions will be essential to achieve chemical accuracy in electronic structure theory calculations of reaction energetics for alkane reactions at surfaces. ${ }^{37}$ 

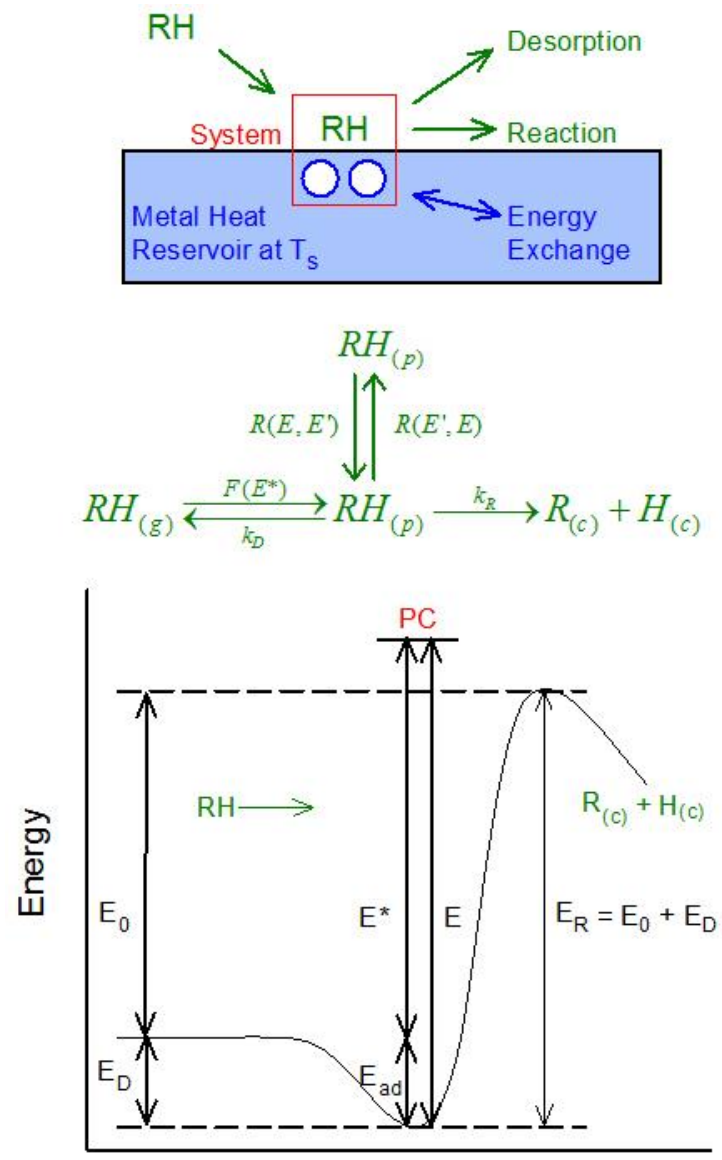

Reaction Coordinate

Figure 5.1. Schematic depiction of the kinetics and energetics of activated dissociative chemisorption for an alkane, RH. Microcanonical trapping is posited to occur for the collisionally formed precursor complex, PC, comprised of an alkane interacting with $s$ surface oscillators in the spatial vicinity of the physisorption well minimum. Zero-point energies are implicitly included within the potential energy curve along the reaction coordinate. Refer to the text for further details. 


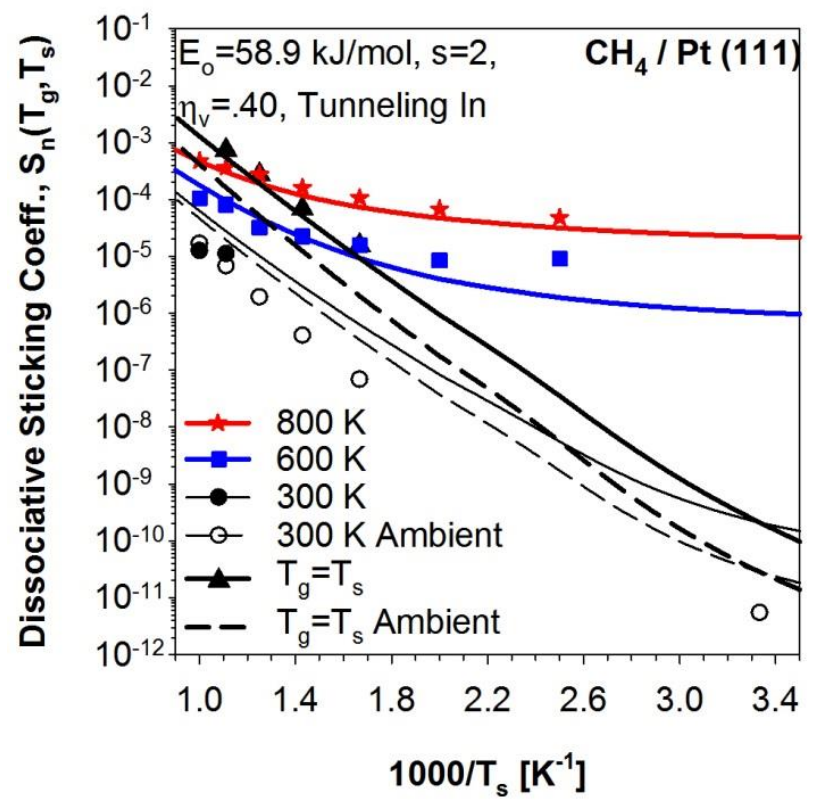

Figure 5.2. d-PMMT simulations (lines) of $S_{n}\left(T_{g}, T_{s}\right)$ for methane on $\operatorname{Pt}(111)$ are compared to experiments(points) for a normally incident thermal gas from an effusive source. The d-PMMT simulations are based on a parameter set, $\left\{E_{0}=58.9 \mathrm{~kJ} / \mathrm{mol}, s=2, \eta_{v}=0.40\right\},{ }^{45454545454545}$ that quantitatively replicates a diverse range of dissociative sticking behavior for $\mathrm{CH}_{4} / \mathrm{Pt}(111){ }^{46,47}$ 

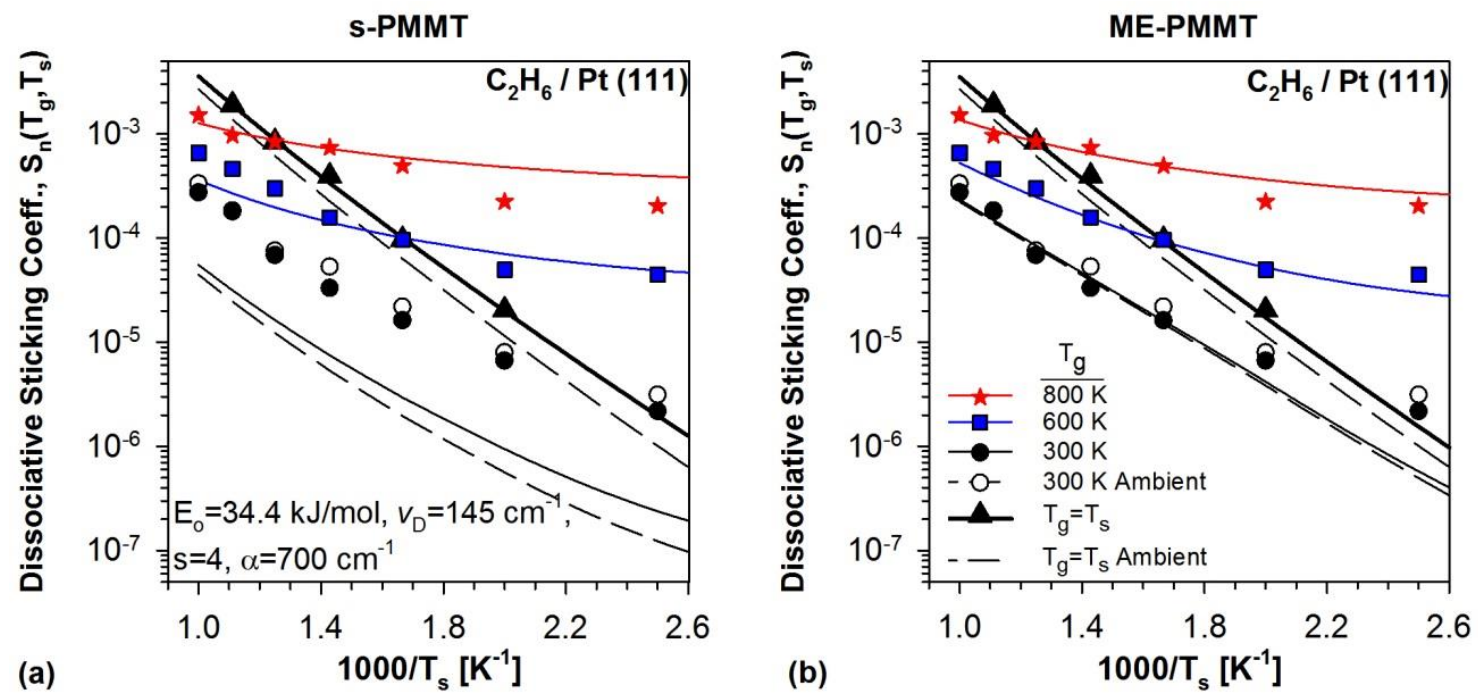

Figure 5.3. (a) s-PMMT and (b) ME-PMMT simulations (lines) of $S_{n}\left(T_{g}, T_{s}\right)$ for ethane on $\operatorname{Pt}(111)$ using the parameter set: $\left\{E_{0}=34.4 \mathrm{~kJ} / \mathrm{mol}, \mathrm{s}=4, v_{D}=145 \mathrm{~cm}^{-1}, \alpha=700 \mathrm{~cm}^{-1}\right\}$. The $\alpha$ parameter was used only for the ME-PMMT simulations and is roughly equivalent to the average energy transfered in an energy downward collision. ${ }^{25}$ 

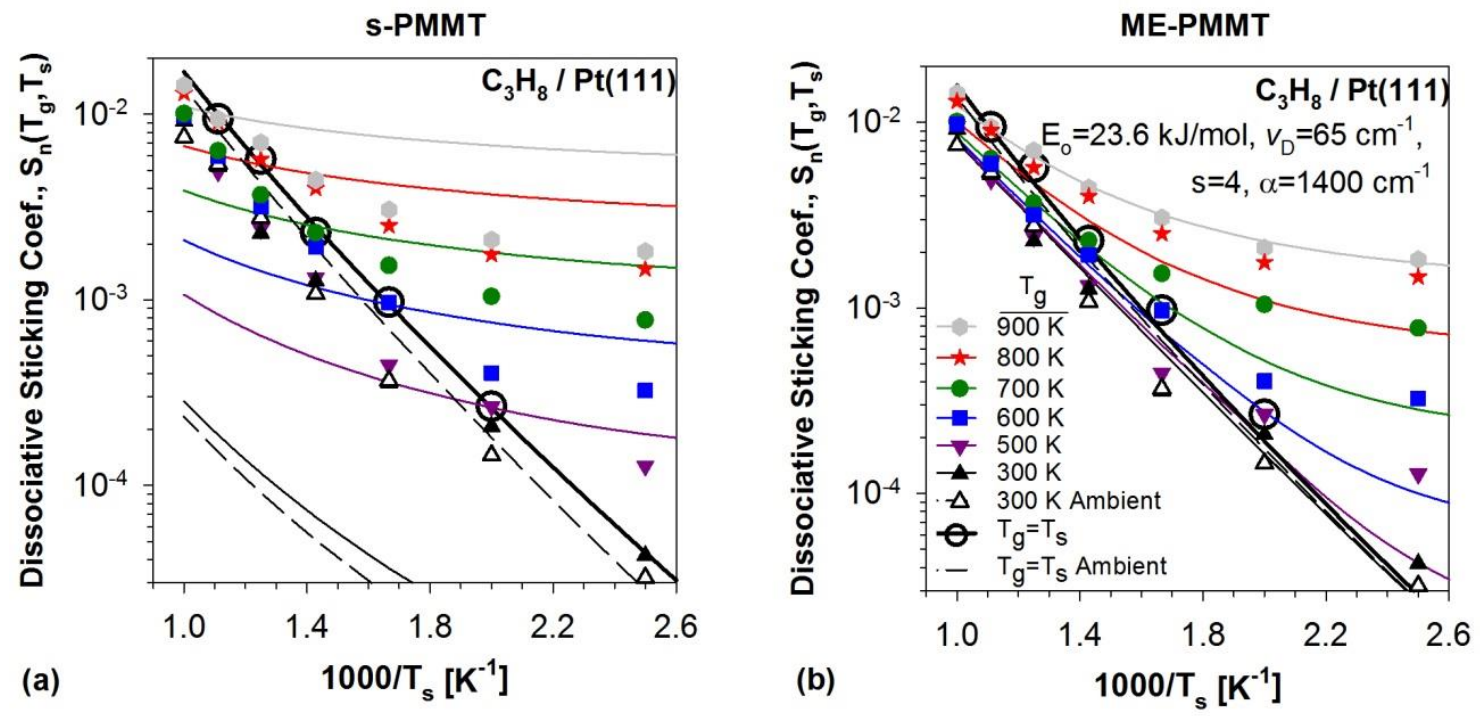

Figure 5.4. (a) s-PMMT and (b) ME-PMMT simulations (lines) of $S_{n}\left(T_{g}, T_{s}\right)$ for propane on $\operatorname{Pt}(111)$ using the parameter set: $\left\{E_{0}=23.6 \mathrm{~kJ} / \mathrm{mol}, s=4, v_{D}=65 \mathrm{~cm}^{-1}, \alpha=1400 \mathrm{~cm}^{-1}\right\}$. The $\alpha$ parameter was used only for the ME-PMMT simulations and is roughly equivalent to the average energy transfered in an energy downward collision. ${ }^{25}$ 

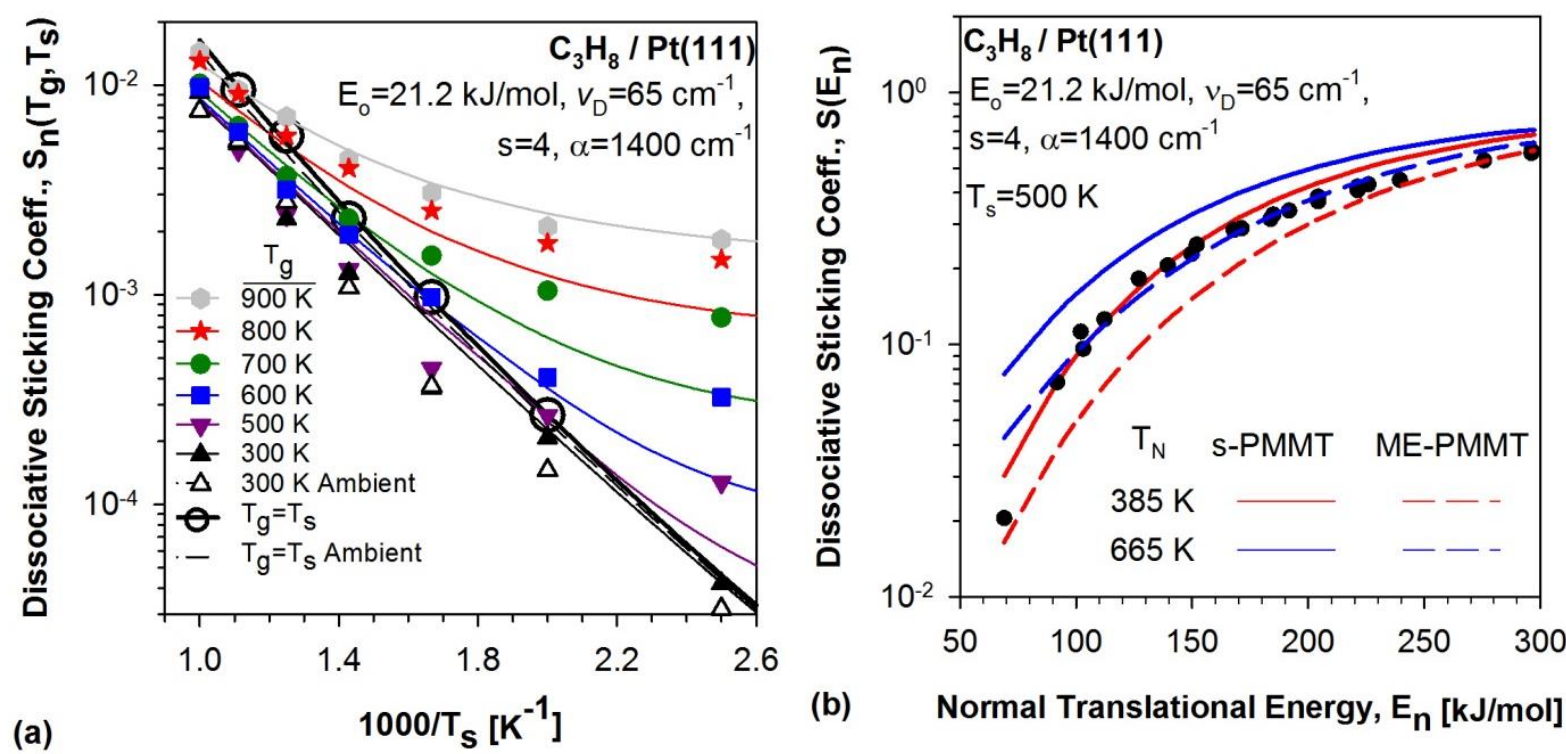

(b) Normal Translational Energy, $E_{n}[\mathrm{~kJ} / \mathrm{mol}]$

Figure 5.5. ME-PMMT simulations (lines) of (a) $S_{n}\left(T_{g}, T_{s}\right)^{25}$ and (b) $S\left(E_{n}, T_{N}\right)^{31}$ for propane on $\operatorname{Pt}(111)$ are compared to measurements (points). 


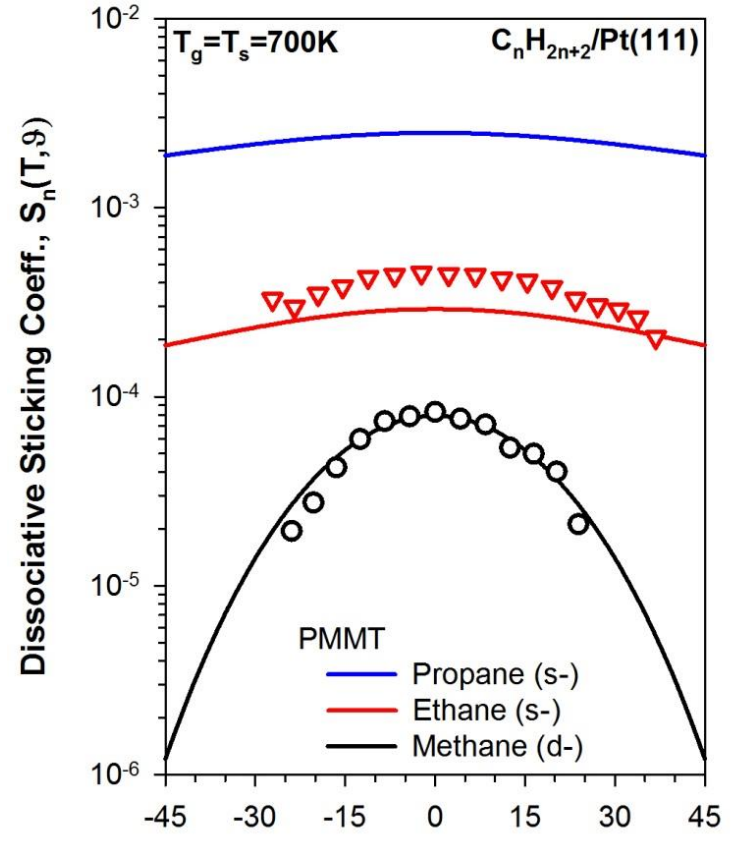

(a)

Angle, $\vartheta$ [Degrees]

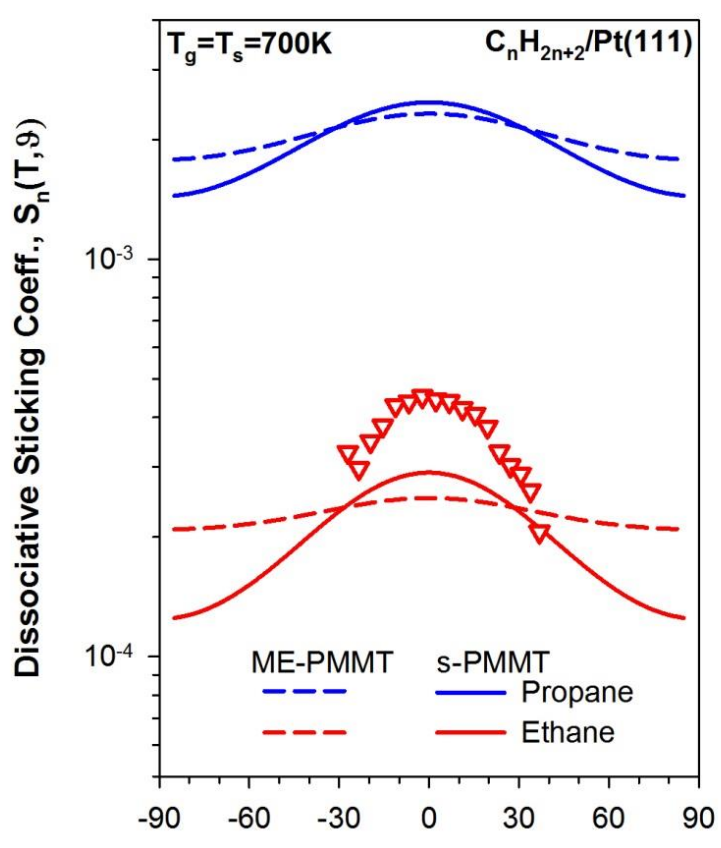

(b)

Angle, $\vartheta$ [Degrees]

Fig. 5.6. Light alkane/Pt(111) angle-resolved thermal dissociative sticking coefficient, $S(T=700 K, \vartheta)$, calculated using for (a) s-PMMT (ethane and propane) or d-PMMT (methane) and (b) ME-PMMT models (lines) are compared to experiments (open points). 


\begin{tabular}{lllll}
\hline Alkane & PMMT & A & B & n \\
\hline Methane & s- & $1 * 10^{-21}$ & $5.0^{* 10^{-5}}$ & 12.1 \\
Ethane & s- & $1.23 * 10^{-4}$ & $1.68 * 10^{-4}$ & 2.8 \\
& ME- & $2.09 * 10^{-4}$ & $0.42 * 10^{-4}$ & 2.4 \\
& & & & \\
Propane & s- & $1.42 * 10^{-3}$ & $1.06 * 10^{-3}$ & 2.2 \\
& ME- & $1.78 * 10^{-3}$ & $0.54 * 10^{-3}$ & 2 \\
\hline
\end{tabular}

Table 5.1. Comparison of fits, $S(T, \vartheta)=A+B \cos ^{n}(\vartheta)$, to s- and ME-PMMT calculated dissociative sticking coefficients of light alkanes on $\mathrm{Pt}(111)$ at $\mathrm{T}=700 \mathrm{~K}$. 


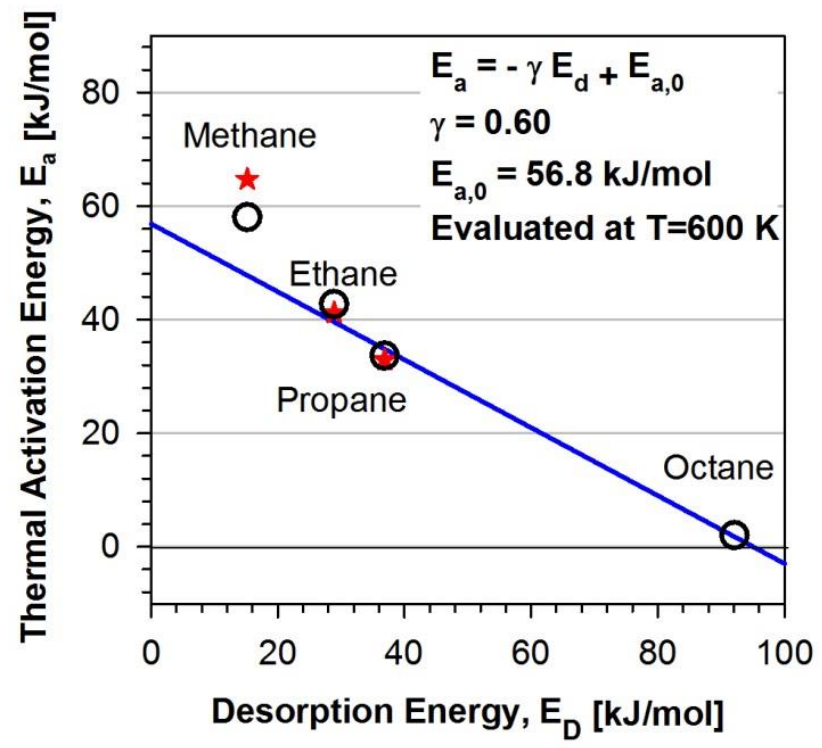

Figure 5.7. Evans-Polanyi plot of the $S_{n}(T)$-derived activation energy for dissociative chemisorption versus desorption energy ${ }^{30}$ for several alkanes on $\operatorname{Pt}(111)$. Red stars denote PMMT calculated activation energies at $\mathrm{T}=600 \mathrm{~K}$ while open circles are experimentally determined activation energies ${ }^{25}$. 


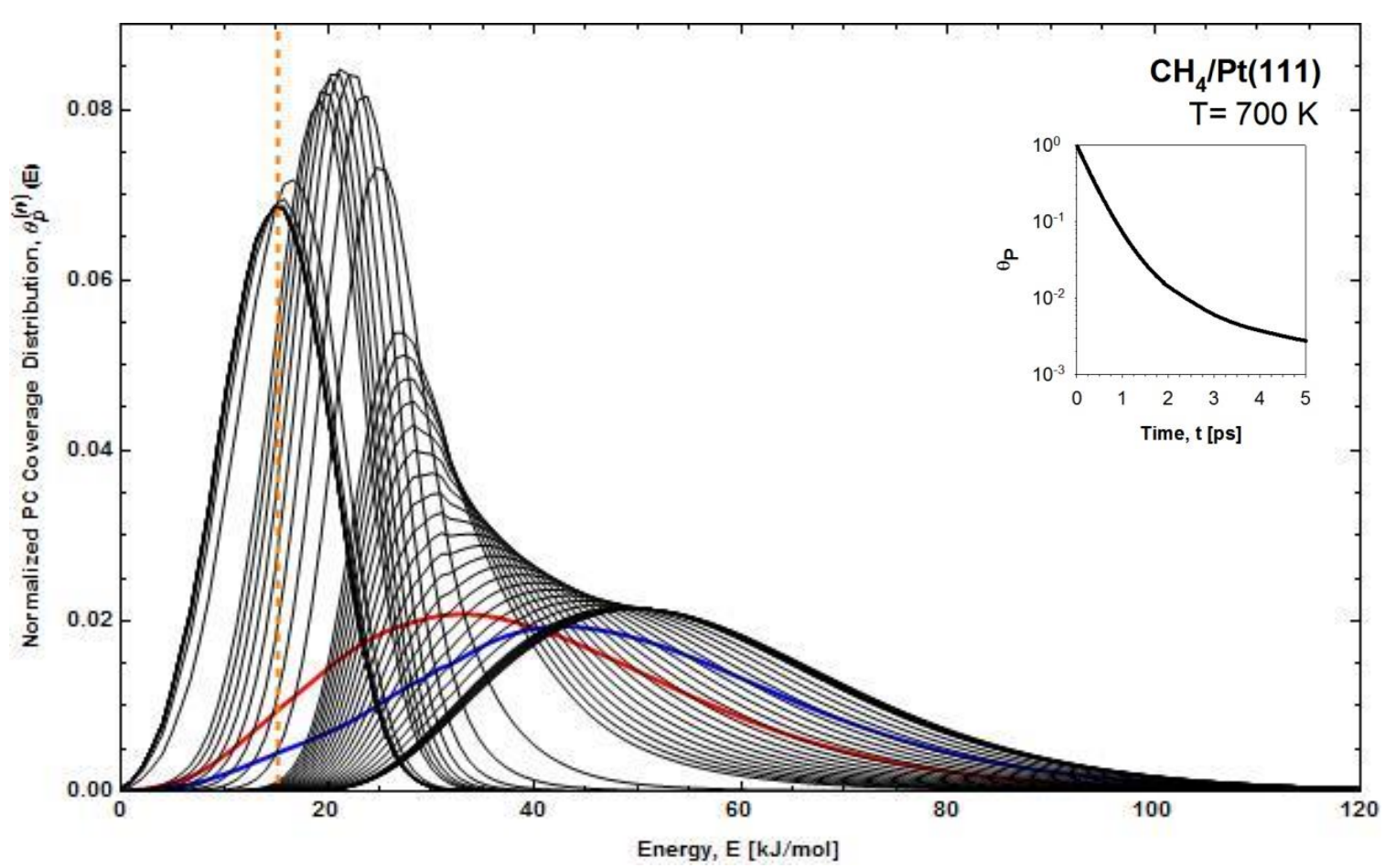

Figure 5.8. ME-PMMT precursor complex coverage distributions (black) are shown from $t=0$ to 40ps, along with a Boltzmann distribution (red) and steady state coverage distribution (blue). The energy scale, E, has its zero at the zero-point level of the physisorption well and the vacuum level at $+\mathrm{E}_{\mathrm{d}}, 15.7 \mathrm{~kJ} / \mathrm{mol}$, which is marked by a vertical, dashed, orange line. (inset) ME-PMMT predicted precursor complex coverage as a function of surface lifetime with parameter set $\left\{E_{0}=47.3 \mathrm{~kJ} / \mathrm{mol}, s=3, \alpha=50 \mathrm{~cm}^{-1}\right\}$ without tunneling but with active rotations. Initial conditions at $\mathrm{t}=0$ are for a normally incident puff of gas at $\mathrm{T}_{\mathrm{g}}=700 \mathrm{~K}$ on to a clean $\operatorname{Pt}(111)$ surface. 


\section{References:}

1. Labinger, J. A.; Bercaw, J. E., Understanding and exploiting C-H bond activation. Nature 2002, 417, (6888), 507-514.

2. $\quad$ Bligaard, T.; Norskov, J. K.; Dahl, S.; Matthiesen, J.; Christensen, C. H.; Sehested, J., The Bronsted-Evans-Polanyi relation and the volcano curve in heterogeneous catalysis. J. Catal. 2004, 224, (1), 206-217.

3. Weaver, J. F.; Carlsson, A. F.; Madix, R. J., The adsorption and reaction of low molecular weight alkanes on metallic single crystal surfaces. Surf. Sci. Rep. 2003, 50, (4-5), $107-$ 199.

4. Wei, J. M.; Iglesia, E., Isotopic and kinetic assessment of the mechanism of reactions of $\mathrm{CH}_{4}$ with $\mathrm{CO}_{2}$ or $\mathrm{H}_{2} \mathrm{O}$ to form synthesis gas and carbon on nickel catalysts. J. Catal. 2004, 224, (2), 370-383.

5. Wei, J. M.; Iglesia, E., Mechanism and site requirements for activation and chemical conversion of methane on supported Pt clusters and turnover rate comparisons among noble metals. J. Phys. Chem. B 2004, 108, (13), 4094-4103.

6. Nielsen, B. O.; Luntz, A. C.; Holmblad, P. M.; Chorkendorff, I., Activated Dissociative Chemisorption of Methane on Ni(100) - a Direct Mechanism under Thermal Conditions. Catal. Lett. 1995, 32, (1-2), 15-30.

7. Abbott, H. L.; Harrison, I., Methane dissociative chemisorption on $\mathrm{Ru}(0001)$ and comparison to metal nanocatalysts. J. Catal. 2008, 254, (1), 27-38.

8. Wu, M.-C.; Goodman, D. W., High-resolution electron energy-loss measurements of sticking coefficients of methane decomposition on Ru(0001). Surf. Sci. 1994, 306, (1-2), L529.

9. Beebe, T. P.; Goodman, D. W.; Kay, B. D.; Yates, J. T., Kinetics of the Activated Dissociative Adsorption of Methane on the Low Index Planes of Nickel Single-Crystal Surfaces. J. Chem. Phys. 1987, 87, (4), 2305-2315.

10. Sault, A. G.; Goodman, D. W., Dissociative adsorption of alkanes on Ni(100) comparison with molecular-beam results. J. Chem. Phys. 1988, 88, (11), 7232-7239.

11. Rettner, C. T.; Pfnur, H. E.; Auerbach, D. J., Dissociative Chemisorption of CH4 on W(110) - Dramatic Activation by Initial Kinetic-Energy. Phys. Rev. Lett. 1985, 54, (25), 27162719.

12. Lee, M. B.; Yang, Q. Y.; Tang, S. L.; Ceyer, S. T., Activated Dissociative Chemisorption of $\mathrm{CH} 4$ on Ni(111) - Observation of a Methyl Radical and Implication for the Pressure Gap in Catalysis. J. Chem. Phys. 1986, 85, (3), 1693-1694.

13. Schoofs, G. R.; Arumainayagam, C. R.; Mcmaster, M. C.; Madix, R. J., Dissociative Chemisorption of Methane on Pt(111). Surf. Sci. 1989, 215, (1-2), 1-28.

14. Holmblad, P. M.; Wambach, J.; Chorkendorff, I., Molecular-Beam Study of Dissociative Sticking of Methane on Ni(100). J. Chem. Phys. 1995, 102, (20), 8255-8263.

15. Juurlink, L. B. F.; McCabe, P. R.; Smith, R. R.; DiCologero, C. L.; Utz, A. L., Eigenstate-resolved studies of gas-surface reactivity: $\mathrm{CH}_{4}\left(\mathrm{n}_{3}\right)$ dissociation on $\mathrm{Ni}(100)$. Phys. Rev. Lett. 1999, 83, (4), 868-871.

16. Higgins, J.; Conjusteau, A.; Scoles, G.; Bernasek, S. L., State selective vibrational $\left(2 \mathrm{n}_{3}\right)$ activation of the chemisorption of methane on Pt (111). J. Chem. Phys. 2001, 114, (12), 52775283 .

17. Beck, R. D.; Maroni, P.; Papageorgopoulos, D. C.; Dang, T. T.; Schmid, M. P.; Rizzo, T. R., Vibrational mode-specific reaction of methane on a nickel surface. Science 2003, 302, (5642), 98-100.

18. Bisson, R.; Dang, T. T.; Sacchi, M.; Beck, R. D., Vibrational activation in direct and precursor-mediated chemisorption of SiH4 on Si(100). J. Chem. Phys. 2008, 129, (8), 081103. 
19. Juurlink, L. B. F.; Killelea, D. R.; Utz, A. L., State-resolved probes of methane dissociation dynamics. Prog. Surf. Sci. 2009, 84, (3-4), 69-134.

20. Chen, Y.; Vlachos, D. G., Hydrogenation of Ethylene and Dehydrogenation and Hydrogenolysis of Ethane on Pt(111) and Pt(211): A Density Functional Theory Study. J. Phys. Chem. C 114, (11), 4973-4982.

21. Anghel, A. T.; Wales, D. J.; Jenkins, S. J.; King, D. A., Pathways for dissociative ethane chemisorption on Pt $\{110\}$ (1x2) using density functional theory. Chem. Phys. Lett. 2005, 413, (46), 289-293.

22. DeWitt, K. M.; Valadez, L.; Abbott, H. L.; Kolasinski, K. W.; Harrison, I., Effusive molecular beam study of $\mathrm{C}_{2} \mathrm{H}_{6}$ dissociation on $\mathrm{Pt}(111)$. J. Phys. Chem. B 2006, 110, (13), 67146720 .

23. Rodriguez, J. A.; Goodman, D. W., Dissociative Adsorption And Hydrogenolysis of Ethane Over Clean And Ni-Covered Pt(111). J. Phys. Chem. 1990, 94, (13), 5342-5347.

24. Cushing, G. W.; Navin, J. K.; Donald, S. B.; Valadez, L.; Johánek, V.; Harrison, I., Addition/Correction to: C-H Bond Activation of Light Alkanes on Pt(111): Dissociative Sticking Coefficients, Evans-Polanyi Relation, and Gas-Surface Energy Transfer (vol 114, pg 17222, 2010). J. Phys. Chem. C 2010, 114, (51), 22790-22790.

25. Cushing, G. W.; Navin, J. K.; Donald, S. B.; Valadez, L.; Johanek, V.; Harrison, I., C-H Bond Activation of Light Alkanes on Pt(111): Dissociative Sticking Coefficients, Evans-Polanyi Relation, and Gas-Surface Energy Transfer. J. Phys. Chem. C 2010, 114, (40), 17222-17232.

26. Vajda, S.; Pellin, M. J.; Greeley, J. P.; Marshall, C. L.; Curtiss, L. A.; Ballentine, G. A.; Elam, J. W.; Catillon-Mucherie, S.; Redfern, P. C.; Mehmood, F.; Zapol, P., Subnanometre platinum clusters as highly active and selective catalysts for the oxidative dehydrogenation of propane. Nature Materials 2009, 8, (3), 213-216.

27. Barker, J. R.; Yoder, L. M.; King, K. D., Vibrational energy transfer modeling of nonequilibrium polyatomic reaction systems. J. Phys. Chem. A 2001, 105, (5), 796-809.

28. Bukoski, A.; Blumling, D.; Harrison, I., Microcanonical unimolecular rate theory at surfaces. I. Dissociative chemisorption of methane on Pt(111). J. Chem. Phys. 2003, 118, (2), 843-871.

29. Bukoski, A.; Abbott, H. L.; Harrison, I., Microcanonical Unimolecular Rate Theory at Surfaces. III. Thermal Dissociative Chemisorption of Methane on $\mathrm{Pt}(111)$ and Detailed Balance. Journal of Chemical Physics 2005, to be submitted January.

30. Tait, S. L.; Dohnalek, Z.; Campbell, C. T.; Kay, B. D., n-alkanes on Pt(111) and on $\mathrm{C}(0001) / \mathrm{Pt}(111)$ : Chain length dependence of kinetic desorption parameters. J. Chem. Phys. 2006, 125, (23), 234308.

31. Weaver, J. F.; Krzyzowski, M. A.; Madix, R. J., Direct dissociative chemisorption of alkanes on Pt(111): Influence of molecular complexity. J. Chem. Phys. 2000, 112, (1), 396-407. 32. Scoles (Ed.), G., Atomic and Molecular Beam Methods. Oxford Univ. Press: New York, 1988; Vol. 1.

33. Rettner, C. T.; Pfnur, H. E.; Auerbach, D. J., On the Role of Vibrational-Energy in the Activated Dissociative Chemisorption of Methane on Tungsten and Rhodium. J. Chem. Phys. 1986, 84, (8), 4163-4167.

34. Graham, A. P., The low energy dynamics of adsorbates on metal surfaces investigated with helium atom scattering. Surf. Sci. Rep. 2003, 49, (4-5), 115-168.

35. Cramer, C. J.; Truhlar, D. G., Density functional theory for transition metals and transition metal chemistry. Phys. Chem. Chem. Phys. 2009, 11, (46), 10757-10816.

36. Anghel, A. T.; Wales, D. J.; Jenkins, S. J.; King, D. A., Pathways for dissociative methane chemisorption on $\operatorname{Pt}\{110\}-(1 \mathrm{x} 2)$. Phys. Rev. B 2005, 71, (11), 113410.

37. Rozanska, X.; Sauer, J., Oxidative conversion of C1-C3 alkanes by vanadium oxide catalysts. DFT results and their accuracy. Int. J. Quantum Chem 2008, 108, (12), 2223-2229. 
38. Oref, I.; Tardy, D. C., Energy-Transfer In Highly Excited Large Polyatomic-Molecules. Chem. Rev. 1990, 90, (8), 1407-1445.

39. Holbrook, K. A.; Pilling, M. J.; Robertson, S. H., Unimolecular Reactions. Wiley: Chichester, UK, 1996.

40. Forst, W., Unimolecular Reactions: A Concise Introduction. 1st ed.; Cambridge University Press: Cambridge UK, 2003.

41. Santen, R. A. v.; Neurock, M.; Shetty, S. G., Reactivity Theory of Transition-Metal Surfaces: A Bronsted-Evans-Polanyi Linear Activation Energy-Free-Energy Analysis. Chem. Rev. 2009, 110, (4), 2005-2048.

42. Bisson, R.; Sacchi, M.; Dang, T. T.; Yoder, B.; Maroni, P.; Beck, R. D., State-resolved reactivity of $\mathrm{CH}_{4}\left(2 \mathrm{n}_{3}\right)$ on $\mathrm{Pt}(111)$ and $\mathrm{Ni}(111)$ : Effects of barrier height and transition state location. J. Phys. Chem. A 2007, 111, (49), 12679-12683.

43. Polanyi, J. C.; Wong, W. H., Location of Energy Barriers. I. Effect on the Dynamics of Reactions A + BC. J. Chem. Phys. 1969, 51, 1439-1450.

44. Zhang, W. Q.; Kawamata, H.; Liu, K. P., CH Stretching Excitation in the Early Barrier F $+\mathrm{CHD}_{3}$ Reaction Inhibits CH Bond Cleavage. Science 2009, 325, (5938), 303-306.

45. DeWitt, K. M.; Valadez, L.; Abbott, H. L.; Kolasinski, K. W.; Harrison, I., Using effusive molecular beams and microcanonical unimolecular rate theory to characterize $\mathrm{CH}_{4}$ dissociation on Pt(111). J. Phys. Chem. B 2006, 110, (13), 6705-6713.

46. Donald, S. B.; Harrison, I., Dynamically biased RRKM model of activated gas-surface reactivity: vibrational efficacy and rotation as a spectator in the dissociative chemisorption of $\mathrm{CH}_{4}$ on Pt(111). Phys. Chem. Chem. Phys. 2012, 14, (5), 1784-1796.

47. Donald, S. B., Thesis - Chapter 3. 2013. 